\section{R 676}

Technical Report

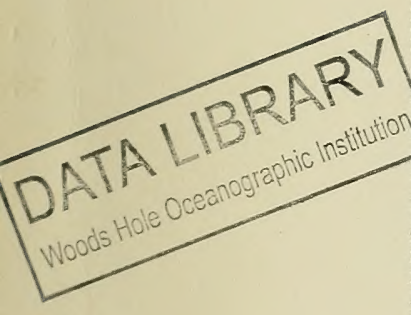

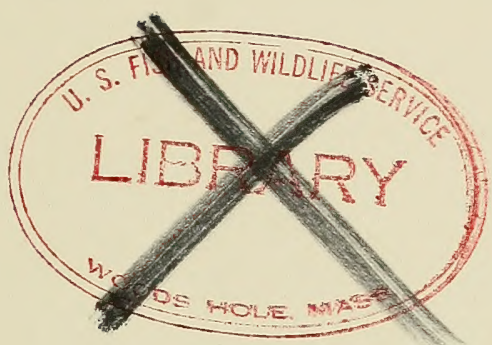

DEVELOPMENT OF A SPHERICAL

ACRYLIC PLASTIC PRESSURE HULL

FOR HYDROSPACE APPLICATION

MAY 1519 政

Sponsored by

NAVAL FACILITIES ENGINEERING COMMAND

NAVAL CIVIL ENGINEERING LABORATORY

Port Hueneme, California

This document has been approved for public release and sale; its distribution is unlimited. 


\title{
DEVELOPMENT OF A SPHERICAL ACRYLIC PLASTIC PRESSURE HULL FOR HYDROSPACE APPLICATION
}

\author{
Technical Report R-676 \\ YF 38.535.005.01.006 \\ by \\ J. D. Stachiw
}

\begin{abstract}
A spherical, acrylic plastic capsule has been designed for protection of man against the external hydrostatic pressure present at continental shelf depths. Experimental and analytical studies have been conducted to evaluate the performance of both the spherical capsule design and the acrylic plastic construction material at continental shelf depths. Results from testing twenty-two 15-inch-outside diameter models and a large-scale prototype under short-term, cyclic, and long-term hydrostatic pressure indicate that the design and material chosen meet the requirements for safe operation at continental shelf depths. A prototype 66-inch-OD capsule of 2.5-inch wall thickness, and 4,000-pound positive buoyancy in seawater has been specifically developed for the NEMO (Naval Experimental Manned Observatory) system. The NEMO prototype capsule successfully withstood 105 simulated dives ranging from 250 to 2,400 feet prior to being tested to implosion at a simulated depth of 4,150 feet. The experimental data indicate that the fullscale NEMO capsule can be safely subjected to brief proof-test dives to 1,200 feet and routine operational manned dives of extended duration to 600 feet.
\end{abstract}

This document has been approved for public release and sale; its distribution is unlimited.

Copies available at the Clearinghouse for Federal Scientific and Technical Information (CFSTI), Sills Building, 5285 Port Royal Road, Springfield, Va. 22151 


\section{CONTENTS}

page

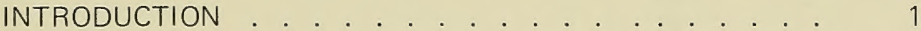

Statement of the Problem . . . . . . . . . 1

Objective of Study . . . . . . . . . . . . . . 2

Background Information . . . . . . . . . . . 2

Scope of Investigation . . . . . . . . . . . . . 7

PROGRAM PLAN . . . . . . . . . . . . . . . 8

PHASE 1-DESIGN OF NEMO CAPSULE . . . . . . . . . 9

General Design. . . . . . . . . . . . . . . 9

Detail Design . . . . . . . . . . . . . . 11

PHASE 2-FABRICATION PROCESS.......... . . 38

Acrylic Hull Fabrication . . . . . . . . . . . 38

Polar Inserts . . . . . . . . . . . . . . 86

PHASES 3 AND 4-EVALUATION OF DESIGN AND

FABRICATION . . . . . . . . . . . . . . 87

Capsule Test Specimens . . . . . . . . . . . 87

Testing Program for Capsules . . . . . . . . . . . 90

Instrumentation . . . . . . . . . . . 109

TEST FINDINGS . . . . . . . . . . . . . . 117

Model Capsules . . . . . . . . . . . . 117

Large-Scale Capsule . . . . . . . . . . . . . 135

SUMMARY OF FINDINGS . . . . . . . . . . 155

CONCLUSION . . . . . . . . . . . . . 156

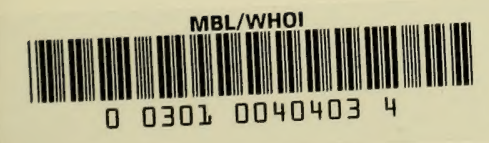


APPENDIXES

A - Detailed Dimensions of 66-Inch-Diameter Acrylic Plastic Hull for NEMO System . . . . . . . . . . 160

B - Photoelastic Investigation of Capsules . . . . . . . 175

C - Dimensional Deviations of NEMO Models . . . . . . 183

D - Feasibility Study of Equatorial Ring for the Attachment of the Acrylic Plastic Capsule to the Framework of a Submersible System . . . . . . . 187

E - Exploratory Investigation into the Feasibility of Acrylic Plastic Capsules for Ocean Depths Beyond 600 Feet . . . . . . . . . . . . . . 195

F - Fabrication of Operational 66-Inch-OD x 61-Inch-ID Acrylic Capsules . . . . . . . . . . 202

REFERENCES . . . . . . . . . . . 215 


\section{INTRODUCTION}

\section{Statement of the Problem}

Performance of useful tasks by man in hydrospace or on the ocean floor safely and efficiently requires good viewing and visibility. Ideally, underwater workers should retain stereoscopic, polychromatic vision in all directions. Furthermore, to optimize physical and mental efforts, it is desirable that the working environment be the gaseous mixture and pressure commonly referred to as "earth atmosphere." Since man immersed directly in water retains neither his terrestrial vision nor atmosphere, it is necessary to provide a protective capsule or habitat.

Pressure-resistant, transparent capsules can enclose man in a 1-atmosphere environment and at the same time not limit his vision in any manner. There are transparent structural materials such as plastics and glass for fabricating such pressure-resistant capsules. The major obstacles to their use are (1) the lack of data on the behavior of transparent materials under the biaxial and triaxial compressive stresses generally found in a pressure hull under cyclic or long-term external hydrostatic pressure, (2) the lack of proven designs for transparent capsules in which the penetrations in the form of hatches and feedthroughs have been incorporated, and (3) the lack of economical methods for fabricating transparent materials that result in reliable pressure-resistant capsules.

Because proven transparent capsule designs, methods of building them economically, and data on behavior of transparent material in such capsules under hydrostatic pressure have not been available, no transparent capsules have been built and used in hydrospace to date. If a pressureresistant capsule incorporating hatches and feedthroughs could be designed to utilize transparent material for its pressure hull, and if such a capsule could be built from a transparent material that safely withstands the stresses generated by hydrostatic pressure, transparent capsules would replace in many cases the steel capsules with small portholes currently utilized in submersibles. 


\section{Objective of Study}

The objective of this study was to explore analytically and experimentally the feasibility of constructing a 120-inch man-rated, pressure-resistant, transparent capsule for continental shelf operation with a minimum life of 2 years and 100 dives to its operational depth.

The capsule design was to be of general nature so that a scaled-down version could be incorporated into many available or foreseeable hardware systems designed for operation at continental shelf depths.

The data generated in this exploratory study were to serve two purposes: (1) they were to permit the cognizant certification authorities to assess the seaworthiness of the 66-inch-diameter* capsules built during this study for the NEMO system, and (2) they were to serve as a basis for design of other pressure-resistant, transparent capsules of the same material, but different configuration or dimensions.

\section{Background Information}

The idea that a pressure-resistant, transparent capsule is technically feasible and that its use will make exploration of hydrospace more comprehensive and effective is not new. It was suggested by Professor Piccard ${ }^{1}$ in 1956 that such a capsule could replace steel pressure hulls. He proposed that a spherical capsule be assembled by bonding 12 spherical pentagons cast from acrylic plastic for use in bathyscapes instead of the steel capsules with viewports. In Professor Piccard's opinion, the wall of the capsule could be made thick enough to withstand safely a depth of 6,500 feet. Since he did not support the proposed concept of such a capsule with any experimental or analytical data, and very little was known about the behavior of acrylic plastic in complex biaxial and triaxial stress fields, the concept was considered on a par with science fiction. Because of the prevailing lack of interest in exploration of the oceans and the visionary nature of his proposal, the concept was not acted upon.

The next proposal for a spherical, transparent capsule was made by Perry ${ }^{2}$ in 1961 . He envisioned a transparent sphere assembled from two cast glass hemispheres joined by a mechanical lock located on the sphere's equator. According to him, such a capsule, when incorporated into a suitable submersible system, would permit the scientists to explore ocean depths in excess of 20,000 feet. The capsule would, according to him, withstand such depths

\footnotetext{
* For brevity, this and all further references to size will be understood to describe the outside diameter only.
} 
because of the very high compressive strength of glass, which also becomes more impact resistant as it is stressed during submergence in the ocean. This concept was supported by analytical calculations and exploratory experiments with small-scale glass spheres. Although this concept extends the depth range considerably beyond Piccard's and imposes extremely high-stress loading on the capsule material (glass), it has been favorably received. Since 1963 exploratory research has been conducted by the Navy to develop Perry's concept into operational hardware.

Piccard's and Perry's concepts were specifically oriented to depths beyond the continental shelf, and only to submarines or bathyscaphs. Neither of these two concepts answered the growing need for the exploration and utilization of the continental shelf by means of divers supported by specialized mechanical systems operated by nondivers enclosed in 1-atmosphere, pressureresistant capsules (Figure 1). To fill this gap, Navy scientists and engineers of the Naval Missile Center (NMC), Point Mugu, and the Naval Civil Engineering Laboratory (NCEL) ${ }^{3}$ proposed research that would result in a transparent capsule for manned operations at continental shelf depth within the shortest possible time. Immediate practical application of the transparent capsule concept would consist of developing a 66-inch man-size capsule and mating it with life-support and winch-down subsystems resulting in a bottom tethered manned observatory with vertical water column mobility (Figure 2). Besides serving as the focal point for the acrylic plastic capsule project, NEMO (Naval Experimental Manned Observatory)* was to be utilized by NCEL engineers as an in-situ underwater control center to supervise and counsel Seabee diver teams performing ocean engineering construction experiments on the continental shelf floor, while NMC scientists were to use NEMO as an in-situ observatory for the scuba diver and porpoise performance studies.

Since the overriding requirement for NEMO was that it become operational within the shortest span of time, past concepts for transparent capsules were reviewed with this requirement in mind. Perry's concept for a glass capsule was rejected because at that time there were no molds in this country capable of reliably producing large glass hemispheres, and it appeared that funding in excess of available exploratory funds would be required to design and purchase such molds, develop the casting process, and test a sufficient number of such capsules to establish confidence limits for their performance. Instead, Piccard's concept employing spherical acrylic plastic pentagons was chosen.

\footnotetext{
* Earlier designated Naval Edreobenthic Manned Observatory.
} 
The decision to select Piccard's concept had a sound engineering basis. First, considerably more is known about the engineering properties of acrylic plastic than those of massive glass. Much of this is data generated during the design of aircraft windows and canopies. 4,5 Second, acrylic plastic has already been successfully used in the construction of model-scale and full-scale pressureresistant capsules with predictable collapse pressures. ${ }^{6-10}$ Third, Commercial catalogs list acrylic plastic plate in sizes up to $4 \times 6$ feet and 4 inches thick which could be used for fabricating the spherical pentagon modules. F,ourth, a wealth of experience and equipment exists for machining, thermoforming, and bonding acrylic plastic.

However, even with all this information, there was not sufficient hardware-oriented data to design with confidence a man-rated acrylic plastic capsule. There was, for example, insufficient information on the compressive strength of acrylic plastic in biaxial and triaxial stress fields generated by longterm or cyclic load application. The data from the testing of acrylic plastic windows for aircraft could serve here only as a general guide since the data applied primarily to tensile stresses, rather than compressive stresses. However, because the strength of acrylic plastic is somewhat less in tension than in compression, much of the tensile stress data could serve as the first-order conservative approximation for prediction of magnitudes in compressive stresses and strains.

The available research findings from testing acrylic plastic capsules under external hydrostatic pressure described in detail the response of different acrylic plastic capsule designs to short-term loading. ${ }^{6-9}$ These findings do not cover long-term or cyclic loadings, which may cause the capsule to buckle through creep deformation or to fail by material fatigue, but they do indicate not only that Piccard's spherical pentagon concept is feasible, ${ }^{10}$ but also that pressure capsules based on any sound engineering design can be built successfully from acrylic plastic.

The experience in fabrication of acrylic plastic components accumulated by the industry pertained primarily to acrylic plastic stock less than 1 inch in thickness. Most of the techniques developed for thin acrylic plastic stock, except possibly for thermoforming and bonding, were applicable also to the thick stock that would be utilized in the full-scale capsule. Thermoforming posed special problems because the transfer of heat through thick acrylic plastic sections is very slow, and thus special procedures are required for heating and cooling. Bonding of thick curved sections also posed special problems because contactcementing large pressure hulls is not feasible and bonding of pressure hulls with a self-polymerizing cement had not been tried.

Thus, although some research data and experience in the application of acrylic plastic to the construction of pressure resistant structural components or whole structures existed, additional data and fabrication experience had to be generated before a man-size, acrylic plastic capsule could be designed and built for use at continental shelf depths. 


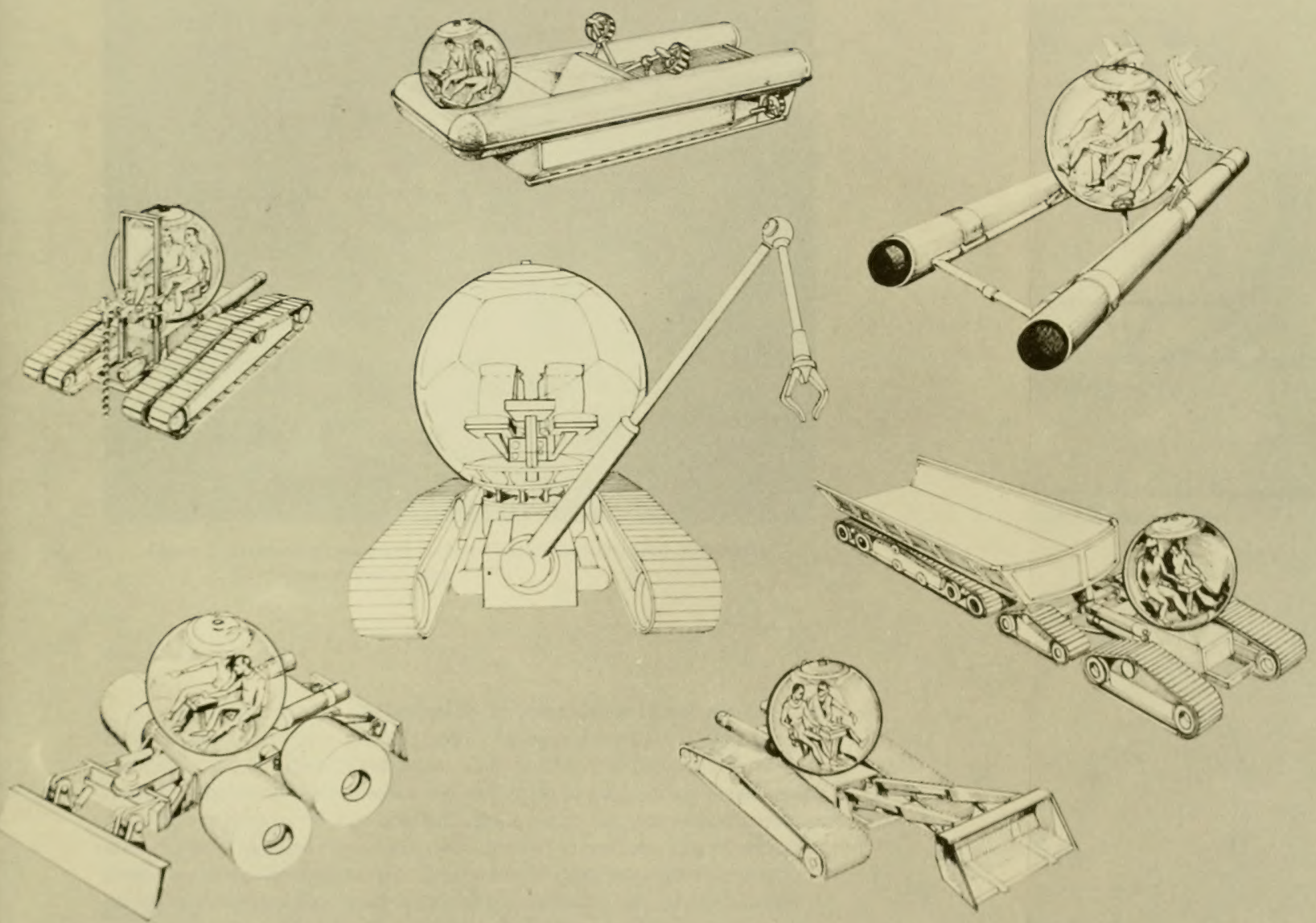

Figure 1. Typical applications for pressure-resistant acrylic plastic capsules in ocean engineering on the continental shelf. 



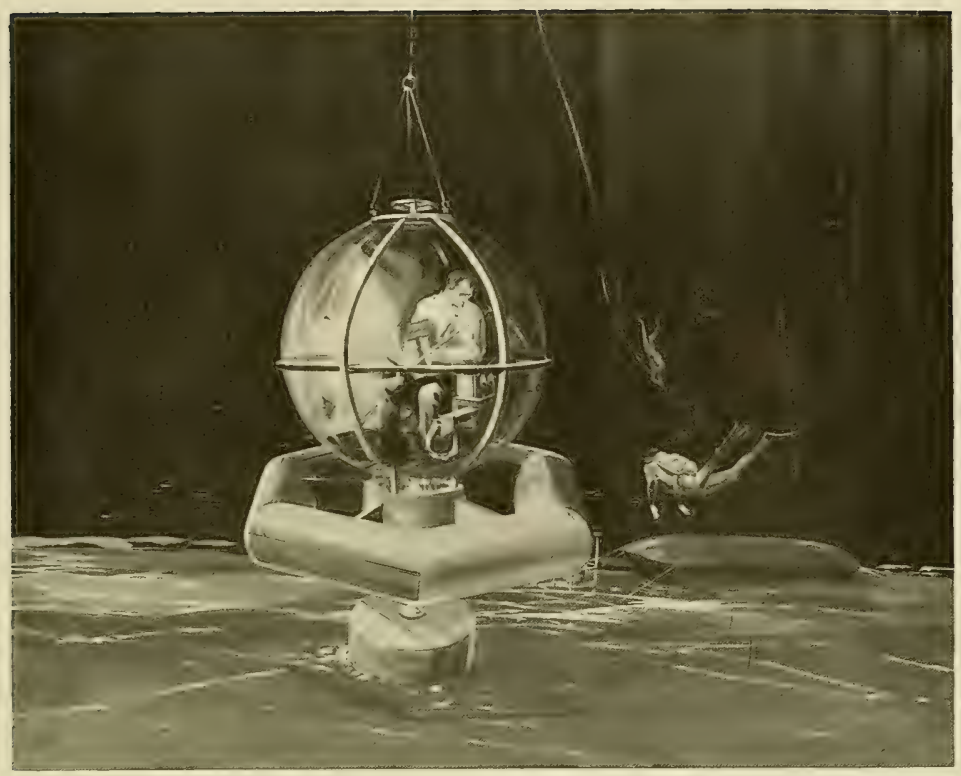

Figure 2. Engineering concept of the Naval Experimental Manned Observatory for continental shelf depths.

\section{Scope of Investigation}

The present study was limited to the investigation of a single 120-inch spherical capsule concept, grade $\mathrm{G}$ acrylic Plexiglas material, a single fabrication technique, and one operational depth. Only sufficient experimental investigations and analytical studies were to be performed to substantiate the adequacy of a single prototype 66-inch detailed capsule design for operation at continental shelf depth. The experimental data were limited to the recording of critical pressures, change of displacement, and strains of capsules under hydrostatic pressure. Analytical studies were limited to calculating critical pressure and strains under short-term loading, the only experimental condition for which analytical tools were in existence. 


\section{PROGRAM PLAN}

The research and development program for the acrylic plastic capsule was conceived to progress through five consecutive phases that upon their successful completion would result in a proven 66-inch-prototype capsule for incorporation into the operational winch-down NEMO system.

Phase 1 - Develop an engineering design that will permit using a spherical acrylic plastic capsule as a safe, man-rated, pressure-resistant hull.

Phase 2 - Develop a reliable fabrication process for the construction of small-scale as well as large-scale, acrylic plastic capsules according to the design of Phase 1.

Phase 3-Using scale models, evaluate experimentally (a) the design and fabrication process of the acrylic plastic capsule and (b) the performance of acrylic plastic as the main load carrying structural material.

Phase 4 - Validate the experimental data generated with small-scale models by fabricating and testing a large-scale prototype NEMO capsule.

Phase 5 - Develop a concept for the operational NEMO system that, besides the capsule subsystem, incorporates the life-support, winch, power supply, and control subsystems.

Since the successful completion of the study required facilities and personnel at NMC, Pacific Missile Range (PMR), and NCEL, a joint project was formulated between the three activities. The division of responsibility was according to area of specialization as follows:

$\begin{array}{lll}\text { Phase } 1 & \text { Design } & \text { NMC and NCEL } \\ \text { Phase } 2 & \text { Fabrication } & \text { PMR } \\ \text { Phase } 3 & \text { Model Tests } & \text { NCEL } \\ \text { Phase } 4 & \text { Prototype Tests } & \text { NCEL } \\ \text { Phase 5 } & \text { System Concept } & \text { NMC }\end{array}$

Since the study was exploratory with very modest funding, the coordination of the effort between the three activities was to be performed at the working level. All technical decisions were to be made on the basis of a consensus of engineering judgment expressed by the project personnel. 


\section{PHASE 1-DESIGN OF NEMO CAPSULE}

As mentioned previously, Piccard's concept ${ }^{1}$ was selected for the design of the capsule. However, since he described his concept only in very general terms, considerable detailing of his concept was necessary during the engineering design phase to insure the end product would be an operationally satisfactory capsule. Major omissions in the concept as stated by Piccard were: (1) the method for attaching the capsule to the submersible, (2) the method for incorporating electric and hydraulic feedthroughs in the capsule, (3) the method for attaching equipment to the capsule's interior, and (4) the method for fabricating the hull. In addition, some modifications to his concept had to be undertaken to reflect current operational requirements for pressure-resistant capsules.

\section{General Design}

Four major specifications of Piccard's concept were not modified and subsequently became the major constraints of the transparent capsule design for NEMO. They called for use of (1) acrylic plastic, (2) a spherical shape, (3) a monocoque stiffness system, and (4) structural modules in the form of spherical pentagons which, when assembled, form a sphere. The acceptance of these four general characteristics of the Piccard concept was not due to a desire to adhere to the original concept, but because these four characteristics reflect the results of an engineering optimization process in the selection of material, shape, stiffeners, and fabrication process.

For the continental shelf depth, acrylic plastic provided the most economical transparent material for the development of a man-rated capsule with satisfactory optical properties. Glass, although stronger in compression than acrylic plastic, appeared to require at least five times as much funding in order to obtain an operational man-rated capsule for continental shelf as was required for acrylic plastic.

Although there are many shapes for pressure-resistant hulls to choose from and several methods of stiffening them against implosion, the sphere remains the optimum shape. The monocoque hull design for stiffness is the most economical one for construction of spheres. ${ }^{11}$

Many fabrication processes could be employed to fabricate a monocoque acrylic plastic sphere, such as casting, drape forming, free forming, vacuum forming, male and female die pressing, vacuum-assist female mold forming, and others. The casting process could produce a hollow man-size , monolithic, monocoque sphere if the required substantial investment in molds, casting equipment, 
and casting process development was made. Other fabrication processes besides casting could be used to fabricate hemispheres which subsequently could be joined by bonding or by a mechanical lock. Casting is here again the only process available for producing hemispheres with the required thickness and out-of-roundness tolerances. Although the investment in hemispherical molds, casting equipment, and casting process development is less than for casting of monolithic spheres, it is still quite substantial.

It is only when the capsule can be assembled from many small structural modules that other fabrication processes can be effectively utilized to fabricate economically a monocoque sphere. Thus, instead of an expensive casting process, the much cheaper vacuum-assist mold forming can be applied to produce spherical sectors with uniform thickness and sphericity. The only limitation on this forming method is that the thickness to outside radius ratio $\left(t / R_{0}\right)$ be less than 0.125 for spherical sectors up to a 75 -degree spherical angle (as otherwise the temperatures required in forming become excessively high). (For larger sectors excessive variation in thickness also occurs due to unequal stretching of the acrylic plastic.) If the acrylic plastic sphere is broken down into 12 structural modules of pentagonal shape, the vacuum-assist, female mold forming technique becomes about the most economical fabrication process for spheres up to 6 feet in diameter and 4 inches in wall thickness (based on the $48 \times 60$-inch standard maximum size of acrylic plastic plates). Once the spherical sectors have been thermoformed, very little additional cost is required to machine them into pentagonal form. Spheres larger than 6 feet in diameter can be assembled from spherical pentagonal structural modules prepared by the vacuum-assist female mold form technique, but larger spheres would require use of premium-priced, oversize acrylic plastic plates. If procurement of oversize acrylic plastic plates is not feasible and $48 \times 60$-inch standard sheets are used, the structural modules would not be spherical pentagons of uniform size. The structural modules would either be spherical triangles of uniform size or they would vary both in configuration and in size. Thus, it can be seen that the spherical, pentagonal structural modules proposed by Piccard are, from the viewpoint of initial cost for fabrication equipment, fabrication process development, and fabrication process itself, the most economical approach to assembling a 66-inch monocoque sphere if thermoforming of flat acrylic plastic plates is substituted for casting of pentagons. Since the objective of the NEMO concept was to provide a reliable operational capsule for continental shelf depth, and not to set depth records, no effort was made to design the capsule for the 6,000-foot depth originally postulated by Piccard for his acrylic plastic capsule concept. Aside from the four major design constraints, all design parameters were to be determined during the course of the study. Thus, the resulting design would closely match the operational requirements of the NEMO system. 


\section{Detail Design}

Once the design parameters pertaining to material selection, shape configuration, operational depth, and assembly technique were selected, the detail design of the capsule could begin. The detail design was concerned with eight focal points:

(1) Means of ingress and egress for the capsule occupants

(2) Penetrations for electric and hydraulic conduits

(3) Attachment of the capsule to other structural systems

(4) Attachment of life-support equipment, chairs, and control console to the capsule interior

(5) Magnitude of permissible working stresses in the acrylic plastic and steel structural components

(6) Specification of construction materials

(7) Selection of hull thickness

(8) Structure and component dimensioning

Ingress and Egress. The access to the interior of the capsule could be achieved in several ways. The capsule could be separated into two hemispheres joined together by a mechanical lock that would permit opening of the top hemisphere for personnel access. Another approach to the same problem would consist of placing a hatch in the polar region of the sphere (Figure 3). The latter approach was chosen as it permitted egress from the capsule even when it was floating free on the ocean surface after emergency ascent without additional buoyancy systems. Although from a structural viewpoint an acrylic plastic hatch with a spherical bevel angle proposed by Piccard was the most desirable one (when closed it did not introduce any appreciable stress concentrations into the acrylic plastic hull), a metal hatch system with a spherical bevel angle was chosen for operational reasons (Figure 4).

The rationale behind this choice was that (1) an acrylic plastic hatch plug would soon lose its sealing ability because of surface scratches on the seal surface generated by equipment and personnel passing through the hatch opening, and (2) the metal would also act as efficient heat transfer surface to depress the temperature in the capsule's interior. Without such a cooling mechanism, pilots operating the capsule would soon become incapacitated by the heat generated in the capsule's interior. A metal hatch plug and matching ring insert would resist scratching more effectively and thus would prolong the operational life of the ingress-egress mechanism. 


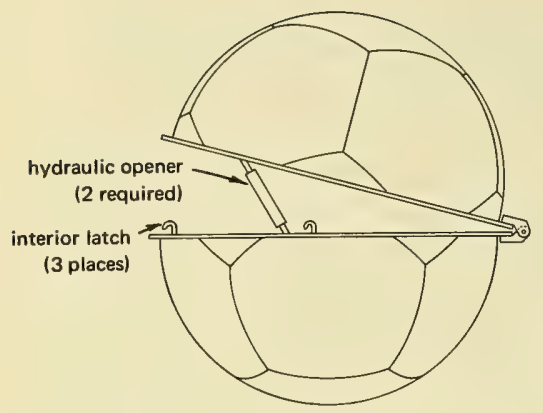

(a) Equatorial hatch.

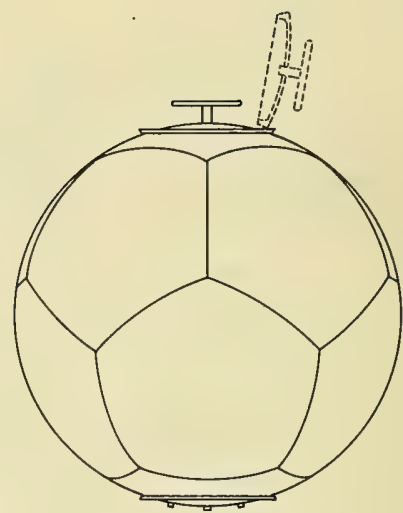

(b) Polar hatch.

Figure 3. Basic approaches for providing access to the interior of a spherical acrylic plastic capsule.

Since a metal hatch system constitutes a serious mismatch of rigidity with the acrylic plastic hull, a concentrated effort was made to reduce the thickness of the metal hatch to a minimum, so that the mismatch and resulting stress raiser effect could be minimized. The engineering decision was to reduce the thickness of the metal hatch to the minimum value corresponding in strength to implosion pressure of the acrylic plastic hull.

One of the problems facing the designer in providing a metal hatch for the capsule was to find a means for firmly attaching the beveled metal hatch insert ring to the acrylic plastic hull so that the whole hatch assembly would not fall out when the capsule was tossed around on the ocean surface. An ingenious solution was found: secure the hatch insert ring to the acrylic plastic hull by means of a metal retainer flange located on the interior of the hull to which the hatch insert ring would be bolted. Direct contact between the metal retainer flange and the acrylic plastic hull was considered undesirable as it would lead to scoring of the acrylic plastic, and local stress concentrations when the curvature of the capsule decreased during hydrostatic loading. To forestall these conditions, a compliant rubber gasket would be interposed between the metal retaining flange and the acrylic plastic hull. In addition, the retaining flange would be provided with a convex spherical surface matching the concave interior curvature of the capsule. The flange crosssection was to decrease towards its outer edge to make the flange more compliant at the outer edge than at the inner edge at the bolt circle. 


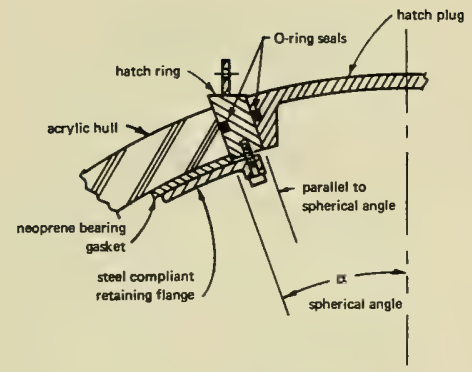

Hatch Closure

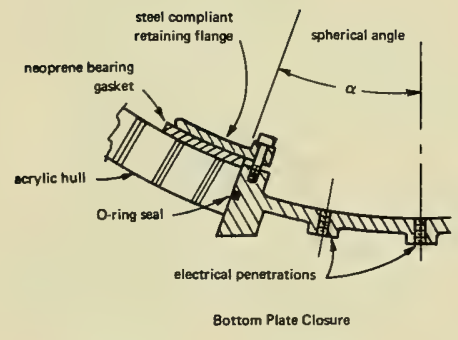

Figure 4. Hatch concept chosen for incorporation in the acrylic plastic capsule for the NEMO system.
Several sealing systems were considered for sealing the beveled hatch ring to the beveled bearing surfaces of the acrylic plastic capsule. Besides standard O-ring seals, rubber gaskets and epoxy adhesive were also considered. The O-ring seal was known to be reliable, but the groove in the metal would cause a contact stress raiser on the acrylic plastic bearing surface. A rubber gasket interposed between the metal and the acrylic plastic bearing surfaces would eliminate any stress concentrations, but would permit larger relative motion between the insert ring and the hull. Unless considerable force was constantly pressing the insert ring against the acrylic plastic, leakage would take place at the beginning of the dive until the capsule reached a substantial depth where hydrostatic pressure force would seal the hatch assembly against the acrylic plastic bearing surface.

Some thought was also given to using epoxy adhesive to bond the hatch insert ring in place permanently, thus eliminating both relative movement between

the parts and the leakage. This approach, however, was discarded as it was known that the magnitudes of forces acting across the metal-acrylic plastic interface would be so large that the adhesive would shear, permitting the water to leak in. Although the advantages and disadvantages of the O-ring and the gasket sealing systems are about equal, the O-ring system was chosen for the acrylic plastic capsule because it utilized primarily off-the-shelf seals. If the stress raiser problem proved troublesome later, the iubber gasket system could be substituted. 
Although the hatch system requires some arrangement for opening and locking the hatch by the occupants of the capsule, no thought was given to this problem during initial planning, because it was thought that any locking arrangement used in submersibles or by the chemical industry in autoclaves would be satisfactory for the NEMO capsule and would have very little if any bearing on the stress distribution in the hatch assembly or in the acrylic plastic hull.

Penetrations. Penetrations for electrical and hydraulic conduits represented a serious problem as they are sources of serious stress concentrations. Rather than have them distributed throughout the pressure hull, as is customary in steel pressure-resistant capsules, they were all placed in a single metal plate insert (Figure 4). This eliminated several design problems. First, a great number of penetrations in an acrylic plastic hull with the accompanying stress concentrations were replaced by a single penetration with a stress concentration approximately equal to that of the hatch. Second, placing all the penetrations associated with electrical and hydraulic feedthroughs in a metal plate eliminated all problems associated with drilling and tapping acrylic plastic. And third, placement of the individual penetrations in the metal plate made the incorporation of reinforcements around the many penetrations a relatively easy problem to solve. This was accomplished by placing the penetrations in a thicker section of the plate, where the effect of stress concentrations would not necessarily initiate failure of the whole plate. The location of the plate itself was optional, depending on other factors like (1) method of attaching the capsule to the submersible's exostructure and (2) method of attaching the life support, seats, and control console to the capsule.

The same method of sealing and attaching the penetrator plate to the acrylic plastic hull was to be utilized as for the hatch insert ring. Furthermore, by choosing the same diameter for the penetrator plate as for the hatch insert ring, no additional detail design would be needed for the seal and retaining flange.

External Attachment of Capsule. The attachment of the capsule to other structural systems posed a difficult problem to which several solutions were considered. The difficulty of attaching the capsule to another structural system lay in several design and operational constraints that had to be satisfied by the proposed attachment method. The design constraints that had to be satisfied were (1) no direct attachment to the acrylic plastic hull by means of steel bolts or screws, (2) no restraint was to be imposed on the decrease of capsule diameter during pressure loading, (3) the attachment was to be stiff enough to prevent excessive movement of the whole capsule with respect to the framework during raising or lowering of the NEMO structure in the ocean, (4) retention of as much panoramic visibility as possible for the capsule even 


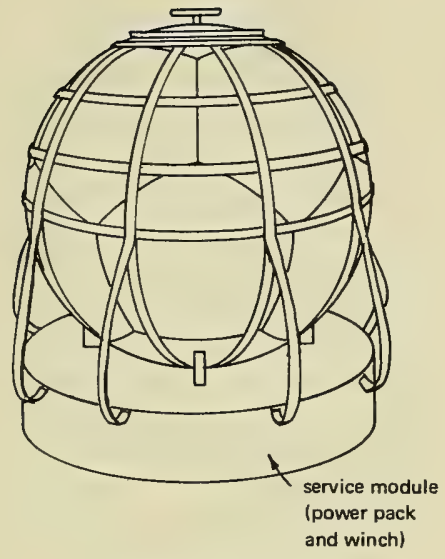

(a) Resting on deck.

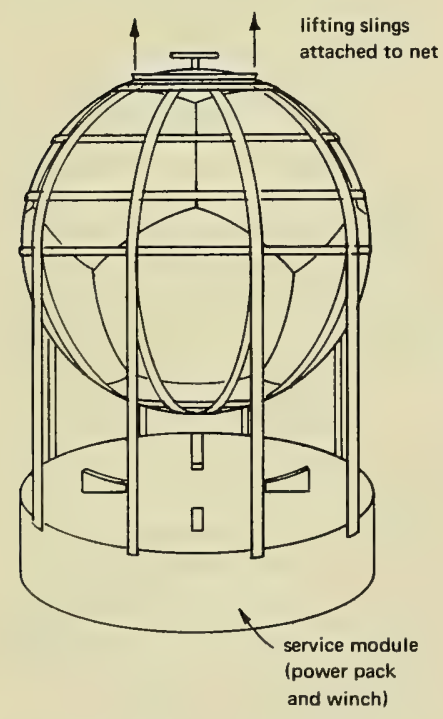

(b) Being lifted off the deck.

Figure 5. Net restraint concept for attaching the acrylic plastic capsule to the NEMO service module. in the presence of structural members serving as the attachment, (5) the attachment was to work equally well regardless of whether the capsule was on deck, being lifted, floating on the ocean surface, or being winched down to operational depth, and (6) the attachment subsystem was not to generate excessive tensile stresses in the acrylic plastic capsule that could lead to a failure of a bonded adhesive joint whose minimum strength is approximately $40 \%$ less than of the parent material in tension.

Several design approaches were tried to arrive at a satisfactory attachment between the acrylic plastic sphere and the remainder of the NEMO structure. The most obvious solution to the problem, the placement of the capsule in a net (Figure 5), similar to the nets used for hanging gondolas under spherical balloons, had been immediately discarded as it did not satisfy three of the design constraints. Such a net would considerably restrict the panoramic visibility, it would permit excessive relative motion between the sphere and the remainder of the NEMO structure thus imposing severe stresses on hydraulic and mechanical control linkages between the control console inside the capsule and the operational components, and it would not provide any support for the capsule when it was being transported on land, or resting on deck of the ship. 


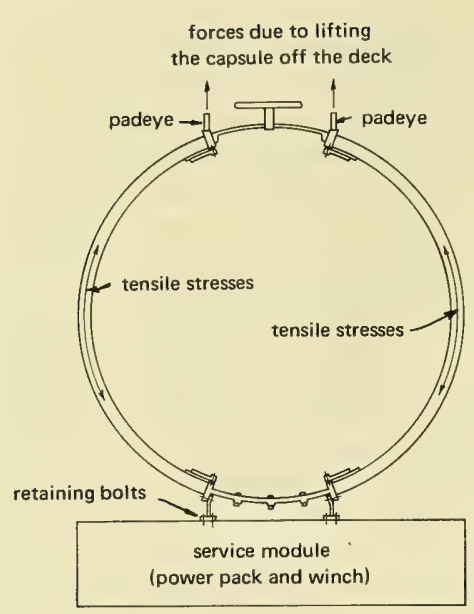

Figure 6. Bolt-on restraint concept for attaching the acrylic plastic capsule to the NEMO service module.
Another approach that was investigated and subsequently discarded was to attach the capsule to the service module by bolting on the metal penetrator plate where all of the feedthroughs were located (Figure 6). For this purpose, the penetrator plate would have to be located at the bottom of the capsule so that it could resist best the upward force generated by the buoyancy of the capsule when attached to the negatively buoyant service module. Such an attachment would possess some good points: (1) rigidity, (2) equal resistance to upward and downward forces, (3) ease of design and fabrication, and (4) unobstructed visibility. It has, however, two serious disadvantages: (1) it would generate considerable tensile stresses in the acrylic plastic joints when the NEMO system is lifted

out of water by means of a line attached to the top hatch insert ring, and (2) it would generate flexure stresses in the acrylic plastic capsule when underwater currents impinge upon the capsule rigidly attached to the service module, thus creating a moment loading on the capsule. Of these two disadvantages, the first is the more serious.

Analysis of the shortcomings of the rigid attachment to the metal penetrator plate provided an approach to the problem that eliminated all the bad features of the previous design approach and retained all the good ones. In this design approach, the capsule was attached by bolting the metal penetrator plate to the service module, but the tensile stresses across the bonded acrylic plastic joints generated by hoisting operations were eliminated by the incorporation of pretensioned tie rods connecting the hatch insert ring to the penetrator plate located at the opposite pole of the capsule (Figure 7). With such an arrangement, the upward force applied to the hatch insert ring is transmitted to the service module through the tie rods rather than through the acrylic plastic hull. The incorporation of pretensioned tie rods introduces an additional compressive loading on the acrylic plastic hult when it is on deck, floating on the surface, or shallowly submerged. Because the tie rods are under tension, the acrylic plastic hull is placed under diametrical compression. If the 


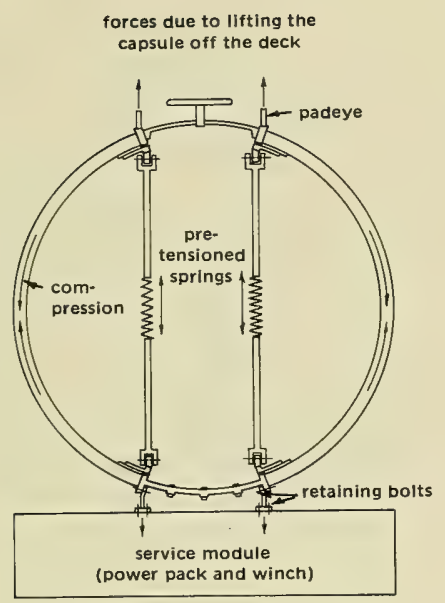

Figure 7. Tie-rod restraint concept for attaching the acrylic plastic capsule to the NEMO service module. magnitude of pretension selected for the tie rods is larger than the forces applied to the hatch system during lifting, the joints in the acrylic plastic hull will never be subjected to tensile stresses due to lifting and will generally be under compression. In this manner, the danger of joint failure will be completely eliminated, as under compression the joints are self-wedging without any shear stresses at the joint surfaces. In the design of the pretensioned tie rods, the shrinkage of the capsule under hydrostatic loading at design depth had to be taken into account. If designed properly, the tie rods would be under the needed tension while the NEMO system is out of the water, while at design depth the tension would approach zero. In this manner the diametral compression of the

capsule by tie rods will not be superimposed on the hydrostatic compression at design depth thus eliminating the danger of triggering the elastic collapse of the capsule by the localized tie-rod pretensioning forces. To achieve such a self-adjusting pretensioning system, a spiral spring with extension equal to diametral shrinkage of the capsule would have to be incorporated into each tie rod. The total force exerted by the springs in the tie rods while on deck would be equal to the buoyancy of the NEMO capsule while at the design depth it would decrease to almost zero.

The tie-rod system appeared to be an adequate solution to the attachment problem so long as the presence of several tie rods in the interior of the capsule was not considered objectionable. Since for some capsule sizes or possible non-NEMO system applications the presence of tie rods may be objectionable, additional approaches were considered that did not require the presence of tie rods. One of such proposed approaches utilized an external cage. To eliminate the tensile stresses associated with lifting of the NEMO system out of water or when it just floats on the ocean surface with the negatively buoyant power module pulling downward, an external rigid cage was added to the NEMO system (Figure 8). The point of contact between the NEMO capsule and the rigid cage was to be only at the polar metal plates 


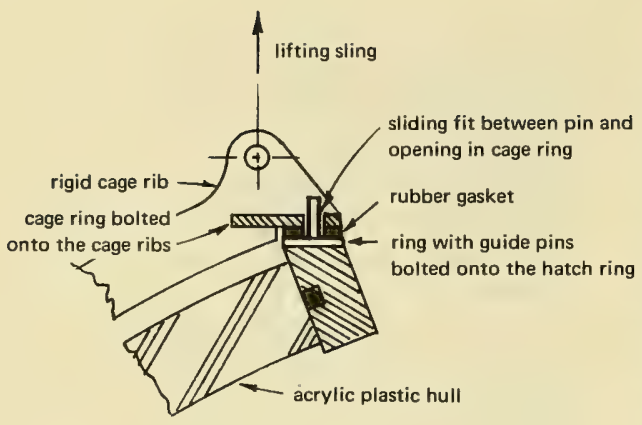

Detail

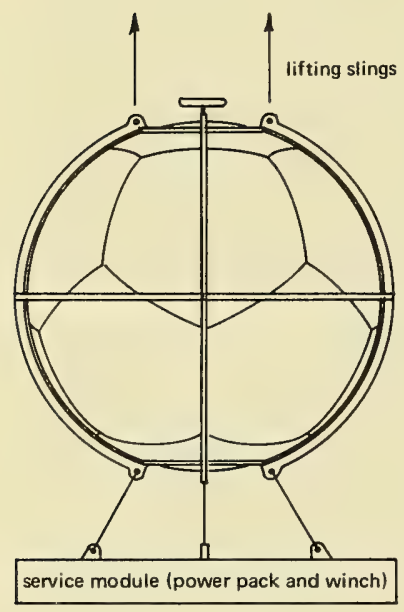

Figure 8. Rigid cage restraint concept for attaching the acrylic plastic capsule to the NEMO service module. in the capsule. Since a rigid attachment to the cage at both top and bottom steel inserts was undesirable, as it would restrain the sphere radially during pressure application, a special joint was devised that permitted the sphere to change its radial dimension but at the same time resisted lateral forces applied to the sphere by waves on the ocean surface, or currents when submerged. The joint devised for this application consisted basically of pins attached to the polar metal plates that fitted into oversize holes in the cage framework. Thus, the sphere was permitted to change its diametrical dimension while at the same time the pin and hole joint restrained the whole capsule from lateral or vertical displacement that would cause it to strike the ribs of the cage. The sliding pin joint imposes only lateral and ver. tical but not diametral restraint. Because of this, no external tensile loading can act on the capsule, regardless whether the NEMO system is being lifted out of water or just floating on the ocean surface. The drawback of this capsule attachment to the NEMO service module was that the cage did reduce to some extent the panoramic visibility of which an acrylic plastic capsule is capable.

Because of it, this attachment arrangement would not be as satisfactory from the optical viewpoint as the tie-rod attachment. There was, however, a compensating feature to the cage. If the cage members were spaced appropriately the cage could act as a protector against impacting the ship during launching operations. This protective feature of the cage was felt to compensate adequately for the loss of visibility caused by the cage. 


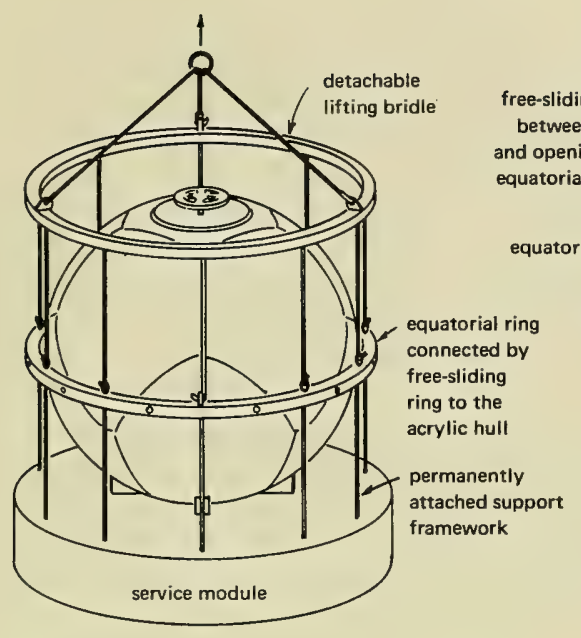

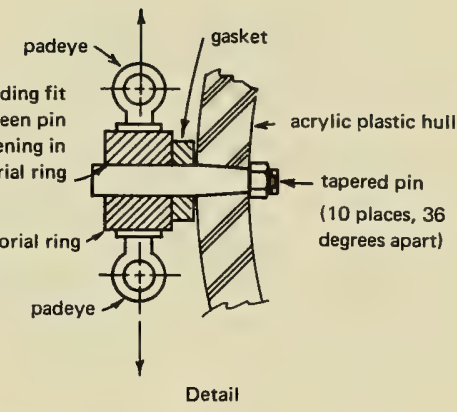

Figure 9. Equatorial ring and pin restraint for attaching the acrylic plastic capsule to the NEMO service module.

Another approach that was considered was an equatorial ring held in place by radial sphere penetrators (Figure 9). The sphere penetrators were to be tapered and made from plastic with elastic properties matching those of the acrylic plastic hull so that no stress raiser effect would be introduced by them. The fit between the pins and the matching holes in the equatorial ring was to permit sliding of the pins when they move radially inward with the shrinking capsule under hydrostatic loading. The capsule held by the equatorial ring basically would not be subjected to tensile loading as the major forces generated by lifting of the NEMO system out of water would be shunted around the acrylic plastic capsule via the lifting attachments to the equatorial ring. The only tensile loading acting on the acrylic plastic capsule would be caused here by the dead weight of the capsule suspended from the plastic penetrator pins when being lifted out of water. When floating on the ocean surface, there would be some tensile stresses in the hull, but they would be well distributed around the hull circumference by the radial sphere penetrators in the equatorial ring. In view of the rather low weight of the capsule and the large wall cross section, the tensile stresses generated by it would be low. Although the equatorial ring method of attaching the capsule to the NEMO service module is not particularly advantageous, it may become so if the NEMO capsules become part of some other system in which it is important to have the capsule attached to the submersible at its equator rather than at the poles as it is in the NEMO system. 
Attachment of Equipment to the Interior of the Capsule. The interior of the capsule must contain certain subsystems that are needed to support the life of the crew and to allow them to control effectively the operation of externally located subsystems. These subsystems give the capsule vertical or horizontal mobility, and possibly the ability to perform work by the use of external manipulators. As a minimum, such subsystems located in the interior of the capsule would consist of life-support equipment, seats, control console, emergency power supply, communication gear, and cameras. All of this equipment must be securely attached to some rigid support that would prevent it from being tossed around during launch and retrieval operations on the ocean surface.

When pressure-resistant capsules are of steel, securing such equipment poses no design problem as the necessary brackets or framework can be directly attached to the steel capsule by means of bolts or welding. A different case presents itself for the acrylic plastic capsule. Since welding or bolting to the interior capsule wall is not permissible, other ways had to be considered for the securing of equipment. After considerable thought was given to this problem, four different approaches were arrived at for securing of equipment on the interior of the capsule:

1. The equipment was to be bolted to a floor that in turn was bonded to the wall of the capsule by means of a foamed-in-place plastic that completely filled the spherical sector space enclosed between the flat circular floor and the wall of the capsule below (Figure 10). The foamed-in-place plastic, for example styrofoam, would distribute evenly in the capsule the weight of the equipment and crew resting upon it. Because of the low modulus of elasticity of such material, it would permit the diameter of the acrylic plastic capsule to shrink considerably under hydrostatic loading without imposing a noticeable restraint. The disadvantage of this arrangement lay in the permanency of the foamed-inplace filler. Once the cavity below the floor was filled with foamed plastic, it would become very difficult to perform any maintenance or modification to the wiring and plumbing located below the floor.

2. The equipment and the floor were to be bolted to an internal cage that snugly fitted the interior dimensions of the acrylic plastic capsule (Figure 11). Elastomeric spacers were to be inserted between the ribs of the cage and the inner surface of the acrylic plastic capsule to permit the shrinkage of the capsule's internal diameter without transfer of load to the cage. The major shortcomings of this attachment method were the restriction of panoramic vision, difficulty of assembling such a cage in the cramped interior of the capsule from structural members small enough to pass through the hatch, and reduction of usable interior space. 
3. The equipment and the floor were to be attached to the pretensioned tie rods (Figure 12). For such an arrangement, the tie-rod springs would be located near the top hatch so that the portion of the tie rods to which the floor and equipment were attached would not experience any displacement relative to the bottom metal plate to which hydraulic tubing and electrical leads were attached. The disadvantage of this arrangement lies primarily in the difficulty of packaging equipment with respect to the tie rods. Furthermore, problems would be encountered in the removal of equipment for maintenance and modification as in many cases the tie rods would have to pass through the equipment in order to exert proper vertical and lateral restraint.

4. The equipment was to be bolted to the floor, which in turn would be bolted to the bottom metal plate insert (Figure 13). Since the diameter of the floor is larger than that of the bottom metal plate, it cannot rest directly on the bottom plate but must be held some distance above the plate. To avoid having the floor rest on the compliant acrylic plastic hull while it is restrained by bolts to the rigid bottom metal plate, a spacer has been provided that keeps the floor a fixed distance above the bottom steel plates. A small clearance is provided between the edge of the floor and the inner wall of the capsule to allow for the shrinkage of the sphere without imposition of restraint on the sphere and compressive loading on the floor. Since all the equipment, occupants, and the floor rest on top of the bottom metal plate, approximately a 1,000-pound force acts upon it. Whether this downward-acting force generates tensile stresses across the bonded joints between individual pentagons depends on the method used for attaching the capsule to the NEMO exostructure and whether the capsule rests on deck, is being lifted, floats on ocean surface, or is submerged at its operational depth. When pretensioned tie rods are used on the interior of the capsule, and the bottom metal plate is rigidly attached to the ballast pod, no tensile stresses can be generated by the weight of the equipment in the interior of the capsule regardless of the stage of launch or retrieval operations. When the capsule is held in place by the external cage with sliding pin joints, tensile stresses are generated in the bonded joints when the capsule floats on the ocean surface. At that time, the bottom metal plate is not being supported by the external cage since the capsule has lifted off its bottom support in the cage and is pushing against its top seat in the cage. Furthermore, the hydrostatic pressure acting on the bottom pentagon from outside is not high enough to equal the downward force exerted from inside on the plate by the weight of equipment located in the capsule. Thus, the resultant force that is equal to the difference between (1) the sum of the weights of bottom metal plate, polar pentagon, and internal equipment and (2) the upwardacting hydrostatic pressure on the bottom pentagon generates tensile stresses 
in the joints between the bottom polar pentagon and the adjacent pentagons. When the NENO system is submerged more than 20 feet underwater, the upward acting hydrostatic pressure overcomes the downward acting weights of bottom plate and internal equipment relieving all the tensile stresses in the joints between individual spherical pentagons.

Working Stresses. The selection of a working stress level is always a very crucial engineering decision for internal or external pressure hulls whose catastrophic failure would kill the operating personnel. When the material selected has not been used previously for construction of pressure hulls, selection of a working stress level becomes highly subjective. The designer must draw heavily on experimental data peripheral to the problem at hand and on his engineering judgment.

Since acrylic plastic pressure hulls had not been designed or built previously for manned operation, no precedent existed on which to draw. There existed, however, two sources of experimental data that impinged on the design of acrylic plastic capsules. One was the body of experiments conducted over a period of years for determination of allowable stresses in acrylic plastic windows and canopies for aircraft. ${ }^{4,5}$ The other source of data was the evaluation of acrylic plastic windows for undersea applications performed by NCEL and other laboratories. ${ }^{12-15}$

Although a wealth of experimental data has been generated by engineers during design and evaluation of aircraft windows and canopies, little of it is directly applicable to the design of an acrylic plastic pressure capsule. The windows and canopies in aircraft are (1) in tension and (2) cannot fail by elastic or plastic instability, while the acrylic plastic capsule is (1) in compression and (2) it generally fails by elastic or plastic instability. However, the aerospace research program provided the following information* relevant to the selection of working stress levels for the acrylic plastic capsule:

1. Under short-term tensile cyclic loading at a 6,000-psi stress and a $77^{\circ}$ temperature level, the life of acrylic plastic is about $10^{4}$ cycles.

2. Under long-term tension at a 6,000-psi stress and a $77^{\circ}$ temperature level, the acrylic plastic will rupture only after about 48 hours of sustained loading. At a 4,000-psi stress level, the material will not fail in less than 1,000 hours.

\footnotetext{
- Based on MIL-P-5425A and MIL.P-21105C materials.
} 


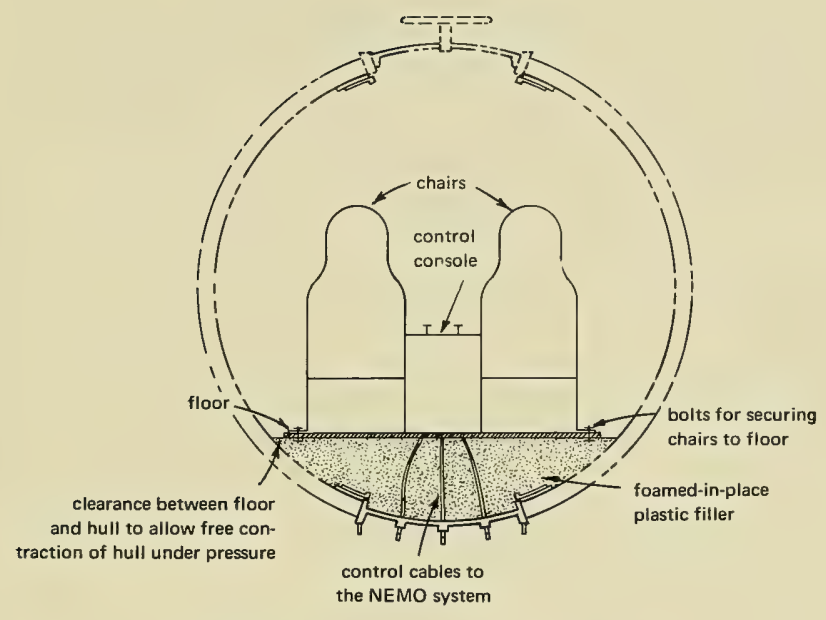

Figure 10. Foamed in-place restraint for securing of equipment inside the acrylic plastic capsule.

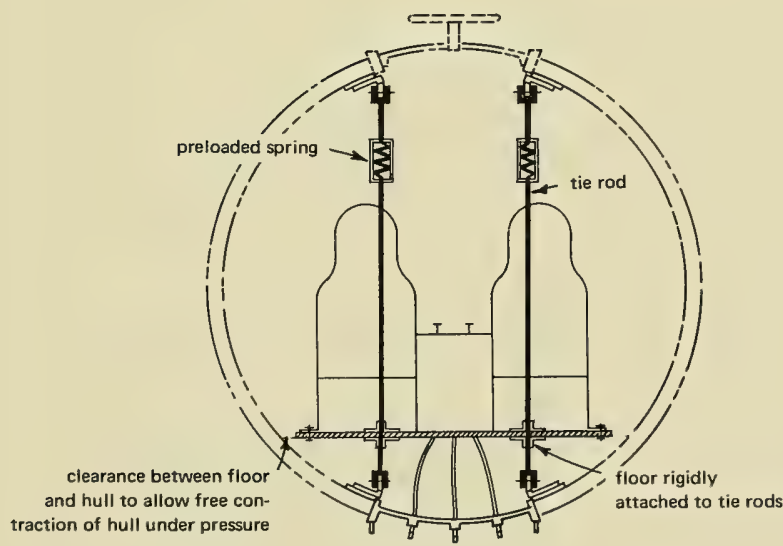

Figure 12. Tie-rod restraint for securing equipment inside the acrylic plastic capsule.

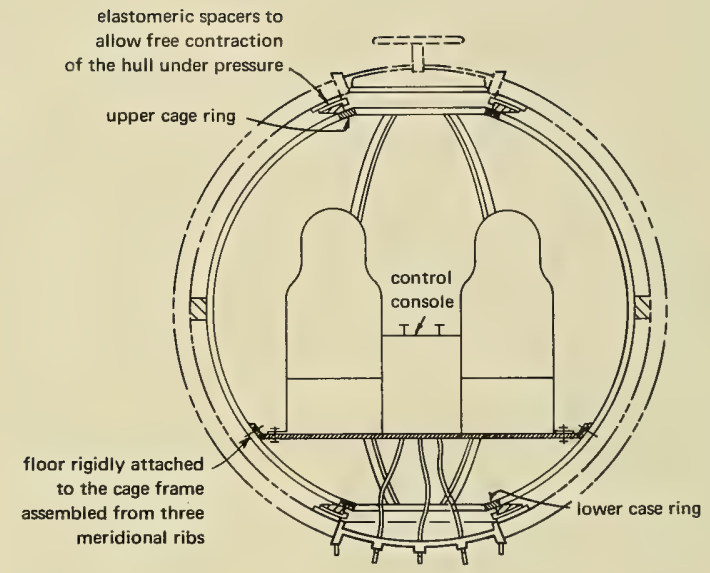

Figure 11. Internal cage restraint for securing equipment inside the acrylic plastic capsule.

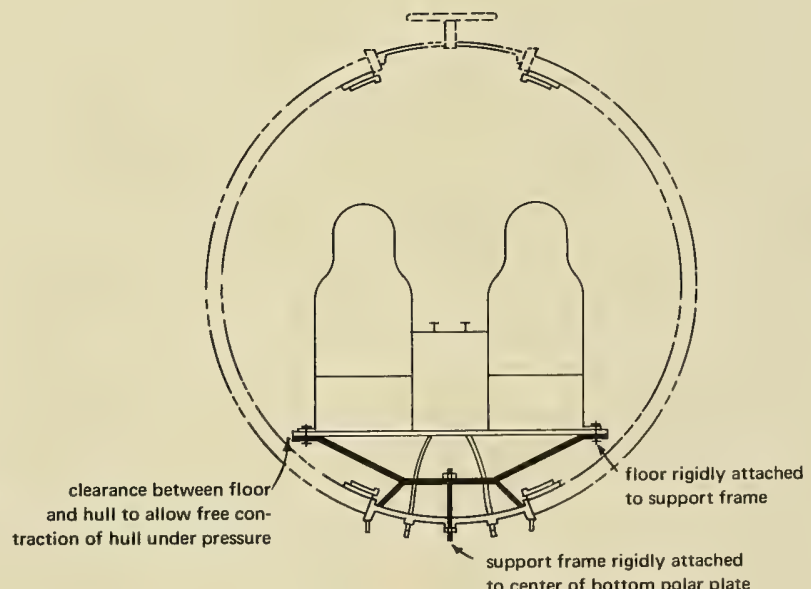

Figure 13. Polar plate restraint for securing equipment inside the acrylic plastic capsule. 

3. The secant modulus of elasticity of acrylic plastic after 48 hours of sustained tensile loading in the 75 to $77^{\circ} \mathrm{F}$ range and at a 6,000-psi stress level is 90,000 psi; at a 4,000-psi stress level it is 200,000 psi; at a 3,000-psi stress level it is 300,000 psi; and at a 2,000-psi level it is 400,000 psi.

4. The strength and modulus of elasticity increase as the temperature of acrylic plastic is decreased from room temperature ( 75 to $77^{\circ} \mathrm{F}$ range). The increase is about $10 \%$ for the 30 to $35^{\circ} \mathrm{F}$ range.

5. There is no change in the impact strength of unnotched acrylic plastic bars with the decrease in temperature from the 75 to $77^{\circ} \mathrm{F}$ range to 30 to $35^{\circ} \mathrm{F}$ range.

6. The secant modulus of elasticity of acrylic plastic under sustained compressive loading in the 75 to $77^{\circ} \mathrm{F}$ range is 10 to $20 \%$ higher than for sustained tensile loading.

7. The compressive short-term strength of acrylic plastic is approximately 15,000 psi, while the tensile strength is 9,000 psi.

The data generated in the NCEL hydrospace window research program was less voluminous than in the aerospace research program but it had the advantage of being more directly applicable. In this research conical, flat disc, and spherical acrylic plastic windows were subjected primarily to compressive stresses, which either caused the windows to fail because of excessive stress magnitude or plastic instability. The results from the hydrospace window research program applicable to the capsule design were:

1. Time-dependent ultimate failure of acrylic plastic structural components by viscoelastic or viscoplastic instability caused by sustained compressive loading occurs in less than 24 hours only when the loading level is generally $\geqslant 70 \%$ of the short-term compressive strength. When the loading is $\leqslant 25 \%$, the ultimate failure will definitely not occur in 10,000 hours.

2. Cyclic compressive loading may cause ultimate fatigue failure of acrylic plastic structural components in less than 100 cycles only when the loading is $>50 \%$ of short-term compressive strength. When the loading is $\leqslant 25 \%$, the ultimate failure will definitely not occur in 1,000 cycles.

From the rather general and only indirectly applicable information available at the time of design of the NEMO acrylic hull, two general conclusions could be made:

1. If the maximum operational depth of the hull was chosen to be at 20 to $35 \%$ of its implosion depth under short-term loading conditions the hull would probably be safe for long-term cyclic and sustained pressure service at the maximum operational depth so long as the fatigue life was not to exceed 1,000 dives of 8 -hour, or 100 dives of 100-hour duration. 
2. Since the low temperatures that commonly exist in the ocean at continental shelf depth make the test results obtained in the laboratory at room temperature $\left(75\right.$ to $77^{\circ} \mathrm{F}$ ) conservative for the design of hulls that will operate in the cooler ocean waters, there is no reason to conduct capsule evaluation tests under simulated ocean temperature, as lower temperatures are operationally much more difficult to maintain over long periods of time in pressure test facilities than room temperature.

Since the objective of the acrylic plastic pressure capsule program was to provide an operational system for continental shelf depth, the maximum operational depth had to be in the 600-to-1,000-foot range. The working stress in the hull had to be selected accordingly either for the 600 - or the 1,000-foot operational depth. Because the exact relationship between the working stress level and the cyclic life of the acrylic plastic hull was not known, it was decided to select the greater depth of 1,000 feet as the maximum operational depth. A 1:3 relationship between the maximum working depth and the implosion depth under short-term loading was selected; implosion depth could be approximated with existing equations. In this manner, if the working stress corresponding to a 1,000-foot depth did not cause premature fatigue failure, the hull would be rated for a 1,000-foot operational service. If, on the other hand, the working stresses at a 1,000-foot operational depth caused premature cyclic failure (that is, in fewer than 1,000 dives to 1,000-foot depth) of the hull, then the working stress could be lowered by changing the operational depth of the hull from 1,000 feet to 600 feet. Since the 600 -foot operational depth was also acceptable, such an arrangement was considered to be satisfactory and the working stress level was chosen to be $1 / 3$ of the stress level associated with the calculated implosion pressure of the capsule under short-term loading. The working stress level was chosen on the basis of the relationship with the short-term hydrostatic loading of the capsule to implosion rather than on the basis of relationship with ultimate failure of an acrylic plastic test specimen under uniaxial compression because the fact that plastic instability must be considered in the evaluation of capsule failure. The compressive test of an acrylic plastic specimen does not take this into account.

In addition to selecting the working stress for the acrylic plastic, the appropriate working stress had to be selected for the metal hatch and bottom insert plate. The problem here was considerably simpler. Metals such as steel, titanium, or aluminum do not exhibit viscoelastic or viscoplastic behavior in the 32 to $120^{\circ} \mathrm{F}$ temperature range, and the response of metals to different compressive or tensile stress levels is well understood. Thus the selection of working stress here was more of a design than research problem. The metal hatch and polar plate could be designed to fail at higher 
hydrostatic loading than the acrylic plastic hull, at equal loading, or at lesser loading. Designing it to fail at lower pressure than the hull would lower the overall safety of the capsule while designing it to fail at pressures above that of the acrylic plastic hull did not raise its overall safety, so a working stress level was chosen for metallic hull components that would cause them to fail at the same depth as the acrylic plastic capsule under short-term hydrostatic loading.

Specification of Materials. Although the decision had been made early in the research program to utilize acrylic plastic as the transparent hull material and metals for hatches and bottom penetration plate, a set of specifications had to be drawn up for their procurement.

Several grades of acrylic plastic are commercially available in the form of sheets and plates. The acrylic plastic is available in heat-resistant, unshrunk, utility grade (MIL-P-21105C); heat-resistant, preshrunk, optical grade (MIL-P-5425); modified cross-linked, preshrunk, optical grade (MIL-P-8184); and modified cross-linked, crack-propagation-resistant, prestretched optical grade (MIL-P-25690).

Since for the safe performance of the acrylic plastic capsule the most important material properties are compressive strength, shear strength, tensile strength, impact resistance and time-dependent viscoelastic or viscoplastic deformation, all of the existing acrylic plastic grades were carefully examined to determine which one of them best satisfied the material property requirements. A review of the material properties applicable to the NEMO capsule showed, however, that there was very little significant difference between the different grades of acrylic plastic materials with the possible exception of the stretched acrylic plastic that possesses significantly higher impact strength than all the other grades. A survey of acrylic plastic sheet producers revealed, however, that only MIL-P-21005C is available as a standard stock item in thicknesses to 4 inches; MIL-P-5425 could be obtained as a special order stock item at premium price from the suppliers, while the modified and prestretched acrylic plastic was simply not produced in the thicknesses above 1 inch. Further examination of standard commercial MIL-P-21105C plates of up to 4-inch thicknesses revealed that their optical properties are more than adequate for visual observation of hydrospace. Since MIL-P-21105C satisfied the operationally required optical criteria, was readily available in thicknesses to 4 inches, was the least expensive of all acrylic plastic material grades, and at the same time could satisfy the structural requirements of the capsule, it was chosen as the capsule material. The mechanical and physical properties of the heat-resistant, unshrunk, utility grade acrylic plastic (MIL-P-21105C) and the ASTM tests used in determining them are shown in Table 1. 
Table 1. Physical and Mechanical Properties of Grade G Plexiglas Acrylic Plastic Used for Fabrication of NEMO Hulls

\begin{tabular}{|c|c|c|}
\hline \multicolumn{3}{|c|}{ Physical Properties } \\
\hline Property & Typical & Test Method \\
\hline Hardness, Rockwell M & 90 & ASTM-D785-62 \\
\hline Hardness, Barcol & 90 & ASTM-D2583 \\
\hline Specific gravity & $\begin{array}{c}1.19 \pm 0.01 \\
(2 \text { tests within } 0.005)\end{array}$ & ASTM-D792-64T \\
\hline Refractive index; $1 / 8$ inch & $1.50 \pm 0.01$ & ASTM-D542-50 \\
\hline Luminous transmittance; $1 / 8$ inch & $91 \%$ & ASTM-D1003-61 \\
\hline Haze, $1 / 8$ inch & 2.3 & ASTM-D1003-61 \\
\hline $\begin{array}{l}\text { Heat distortion temperature } \\
+3.6^{\circ} \mathrm{F} / \text { minute at } 264 \mathrm{psi} \\
+3.6^{\circ} \mathrm{F} / \text { minute at } 66 \mathrm{psi}\end{array}$ & $\begin{array}{l}200^{\circ} \mathrm{F} \\
220^{\circ} \mathrm{F}\end{array}$ & $\begin{array}{l}\text { ASTM-D648-56 } \\
\text { ASTM-D648-56 }\end{array}$ \\
\hline Thermal expansion $/{ }^{\circ} \mathrm{F}$ at $20^{\circ} \mathrm{F}$ & $35 \times 10^{-6}$ & $\begin{array}{l}\text { Fed. Std } 406 \\
\text { Method } 2031\end{array}$ \\
\hline $\begin{array}{l}\text { Water absorption; } 1 / 8 \text { inch } \\
24 \mathrm{hr} \text { at } 73^{\circ} \mathrm{F} \\
\text { To saturation }\end{array}$ & $\begin{array}{l}0.3 \% \\
1.9 \%\end{array}$ & $\begin{array}{l}\text { ASTM-D570-63T } \\
\text { ASTM-D570-63T }\end{array}$ \\
\hline \multicolumn{3}{|c|}{ Mechanical Properties } \\
\hline Property & Minimum Requirement & Test Method \\
\hline $\begin{array}{l}\text { Tensile strength, rupture } \\
\text { (0.2 in./minute) }\end{array}$ & 9,000 psi & ASTM-D638-64T \\
\hline Tensile elongation, rupture & $2 \% \min$ to $7 \% \max$ & ASTM-D638-64T \\
\hline Modulus of elasticity, tension & $400,000 \mathrm{psi}$ & ASTM-D638-64T \\
\hline $\begin{array}{l}\text { Compressive strength, yield } \\
(0.2 \text { in./minute) }\end{array}$ & 15,000 psi & ASTM-D695-63T \\
\hline Modulus of elasticity, compression & 420,000 psi & ASTM-D695-63T \\
\hline Flexural strength, rupture & $14,000 \mathrm{psi}$ & ASTM-D790-63 \\
\hline Shear strength, rupture & $8,000 \mathrm{psi}$ & ASTM-D732-46 \\
\hline $\begin{array}{l}\text { Impact strength, } 1 \text { zod } \\
\text { (per inch of notch) }\end{array}$ & $0.4 \mathrm{ft}-\mathrm{lb}$ & ASTM-D256-56 \\
\hline $\begin{array}{l}\text { Compressive deformation under load } \\
\left(4,000 \text { psi at } 122^{\circ} \mathrm{F} \text { for } 24 \mathrm{hr}\right)\end{array}$ & $2.0 \% \max$ & ASTM-D621-64 \\
\hline
\end{tabular}


Only three groups of metallic materials were available at that time that, because of their corrosion resistance, could be considered for the selection of the hatch and bottom insert plate material. The three materials were titanium, stainless steel, and monel. Titanium was the most desirable one as its corrosion resistance was superior to the two other groups of materials. Its compressive strength and tensile strength were higher than for the other two groups of materials, and its specific gravity was the least, easing the opening of the lightweight hatch by the crew from the capsule's interior in an emergency. Unfortunately, the price of titanium forgings was about $500 \%$ higher than for the other materials, and this made its use inapplicable. Thus the choice was between monel and stainless steel, among which only type 316 satisfied the minimum requirement for corrosion resistance. Since the strength of type 316 stainless steel was about equal to that of the monel available, and since the desired shapes of type 316 stainless steel were more readily available from the suppliers, it was selected as the material for fabrication of the hatch and bottom insert plates. The mechanical and physical properties of the type 316 stainless steel are shown in Table 2.

Table 2. Properties of Type 316 Stainless Steel

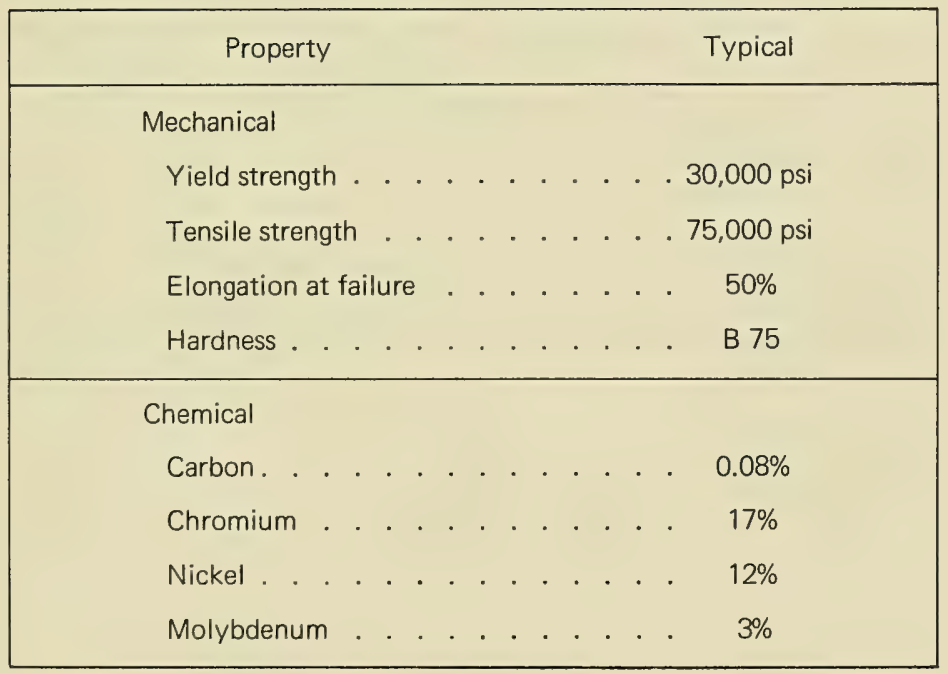


Selection of Capsule Dimensions. The acrylic plastic wall thickness was selected on the basis of (1) elastic theory for stress distribution and (2) elastic instability theory applied to a thick-walled perfect sphere without penetrations. These theories provide only approximations. Acrylic plastic is viscoelastic, rather than elastic and thus basically nonisotropic when stressed; the deformation of the material is not simply a function of stress but also of time and modulus of elasticity, which changes from point to point in the acrylic plastic hull according to the stress distribution.

However; under short-term loading the theory for thick-wall elastic stress distribution gives a good first-order approximation of what the wall thickness of the capsule should be in order to be within a specified compressive stress range. Although this theory does not predict the magnitude of stress raisers around the hatches, it was felt that if the average membrane stress level selected is low enough, the increase in stress generated by the presence of steel hatches will not surpass the ultimate tensile and compressive strength of the acrylic plastic and will not cause ultimate failure of the acrylic plastic material, although some viscoplastic deformation may take place.

The elastic stability calculations are also only approximations. The viscoelastic and viscoplastic time-dependent behavior of the acrylic plastic material negates the basic premises underlying the elastic stability calculations. But as in the calculation of stresses, it was felt that a first-order approximation is acceptable so long as the calculation of implosion pressure was limited to (1) the short-term loading case, in which the effect of time on viscoelastic behavior of material is minimized, and (2) the selected implosion pressure was higher than the operational pressure by some reasonably high safety factor.

Since elastic instability of external pressure hulls for shallow depths is generally the deciding factor in determining wall thickness, ${ }^{16,17,18}$ it was used as the primary design consideration. The classical analytical expression for elastic instability developed by Zoelly in 1915 was originally derived ${ }^{16}$ for perfect spheres.

$$
p_{c r}=\frac{K E\left(\frac{t}{R_{m}}\right)^{2}}{\sqrt{1-\mu^{2}}}
$$

where $\mathrm{p}_{\mathrm{cr}}=$ critical pressure at which elastic instability occurs (psi)

$E$ = Young's modulus (psi)

$\mathrm{t}=$ shell thickness (in.) 


$$
\begin{aligned}
& \mathrm{R}_{\mathrm{m}}=\text { radius to midsurface of shell (in.) } \\
& \mu \quad=\text { Poisson's ratio } \\
& \mathrm{K}=\text { buckling coefficient }
\end{aligned}
$$

Therefore, the buckling coefficient $K=1.15$ derived by Zoelly applied only to small deflections. Because of the impossibility of obtaining perfectly spherical shells, the classical small deflection theory for elastic instability has been found to predict critical pressures that are actually 20 to $300 \%$ higher than experimental collapse pressures of spherical shells. Research ${ }^{16,17,18}$ has shown that the deviation of experimental collapse pressure from the calculated critical pressure for perfect spheres is a function of deviations from nominal sphericity and nominal wall thickness. Therefore, attempts were made to modify the classical formula and to specify tighter dimensional tolerances for the spheres. The resulting semiempirical expression ${ }^{16}$ of

$$
p_{c r}=\frac{0.8 E\left(\frac{t}{R_{0}}\right)^{2}}{\sqrt{1-\mu^{2}}}
$$

where $R_{0}$ is the external radius (in.), has been found to show good agreement with experimental data so long as the deviation in sphericity in shells is less than $0.03 t$ and the minimum wall thickness value is used for the term $t$ in Equation 2. To make this equation applicable to acrylic plastic material, one further modification is necessary. In place of Young's Modulus $E$, based on linear relationship between stress and strain, new terms must be substituted that reflect the nonlinear relationship between stress and strain in acrylic plastic material. The new term for $E$ may be tangent modulus of elasticity, $E_{t}$, secant modulus of elasticity, $E_{s}$, or a hybrid expression of $\sqrt{E_{s} E_{t}}$ that takes both moduli into consideration. The new term for $\mu$ may be $\mu_{v}, a$ variable that also reflects the nonlinear relationship between stress and Poisson's ratio in acrylic plastic.

The secant modulus, tangent modulus, and variable Poisson's ratio are generally derived from typical uniaxial compression stress-strain curves of acrylic plastic test specimens. In order to arrive at the stress level in the sphere so that the proper $E_{s}, E_{t}$, and $\mu_{v}$ can be substituted into Equation 2, the following simplified equation was used

$$
s=\frac{p\left(R_{0}\right)^{2}}{2 R_{m} t}
$$

where s denotes average membrane stress, which for the purposes intended is of sufficient accuracy. 
Thus, the resulting equation for prediction of instability-type failure in acrylic plastic shells can be stated as

$$
P_{c r}=\frac{0.8 \sqrt{E_{s} E_{t}}\left(\frac{t}{R_{o}}\right)^{2}}{\sqrt{1-\mu_{v}^{2}}}
$$

When this formula is utilized, it is imperative that in order for the formula to predict critical pressure of an acrylic plastic sphere the material properties used in the formula must be obtained under stress rates that are identical to that used for implosion of the sphere. In practice, this means that, when the Equation 4 is used to predict the critical pressure of an acrylic plastic sphere under a short-term pressure loading condition (pressurization at some constant rate to implosion), the stressing rate of the material test specimens should be the same, or very close to the stressing rate of the sphere.

Thus as the first step in calculating the thickness of the spherical capsule, a stress-strain relationship had to be established for grade $\mathrm{G}$ Plexiglas under a stressing rate similar to the one to be used in short-term implosion tests of acrylic plastic capsules. Since the stress rate tentatively selected for capsule implosion tests was to be in the 800-to-1,000-psi/min range, similar stressing rates were used to determine the material properties of acrylic plastic (Figures 14a through 14d).

Utilizing the $E_{s}, E_{t}$, and $\mu_{v}$ determined by uniaxial tests and simultaneously solving Equations 3 and 4 , the wall thickness required for implosion by the mechanism of general elastoplastic instability failure was calculated to be $t / R_{o}=0.0685$ and the average membrane stress at critical pressure $s_{\text {avg }}=10,200$ psi. At a critical pressure of 1,350 psi (equivalent to 3,000-foot depth) and an average stress level of 10,200 psi, the mechanical properties of acrylic plastic were determined to be $E_{s}=402,000$ psi, $E_{t}=$ 270,000 psi and $\mu_{v}=0.375$ (Figures 14a through 14d).

Once the $t / R_{o}$ proportion of the sphere was established at 0.0685 , detailed calculation of stresses could take place. Using the analytical expressions for distribution of stresses in a thick-walled sphere of elastic material

$$
\begin{gathered}
S_{1}=S_{2}=-p\left[\frac{R_{o}{ }^{3}\left(R_{i}^{3}+2 r^{3}\right)}{2 r^{3}\left(R_{o}{ }^{3}-R_{i}{ }^{3}\right)}\right] \\
S_{3}=-p\left[\frac{R_{o}{ }^{3}\left(r^{3}-R_{i}{ }^{3}\right)}{r^{3}\left(R_{o}{ }^{3}-R_{i}{ }^{3}\right)}\right]
\end{gathered}
$$


where $\mathrm{S}_{1}=$ meridional membrane stress (psi)

$\mathrm{S}_{2}=$ equatorial membrane stress (psi)

$\mathrm{S}_{3}=$ radial wall stress $(\mathrm{psi})$

$\mathrm{p}=$ external hydrostatic pressure (psi)

$\mathbf{R}_{0}=$ external radius of sphere (in.)

$\mathbf{R}_{\mathbf{i}}=$ internal radius of sphere (in.)

$r=$ radius to point where stress is to be found (in.)

Solving Equations 5 and 6 for $S_{1}$ and $S_{3}$ when $p=450$ psi $(1,000$-foot operational depth), the following stress values were determined for internal and external shell surfaces:

$$
\begin{aligned}
& \text { at } R_{0} S_{1}=S_{2}=-3,330 p s i \text { and } S_{3}=-450 p s i \\
& \text { at } R_{i} \\
& S_{1}=S_{2}=3,550 \mathrm{psi} \text { and } S_{3}=0
\end{aligned}
$$

From consideration of the calculated stresses, it appeared that the selection of the $t / R_{0}=0.685$ wall thickness for the sphere on the basis of the elastoplastic instability Equation 4 is probably satisfactory from the stress magnitude viewpoint also, as the general stress level in acrylic plastic at the operational depth of 1,000 feet appears to be less than 4,000 psi. If the stress magnitude level turned out subsequently to be too high because of the local stress raiser effect of metal plates in the polar penetrations, the reduction in operational depth from 1,000 to 600 feet (minimum continental shelf depth requirement) would probably lower the overall stress level sufficiently to bring the peak stress at the hatch penetration below ultimate failure stress level for acrylic plastic.

Selection of the hatch plate thickness presented a more complicated problem than the selection of acrylic plastic hull thickness. From the viewpoint of stresses in the hatch plate, it was desirable to make the plates as thick as possible, while from the viewpoint of stresses in the acrylic plastic bearing surface, it was mandatory to keep the thickness to the absolute minimum so that the mismatch in wall rigidity was minimized. Since neither the effect of hatch plate thickness on the stress concentration in acrylic plastic, nor the effect of acrylic plastic hull on the magnitude of stresses in the metallic hatch was known for sustained loading during a typical dive, either one of the viewpoints taken would result in an unknown quantity. Ultimately the viewpoint was taken that minimization of stress raiser effect in acrylic plastic was more important than minimizing the stress 
level in the metallic plate. If the stresses in the hatch plate of chosen dimensions were found to be too high, an alloy with higher material properties could be substituted for the material originally chosen. This would result in no changes in hatch design, while increasing thickness of the acrylic plastic hull would require considerable redesign.

To calculate the minimum thickness of the metallic plates, the assumption was made that the metal plate is unaffected by the presence of the acrylic plastic hull bearing upon it. Therefore the stress in the plate could be approximated on the basis of membrane shell theory. Using a design stress of 13,500 psi (approximately $50 \%$ of minimum yield stress in type 316 stainless steel) and the thin shell membrane shell equation

$$
p=\frac{2 t S_{1}}{R_{0}}
$$

a hatch thickness of $t / R_{0}=0.0166$ was calculated for $p=450$ psi. When the calculated hatch plate thickness was subsequently checked for its elastic stability with Equation 4, it was found to be elastically stable at operational depth. Its failure was predicted on the basis of Equation 4 to take place by plastic instability at pressures slightly in excess of 1,350 psi. Thus from the viewpoint of instability failure, the design would appear to be adequate as it would cause the hatch to fail at approximately the same depth as the rest of the acrylic plastic sphere. To forestall generation of excessive stress raisers around penetrations in the bottom metal plate, they were either reinforced by local thickening of the plate in the form of bosses or flanges, or they were subsequently filled by penetrators of approximately the same rigidity as the plate. Detailed stress analysis of the metal plates was not performed at that time as it was felt there was not sufficient information on the interaction between steel plate and acrylic plastic hull deforming viscoelastically or viscoplastically to make the analytical stress analysis more than a first order approximation.

Structural Component Dimensioning. This constituted the last step in the detail design of the acrylic plastic capsule. Before the detail design of the structural components could be initiated, a procedural question had to be resolved: Which acrylic plastic sphere size would be ultimately selected for the capsule program? Although some capsule dimensions have been calculated in nondimensional terms like $t / R_{0}$, and therefore apply to any size sphere, most dimensions of the capsule details must be based on dimensional human factors criteria (size of hatch, hatch locking mechanism, and others). Thus, once the decision was made to design a certain diameter capsule, a considerable waste of engineering and design time would occur if the diameter of the capsule was changed. 


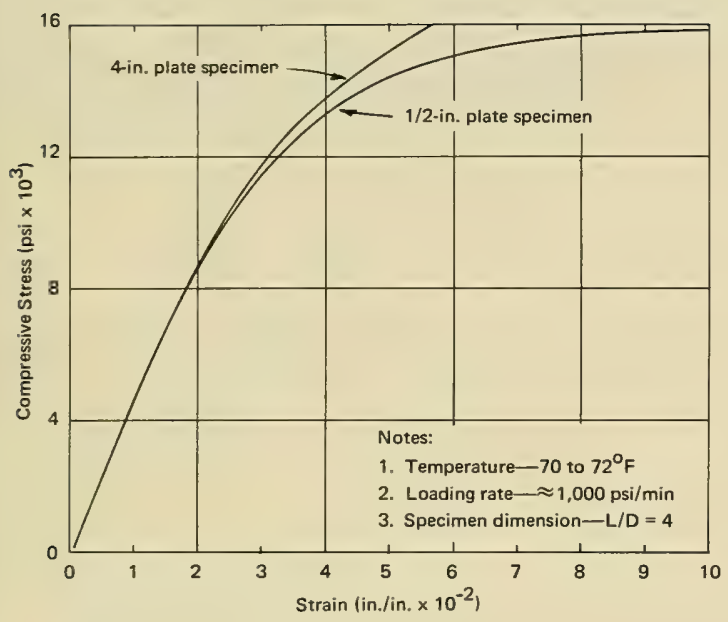

Figure 14a. Strain-versus-stress relationship for grade G Plexiglas acrylic plastic under uniaxial compression.

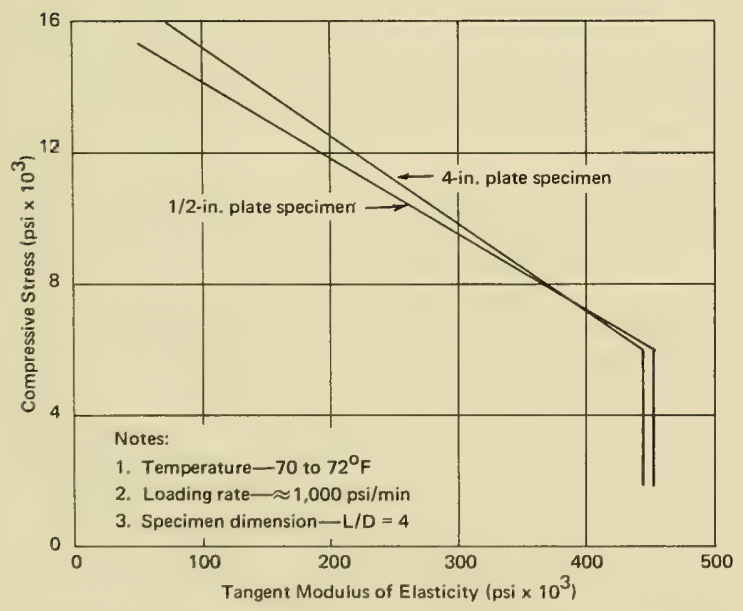

Figure 14c. Tangent modulus of elasticity versus stress for grade $G$ Plexiglas acrylic plastic under uniaxial compression.

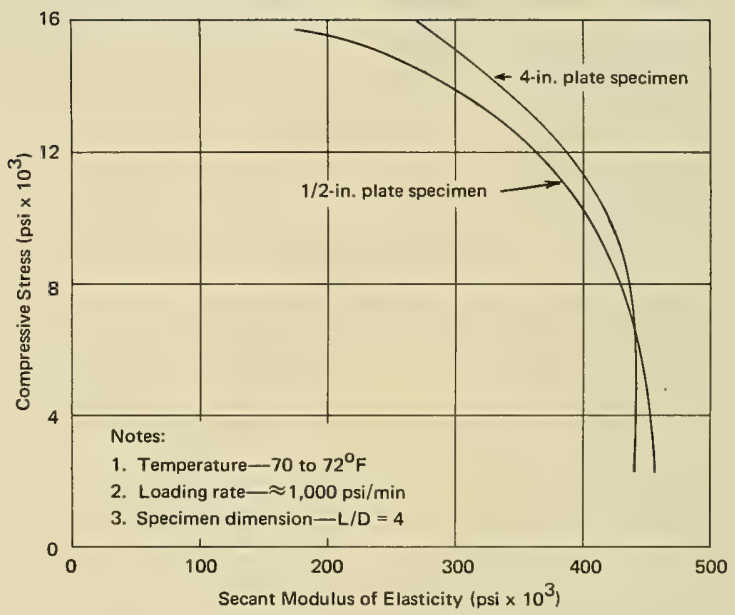

Figure 14b. Secant modulus of elasticity for grade G Plexiglas acrylic plastic under uniaxial compression.

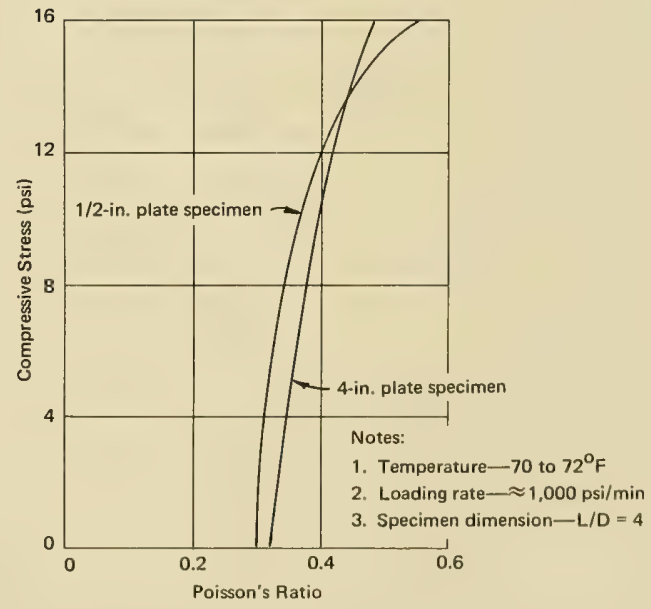

Figure 14d. Poisson's ratio versus stress for grade G Plexiglas acrylic plastic under uniaxial compression. 

The diameter chosen at that time for the operationally most useful panoramic capsule concept was 120 inches. That diameter was arrived at on the basis of two considerations: (1) that this is the minimum size that will permit a two-man crew to remain comfortably on the ocean bottom submerged longer than a day, and (2) that this is the largest capsule for continental shelf depth that can be built around the required $t / R_{0}=0.0685$ from 4.125 inch thick material, the thickest commercially available acrylic plastic plate. Since during the course of the acrylic plastic capsule program a smaller prototype operational capsule of 66-inch diameter was to be built, the operators would have to put up with scaled-down hatches, penetrations, attachments, etc., of less than ideal proportions from the human factors viewpoint. To permit as many subsequent changes in the capsule design as possible, only as many details were to be incorporated in the first capsule design as were needed to permit the construction of a 15-inch model for experimental validation of the capsule design. To save time in drafting and detail design, it was decided to bypass one step generally required between the formulation of the full-scale concept and the fabrication drawings for a scale model needed for experimental validation of the full-scale concept. The step bypassed was the detailed design drawing of the full-scale concept, which when scaled down and simplified would have become the fabrication drawing for the small-scale (15-inch) model. Thus, the fabrication drawings for the small-scale model of the capsule were prepared directly from the rough concept sketches. This shortcut made it possible to complete fabrication drawings of the small-scale capsule model in less than 2 months after the formulation of the concept. Because of the elimination of this step in arriving at small-scale model dimensions, the fabrication drawings for any subsequent capsules with diameters larger than 15 inches became only scaled-up versions of the 15-inch model capsule. There are some shortcomings associated with this approach. However, it was felt that the advantage of being able to check experimentally the quite radical concept before considerable time and money were expended on engineering the details of the concept offset the major shortcoming: freezing the design of the capsule during the first 2 or 3 months of the acrylic plastic capsule program before a thorough engineering study could be undertaken on the many feasible design trade-offs.

The resulting fabrication drawings (Figures 15 through 23) of the capsule model reflect quite faithfully the previously discussed conceptual approaches to (1) means of ingress and egress, (2) penetrations for electric and hydraulic circuits, (3) attachment of the capsule to other NEMO structural systems, (4) magnitude of working stresses, (5) specification of construction materials, and (6) hull thickness. No attempt was made to 
include life-support equipment, railing, ladders, or any other structural components that have little influence on the stress distribution in the acrylic plastic capsule. It is well to point out, however, that the actual $t / R_{0}$ ratio of the 15-inch models is somewhat smaller than that calculated previously in the section "Selection of Capsule Dimensions." There was only one reason for the $t / R_{0}$ ratio of the 15 -inch models to be in the 0.060 to 0.0685 range rather than at exactly 0.0685 as calculated: variation in thickness of commercial acrylic plastic plate from the nominal 0.5-inch plate thickness. Procurement of a nominal 0.625 -inch-thick plate that subsequently could be ground to the specified 0.513 -inch thickness would have produced models with the exact $t / R_{0}=0.0685$ ratio, but only at the expense of $300 \%$ increase in the fabrication cost of each capsule model.

Although at a first glance the use of models with a somewhat thinner wall than calculated appeared to be quite unacceptable, there was a positive gain: the experimental data generated by destructive testing of models under short-term, long-term, or cyclic pressure loading would be conservative.

\section{PHASE 2-FABRICATION PROCESS}

The selection of the proper fabrication process posed considerable difficulties for two reasons: (1) it had to perform equally well in the fabrication of large-scale and model capsules, and (2) economical fabrication techniques for model capsules generally turn out to be uneconomical for large-scale capsules, and vice versa. For reasons already briefly discussed, the fabrication of the acrylic plastic capsule was chosen to conform closely to Professor Piccard's concept except for the introduction of material grades and shapes not commonly available in his day.

\section{Acrylic Hull Fabrication}

Material Selection. The material selected for the fabrication of both model capsules and any future full-scale capsules was grade G Plexiglas acrylic plate stock. This material is the commercial grade acrylic plastic widely available as an off-the-shelf plate and sheet stock item in many different sizes and thicknesses.

Although the acrylic plastic under the trade name of grade G Plexiglas was chosen for the NEMO program because of the proximity of a major supplier that carries this material in stock, other commercially available acrylic plastic plates with equivalent material properties sold under other trademarks (Swedlow 310, Acrylite, and others) probably could have been used with about the same success. 

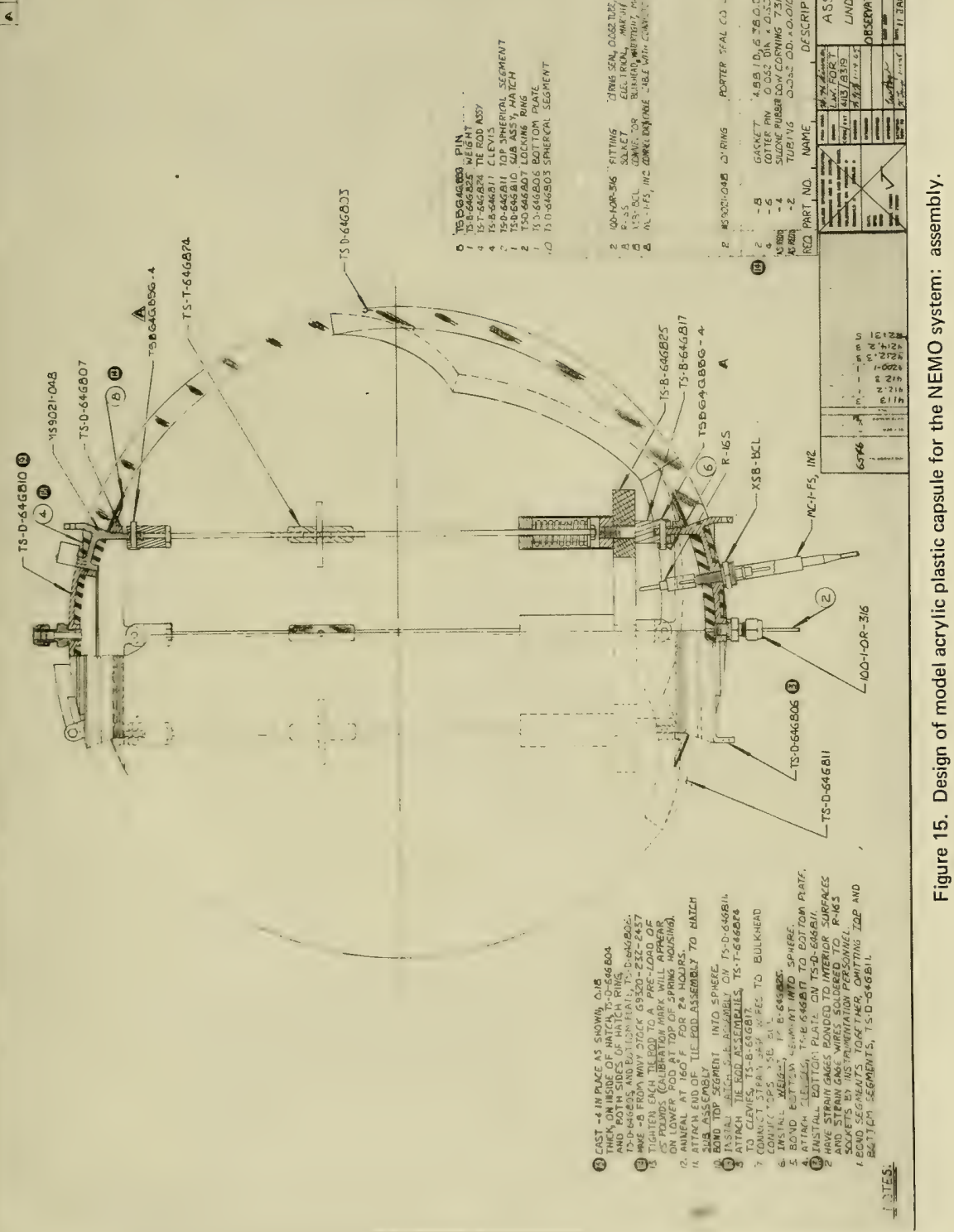


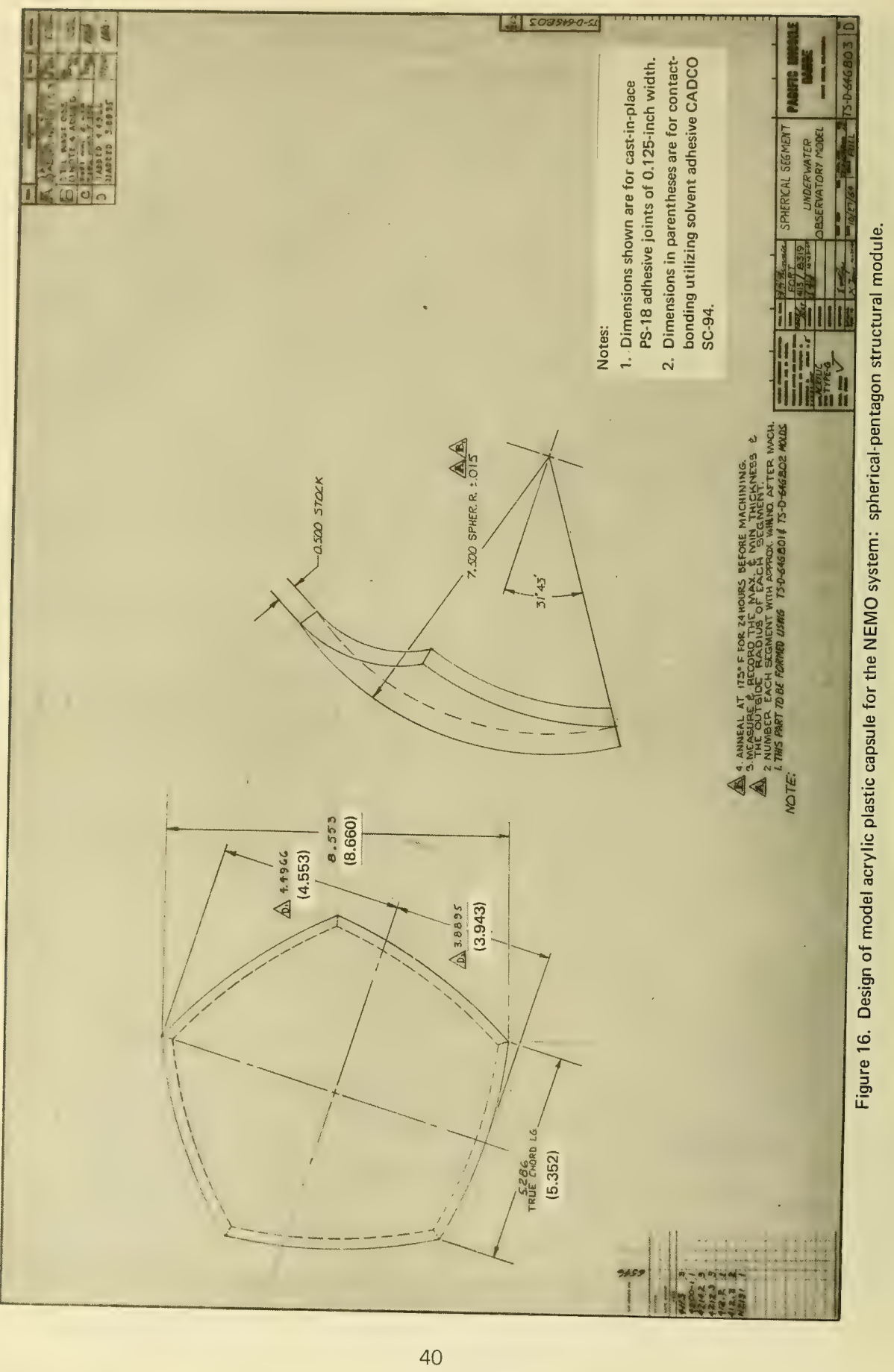




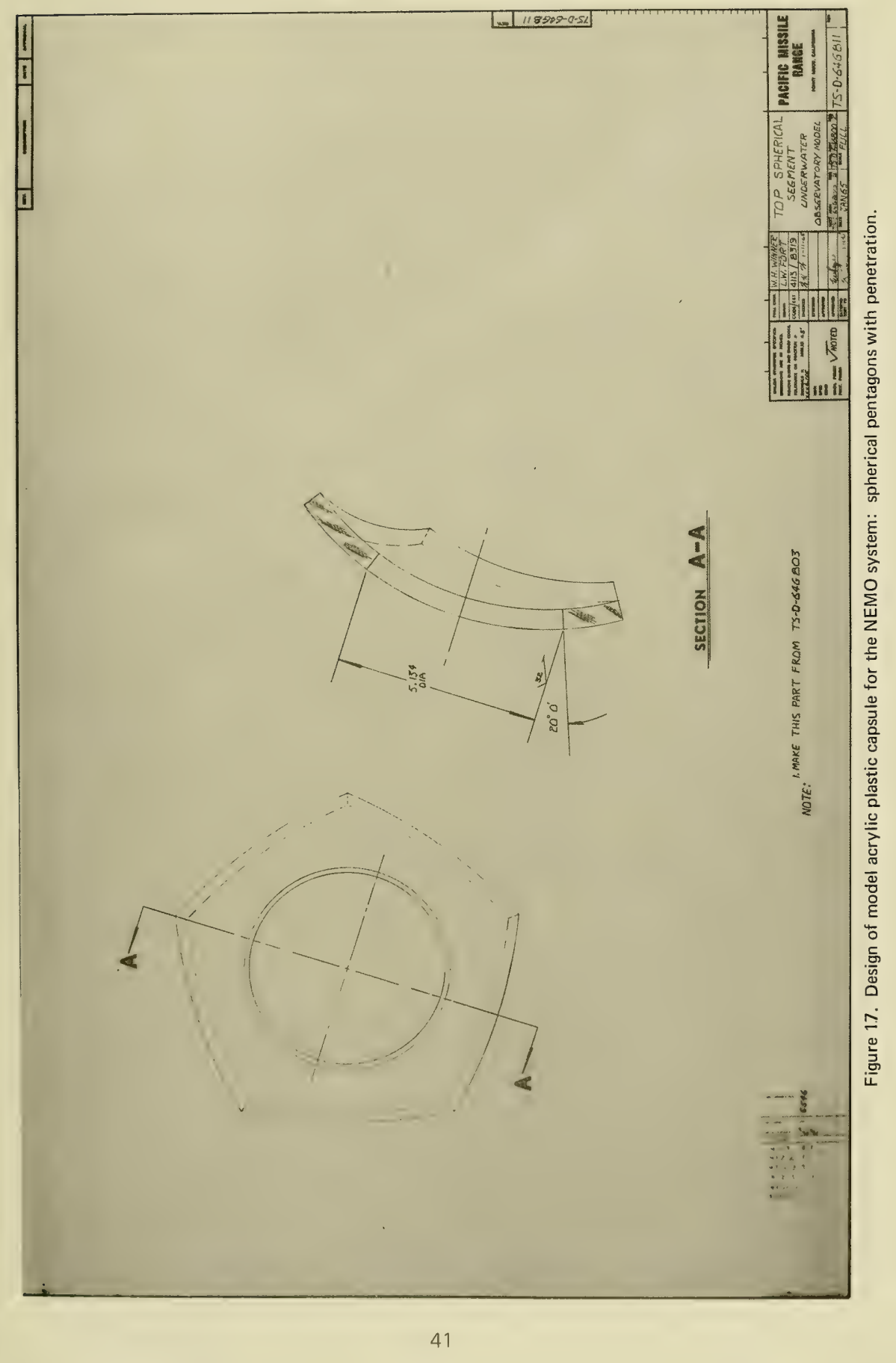




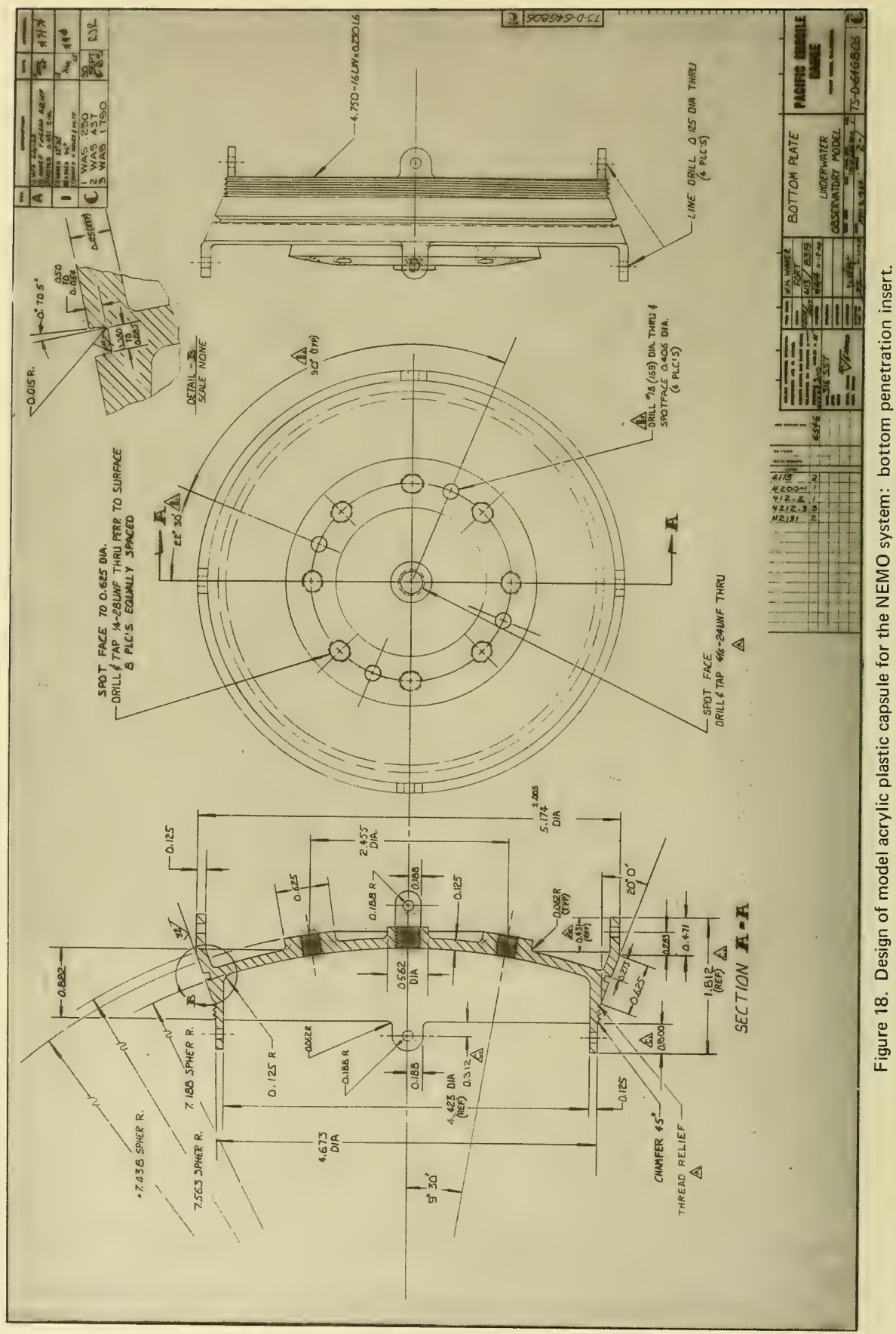




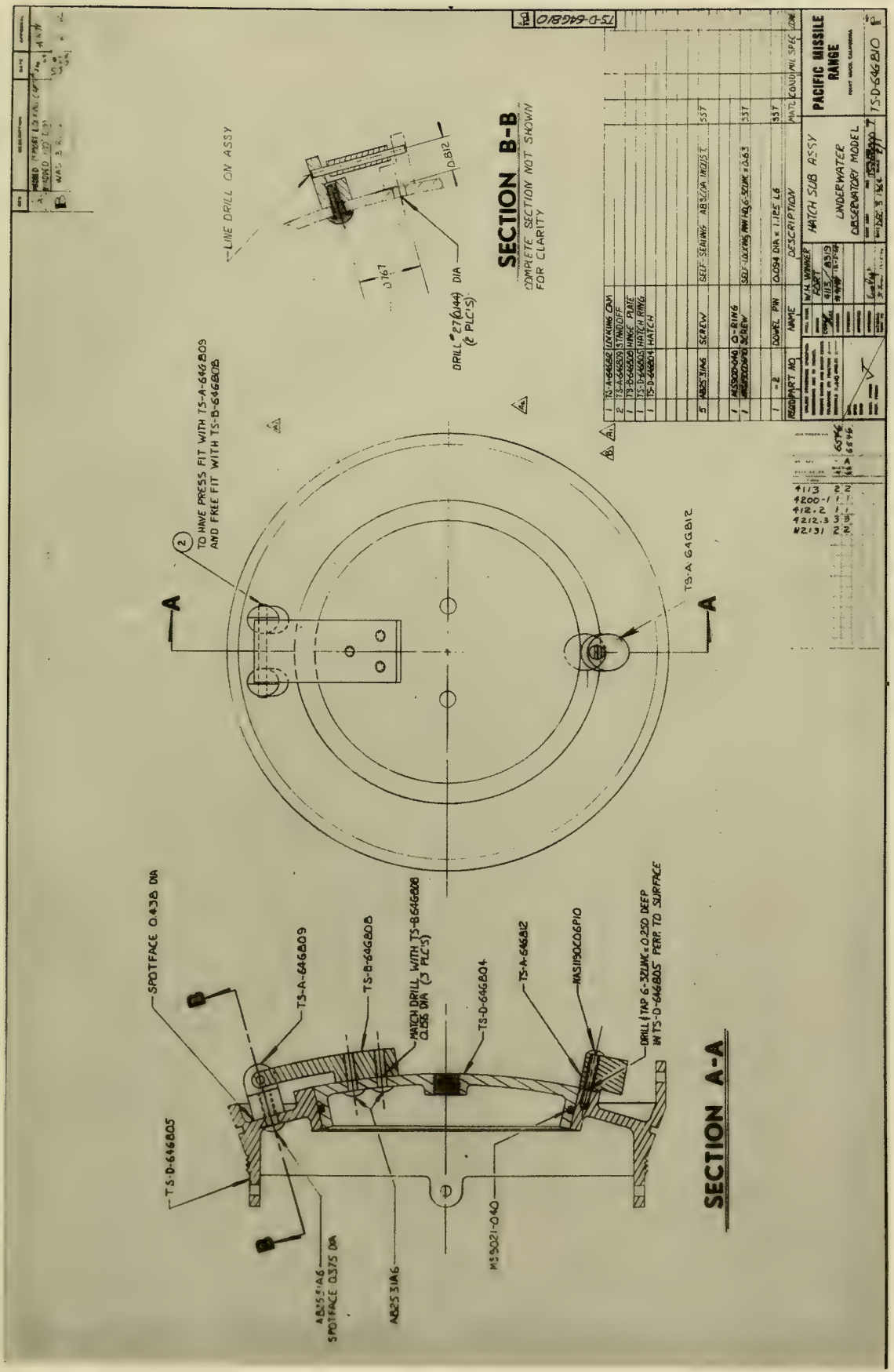

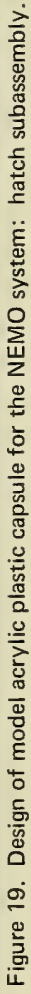




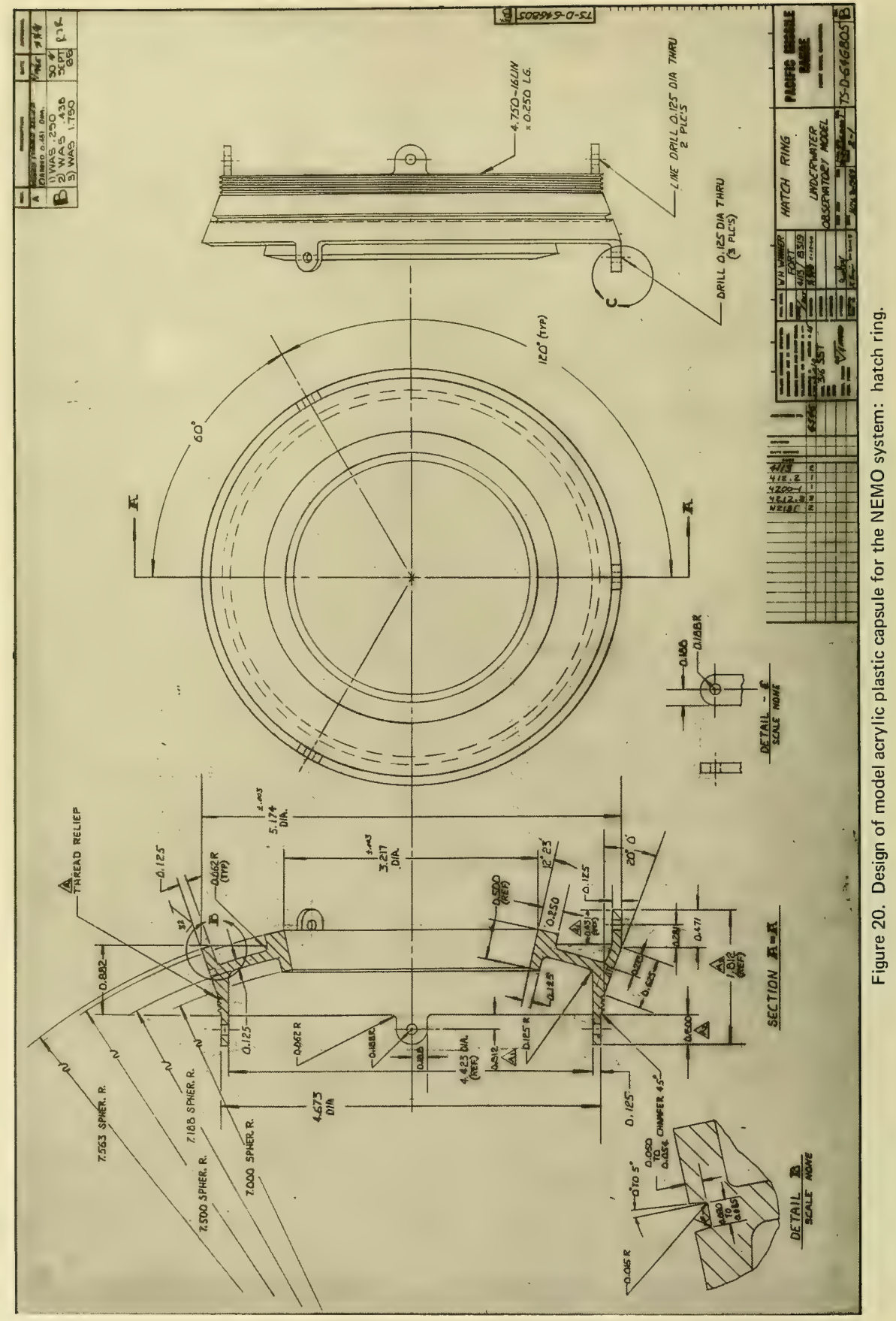




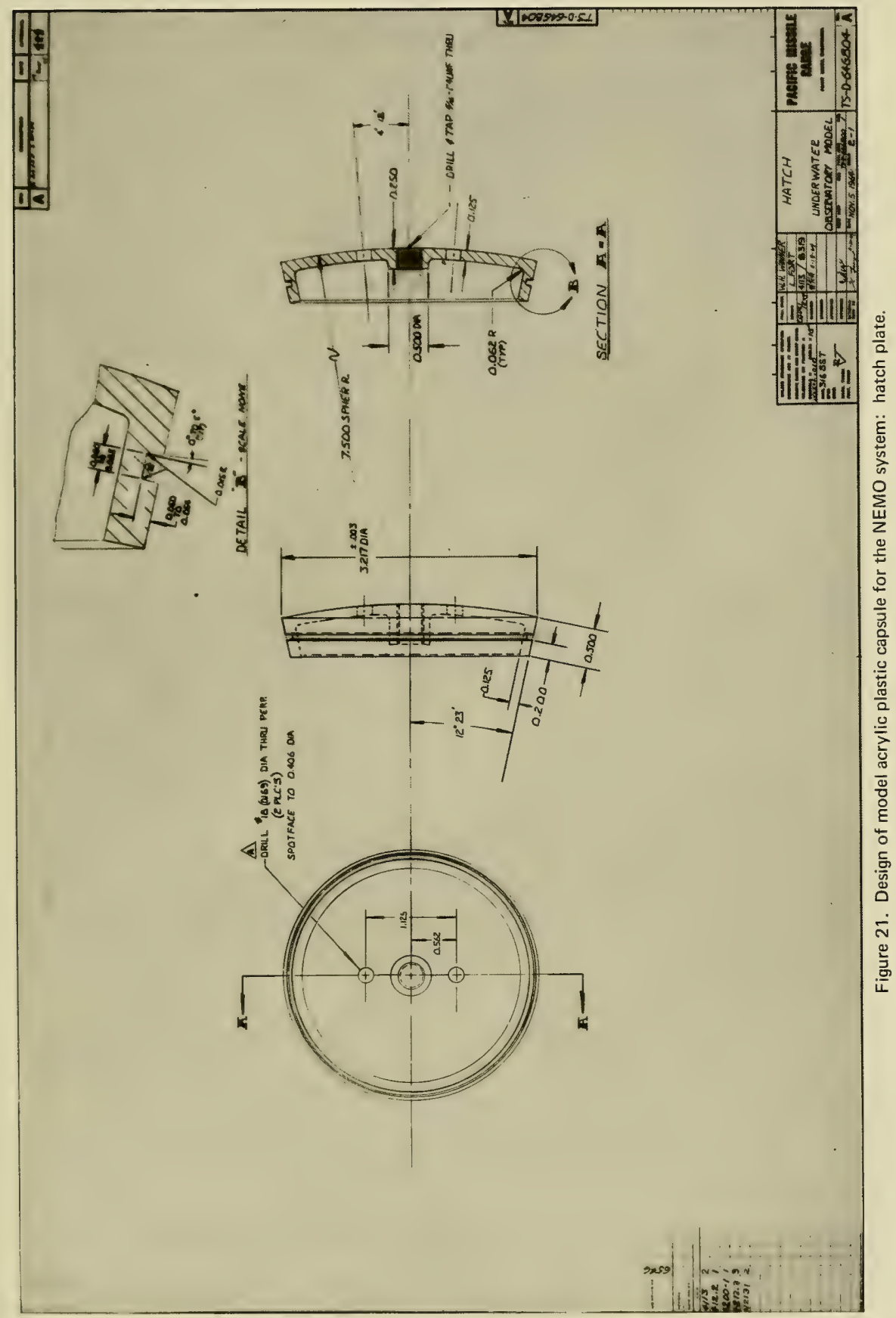




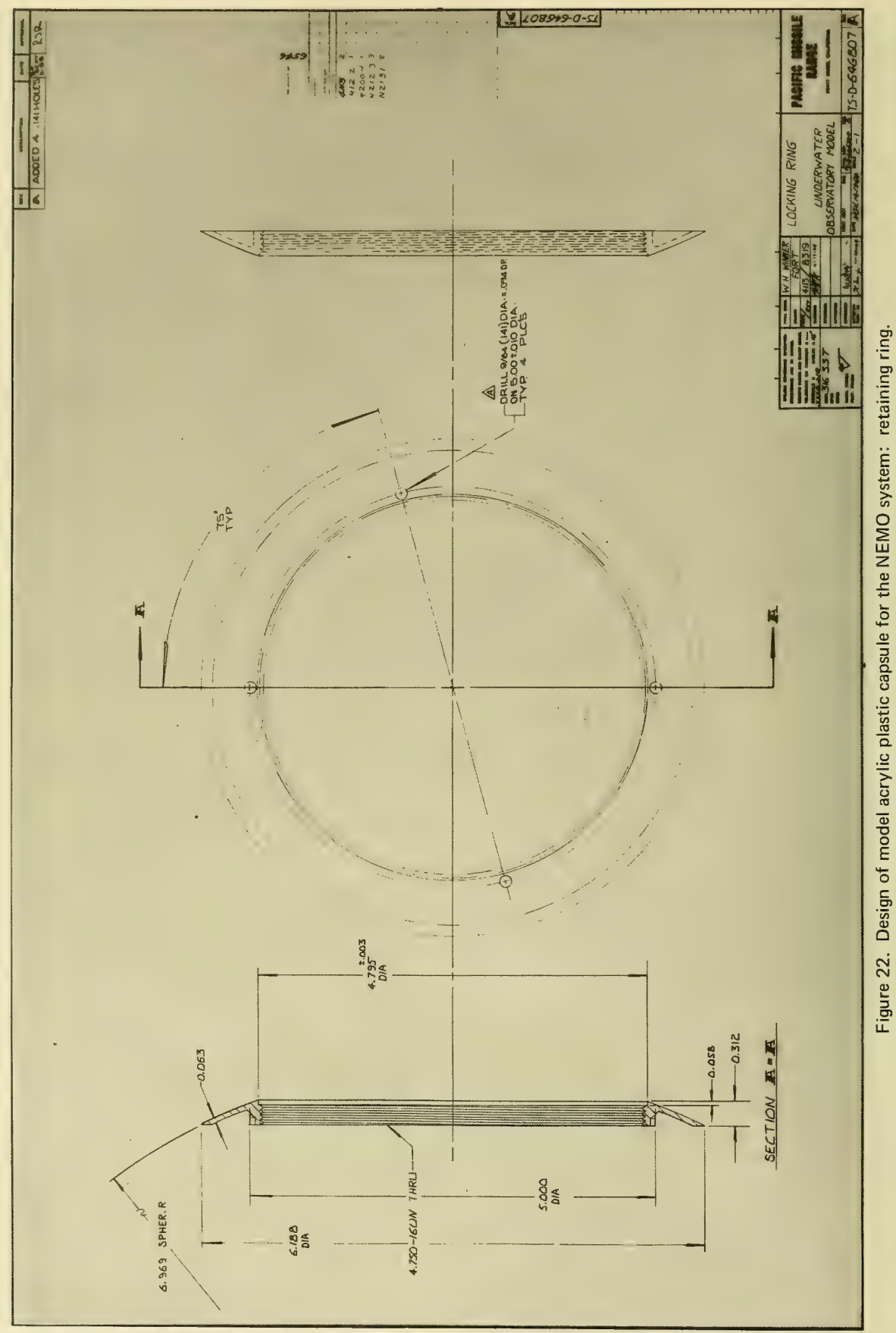




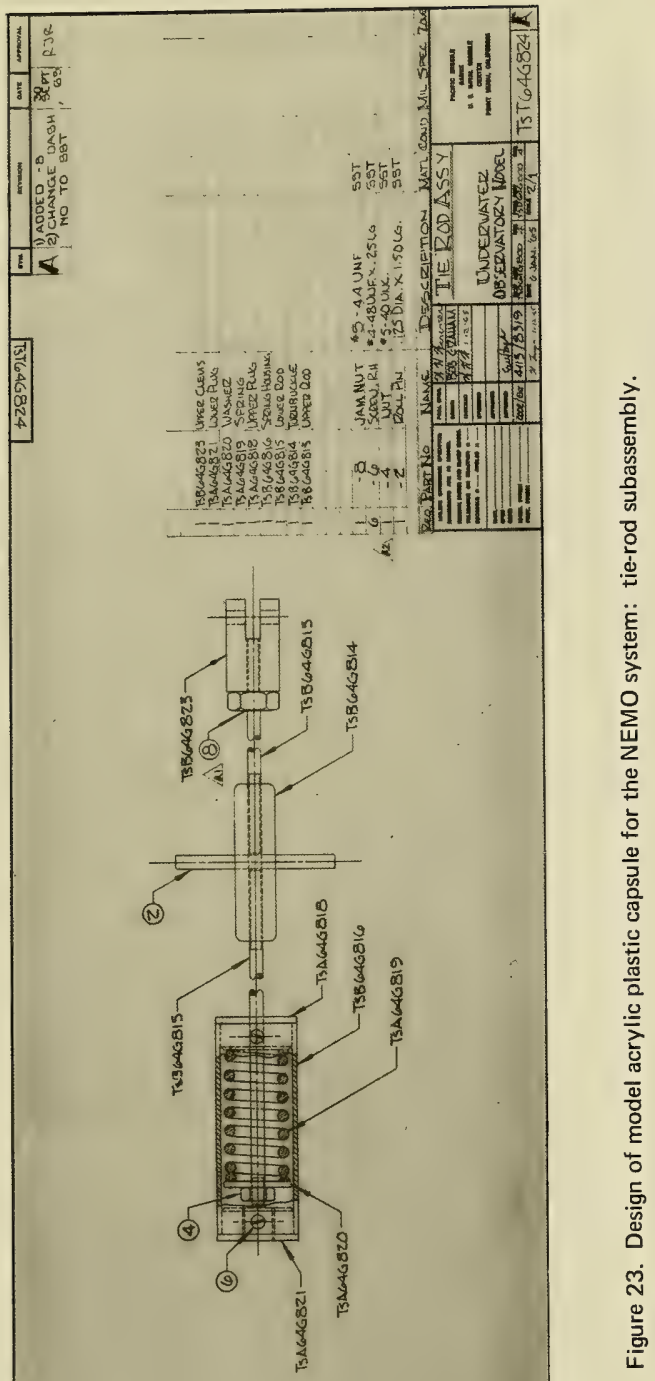


No effort was made to utilize premium grade acrylic plastic materials, or acrylic plastic materials that have been chemically or physically modified (stretched acrylic for example). It was felt that in the exploratory phase of the program having as its objective the utilization of acrylic plastic as structural material for the hull of a submersible the most common available material should be utilized. Only after the exploratory phase of the acrylic plastic pressure hull program was successfully completed could other acrylic plastic materials be considered that, because of their special material properties, would improve either the fabrication process or the performance of acrylic plastic hulls.

The minimum properties of the commercial grade acrylic plastic plate stock used in the program are shown in Table 1. Since deviations from the material properties published by the material supplier could be expected, extensive tests were conducted on samples cut from plates that were subsequently used in the fabrication of model and large-scale capsules. No significant deviations were found between the material properties advertised by the manufacturer and the properties determined experimentally from test specimens, except for long-term compression creep (ASTM D621-51 at 4,000 -psi load), which was found to be $2 \%$ in some of the 2.5 -inch-thick plates instead of the advertised $0.5 \%$. Plates with exceptionally high (larger than $2 \%$ ) long-term compression creep were not utilized for the construction of the acrylic plastic capsules. No significant differences were found between the material properties of nominal 0.5 -inch-thick acrylic plastic plates used in the fabrication of models and the 2.5-inch plate used in the fabrication of the 66-inch NEMO prototype. The data generated (Figures 14a through $14 \mathrm{~d}$ ) in the previously discussed testing of 0.5 -inch and 2.5-inch acrylic plastic plates were used subsequently in the design of acrylic plastic hulls.

Forming. One of the major problems that had to be resolved successfully before spherical acrylic plastic capsules could be built from the pentagon-shaped structural modules of acrylic plastic plate was how to impart to the commercially available flat plates the desired curvature within tight dimensional tolerances. There were several different forming processes known that could be applied.

The flat acrylic plastic plates could be (1) free-blown to desired radius, (2) blown into a spherical mold, (3) sagged into a spherically concave mold, (4) sagged over a spherically convex mold, (5) vacuum sucked into a concave mold, or (6) pressed into a concave mold by a convex male mold. Each of those forming processes possessed advantages and disadvantages that had to be carefully evaluated to arrive at the forming process best suited for the forming of structural hull modules. 
A thorough evaluation indicated that free-blowing flat plates into spherical modules would result in the largest variation in spherical radius from one module to another, and pressing the flat plates between male and female molds of appropriate radii would result in the least variation. In addition, the free-blowing process produces the largest variation in thickness between different points on the module, while the pressing process produced not only a uniform thickness from point to point on a module, but also from one module to another. Thus, it was obvious that the forming process by pressing was the most applicable to fabrication of a spherical acrylic plastic capsule, in which undue deviations from sphericity and uniformity in thickness would exert a deleterious effect on its implosion pressure.

On the basis of this selection process, pressing dies were designed for the fabrication of 15-inch models and the 66-inch operational prototype (see Appendix A for detailed dimensions of the 66-inch capsule). However, only the dies for the model were built initially as it was felt that some experience in using them was required before the decision could be made to invest a considerable sum of money for fabrication of the dies for the prototype 66 -inch acrylic plastic sphere. As soon as the press die assembly was built, it was placed in operation for molding structural modules for the 15-inch capsules.

Model structural modules were formed in a female die with a 7.5-inch concave radius and a male die with a 7 -inch convex radius (Figure 24). The female die had holes in its bottom through which vacuum could be applied to the space between male and female dies. Both dies were placed in a vertical hydraulic press that applied sufficient force to bring the male die to its bottom position 0.5 inch above the female even if the acrylic plastic was several thousands of an inch thicker than 0.500 . The alignment between the male and female dies was assured by rigid mounting in the press platens and by steel guide pins on the male die that fitted holes in the female die. Bottom stops were provided on the guide pins to assure that the male die would not move any closer to the female die than 0.5 inch even if the acrylic plastic blank was under 0.5 inch in thickness. Finish on the die surfaces contacting the acrylic plastic was $32 \mathrm{rms}$.

The first step in the forming process was bandsawing and subsequently turning in the lathe a 9.875-inch-diameter disc from nominal 0.5-inch grade $\mathrm{G}$ Plexiglas acrylic plastic stock. Subsequently, it was stripped of protective paper covering and was placed into an oven whose operational temperature was adjustable. After heating the acrylic plastic to the forming temperature (discussed below) at a rate of approximately $15^{\circ} \mathrm{F} /$ minute, it was removed from the oven and placed into the open die assembly which was preheated by an electric heater in the press platens to $160^{\circ} \mathrm{F}$. Vacuum was immediately applied to the female die followed by closing of the die assembly with the 
hydraulic press. After 3 minutes, the male die was raised while the application of vacuum continued. When the temperature of the formed acrylic plastic decreased to $160^{\circ} \mathrm{F}$ after about 12 minutes, the acrylic plastic was removed from the female mold and cooled on a workbench.

Although the supplier of acrylic plastic plate recommended heating the acrylic plastic to $340^{\circ} \mathrm{F}$ and the dies to $170^{\circ} \mathrm{F}$ prior to forming, some exploratory experiments were conducted to verify those settings. Experimentation indicated that although the magnitude of residual stresses in acrylic plastic after forming is inversely proportional to the temperature of the molds and of the acrylic plastic, the number and sizes of surface blisters and pits is directly proportional to the magnitude of those two temperatures. By varying the temperature of the acrylic plastic and of the dies, it was found that if the acrylic plastic was preheated to 310 to $320^{\circ} \mathrm{F}$ and subsequently placed into $160^{\circ} \mathrm{F}$ dies, the number and severity of surface blisters was not noticeable, while at the same time the residual stresses were not too excessive. Lowering the temperature of acrylic plastic to $260^{\circ} \mathrm{F}$ eliminated all surface bubbles and blisters, but introduced stresses of unacceptable magnitude.

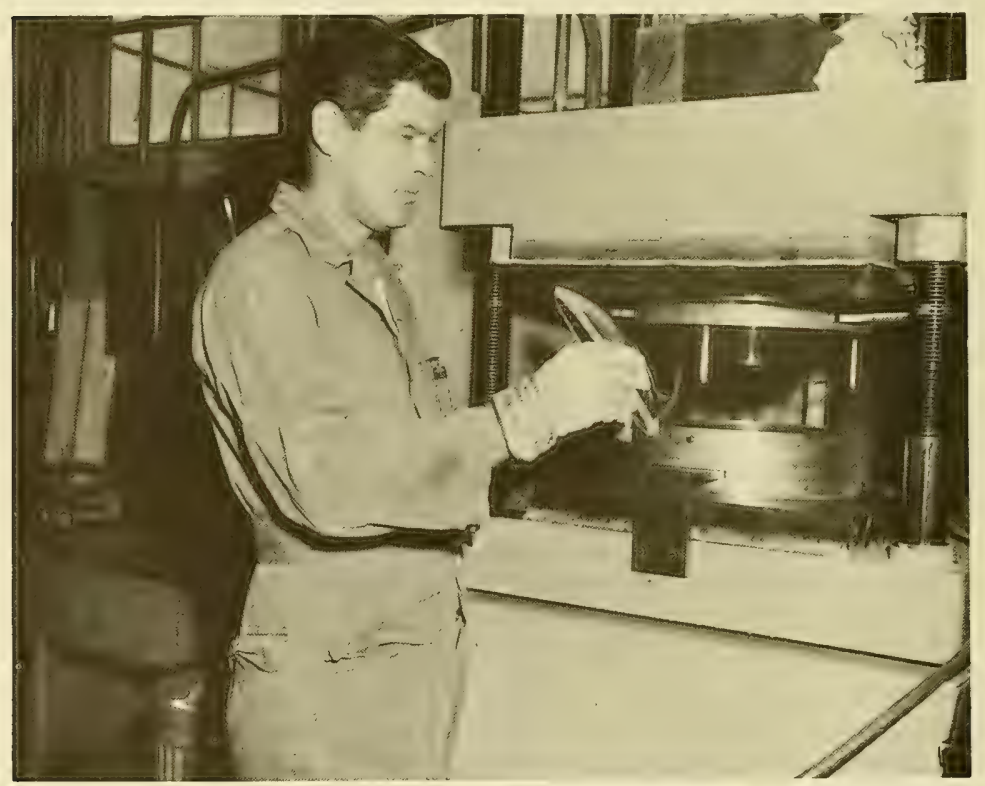

Figure 24. Hydraulic press with matched male and female die assembly for pressing spherical sectors for model capsules. 
At the $310^{\circ} \mathrm{F}$ temperature of acrylic plastic and $160^{\circ} \mathrm{F}$ temperature of the dies, the residual maximum shear stress at the edge of the dished disc was measured photoelastically (see Appendix B for discussion of photoelastic effects in acrylic plastic) to be approximately 3,700 psi. The magnitude of residual stresses decreased somewhat towards the center of the formed disc. Thus, for example, the maximum shear stress 1.5 inches away from the edge of the disc was only 1,500 psi.

An attempt was made to eliminate the residual stresses by subsequent annealing of the formed spherical sector. The formed sectors were placed on a flat surface and inserted in a 170 to $180^{\circ} \mathrm{F}$ oven for 24 hours. After the sector had cooled to $95^{\circ} \mathrm{F}$ at a rate of approximately $7^{\circ} \mathrm{F} /$ hour, it was checked dimensionally and photoelastically. The dimensional check disclosed that the radius of curvature increased by approximately 0.020 inch while the magnitude of residual stresses decreased only very slightly. The basic change in residual stresses consisted of lowering the peak stresses by more evenly redistributing the residual stresses over the whole spherical sector surface. Because of the dimensional change generated in spherical sectors during annealing, it was decided in the future to anneal the spherical sectors only after they had been machined into pentagons and bonded into a sphere, as then they would be constrained by the spherical shape of the hull from changing their spherical radii excessively. An alternative approach was considered in which the spherical segments would be placed into a mold for annealing, so that the mold would give the sector even support and thus prevent changes in curvature due to sagging. Although this approach was considered feasible, it was not utilized as it was felt that the mold and the oven would be involved too long while individually annealing the 300 spherical sectors needed for fabrication of 25 acrylic plastic capsule models. However, individual annealing of spherical sectors was recommended for fabrication of the single 66-inch prototype capsule, as the individual annealing of only 12 sectors would not impose an undue time requirement on the available oven and mold.

The change in thickness of the acrylic plastic plate after thermoforming, but prior to annealing was measured. The thickness of the acrylic plastic disc, for example, changed from the previously uniform 0.494 inch to 0.485 inch in the center, to 0.480 inch along the edge ( $1 / 2$ inch from the edge), and to about 0.470 to 0.475 midway between the center and the edge (Figure 25). These small changes in thickness were considered to be reasonable as the absolute change in thickness was actually less than $5 \%$, and uniformity of thickness change within $3 \%$. 
Flat Blank

Material = grade G Plexiglas, unshrunk, nominal 0.5-in. plate

Flat blank diameter $=9-7 / 8$ in .
Circumference of flat part $=31.0 \mathrm{in}$.

Actual thickness $=$ uniform, 0.494 in.

Area of flat blank $=76.3$ in. ${ }^{2}$

\section{Formed Spherical Sector}

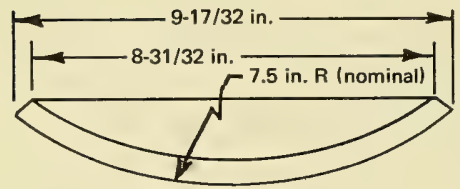

Cross Section at Centerline
Circumference of 8-31/32-in. chord $=28.17$ in. Circumference of 9-17/32-in. chord $=29.95$ in. Length of the 7-in. $-R$ arc $=9.7 \mathrm{in}$.

Length of the 7.5-in. $-R$ arc $=10.25 \mathrm{in}$.

Area of concave side $=71.5 \mathrm{in}^{2}$

Area of convex side $=77.8$ in. $^{2}$

Variation in Thickness of the Formed Part

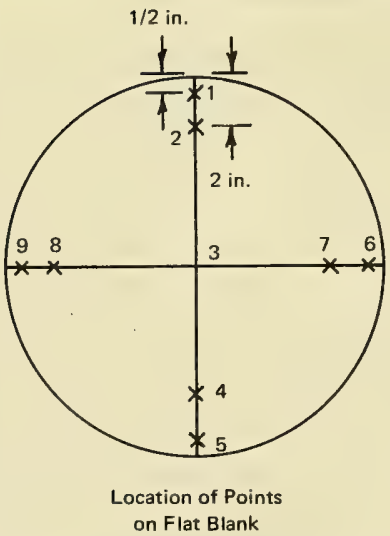

Point No. Thickness (in.)

0.481

0.478

0.485

0.477

0.480

0.480

0.473

0.473

0.477

Figure 25. Typical sphericity and thickness of spherical sectors after die pressing to 7.5 -inch nominal radius of curvature.

In addition to thickness, the curvature of the spherical sector was also measured prior to annealing. In general, the curvature of the spherical sector was uniform and closely matched that of the female die. The external radius of the sector was within 0.000 to 0.005 inch of the mold curvature. The largest deviations occurred at the center and at the edge of the sector, while midway between the center and the edge there was the least deviation 
in curvature. The measurement of the spherical radius on the thermoformed sector concluded the evaluation of the thermoforming process for fabrication of 15-inch model acrylic plastic hulls. Once the selected forming process was found to be feasible for forming the structural modules of the acrylic plastic capsule models, the feasibility of forming structural modules for the 66-inch prototype acrylic plastic capsule was explored.

The method of forming large-scale pentagon-shaped structural modules was similar, but not identical, to the method used for model structural modules. The reason for not following the forming technique developed for the scale models was the high cost of equipment (dies and presses) that this technique would require for forming structural modules for the prototype 66-inch acrylic plastic capsule. To decrease the costs of forming, it was decided to eliminate the male die, and thus also the requirement for a very expensive press. Although it was surmised that the elimination of the press-actuated male die would increase the deviations from desired sphericity and thickness, it was hoped that the increased deviation in sphericity from a nominal 33-inchradius would not be of such magnitude as to make the pentagon-shaped spherical sectors unacceptable for pressure hull construction.

Thus a single female die, henceforth referred to as the mold, was designed and fabricated from aluminum. There was an important departure from the design philosophy used in designing the dies for the 15-inch model. In the previous case, the concave spherical radius of the female die was exactly equal to the nominal external radius of the hull. The female mold to be used in forming sectors for the 66-inch capsule had, however, a concave spherical radius 0.187 inch larger than the nominal external radius of 33 inches. This intentional discrepancy between the nominal sector and mold radii was based on the opinion of fabricating personnel that, because of the elimination of the hydraulic-press-actuated male die, the formed sector would not contact the whole surface of the mold, but just at the apex and at the edges. Thus, in their opinion, if a 33-inch-radius mold was used the curvature of the formed sector would be less than 33 inches in most places on the sector. On the other hand, if a 33.187-inch-radius mold was used, the curvature of the formed modules would be about 33 inches. In absence of convincing arguments to the contrary, it was decided to try this approach.

The thermoforming blank was prepared in the same fashion as were those for the 15-inch models. A circular 46.6 inch blank was first sawed on a bandsaw from a $48 \times 60 \times 2.5$-inch plate of acrylic plastic (Figure 26) and then turned to a 46-inch disc with 200-rms edge finish in a vertical boring mill (Figure 27). One corner of the periphery was chamfered $1 / 8$ of an inch for better support and sealing of the blank in the mold prior to forming. 
During the bandsawing and milling operations, the masking paper was left on to protect the acrylic plastic against scratches. When the machining was completed, the masking paper was removed and the blank placed (chamfered edge downward) into the mold with the aid of a hoist equipped for this purpose with a vacuum-actuated suction disc. Four 0.125 -inch-diameter holes were drilled 2 inches deep into the edge of the disc for placement of thermoelectric couples. After placement of thermoelectric couples, the mold and blank assembly were moved into a walk-in oven and the temperature control set at $165^{\circ} \mathrm{F}$. After approximately 16 hours, the temperature control was set for $310^{\circ} \mathrm{F}$. In approximately 8 hours, thermoelectric couples in the acrylic plastic indicated that a minimum temperature of $300^{\circ} \mathrm{F}$ was reached. At that time, a vacuum of 25 inches of mercury was applied to the mold. The blank sagged immediately and completely. After 15 minutes, the oven was turned off, but the vacuum was left on and the door closed to permit slow cooling of the acrylic plastic to $110^{\circ} \mathrm{F}$. It took 16 hours for the temperature in the acrylic plastic to drop to $110^{\circ} \mathrm{F}$. At that time, the doors to the oven were opened and the formed sector and the mold removed (Figure 28).

Since little was known about the formability of such thick acrylic plastic blanks in a vacuum mold, a careful postforming inspection was performed on the first molded acrylic plastic sector for the 66-inch capsule. The inspection consisted not only of accurately measuring the thickness of the formed sector, but also the sphericity of its concave surface. The sphericity of the concave mold was also measured, as without knowledge of deviations in its sphericity, few conclusions could be drawn on the causes of asphericity in the formed acrylic plastic sector.

The results of measuring the sphericity of the mold (Figure 29), as well as the sphericity and thickness of the formed acrylic plastic sector, are quite interesting (Figure 30). First, the sphericity of the mold was found to deviate at some points on the mold as much as 0.040 inch from the specified 33.000-inch radius of the formal sector, with the least deviation measured at a distance of 12.000 inches from the center of the mold as would be expected from a sphericity-measuring technique that used the surface of the mold at that location as reference datum. Second, the deviations from the nominal sphericity of the formed spherical sector followed closely the trend of the deviations of the surface mold. But since the sphericity of the sector was measured on its concave surface, and the thickness of the sector varied somewhat from point to point (Figure 30), a quantitative comparison between the sphericity of the convex surface on the sector and the sphericity of the concave surface on the mold could not be made accurately. Third, the radius of curvature on the concave surface of the sector appeared to be greater than the 30.500 inches specified. 


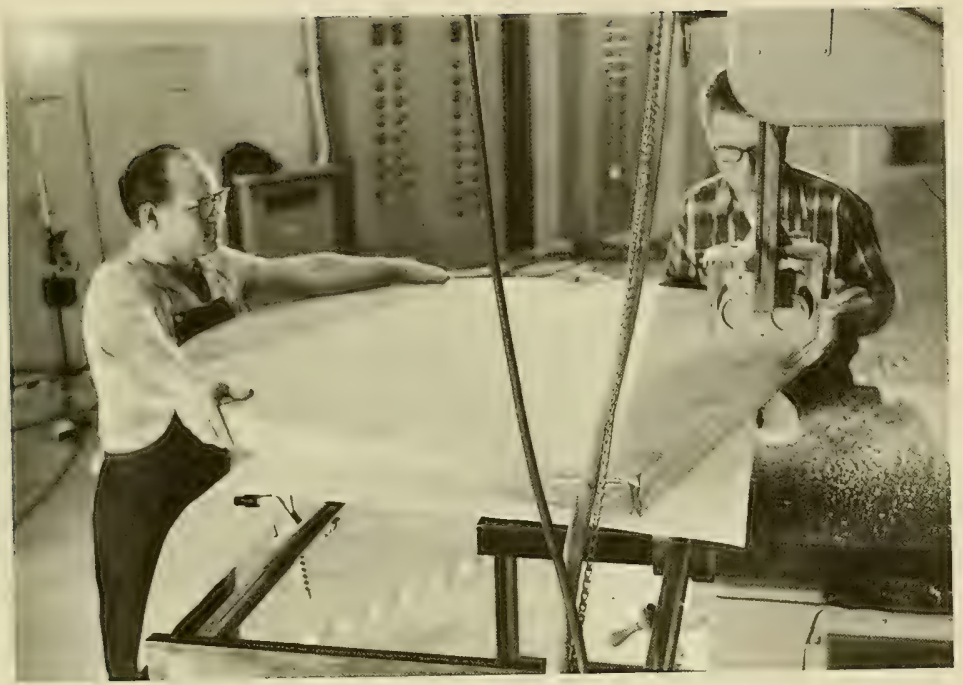

Figure 26. Sawing acrylic plastic plate stock into circular discs.

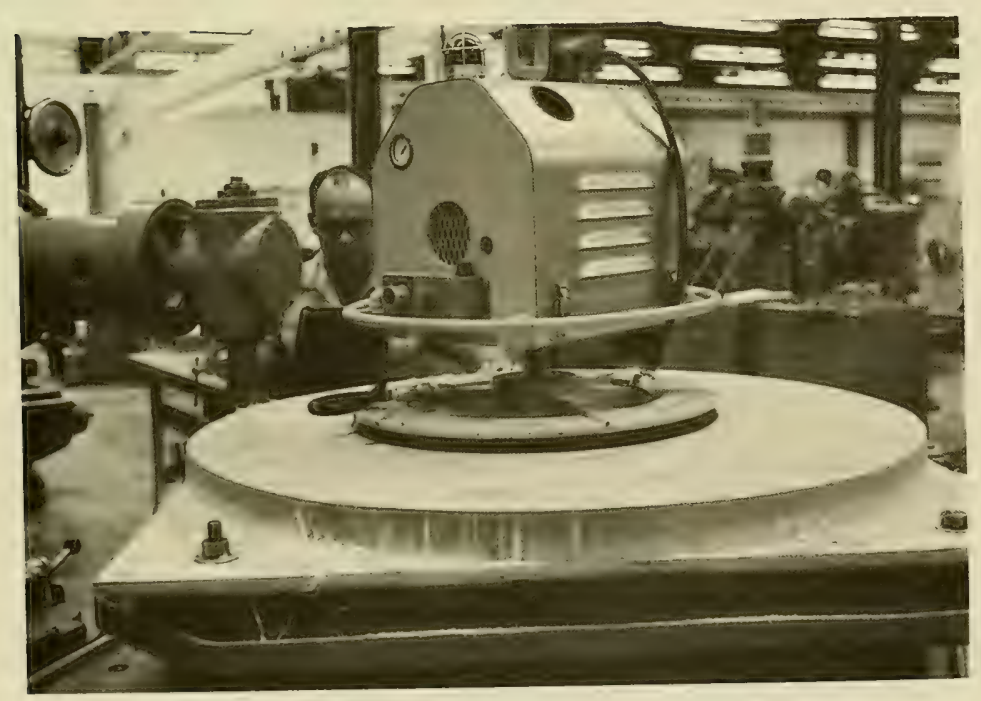

Figure 27. Turning edge of circular disc prior to placement in mold. 


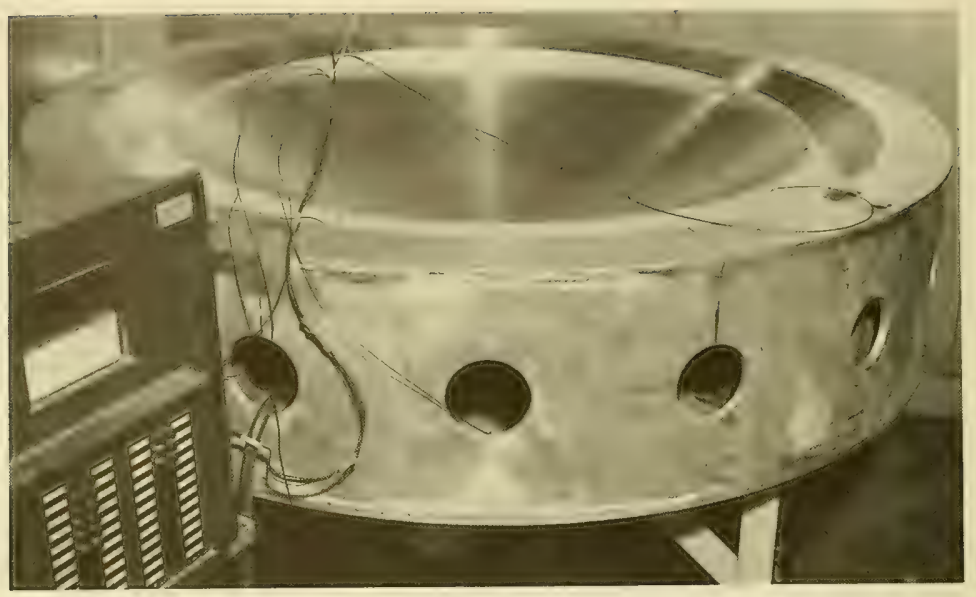

Figure 28. Circular disc after thermoforming at $300^{\circ} \mathrm{F}$ into spherical sector with a 33-inch radius of curvature; note thermocouples embedded in acrylic plastic for measurement of temperature.

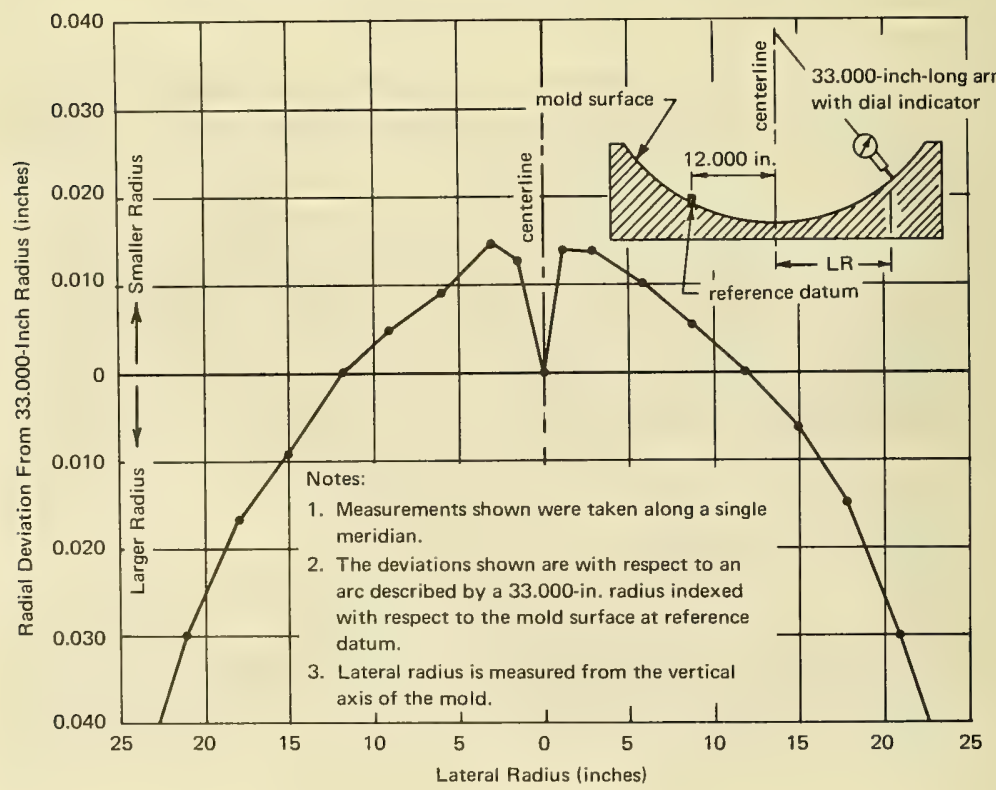

Figure 29. Actual sphericity of the mold for thermoforming sectors with 33-inch radius of curvature. 


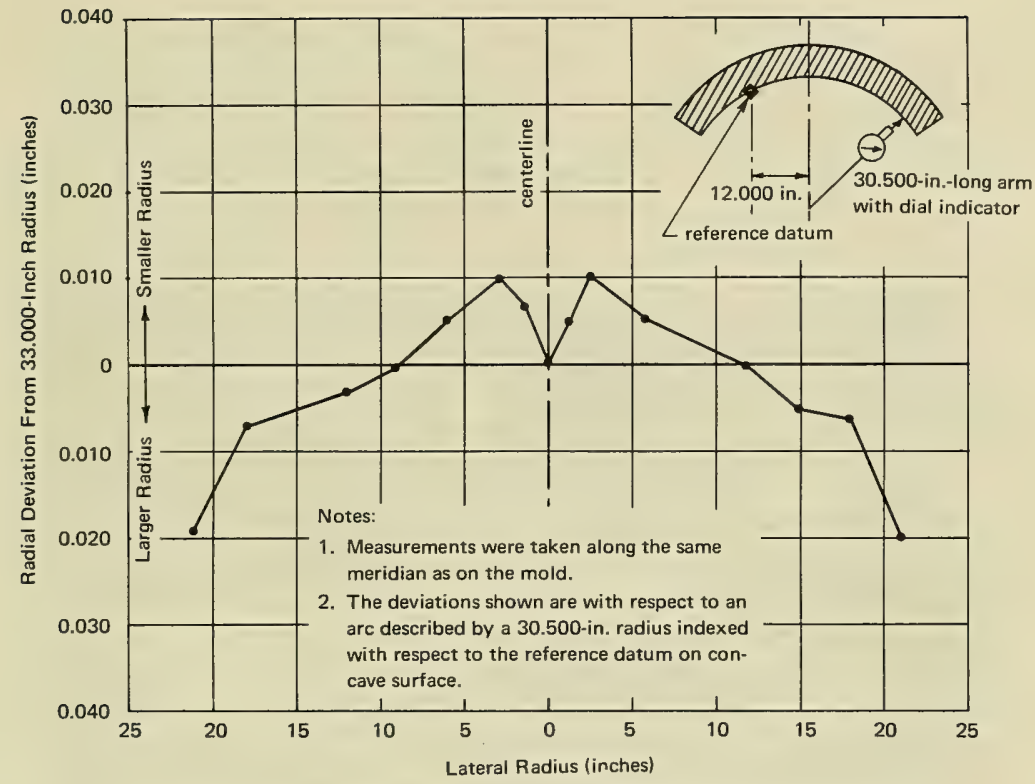

Figure 30a. Typical deviations from nominal 33-inch radius of curvature on thermoformed sector 18 for the 66-inch-diameter capsule.

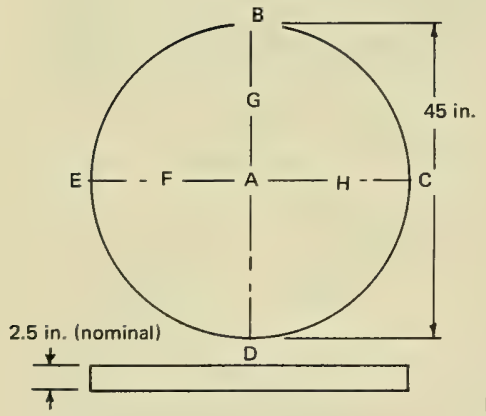

Location

A

B

C

D

E

F

G

H

I

\section{Thickness Before Forming (in.)}

2.390

2.467

2.502

2.477

2.404

2.430

2.432

2.425

2.430
Thickness After

Forming (in.)

2.362

2.556

2.592

2.593

2.477

2.410

2.425

2.404

2.420

Note: The increase in thickness is caused by lateral shrinking of previously "unshrunk" grade G Plexiglas plate after it was subjected to $300^{\circ} \mathrm{F}$ forming temperature. The thinning out of the formed sector at the center is due to stretching of acrylic as the previously flat plate is forced to conform to the spherical surface of the mold.

Figure $30 \mathrm{~b}$. Effect of thermoforming on thickness of sector 18 for the 66-inch-diameter capsule. 
In view of the fact that the deviations from sphericity of the formed sector were less than $1.0 \%$ of the specified radius, no real incentive existed to modify at that time the existing curvature of the mold by remachining it to an exact 33 -inch radius. Besides, it was predicted (and subsequently verified by actual measurements) that the deviations from specified sphericity during the thermoforming process are minor compared to the deviations resulting from machining of the pentagons. However, the generated data on deviations in sphericity proved conclusively that specifying the nominal radius of curvature of the mold 33.187 instead of 33.000 inches produced thermoformed sectors with radii of curvature larger rather than smaller than the specified 33.000 inches. Thus, it is recommended that before additional 66 -inch acrylic plastic spheres are fabricated, the curvature of the female mold be remachined accurately to the specified radius of curvature for the sphere.

Machining. The transformation of the spherical sectors into spherical pentagon modules was performed in two steps. The first step consisted of bandsawing the spherical sector into an oversized spherical pentagon (Figure 31). The second step, reducing the oversized spherical pentagon to the dimensions calculated for the spherical pentagon module, was performed on a vertical mill. For machining of model spherical pentagons, a manually operated mill was utilized (Figure 32), while for the machining of full-scale pentagons, a magnetictape-controlled mill was chosen (Figure 33). To facilitate the holding of the 33-inch-radius spherical sector in the bandsaw and in the vertical mill, a vacuum chuck (Figure 34) was designed and built by the shop personnel at the Pacific Missile Range. This chuck not only held the spherical sector in place but also served as a pneumatically operated indexing head for accurately locating the five straight beveled edges of the pentagon.

The machining of the large pentagons was performed with a 2-inchdiameter, helical-type milling cutter rotating at $3,800 \mathrm{rpm}$ and fed at $10 \mathrm{in} . / \mathrm{min}$ (Figure 33). A detergent and water cutting fluid was used to keep the cutter and the work piece cool so that serious stress concentrations would not be introduced into the spherical pentagon. The selected cutter, rotational speed, feed rate, and coolant resulted in a 63-rms finish for the beveled edges of the spherical pentagon.

Regardless of whether the pentagons were machined manually, as was the case with the model scale ones, or by preprogramed tape, as with the large pentagons, each pentagon had to be dimensionally checked before acceptance. The dimensional check consisted of measuring the linear distance between opposite tips of the pentagon with a specially built caliper having a 46-inch throat (Figure 35). Only if all the measurements between the opposite tips of the pentagon were within the specified dimensional range was the pentagon considered completed. To reduce the influence of temperature on the measurements, all readings were taken in the 65 to $75^{\circ} \mathrm{F}$ range. Although in the 
beginning of the machining operation grave doubts existed whether pentagons could be machined to the dimensional tolerances specified (Appendix A) for the prototype 66 -inch sphere, they soon were dispelled by these dimensional checks: the dimensional tolerances specified could be maintained. After the dimensions were checked, the large spherical pentagons were placed into contoured, padded plywood boxes (Figure 36); the model pentagons were stacked in shop baskets.

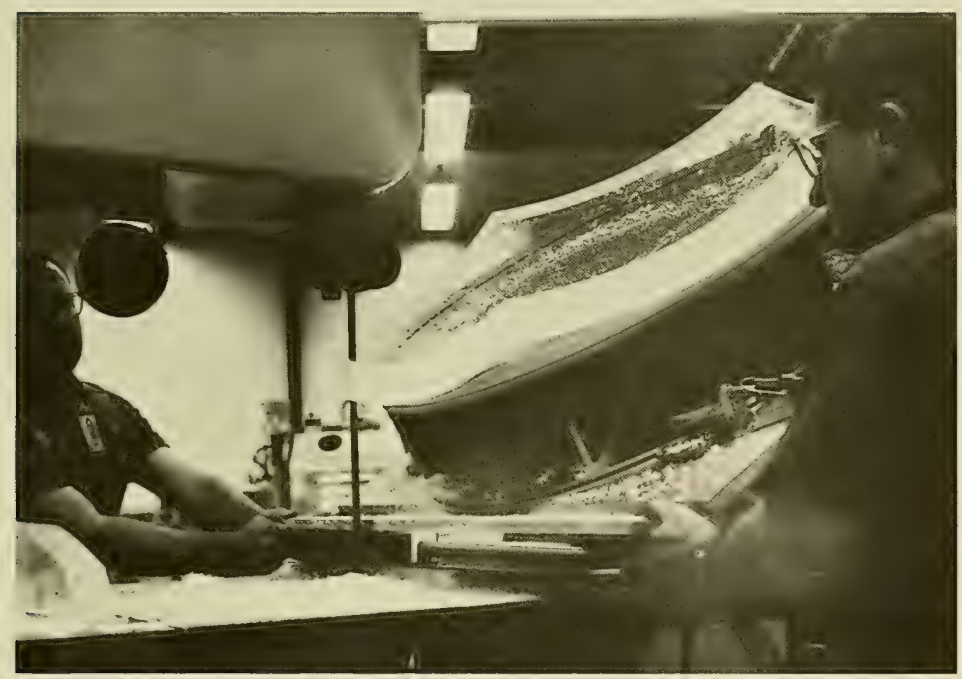

Figure 31. Sawing spherical sectors into rough spherical pentagon shape for the 66-inch-diameter capsule.

Annealing. No annealing of the model pentagons was performed after machining, as there were no contoured boxes or portable molds to support them during annealing. The annealing of the model pentagons was performed only after complete assembly and bonding of the model spheres, when the individual spherical pentagons could not readily change their curvature.

Large-scale spherical pentagons with a 33-inch spherical radius were annealed after completion of all the machining operations. This was performed to decrease the residual stresses introduced into the acrylic plastic by thermoforming, bandsawing, and milling. It was hoped that the decrease, if not complete elimination, of tensile stresses on the acrylic plastic surfaces would eliminate the tendency of acrylic plastic to craze on the machined surfaces. 


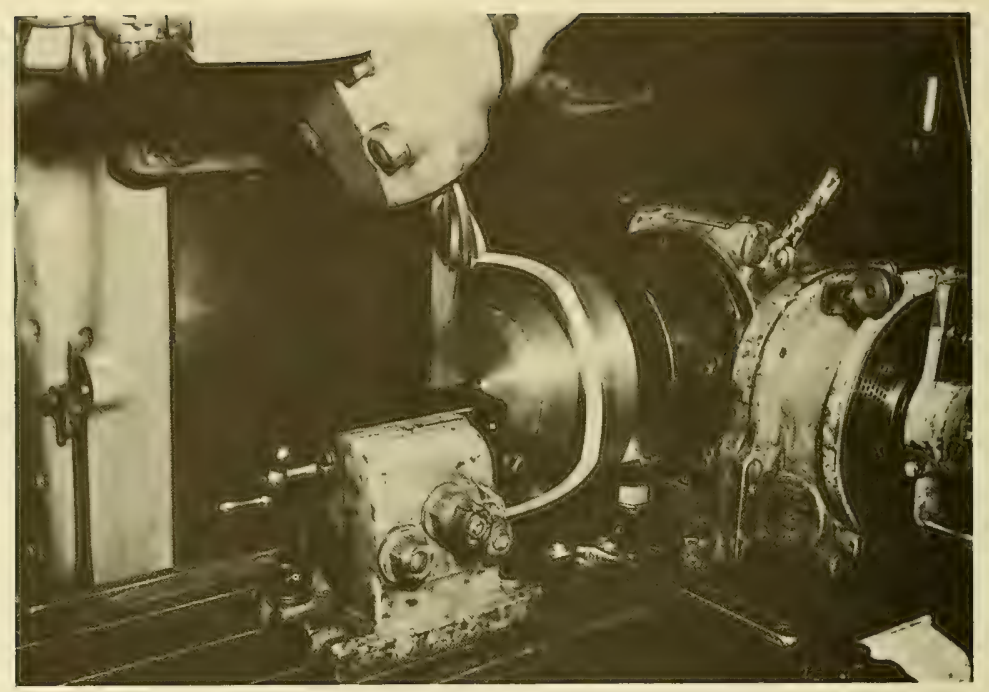

Figure 32. Milling the edges of spherical pentagon for the 15-inch-diameter capsule.

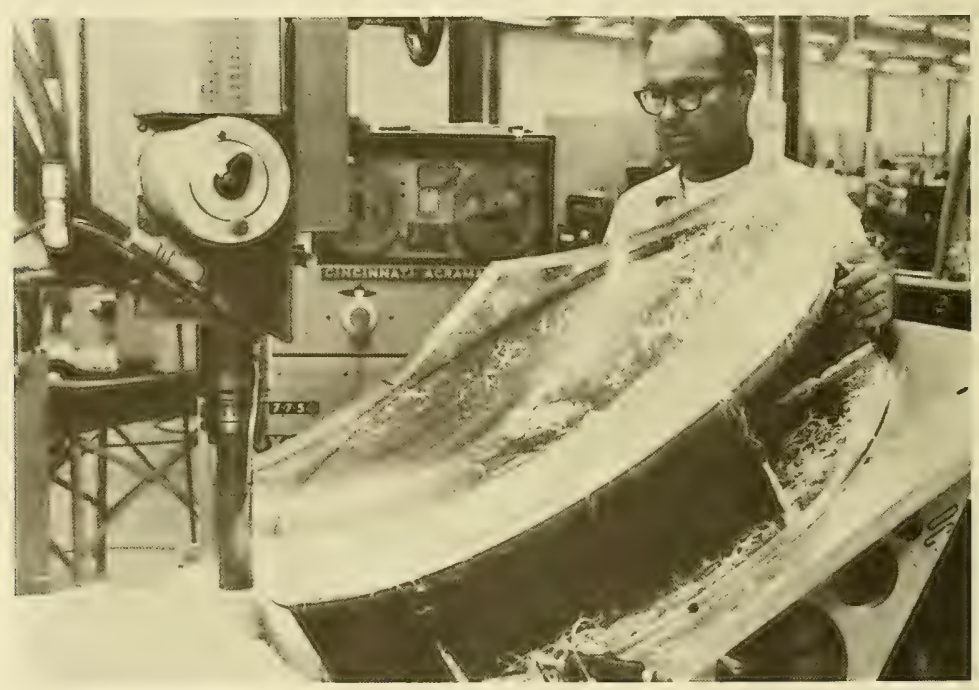

Figure 33. Milling the edges of spherical pentagon for the 66-inch-diameter capsule. 


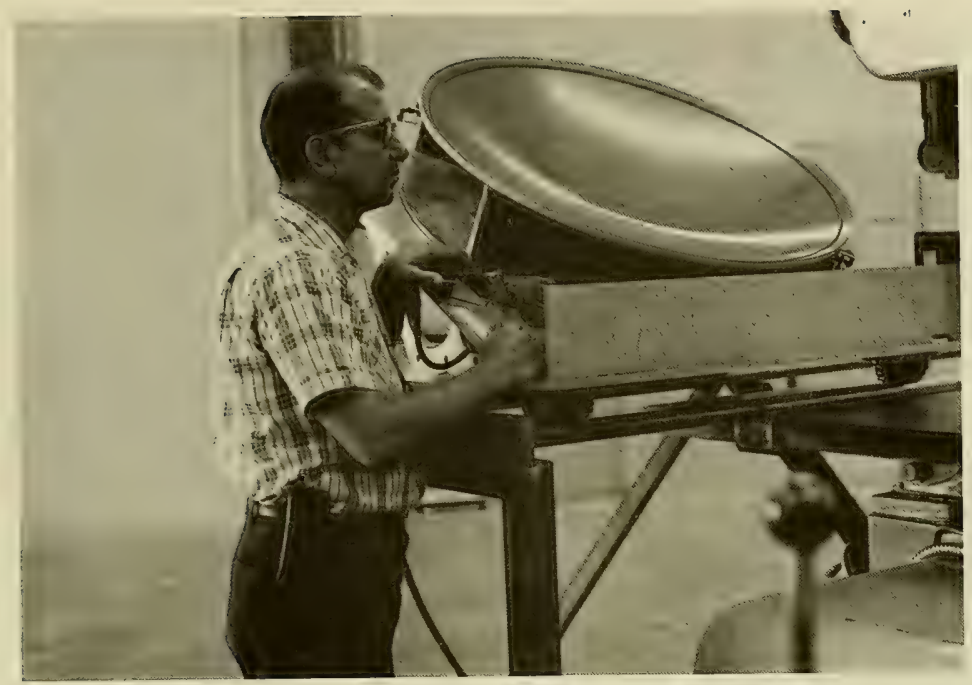

Figure 34. Indexing head with a vacuum operated chuck for the large spherical pentagons.

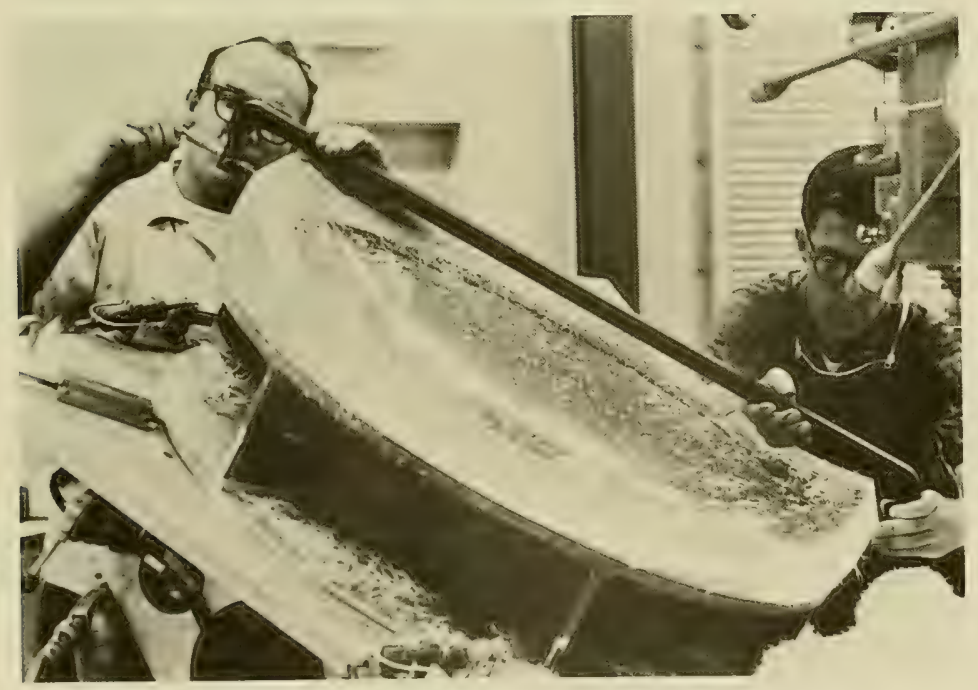

Figure 35. Dimensional check of large spherical pentagon prior to removing it from chuck. 


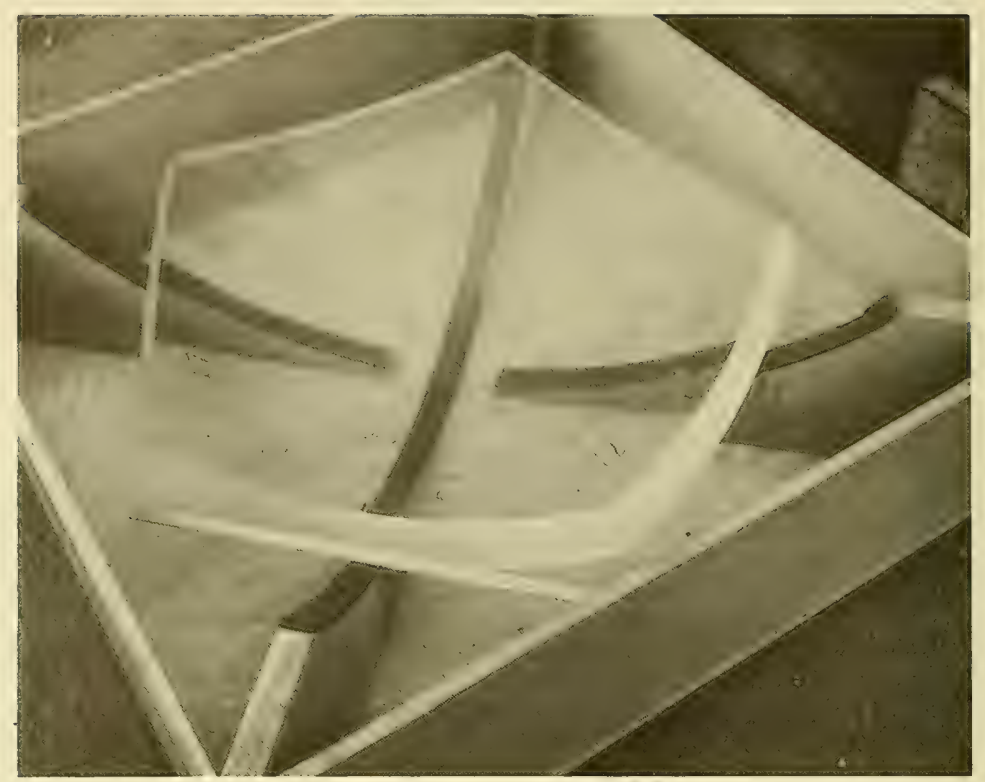

Figure 36. Storage of completed large pentagons between individual steps of the fabrication process.

Annealing of the large pentagons was performed in a walk-in oven. Each pentagon was placed there individually for 24 hours either in a wooden storage box, or in the mold, while the temperature was maintained at $160^{\circ} \mathrm{F}$. After the annealing period, the temperature in the oven was slowly reduced to $100^{\circ} \mathrm{F}$ at which time the pentagon was removed from the oven.

Quality Control. Since it was predicted prior to the machining and annealing operations that some realignment of the curvature would take place on the full-scale pentagons when residual stresses were relieved, a dimensional check was made on all pentagons after all major steps in their fabrication were completed (Appendix A). Thus, a measurement of the sphericity and thickness was made on all spherical pentagons after annealing, but prior to assembly and bonding into a sphere. The effects of bandsawing, milling, and annealing on the sphericity of the pentagon can be seen by comparing the sphericity of the first thermoformed spherical sector (Figure 30a) to the sphericity of the annealed pentagon made from that sector (Figure 37). 


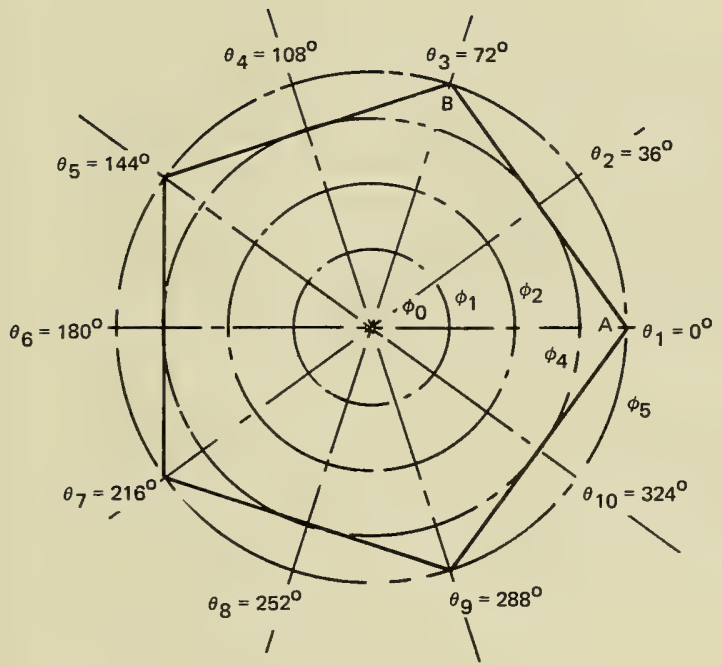

Typical Pentagon

$\begin{array}{ll}\frac{\phi \text { angle }}{\phi_{0}=0^{\circ} 0^{\prime}} & \phi_{4}=31^{\circ} 30^{\prime} \\ \phi_{1}=10^{\circ} 30^{\prime} & \phi_{5}=37^{\circ} 10^{\circ} \\ \phi_{2}=21^{\circ} 0^{\prime} & \end{array}$

Note:

1. Points $A$ and $B$ were marked to give orientation.

2. Angles $\phi_{1}$ through $\phi_{5}$ are angles through the center of the sphere.

3. Angles $\theta_{1}$ through $\theta_{10}$ are measured from the center of the pentagon.

4. Pentagons are for the prototype 66-inch NEMO capsule.

5. $\Delta R$ denotes change in internal surface curvature from specified 30.500 inches; + indicates shorter radius, while - indicates longer radius.

\begin{tabular}{|c|c|c|}
\hline \multirow{2}{*}{ Point } & \multicolumn{2}{|c|}{ Pentagon 18} \\
\hline & $\Delta R$ & Thickness \\
\hline$\theta_{1}-\phi_{0}$ & +0.007 & 2.385 \\
\hline$\theta_{1}-\phi_{1}$ & -0.018 & 2.405 \\
\hline$\theta_{1}-\phi_{2}$ & -0.045 & 2.435 \\
\hline$\theta_{1}-\phi_{4}$ & -0.089 & 2.485 \\
\hline$\theta_{1}-\phi_{5}$ & -0.127 & 2.510 \\
\hline$\theta_{2}-\phi_{1}$ & 0 & 2.405 \\
\hline$\theta_{2}-\phi_{2}$ & -0.015 & 2.435 \\
\hline$\theta_{2}-\phi_{4}$ & -0.065 & 2.465 \\
\hline$\theta_{3}-\phi_{1}$ & +0.015 & 2.400 \\
\hline$\theta_{3}-\phi_{2}$ & +0.013 & 2.405 \\
\hline$\theta_{3}-\phi_{4}$ & -0.038 & 2.435 \\
\hline$\theta_{3}-\phi_{5}$ & -0.083 & 2.470 \\
\hline$\theta_{4}-\phi_{1}$ & +0.023 & 2.390 \\
\hline$\theta_{4}-\phi_{2}$ & +0.017 & 2.400 \\
\hline$\theta_{4}-\phi_{4}$ & -0.024 & 2.420 \\
\hline$\theta_{5}-\phi_{1}$ & +0.018 & 2.395 \\
\hline$\theta_{5}-\phi_{2}$ & +0.017 & 2.405 \\
\hline$\theta_{5}-\phi_{4}$ & -0.039 & 2.450 \\
\hline$\theta_{5}-\phi_{5}$ & -0.100 & 2.475 \\
\hline$\theta_{6}-\phi_{1}$ & +0.004 & 2.395 \\
\hline$\theta_{6}-\phi_{2}$ & +0.020 & 2.400 \\
\hline$\theta_{6}-\phi_{4}$ & -0.073 & 2.460 \\
\hline$\theta_{7}-\phi_{1}$ & -0.010 & 2.400 \\
\hline$\theta_{7}-\phi_{2}$ & -0.014 & 2.430 \\
\hline$\theta_{7}-\phi_{4}$ & -0.134 & 2.570 \\
\hline$\theta_{7}-\phi_{5}$ & -0.187 & 2.550 \\
\hline$\theta_{8}-\phi_{1}$ & -0.023 & 2.415 \\
\hline$\theta_{8}-\phi_{2}$ & -0.070 & 2.485 \\
\hline$\theta_{8}-\phi_{4}$ & -0.168 & 2.555 \\
\hline$\theta_{9}-\phi_{1}$ & -0.028 & 2.428 \\
\hline$\theta_{9}-\phi_{2}$ & -0.074 & 2.485 \\
\hline${ }^{\theta} 9-\phi_{4}$ & -0.140 & 2.545 \\
\hline$\theta_{9}-\phi_{5}$ & -0.180 & 2.560 \\
\hline$\theta_{10}-\phi_{1}$ & -0.029 & 2.415 \\
\hline$\theta_{10}-\phi_{2}$ & -0.065 & 2.410 \\
\hline${ }^{\theta} 10^{-\phi_{4}}$ & -0.120 & 2.515 \\
\hline
\end{tabular}

Figure 37. Typical deviations from nominal 33-inch radius of curvature on a completed spherical pentagon for a 66-inch diameter capsule. 
Several conclusions can be made from that comparison. First, the deviations from specified sphericity are larger after machining operations than prior to them by a factor of 2 to 4 . Second, even with the considerable increases in spherical deviations, they are less than $1 \%$ of specified radius.

Similar measurements (Appendix C) were taken on model pentagons (Figure 38). The only difference here was that the measurements did not reflect any deviations from sphericity due to annealing process, as the model pentagons were not annealed until they were assembled and bonded into spheres.

Bonding. Initially the assembly of both the models and the 66-inch spheres was planned to be by the solvent-cement technique. The arguments for this bonding technique were that (1) the tensile strength of solvent-bonded joints can be made to equal that of the parent material by proper curing schedule, (2) all the operational parameters of this bonding technique are well known, and (3) the joint, if properly made, is completely colorless and transparent.

Although the arguments cited in support of this technique are valid, the results from bonding the first model capsule were quite disappointing. Either the joints were full of air cavities (Figure 39) and surfaces unwetted by the solvent because of insufficient clearance between the individual pentagon for penetration of solvent, or the joints were completely wetted by the solvent, but because of too large a separation between pentagons, the solvent upon drying left a very weak joint.

The reasons for this unsatisfactory performance of solvent-cement technique were many. The major ones were (1) all the pentagons had to be bonded simultaneously so that mismatches between individual pentagons could be distributed over the whole sphere, (2) edges of pentagons were not presoaked in solvent prior to assembly because of physical impossibility of presoaking the five edges of all pentagons simultaneously prior to assembly, (3) joint clearances and clamping forces varied from point to point depending on the dimensional deviation from nominal pentagon dimensions, and (4) it was nearly impossible to apply a calibrated clamping restraint on the whole sphere during bonding.

In order to improve the quality of the joints, an attempt was made to control the clearances in the joints so that complete penetration of the joints by solvent would take place. After an exploratory investigation into this problem, it was found that a 0.005 -inch clearance was required to create the capillary force that would draw the solvent into alt the joint spaces. Unfortunately, the joint with a 0.005 -inch clearance maintained by spacers had nearly zero strength as the evaporation of the solvent from the wide joint resulted in very inferior joint bond strength. The decrease of the joint clearance to 0.001 inch by placement of 0.001 -inch-thick spacers resulted in higher joint bond strength, but did not permit the solvent to wet all the joint surfaces. 


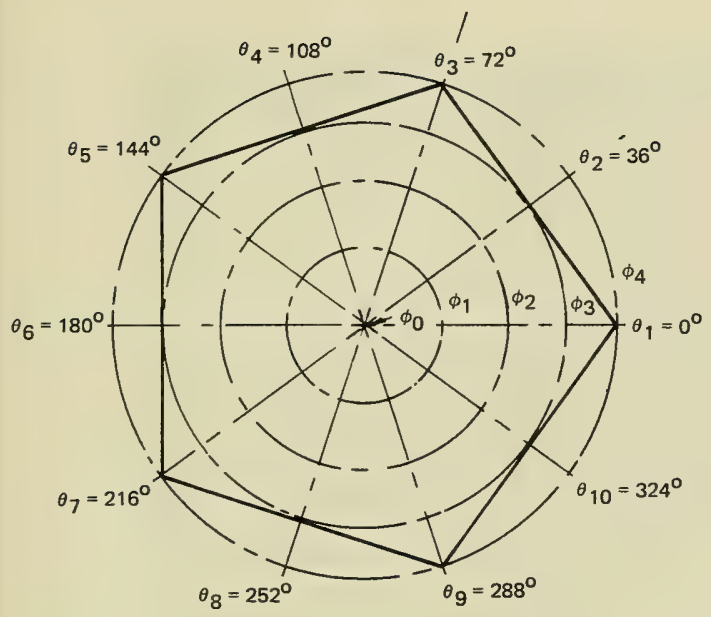

Note:

1. Mark on each pentagon the pentagon number at center and angle numbers at edge. These numbers should be maintained throughout fabrication of complete sphere.

2. Angles $\phi_{1}$ through $\phi_{4}$ are angles through center of sphere.

3. Angles $\theta_{1}$ through $\theta_{10}$ are measured from the center of pentagon.

4. $\Delta r$ denotes change in internal surface curvature from specified 7.000 inches; + indicates shorter radius, while - indicates longer radius.

$$
\begin{aligned}
& \phi \text { angle } \\
& \phi_{0}=0^{\circ} 0^{\prime} \\
& \phi_{1}=10^{\circ} 30^{\circ} \\
& \phi_{2}=21^{\circ} 0^{\circ} \\
& \phi_{3}=31^{\circ} 30^{\circ} \\
& \phi_{4}=37^{\circ} 10^{\circ}
\end{aligned}
$$

\begin{tabular}{|c|c|c|}
\hline Point & $\Delta r$ & Thicknets \\
\hline$\theta_{1}-\phi_{0}$ & +0.003 & 0.485 \\
\hline$\theta_{1}-\phi_{1}$ & +0.0015 & 0.475 \\
\hline$\theta_{1}-\phi_{2}$ & 0.000 & 0.475 \\
\hline$\theta_{1}-\phi_{3}$ & -0.006 & 0.475 \\
\hline$\theta_{1}-\phi_{4}$ & -0.018 & 0.485 \\
\hline$\theta_{2}-\phi_{1}$ & +0.0015 & 0.475 \\
\hline$\theta_{2}-\phi_{2}$ & 0.000 & 0.475 \\
\hline$\theta_{2}-\phi_{3}$ & -0.011 & 0.475 \\
\hline$\theta_{3}-\phi_{1}$ & +0.0015 & 0.470 \\
\hline$\theta_{3}-\phi_{2}$ & 0.000 & 0.472 \\
\hline$\theta_{3}-\phi_{3}$ & -0.004 & 0.475 \\
\hline$\theta_{3}-\phi_{4}$ & -0.018 & 0.485 \\
\hline$\theta_{4}-\phi_{1}$ & +0.002 & 0.472 \\
\hline$\theta_{4}-\phi_{2}$ & 0.000 & 0.472 \\
\hline$\theta_{4}-\phi_{3}$ & -0.009 & 0.470 \\
\hline$\theta_{5}-\phi_{1}$ & +0.0015 & 0.475 \\
\hline$\theta_{5}-\phi_{2}$ & 0.000 & 0.475 \\
\hline$\theta_{5}-\phi_{3}$ & -0.004 & 0.485 \\
\hline$\theta_{5}-\phi_{4}$ & -0.010 & 0.485 \\
\hline$\theta_{5}-\phi_{1}$ & +0.003 & 0.475 \\
\hline$\theta_{6}-\phi_{2}$ & 0.000 & 0.485 \\
\hline$\theta_{6}-\phi_{3}$ & -0.009 & 0.485 \\
\hline$\theta_{7}-\phi_{1}$ & +0.001 & 0.472 \\
\hline$\theta_{7}-\phi_{2}$ & 0.000 & 0.475 \\
\hline$\theta_{7}-\phi_{3}$ & -0.005 & 0.485 \\
\hline$\theta_{7}-\phi_{4}$ & -0.009 & 0.485 \\
\hline$\theta_{8}-\phi_{1}$ & +0.003 & 0.475 \\
\hline$\theta_{8}-\phi_{2}$ & 0.000 & 0.482 \\
\hline$\theta_{8}-\phi_{3}$ & -0.007 & 0.485 \\
\hline$\theta_{9}-\phi_{1}$ & +0.0015 & 0.475 \\
\hline$\theta_{9}-\phi_{2}$ & 0.000 & 0.480 \\
\hline$\theta_{9}-\phi_{3}$ & -0.004 & 0.487 \\
\hline$\theta_{9}-\phi_{4}$ & -0.009 & 0.492 \\
\hline$\theta_{10}-\phi_{1}$ & +0.0025 & 0.475 \\
\hline$\theta_{10}-\phi_{2}$ & 0.000 & 0.475 \\
\hline$\theta_{10}-\phi_{3}$ & -0.009 & 0.480 \\
\hline
\end{tabular}

Figure 38. Typical deviations from nominal 7.5-inch radius of curvature on a completed spherical pentagon for 15 -inch-diameter capsule model. 
A procedure was finally developed for solvent bonding model capsules that produced high-strength bonds over $100 \%$ of the joint area (Figure 40 ): (1) assemble the model capsule with 0.005 -inch spacers between pentagons, (2) place a clamping restraint on it in the form of rubber bands cut from auto inner tubes, (3) introduce solvent into the joint by means of a syringe (results in complete wetting of joint surfaces by capillary action), and (4) rapidly remove the spacers before the joint surfaces are bonded, thus permitting the clamping forces to bring the joint surfaces into intimate contact. This procedure was used to assemble and bond model NEMO capsules 1 through 11.

The annealing of the solvent-bonded model capsules consisted of placing them in an oven heated to $175^{\circ} \mathrm{F}$ and leaving them there for 24 hours at that temperature. The annealing process, however, did not eliminate completely the residual stresses in the joints introduced into the capsule by the bonding process (Figure 41). Since it was felt that the remaining residual stresses in the model capsule (approximately 1,300-psi shear stress in the joint area) would not significantly decrease the implosion pressure of the capsule, no further effort was made to eliminate the residual stresses after the single annealing operation described.

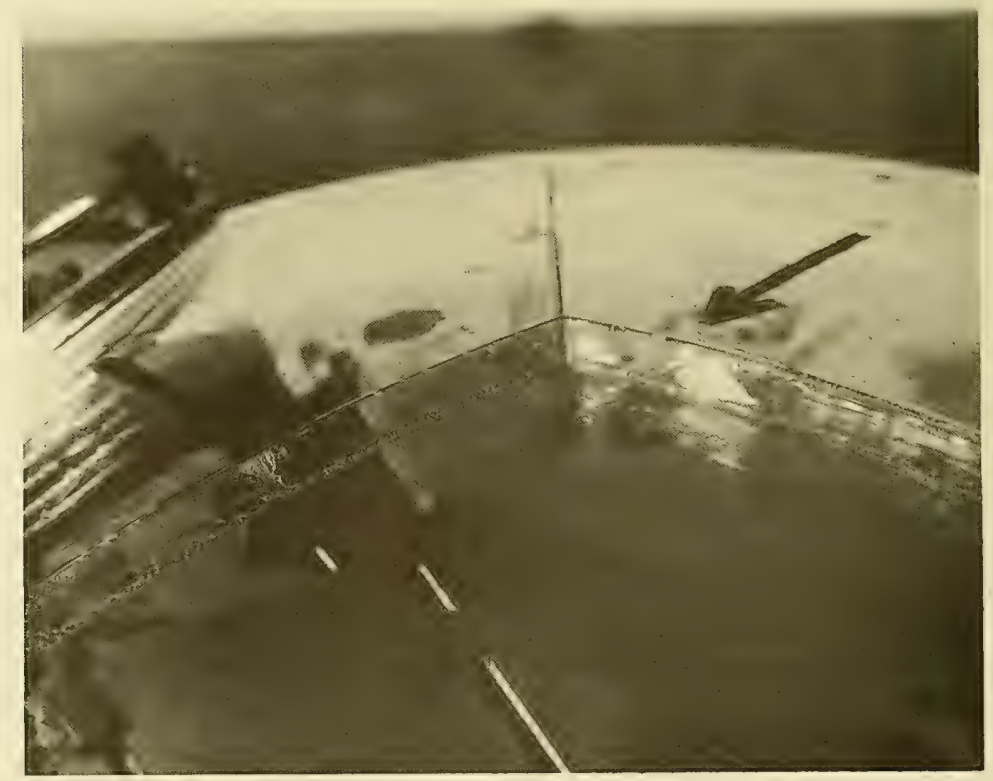

Figure 39. Typical air bubbles in an improperly solvent-bonded joint on the 15-inch-diameter capsule model. 


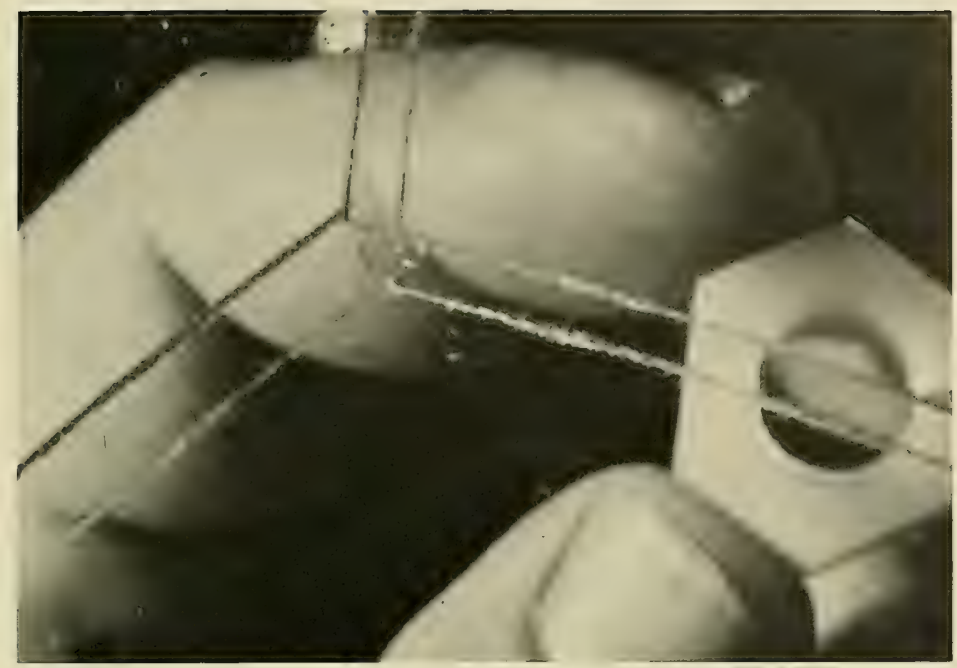

Figure 40. Typical sample of a properly solvent-bonded joint on a 15-inch-diameter capsule model.

Although the procedure developed for solvent bonding model capsules produced satisfactory joints, grave doubts existed whether this technique would produce satisfactory results for the large-scale capsule. It appeared that in order to solvent bond the large pentagons, the dimensional control of individual pentagons would have to be so strict as to make the fabrication cost unacceptable. Also, the cost of a clamping system for placing the solvent-bonded joints in a large sphere under uniform compression would, in all probability, be higher than the cost of fabrication up to the bonding stage. In view of these problems, it was decided to find a different joint bonding technique that, although being as costly as the solvent bonding technique for the models, would be distinctly less for the large-scale capsule.

The alternate bonding technique investigated was the cast-in-place adhesive. The prime characteristics of this bonding technique are: (1) the pieces to be bonded are maintained in a fixed relationship to each other by spacers that do not permit the pieces to contact each other, (2) the space between the acrylic plastic members is made fluid tight by placement of adhesive tapes over both sides of the space, (3) the adhesive is poured into the taped-over space by means of a funnel or squeeze bottle, and (4) filling the joint space is done in more than one pour, with the mechanical spacer being removed from the joint after the first increment of adhesive-filled joint has set, but before the succeeding increment of adhesive covers it (Figure 42). 


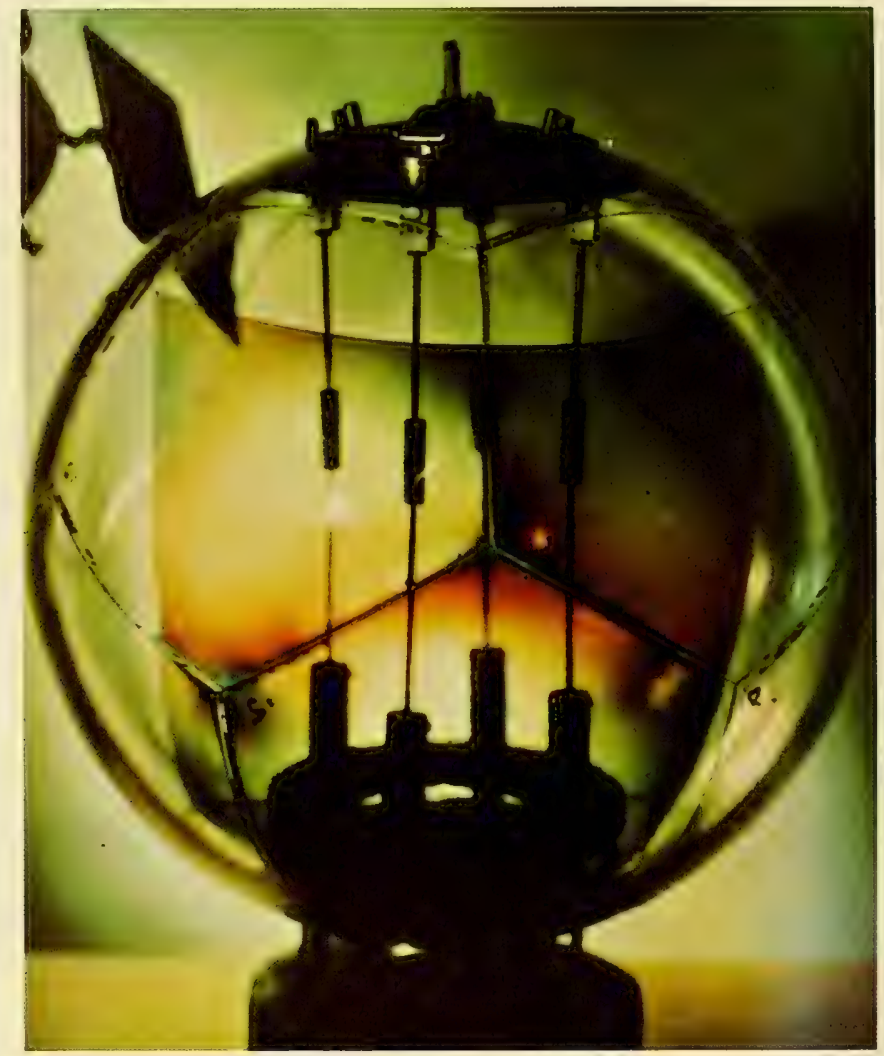

Figure 41. Residual stresses in a solvent-bonded 15-inch-diameter capsule not removed by annealing operation; maximum shear stress approximately 1,300 psi.

The advantages of this bonding technique are: (1) the dimensional tolerances of spherical pentagons being bonded need not be tight because reasonable dimensional deviations are taken care of by the 0.100 -to-0.150inch-wide joint space, (2) there is no requirement for clamping forces on the members being bonded, (3) irregularly shaped surfaces can be joined, providing the joint space can be sealed on both sides with adhesive tape, (4) there is no requirement for fine finish on the acrylic plastic surfaces forming the two sides of the joint, and (5) the bonding of a large structure can proceed by small increments with each joint increment becoming set before the next pouring is performed. 


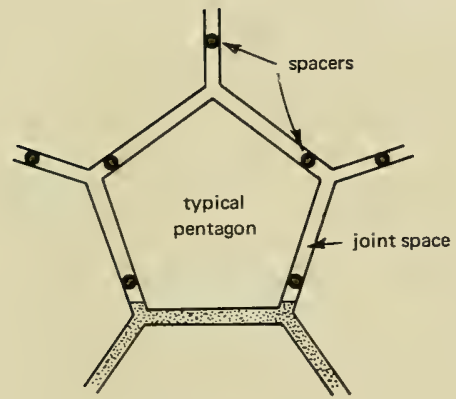

Adhesive Pour No. 1

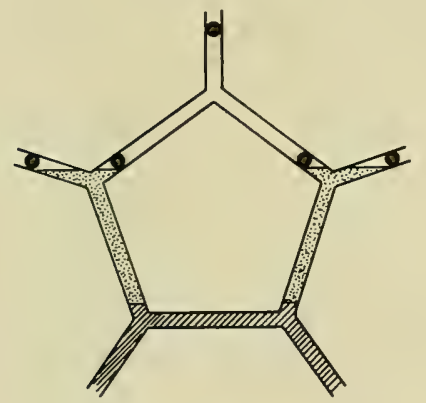

Adhesive Pour No. 2

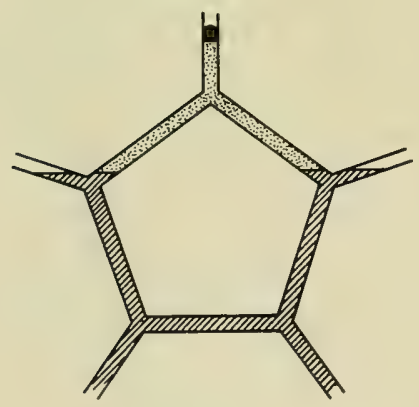

Adhesive Pour No. 3

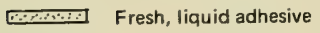

सIIسW Old, solid adhesive

Figure 42. Procedure for casting of self-polymerizing joints in acrylic plastic capsules.
In view of the many advantages that such a bonding technique possesses for fabrication of large-scale acrylic plastic capsules, it was decided to evaluate this technique first on small-scale capsules and on joint-test specimens simulating the joint dimensions of the 66inch capsule. Four cast-in-place adhesives were investigated: epoxy, polyester, PS-18 acrylic cement, and PS-30 acrylic cement. The epoxy and polyester adhesives were almost immediately discarded as the tensile strength of a joint between acrylic plastic members filled with these adhesives was less than 1,500 psi for epoxy and 1,000 psi for polyester. Although the compressive strength of these two adhesives was indistinguishable from that of the acrylic plastic parent material, the low tensile strength just made these adhesives noncompetitive with the PS-18 and PS-30 acrylic cements. When 2.5-inch-wide acrylic plastic blocks were bonded with acrylic cement in 0.125-inchwide joints, it was found that the tensile strength for PS-18 was consistently higher than 5,000 psi, while for PS-30 it was higher than 4,000 psi (Table 3). Compressive strength of these joints was found to be of approximately the same magnitude as that of the parent acrylic plastic material, but their deformation under load was somewhat higher. 


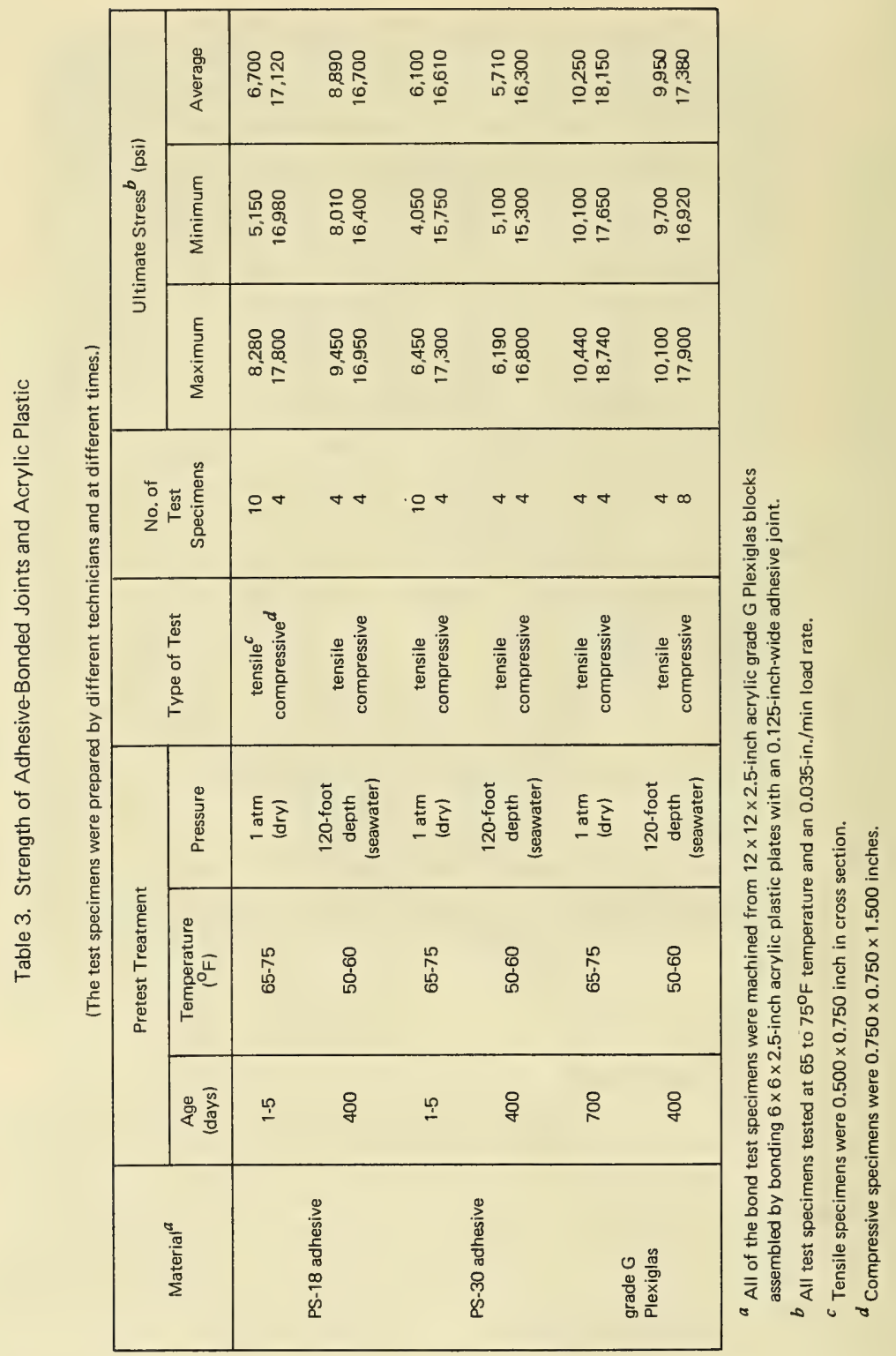


Since it was known that both PS-18 and PS-30 adhesive joints deteriorate in strength with age when exposed to atmospheric environment, it was questioned whether immersion in seawater accelerates this aging process further. To answer this question, a bonded acrylic plastic block was placed in the Pacific off Point Mugu at a depth of 120 feet. After 13 months of continuous submersion, the acrylic plastic block was retrieved, and material test specimens were machined from it. Because the block was rather small, and only a limited number of test specimens could be machined from it, only tensile and compressive test specimens were cut from it. These test specimens were tested at $70^{\circ} \mathrm{F}$ to establish the ultimate tensile and compressive strength of the PS-18 and PS-30 cast joint after 1 year's exposure to seawater.

When the test specimens were tested to destruction, it was found (Table 3 ) that the average tensile strength of the 0.125 -inch-wide, adhesivefilled joints was 8,890 psi for PS-18 and 5,710 psi for PS-30 joints. Since no control block was bonded at the time when the ocean test specimen was fabricated and placed in the ocean, no accurate comparison can be made with test specimens exposed for 1 year to atmospheric environment. A general comparison, however, can be made with tensile and compressive strengths of PS-18 and PS-30 adhesive-bonded joints tested several days after bonding. The comparison between strengths of the fresh joints and those 1-year old but kept submerged in the ocean showed that no statistically significant decrease in tensile or compressive strength occurred for PS-18 or PS-30 adhesive-bonded joints. As a matter of fact, it appears that the strength of a PS-18 adhesive-bonded joint increased somewhat because of long-term immersion in the ocean (Table 3).

Tests were conducted also on grade G Plexiglas material after immersion in the ocean for 13 months to verify tests conducted by others ${ }^{19,}, 20$ indicating that there is no statistically significant degradation of material properties in acrylic plastic after long-term immersion in the ocean even at depths in excess of several thousand feet. Taking into consideration the experimental findings that neither the acrylic plastic nor the PS-18 adhesive joints deteriorated in strength during a 1-year immersion in seawater, it was decided to proceed with the construction of the full-scale acrylic plastic capsule utilizing PS-18 adhesive.

Although no particular problems were encountered during bonding of PS-18 joint test specimens, it remained to be seen how well this bonding technique would work in practice while bonding the spherical pentagons in a capsule. For this reason it was decided to discontinue bonding model capsules with the solvent-cement technique and to bond all the subsequent model capsules with PS-18 cement. 
This was accomplished in the following manner: First, the spherical pentagons were made smaller (distance across tips decreased from 8.660 inches to 8.553 inches and true chord length from 5.352 inches to 5.286 inches) so that 0.125 -inch-wide joints could be incorporated between the individual pentagons in the assembled hull. Second, an assembly jig was utilized that, although initially developed for providing precise alignment and clamping of all pentagons during solvent-bonding of joints (Figure 43), could be also used for accurate alignment and support of six pentagons during bonding operations employing cast-in-place adhesive. Third, after removal of all spacers between pentagons, the joint spaces were taped over on the interior and exterior surfaces of the hull with Rohm \& Haas 670 cellophane fiber tape to contain the adhesive during casting. Fourth, the adhesive was mixed in the proportions recommended by the supplier, degassed under vacuum for approximately 15 minutes, and poured into the joint spaces by means of a gravity-fed funnel. The recommended procedure for mixing of PS-18 adhesive with a 60-minute pot life at room temperature was to dissolve one 2.4-gram capsule of catalyst (50-50 mix of granular benzoyl peroxide and camphor) in 113 grams of resin (methyl methacrylate monomer and methyl methacrylate polymer resin) followed by addition of $3 \mathrm{cc}$ of promoter to the two already mixed components. Since the spacers were removed prior to taping over the interior surface of the joints between the six pentagons, the adhesive could be placed into all the joints of a hemisphere in one pour. Thus the adhesive was mixed in one batch and placed into the joint spaces in a single continuous pour. Fifth, upon setting of the adhesive which occurred in approximately an hour, the tapes were stripped from the joints and the six pentagons bonded into a hemisphere were removed from the jig. A similar procedure was repeated with the other six pentagons. Upon completion of the second hemisphere, both hemispheres were placed upon each other separated by spacers which, prior to placement of the tape on the exterior of the joint, were removed. The joint between the hemisphere was subsequently filled with adhesive. Sixth, the model was placed into an oven for annealing at $175^{\circ} \mathrm{F}$ for 24 hours. The residual stresses remaining in the capsule after annealing were of approximately the same magnitude and uniformity (Figure 44) as those resulting from solvent-bonding of model capsules (approximately 1,300-psi shear stress in the joints).

The successful casting of joints in the model capsules and subsequent testing that showed no significant difference in structural response to hydrostatic pressure from models bonded with solvent cement proved conclusively that casting of PS-18 adhesive into 0.125-inch-wide joints (Figure 45) was acceptable from both fabrication and operational viewpoints and thus should be applied to bonding of large-scale acrylic plastic capsules. 


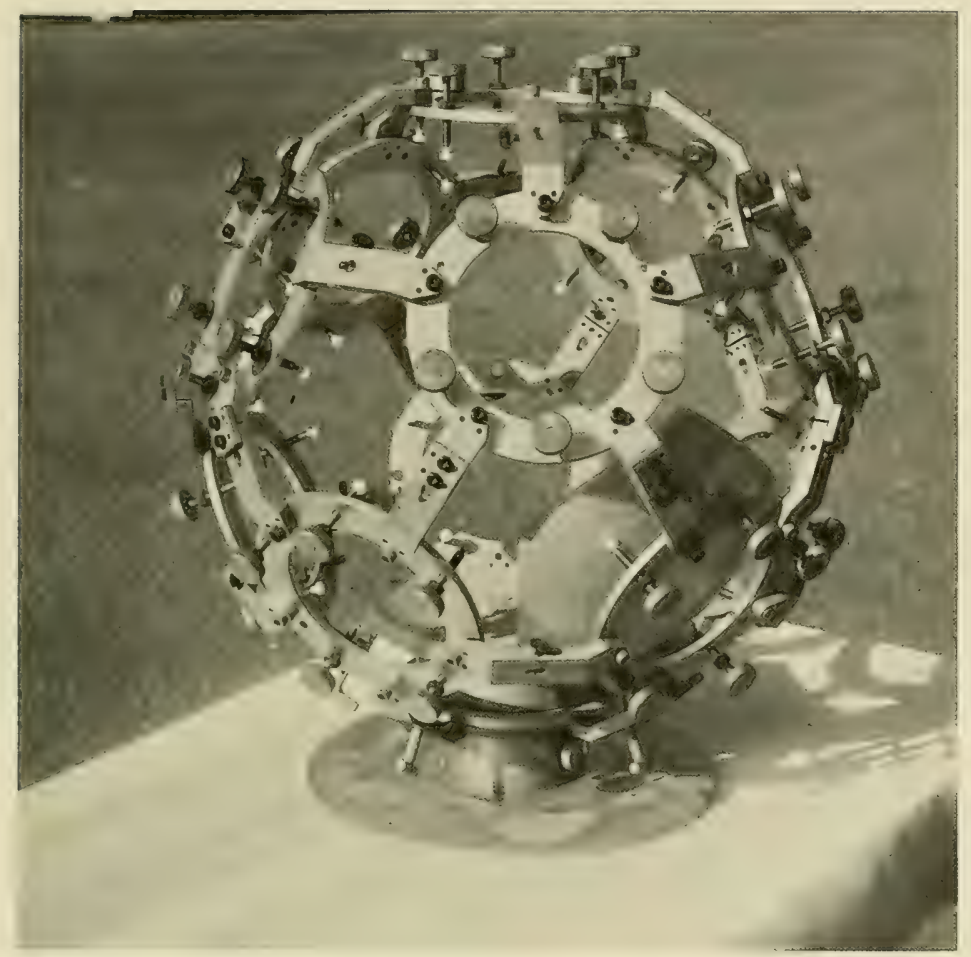

Figure 43. Jig for assembly of individual spherical pentagons into a 15-inch-diameter capsule.

Large-scale capsules were also fabricated from undersize spherical pentagons, that when assembled allowed an 0.125-inch joint space to exist between individual pentagons. Since the fabrication of an assembly jig for the large-scale capsule according to the same design that was used for the model scale capsule would have been expensive, a simpler jig was designed and built. The basic difference between the large-scale capsule jig and the model jig was that the large assembly jig permitted access for the shop personnel to the interior of the hemisphere to make the taping of joints on the interior easier.

The large-scale capsule was assembled and bonded like the models in three phases - the first two phases resulted in the assembly and bonding of pentagons into two hemispheres, while the third one culminated in the assembly of two hemispheres into a single sphere (Figures 46 through 50). 


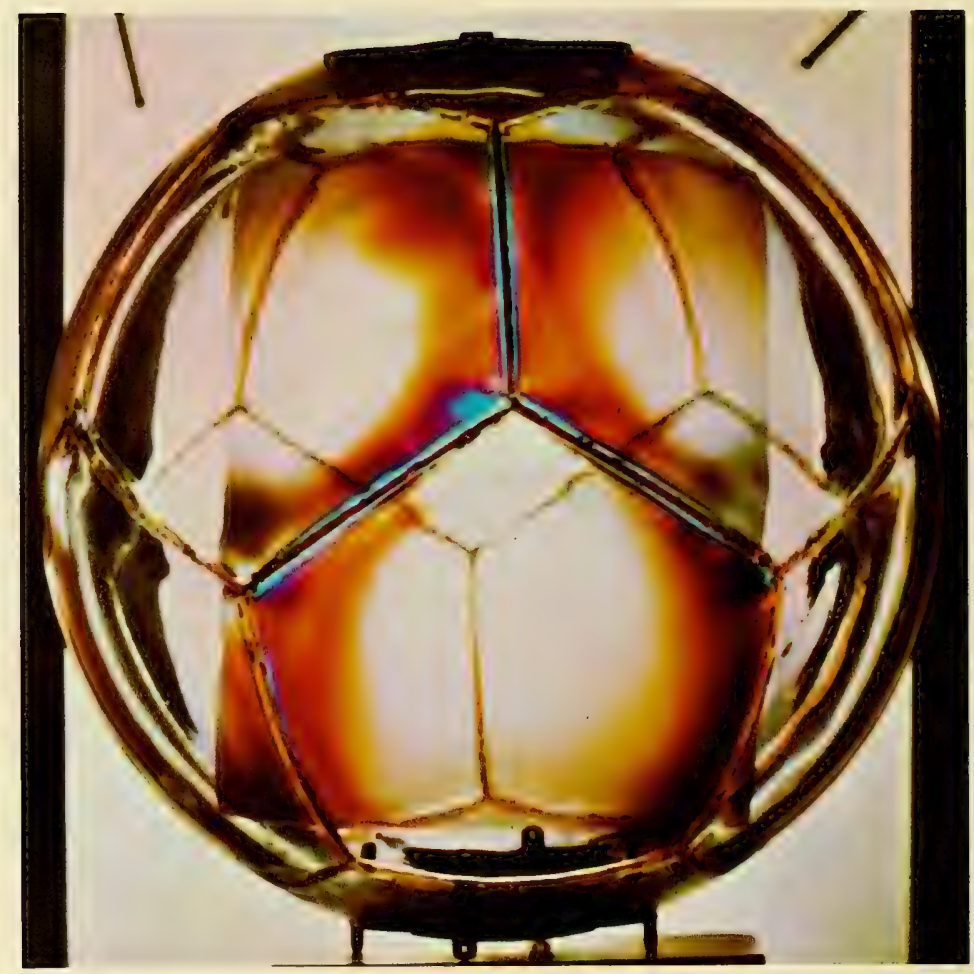

Figure 44. Residual stresses not removed by annealing in a 15-inch-diameter capsule with cast-in-place joints.

This method of assembling and bonding the capsule possessed several disadvantages not present in the method of assembly used on solvent-bonded models, which permitted bonding of all 12 pentagons in one operation. These disadvantages were (1) difficulty in checking the sphericity of the assembled hemisphere prior to bonding, (2) distribution of dimensional mismatches between individual pentagons over the surface of a hemisphere only, and (3) inability to accomplish a perfect match between the irregular joint surfaces of the two mating hemispheres. But it was hoped that with proper attention to the sphericity and diameter of each individual hemisphere, a capsule could be assembled with small deviations from nominal diameter and sphericity. 
Figure 45.

Typical sample of a properly cast-in-place joint in a 15 -inchdiameter capsule.
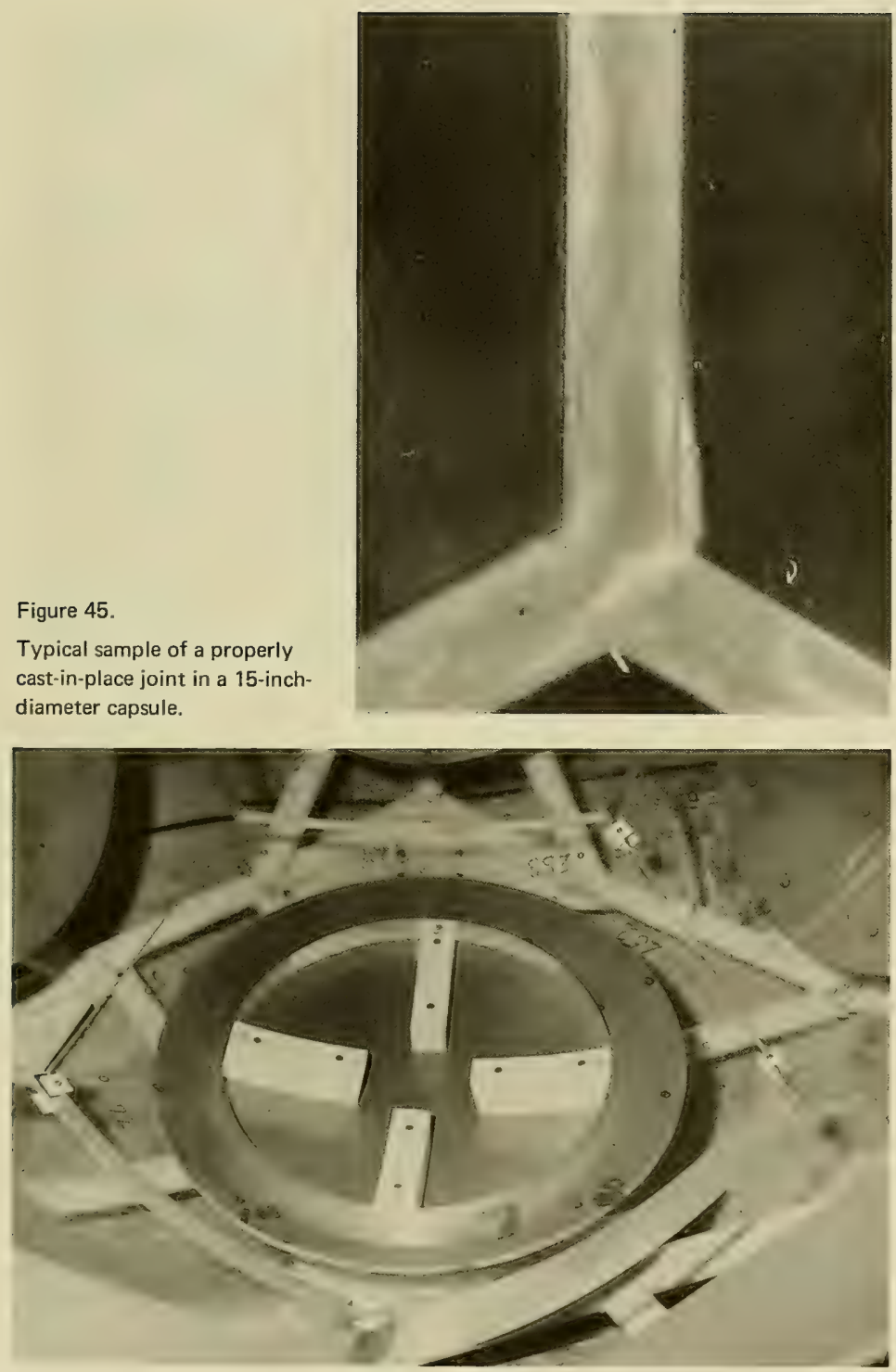

Figure 46. Jig with contoured spacers for centering the hemisphere for bonding. 


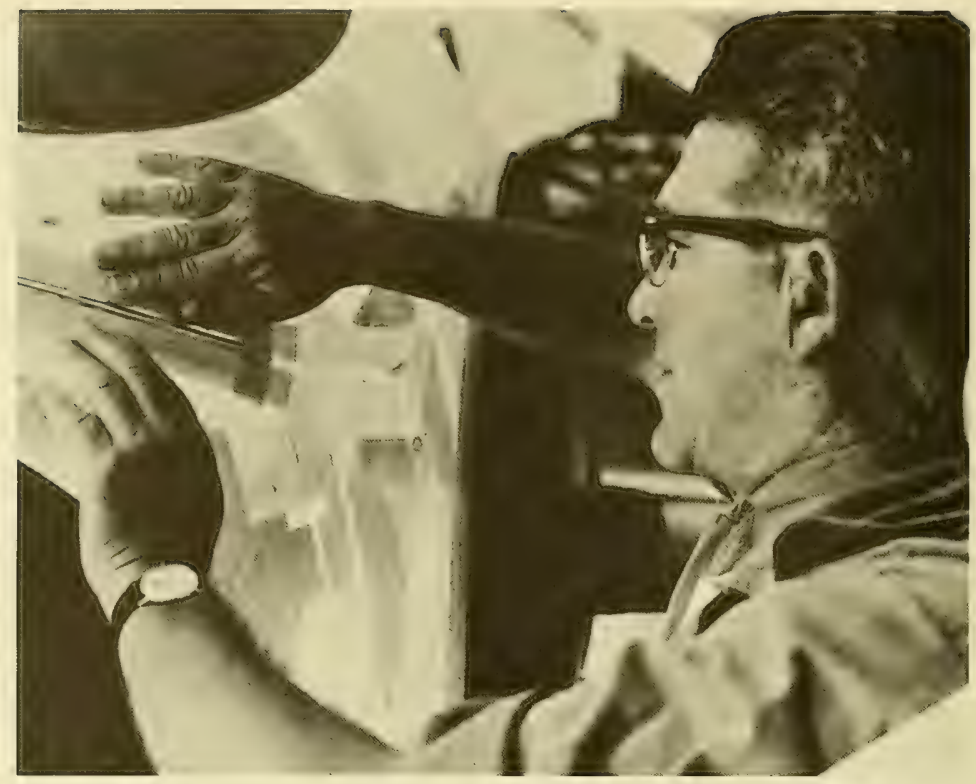

Figure 47. Sealing of joint space between spherical pentagons prior to bonding.

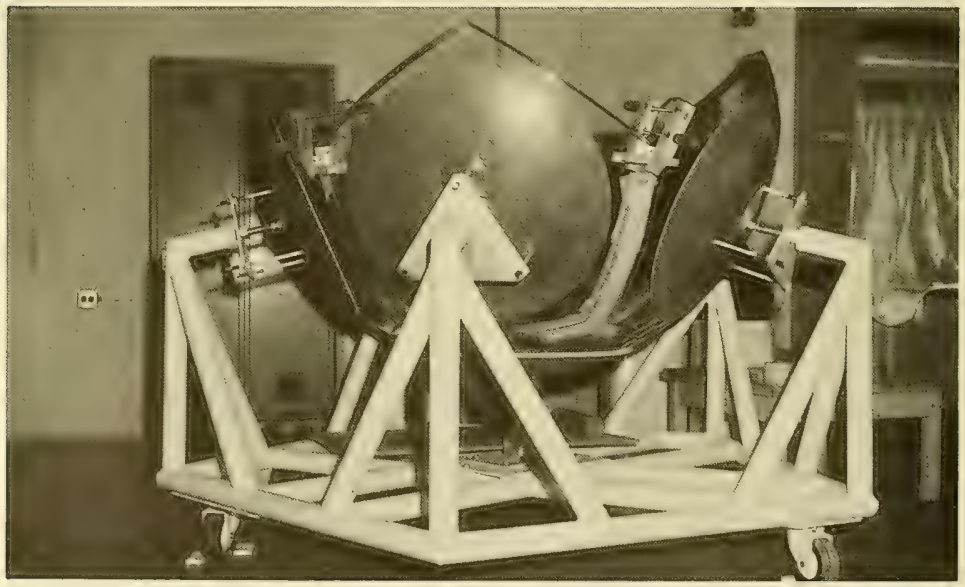

Figure 48. Acrylic plastic hemisphere assembled from six spherical pentagons prior to bonding operation. 
Although the preparation for bonding was almost identical to the one used for scale models, considerably more difficulties were encountered in the bonding of the large-scale capsule. These difficulties can be placed into two categories: (1) mixing and pouring of large quantities of adhesive and (2) elimination of cavities and separation spaces in cast joints. The problem in the first category centered around mixing large quantities of adhesive components without inclusion of air bubbles and at the right temperature so that the mix would not set during pouring into the joints while at the same time retaining the ability to set in the joint within a short span of time. If the chemical reaction in the mix progressed too rapidly (because of the large mass of adhesive involved) during pouring, it not only generated an excess of bubbles but the adhesive would set in some portions of the joint prior to filling it completely. When this occurred, access holes had to be drilled through already-set adhesive to permit trepanning the voids in joint spaces so that they could be filled with adhesive by means of a syringe. On the other hand, when the mix reacted so slowly that several hours were required before it set, the components of the mix attacked the acrylic plastic surfaces of the joint causing them to craze. Since the exact temperature was not known to which the components of the mix had to be precooled to prevent premature setting during pouring while still permitting rapid setting in joint, the trial and error approach was utilized. The problem of arriving at a satisfactory mix temperature was further complicated by the variation in ambient temperature during the several day-long periods required for casting the joints. As a result, the placement of PS-18 into the joint spaces was a continuous trial and error operation. Some of the joint sections were filled with the adhesive without any trouble, while others had voids or separation spaces that had to be laboriously drilled out and refilled because of the too-rapid or too-slow setting of the adhesive. Since considerable shrinkage took place in the large volume of joint space, the tape covering the joint had to be placed over the joint in such a manner that the cast adhesive would develop an external bead over the joint. To form this bead, the tape was partially folded over the joint and, since the heat of the reaction in the cast adhesive may change the shape of the fold in the tape, it was stiffened locally in the fold area by aluminum foil. This aluminum foil provided a further advantage by giving the joint bead a smooth surface.

The troubles, however, did not terminate with the placement and setting of adhesive in the joint spaces, because small dispersed cavities (Figure 51) and separation spaces (Figure 52) were found in the joint after it had set. The separation spaces generally were found at the boundaries between individual pours. Only after a brief investigation into methods of eliminating them was it found that the removal of the surface skin from the preceding already set pour would in most cases eliminate the separation spaces between neighboring PS-18 pours in the joint. Separation spaces 
that were already present in the joints because the surface of the preceding pour was not removed were eliminated in some cases by drilling or milling out (trepanning) the separation space and refilling it with fresh PS-18. No reliable method was found to prevent formation of small dispersed cavities in the set adhesive, although it can be hypothesized that a too-rapid setting rate (triggered by high ambient atmospheric temperature) was probably their cause. This hypothesis is substantiated by the fact that some of the pours resulted in completely cavity-free joints, while others had many cavities even though the chemical composition of the mix was identical in all cases. Most of the cavities were too small to merit a trepanning operation, particularly since it was felt that in joints subjected to operational tensile stresses less than 200 psi, the debilitating effect of cavities on the 4,000-to-5,000-psi ability of the joint to withstand 200-psi stresses was negligible. Only in very few cases where the cavities appeared to be interconnected was a trepanning operation performed and the cut refilled with fresh PS-18 adhesive. The trepanning and refilling of separation spaces in the joint, however, generally did not decrease the peak residual stress level at that location, and in most cases introduced many incipient fine cracks (Figures 53 and B-2b). For this reason, it was used only when the separation space penetrated the whole joint width creating a leak (three locations).

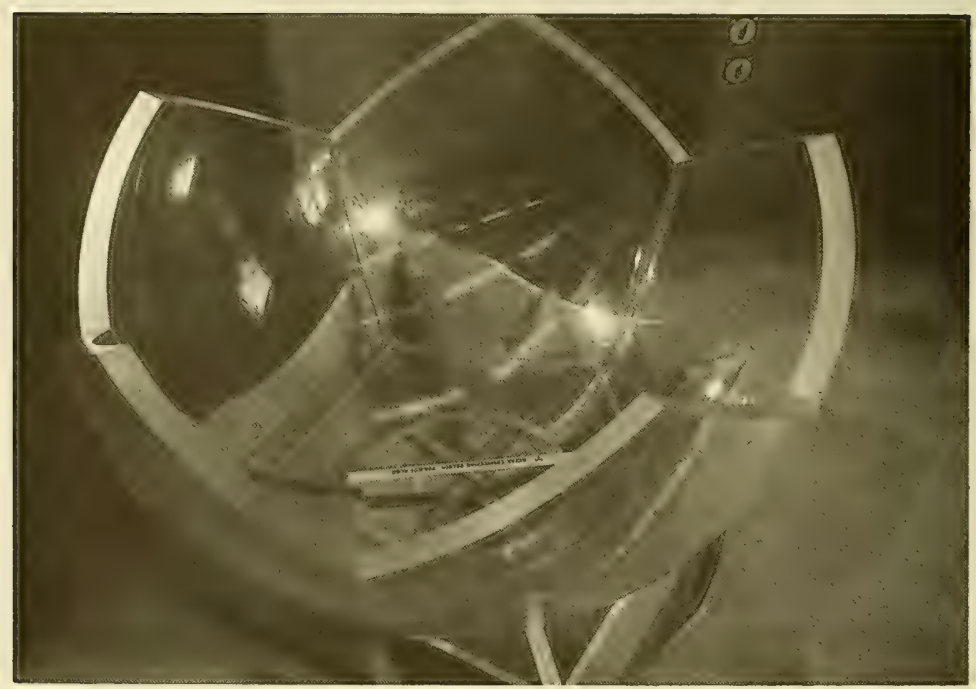

Figure 49. Bonded hemisphere after removal from assembly jig. 


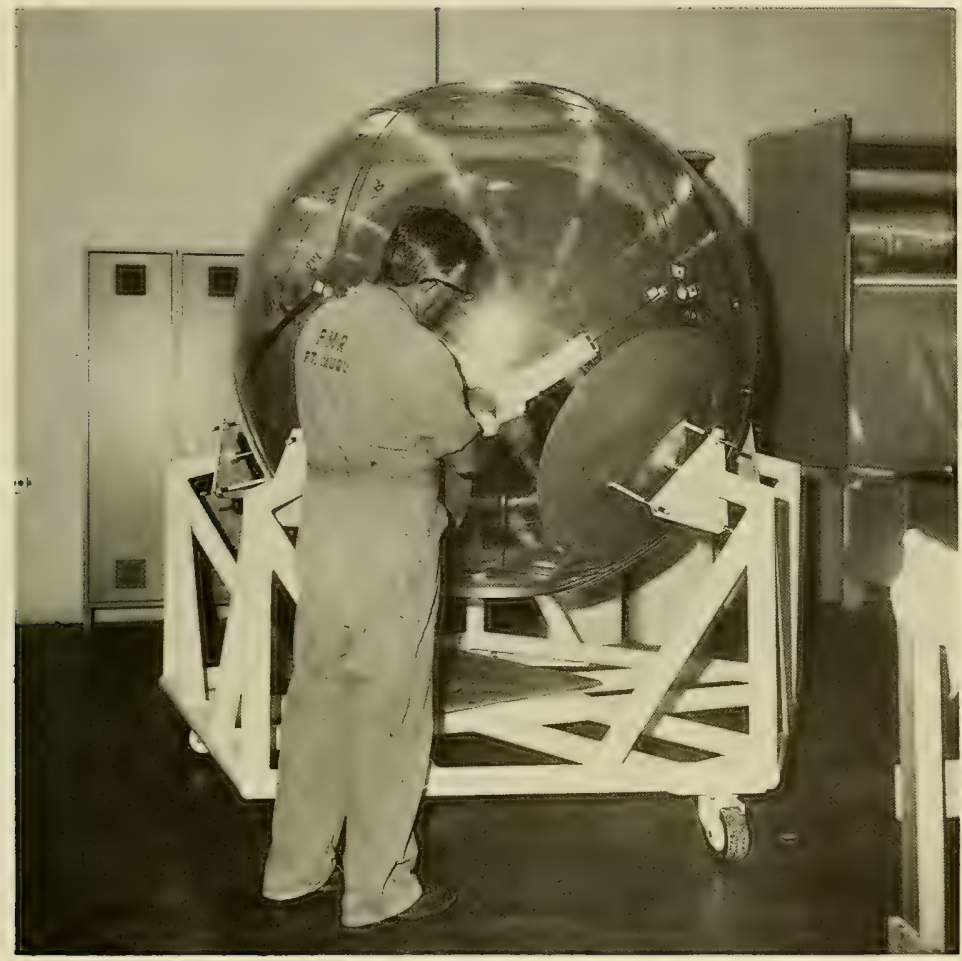

Figure 50. Preparing the two hemispheres for bonding together into a sphere.

Because of all the problems encountered during the bonding operations, the resulting joints in the 66-inch prototype capsule leave much to be desired and constitute a fruitful area for future improvement. However, one very valuable finding about joints could be expected from hydrostatic testing of the prototype large-scale capsule: Successful, structural integrity of the hull and joints under both operational and proof pressures would serve as proof that the presence of discontinuities (dispersed cavities and separation spaces) in the joints is not a bar to safe operational performance of a spherical acrylic plastic capsule under external hydrostatic loading. On the other hand, if the cavities and separation spaces served as fatigue crack initiators leading to early cyclic fatigue failure, future joints would have to be cast utilizing more refined casting techniques and under tighter quality control to prevent the occurrence of any cavities. 


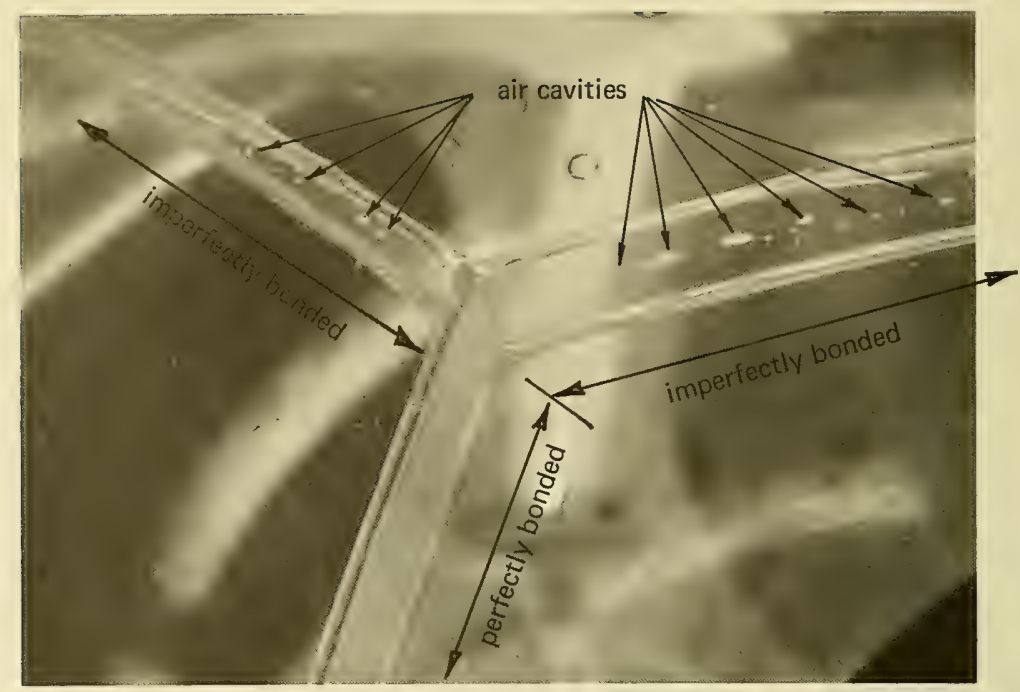

Figure 51. Typical cavities in an imperfectly bonded segment of a joint in 66-inch-diameter capsule.

After completion of bonding, the large-scale acrylic plastic hull was annealed for 124 hours at $160^{\circ} \mathrm{F}$ in a temperature-controlled oven. The annealing process did not remove all the residual stresses from the joints resulting from the bonding process. The residual stresses in the joints were found to be approximately of the same order of magnitude (but somewhat less) as the residual stresses in the model capsule (Figure 54). The basic difference between the residual stresses in the model capsule and the largescale capsule was in distribution. In the model capsule, the stresses are fairly uniform in all of the joints, but in the large-scale capsule the magnitude of stresses varies markedly from one location to another in the joint. This, of course, is the result of bonding the joints in the large-scale capsule in small increments, each solidifying before the next one is cast. Thus, it appears that casting of joints in small increments is less desirable than casting them in one or two increments, or preferably in a single operation.

Polishing of the exterior and interior of the hull completed the fabrication of the large-scale acrylic plastic hull. Sphericity and diametral measurements revealed that zealous polishing of rough joints removed an excessive amount of acrylic plastic. The lesson that could be drawn from this is that polishing should be performed with great care, and if the choice 
exists of removing an excessive amount of acrylic plastic versus having some roughness around joint mismatches, it is better to leave that roughness. Also, extreme care must be exercised not to exert so much pressure on the buffing wheel that local heating and galling of the acrylic plastic occurs. Since this care was not exercised at all times during buffing of the large-scale capsule, some surface areas on the acrylic plastic hull were locally scuffed.

Dimensional Inspection. At the completion of fabrication, each individual scale model was checked for its sphericity and diameter. This dimensional check together with previously conducted sphericity and thickness measurements of individual pentagons constituted the complete record for an individual NEMO model. Since the paperwork representing the complete dimensional record of each model NEMO is quite voluminous, only the dimensional record for one typical NEMO model has been included in this report (Appendix C). This dimensional record is briefly summarized here:

$\begin{array}{lccc} & \text { NEMO Model 18 } & & \text { Nominal } \\ & & \\ \text { Hull Thickness (in.) } & 0.515-0.465 & & 0.500 \\ \text { Internal Radius (in.) } & 7.028-6.994 & 7.000 \\ \text { Outside Diameter (in.) } & 15.040-14.995 & & 15.000\end{array}$

These deviations from the nominal can be considered representative for all the other scale models with one or two exceptions. One of the models, NEMO no. 1, had sphericity deviations considerably in excess of the representative values enumerated above. The internal sphericity and thickness of this model varied from 7.200 to 6.875 inches and from 0.495 to 0.450 inch, respectively. Although those sphericity deviations were considered unacceptable $(1,000 \%$ larger than the permissible deviation of $0.03 t$ ) the NEMO Model no. 1 was used in the test program to determine the effect of excessive sphericity deviations on the collapse pressure of acrylic plastic hulls under long-term hydrostatic loading.

The measurement of thickness and sphericity on individual pentagons was performed utilizing micrometers and a dial gage mounted in a vertical mill. Upon assembly of pentagons into a sphere, the sphericity was measured with a female template and feeler gage while the diameter was measured with dialindicator-equipped calipers. 


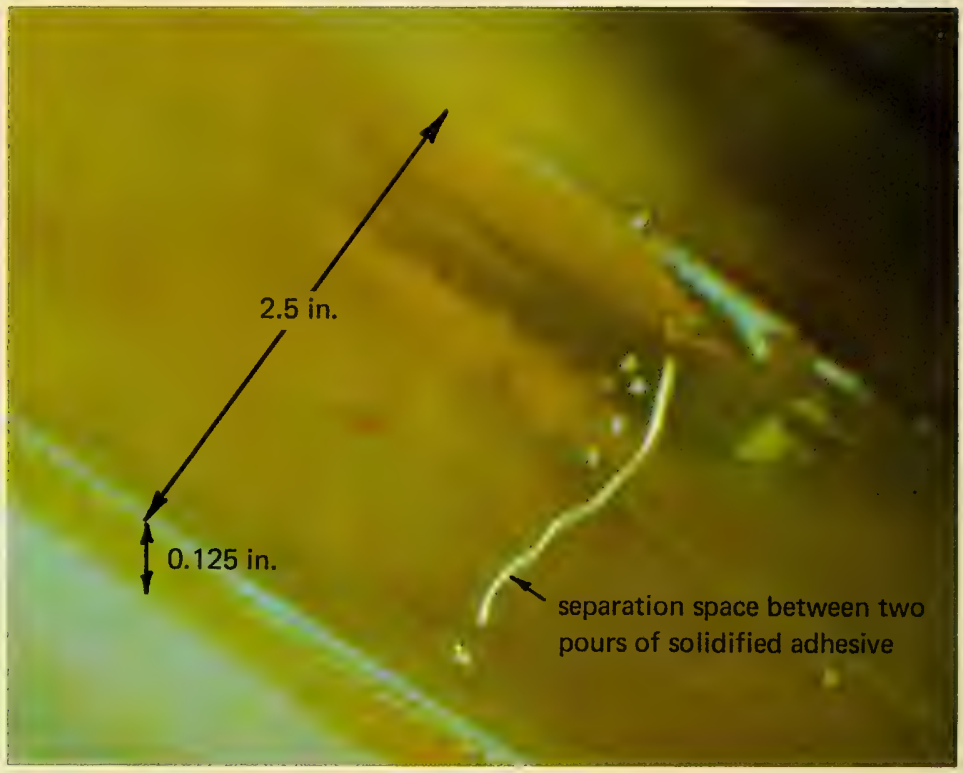

Figure 52. Typical separation space in an improperly bonded segment of the joint in the 66-inch-diameter capsule.

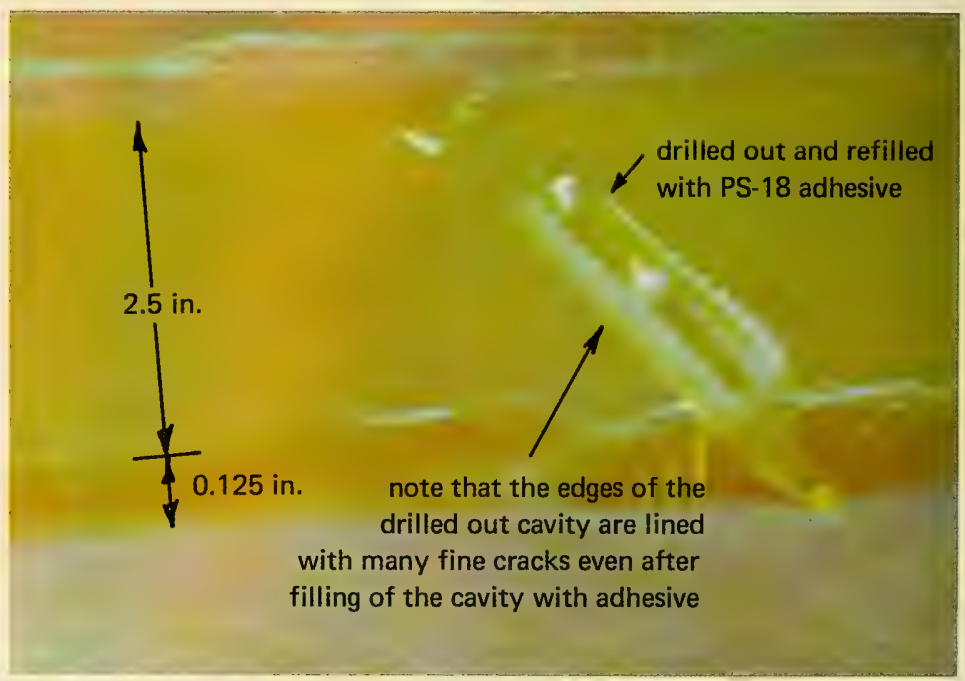

Figure 53. Results of a local trepanning operation in the joint of the 66-inchdiameter capsule. 


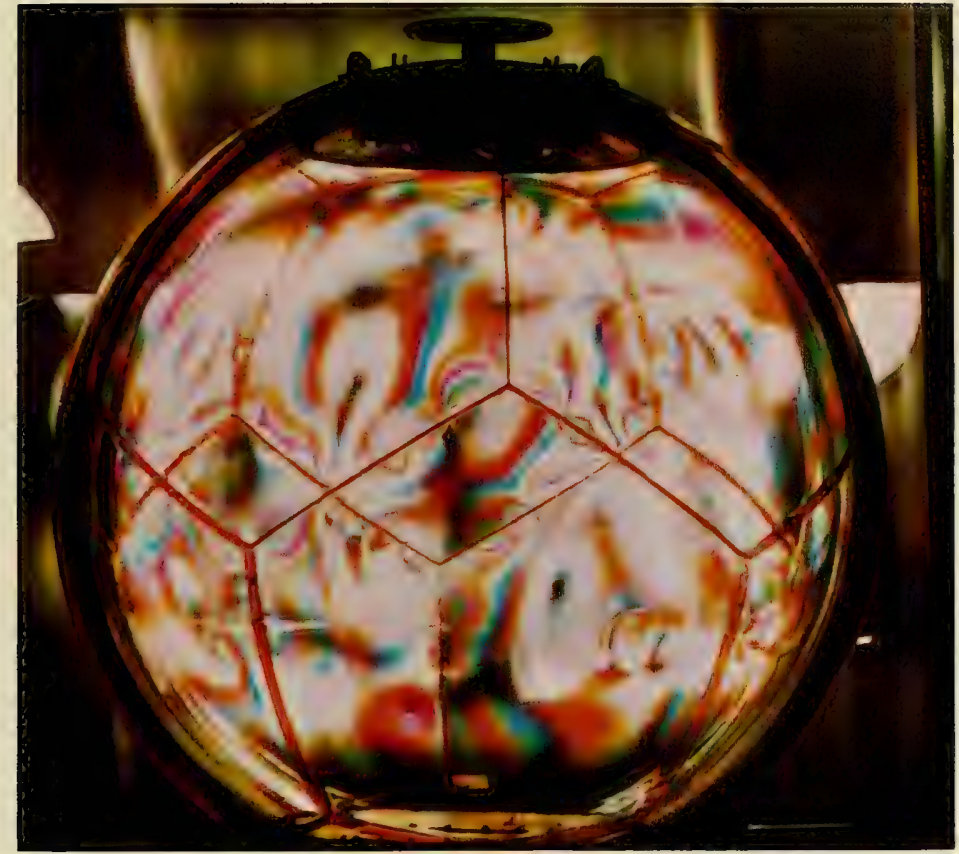

(a) Overview.

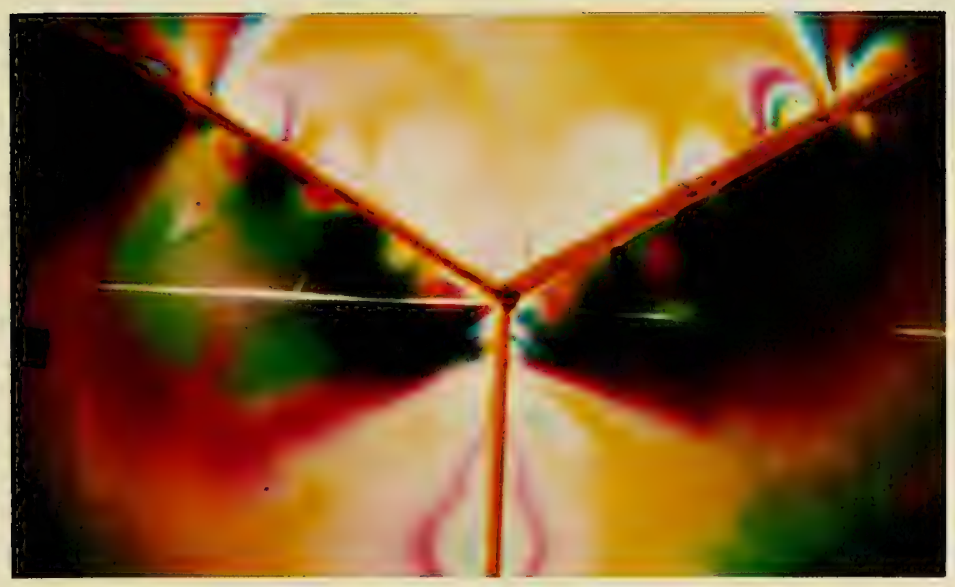

(b) Detail.

Figure 54. Residual stresses in the 66-inch-diameter capsule not removed by the annealing operation. 


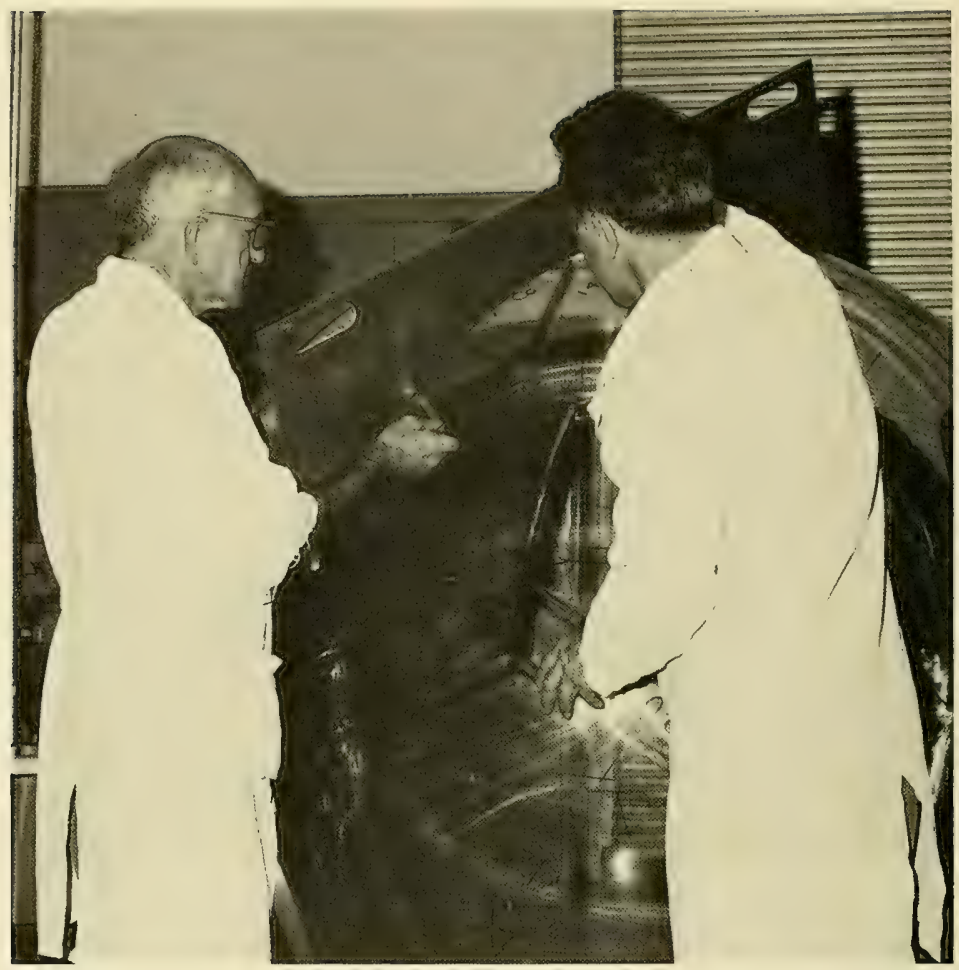

Figure 55. Measuring the sphericity of the bonded 66-inch-diameter capsule with a template having a nominal radius of 33 inches.

The large-scale acrylic plastic capsule was dimensionally checked (Figures 55 and 56) in the same manner as the scale models by using a template, dial gage, feeler gages, and caliper mounted micrometers. All the dimensional control data taken on individual pentagons prior to assembly, and subsequently after bonding and annealing of the large-scale acrylic plastic hull has been included in this report (Appendix $A$ ) to serve as a standard of comparison for (1) future operational large-scale NEMO hulls and (2) show the effect scaling up models has on dimensional deviations. The dimensional control data of the large-scale NEMO hull can be summarized here by stating that the wall thickness and internal sphericity of individual pentagons prior 
to assembly varied from 2.285 to 2.585 inches and 30.702 to 30.345 inches, respectively, while the external sphericity and the diameter of the assembled capsule varied from 33.134 to 33.000 inches and 66.158 to 65.920 inches, respectively. The difference between sphericity measurements taken on individual pentagons and on the finished hull are attributed in a large measure to (1) mismatch between individual pentagons during assembly, (2) excessive polishing and buffing of the joint surface areas, and (3) difference in measuring techniques (in one case dial indicator measurement of internal radius while in the other case template measurement of the external radius).

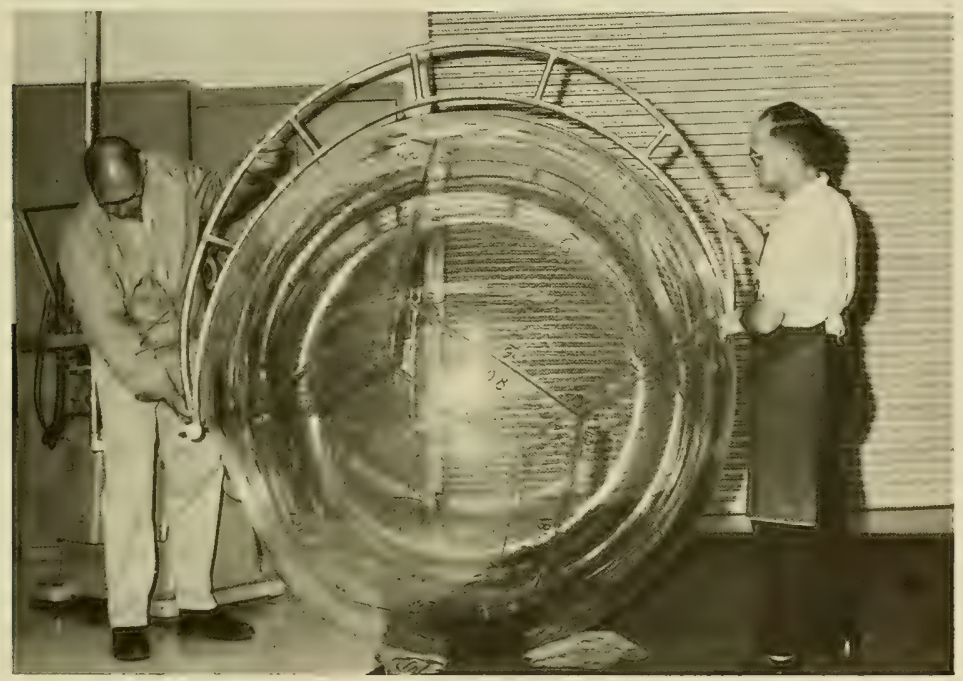

Figure 56. Measuring the diametral deviations of the bonded 66-inch capsule with a 66-inch-throat micrometer.

The magnitudes of sphericity, thickness, and diametral deviations from specified nominal values when related to the size of the large-scale capsule show that these deviations are approximately of the same magnitude as those measured on model capsules. While the relative sphericity deviation from the specified internal radius on scale models was within +0.09 and $-0.4 \%$, on the large-scale acrylic plastic hull it was within +0.50 and $-0.66 \%$. The relative diametral deviation from nominal diameter for scale models was within +0.26 and $-0.03 \%$, while the large-scale hull's diametral deviations were within +0.25 and $-0.12 \%$. Since the comparison of relative dimensional deviations has 
shown that the large-scale hull presents approximately as perfect a sphere as the models, any data generated by testing of small-scale models should be considered as applicable to the prediction of large-scale capsule performance. Also, it can be hypothesized that scaling up acrylic plastic spherical capsule models results in large spheres with approximately the same relative dimensional deviations, providing the same fabrication method is used for model and large-scale capsules.

Before the discussion of relative dimensional tolerances is concluded, a point brought up in the discussion of the dimensional relationship between model and large-scale capsules should be reemphasized: Since in the fabrication of models it was found that the actual thickness of the hull consistently was less than specified, it was decided to order oversize material for the large-scale capsule so that even with the large tolerances on thickness of stock materials the hull thickness would not be below specified dimensions. Thus, 2.500-inch stock material was used in large-scale capsule, although 2.250-inch nominal thickness is actually required on the basis of geometrical scaling. Because of this, even though the hull thickness varied from 2.290 to 2.585 inches, it was always above the nominal thickness. This supports the author's assurance that all the experimental data recorded on models is not only applicable, but even probably conservative since the models were actually 10 to 20\% thinner than their nominal thickness arrived at by scaling down the actual large-scale capsule thickness.

\section{Polar Inserts}

The steel structural components required for the assembly of top and bottom polar inserts for the model scale capsules were fabricated by turning 316 stainless steel bar stock in a lathe. Particular emphasis was placed on adherence to the tight angular and dimensional tolerances on the beveled bearing and sealing surfaces on the hatch ring, hatch and bottom plate.

Since turning these components for the capsule was a typical metal machining operation, no research or development was necessary during their fabrication.

No problems were encountered in the fabrication of metallic inserts for the 66-inch capsule. The only basic difference between fabrication of the inserts for models and of the 66-inch capsule was the use of type 316L stainless steel and the welding of lifting lugs and bosses on the top hatch assembly instead of milling them out of a solid forging. To make the fabrication economical, the hatch ring, hatch plate, and bottom plate were ordered from suppliers as slightly oversized forgings for machining. 


\section{PHASES 3 AND 4-EVALUATION OF DESIGN AND FABRICATION}

\section{Capsule Test Specimens}

The model capsule series consisted of 22 acrylic plastic hulls with nominal 15-inch outside diameter and 14-inch inside diameter. All were made of one-half-inch grade G Plexiglas plate stock that had been cut, thermoformed, and machined into spherical pentagons that subsequently were assembled and bonded with acrylic adhesives. The details of forming, machining, and bonding were discussed under "Fabrication Process." It is important to note here that models no. 1 through 11 were bonded with solvent-type adhesive while those numbered 11 through 22 were bonded with the self-polymerizing PS-18 cement.

Three distinctly different types of metallic polar plates were fabricated for the model NEMO series.

1. Operational polar plates represented faithfully scaled-down hatches and bottom plates proposed for the 120-inch capsule (Figures 19 and 57 through 62). All attempts were made here to reproduce all the working parts and details of the end plates that would be found in the 120-inch NEMO. The top hatch not only had a hatch that opened, but also the locking mechanism required for it. Since two different hatch designs (Figures 19 and 60) were proposed for the 120-inch capsule, two faithfully scaled-down hatch designs were also fabricated for the model capsules. The bottom plate in the operational end plates (Figure 18) incorporated all the penetrations planned for the large capsule. It was felt that if the operational polar steel inserts performed successfully on models without any difficulties (jamming of hatches, failure of electrical penetrators, pulling out of end plates under hoisting operations, or ripping out of retention flanges under hydrostatic loading), no unforeseen difficulties would be encountered during the operation of the proposed 120-inch NEMO system or any scaleddown version of it (for example, the 66-inch system).

Some of the models equipped with operational end plates had also internal tie rods connecting the top hatch with the bottom plate. The purpose of these pretensioned tie rods was to subject the acrylic plastic capsule to compressive preload while the capsule was out of water. Use of tie rods on some of the models was considered necessary to simulate the 120 -inch NEMO that probably would utilize internal tie rods for reduction of tensile stresses in the sphere when it floats on sea surface. For the models, each of the four tie rods was preloaded 25 pounds. Together, they applied sufficient force on the polar plates to counteract the forces that would be exerted 
upon the end plates by the internally or externally located equipment when the capsule was lowered on the sea surface. The spring assemblies (Figure 23) on the tie rods were of such design that when the capsule was submerged to its design depth of 1,000 feet and remained on station for 100 hours, the diametral decrease of the hull would match the original extension of the springs and thus the total tie-rod preload would decrease to zero. Upon depressurization of the capsule, the dilation of the hull would contract the springs again and the tie-rod system would again be under the specified preload of 100 pounds.

Some models were not equipped with internal tie rods in order to (1) simulate NEMO systems that would not utilize tie rods and (2) to compare experimentally the effect of preloaded tie rods on the critical pressure of the acrylic hulls.

All models equipped with operational polar plates were ballasted with properly distributed weights simulating the weight distribution on the 120-inch-diameter NEMO. A portion of the weights rested on the bottom plate simulating the weight of the crew, controls, and life support, while a sufficiently large weight was suspended from the bottom plate to give the whole model negative buoyancy (Figure 57).

2. Simulated end plates were structurally similar to the operational end plates but had none of the structural details or working parts found in the operational end plates (Figure 63). The simulated end plates were used on the majority of models (Figure 64) for economy. Since, however, they like the operational end plates were designed to fail in the 1,300-to-1,600-psi pressure range as were the acrylic plastic hulls, they were generally not employed in the model tests at low temperatures where the implosions were predicted to take place at pressures in the 2,000-psi range.

3. Heavy-duty end plates were simply metallic end plugs of the same thickness and curvature as the acrylic plastic hull closing off the polar openings in the acrylic plastic hull (Figure 65). The heavy-duty end plates were fabricated from 6AL-4Va titanium alloy to give them high strength while at the same time keeping their rigidity low so that the mismatch between the rigidities of the end plate and the hull did not generate undue stress concentrations in the acrylic plastic. The heavy-duty end plates were used only in those capsule models that were to be tested at low ambient temperatures that could increase the critical pressure of the acrylic plastic hulis above the design pressure of the operational or the simulated hatches of 316 stainless steel. 


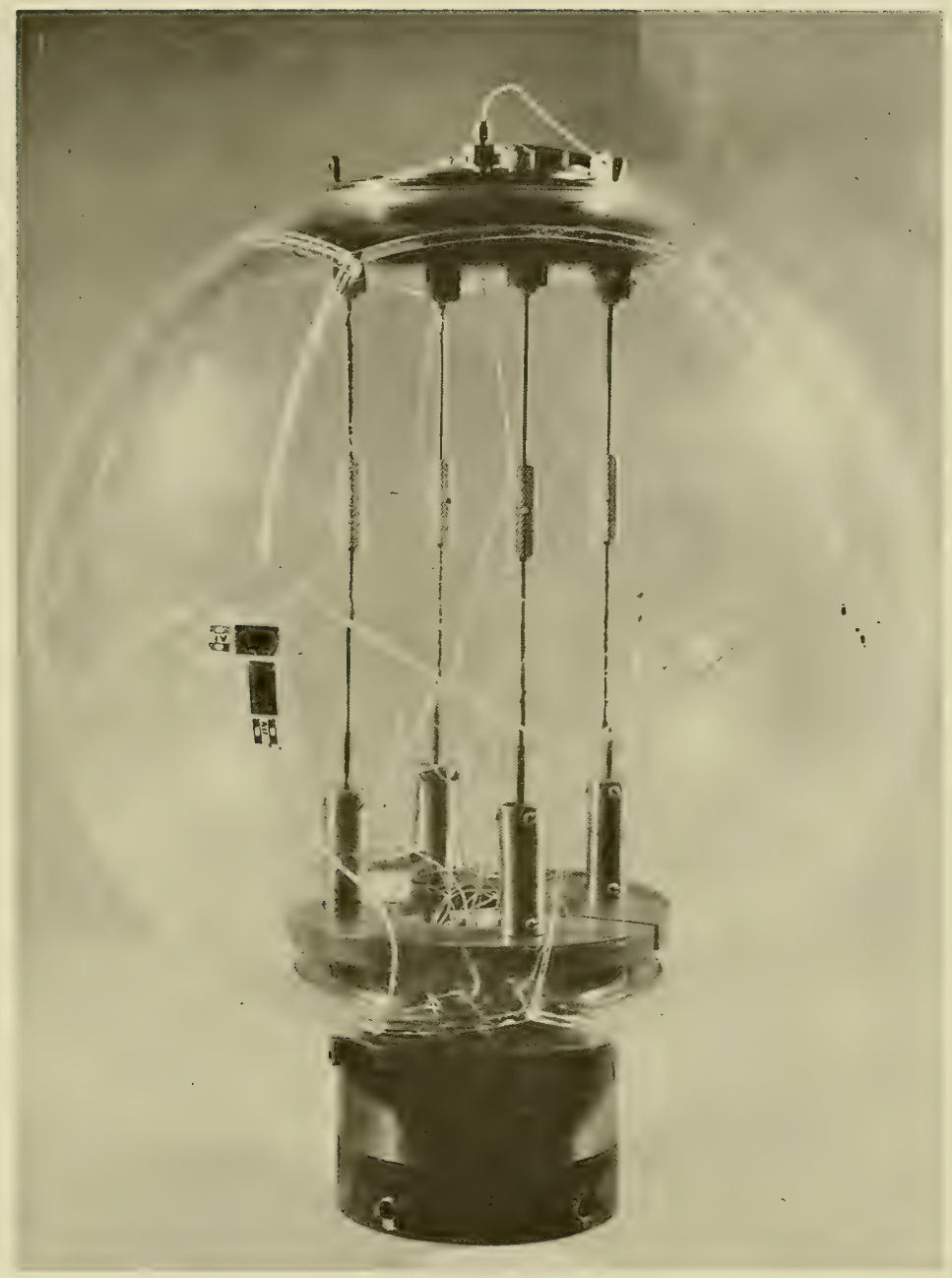

Figure 57. Operational 15-inch-diameter model acrylic plastic capsule for the NEMO system: assembled. 


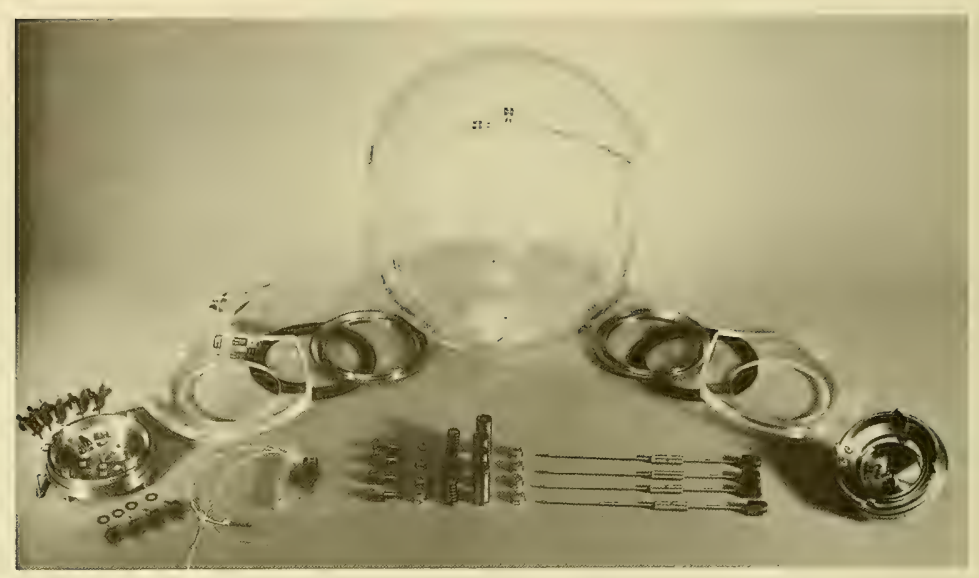

Figure 58. Operational 15-inch-diameter model acrylic plastic capsule for NEMO system: disassembled.

The large-scale test series consisted of a single 66-inch-OD and 61-inch-ID acrylic plastic hull equipped with operational end plates of type 316 stainless steel (Figure 66). The hull, as previously described under "Fabrication Process," was fabricated in much the same way as the model hulls. The bottom plate was a faithfully scaled-up copy of the operational bottom plate in the model NEMO with the exception of the internal retaining flange, which was bolted to the bottom plate instead of being threaded to it (Figure 67). The top hatch was also a scaled-up version of the operational hatch used in the NEMO models (Figure 68). The only difference between it and the working hatches in the models were the use of a bolted-on, instead of screwed-on, retaining flange (Figure 67) and the use of a planetary gear system, instead of a cam system, for the locking and unlocking mechanism. (For detailed dimensions, see Appendix A.) Pretensioned tie rods were not used as (1) there would not be sufficient room for them in a 66-inch capsule for manned operations and (2) absence of the rods subjected the hull to a more severe test condition in the pressure vessel than the capsule would see in actual service, where the external cage would resist the downward pull of external ballast.

\section{Testing Program for Capsules}

The capsule test specimens were tested for their structural integrity mostly by external hydrostatic loading. This type of loading was selected because it represents (1) the most severe loading condition to which the structure will be subjected in the ocean and (2) it is the loading condition 
to which the structure will be subjected the largest percentage of time during its operational life. Since the test fixtures and test details associated with the hydrostatic testing of capsule specimens varied to some extent between the model and large-scale specimen tests, they will be described separately.

Testing of Models. Fifteen-inch capsule models were subjected to a large variety of hydrostatic tests in order to provide experimental data on as many facets of capsules' structural response to hydrostatic loading as possible. The hydrostatic pressure tests to which the model capsules were subjected can be divided into three general categories: (1) short-term tests, (2) longterm tests, and (3) cyclic tests. In addition to the hydrostatic tests, a single axial compression test was also performed.

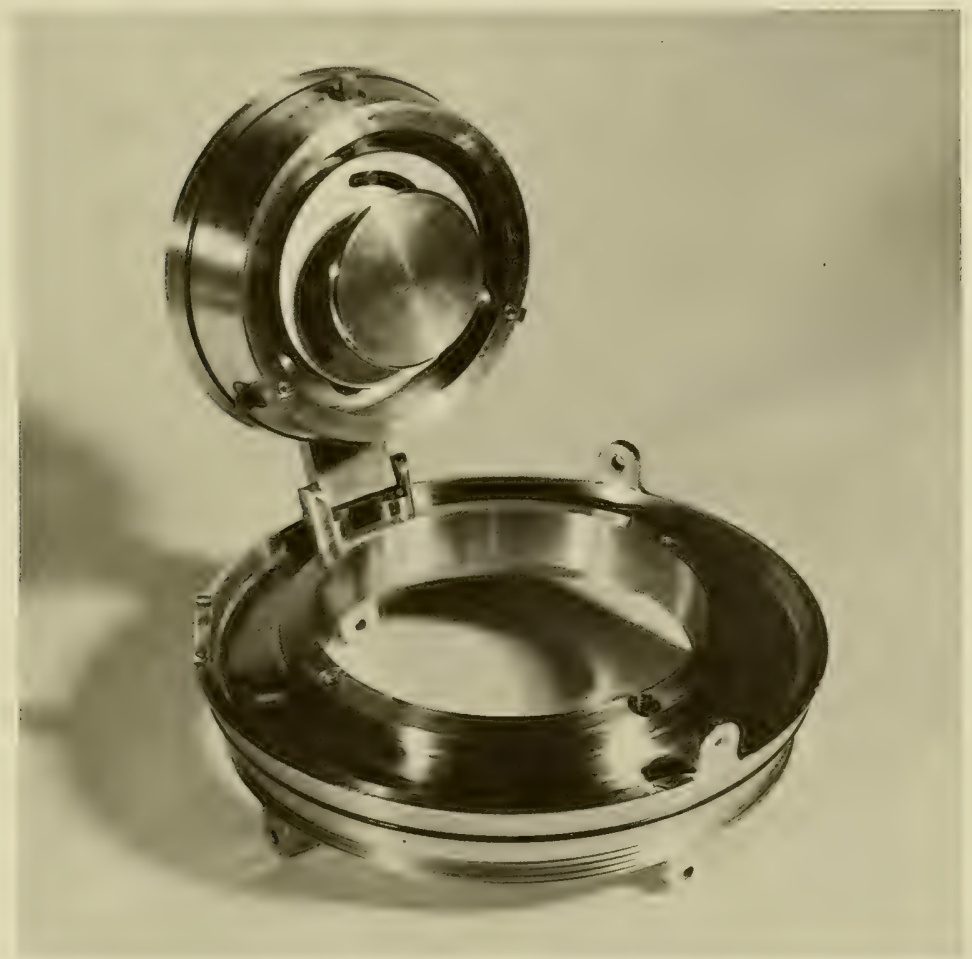

Figure 59. Hatch for the operational 15-inch-diameter model capsule: open. 


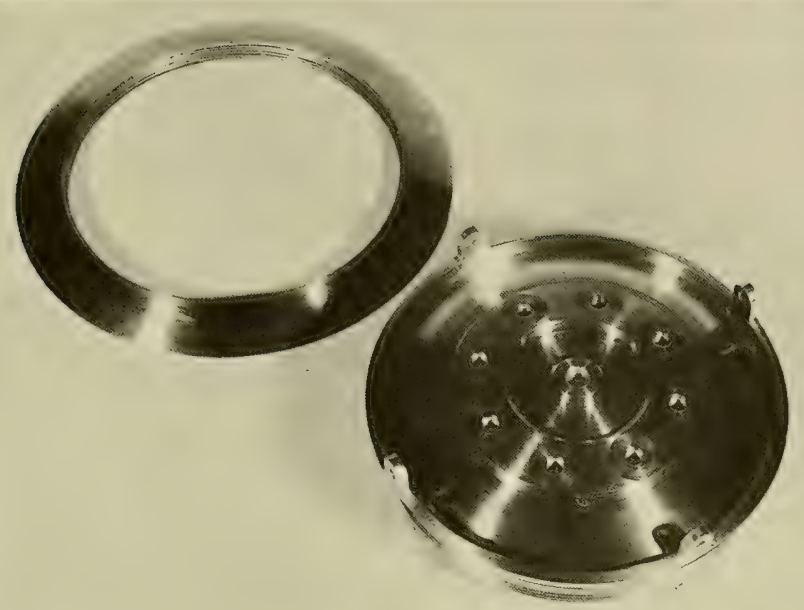

Figure 61. Bottom polar penetration closure and retaining ring for the 15-inchdiameter model capsule: exterior view.

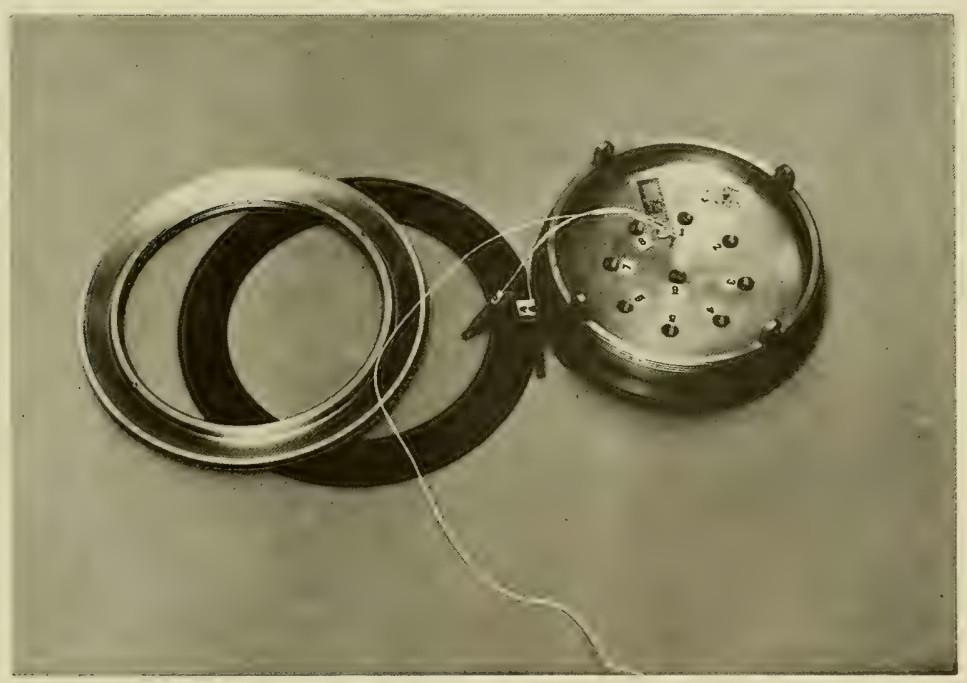

Figure 62. Bottom polar penetration closure and retaining ring for the 15-inchdiameter model capsule: interior view. 


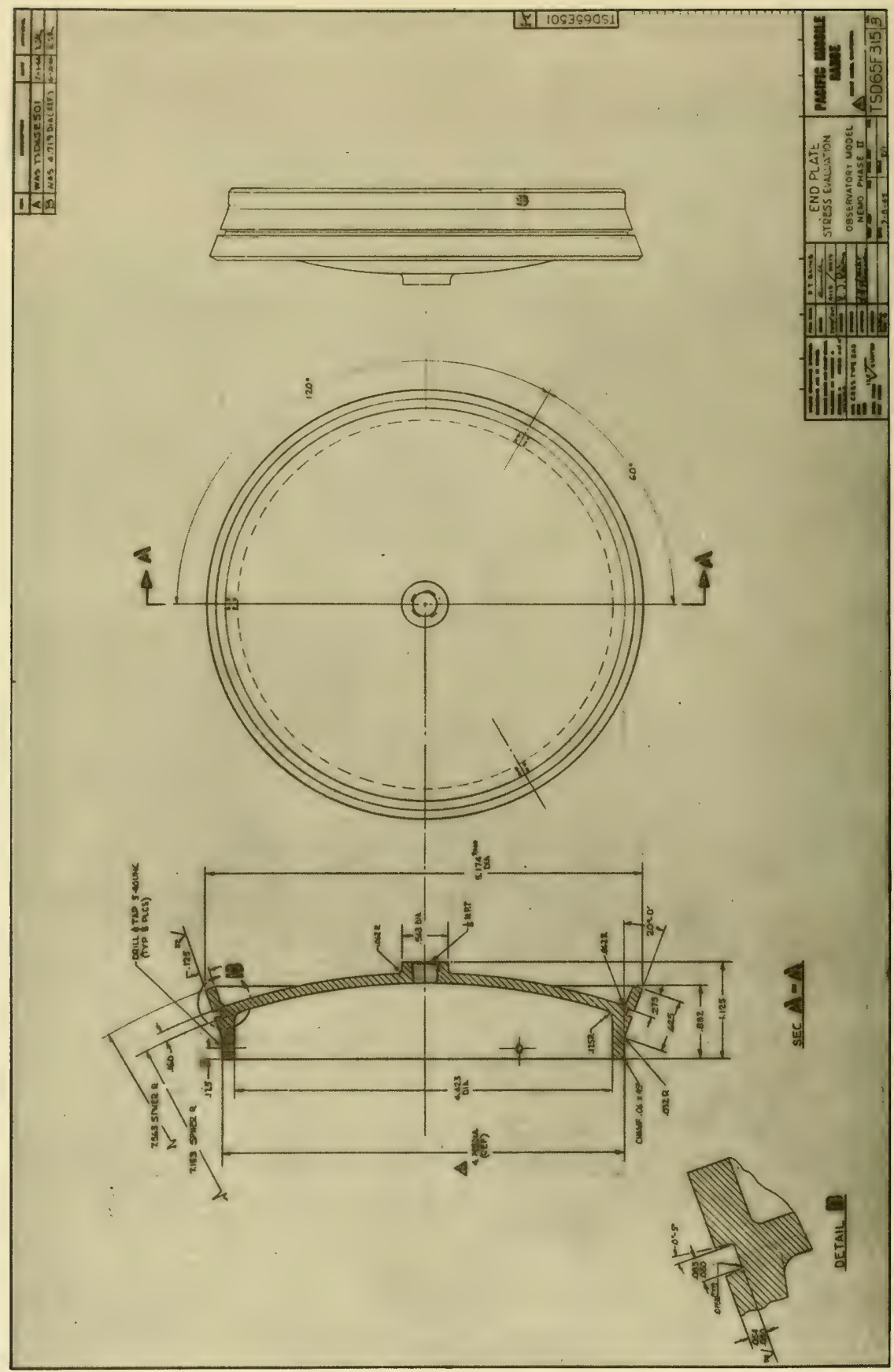

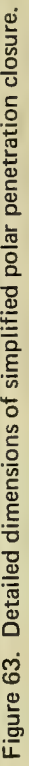




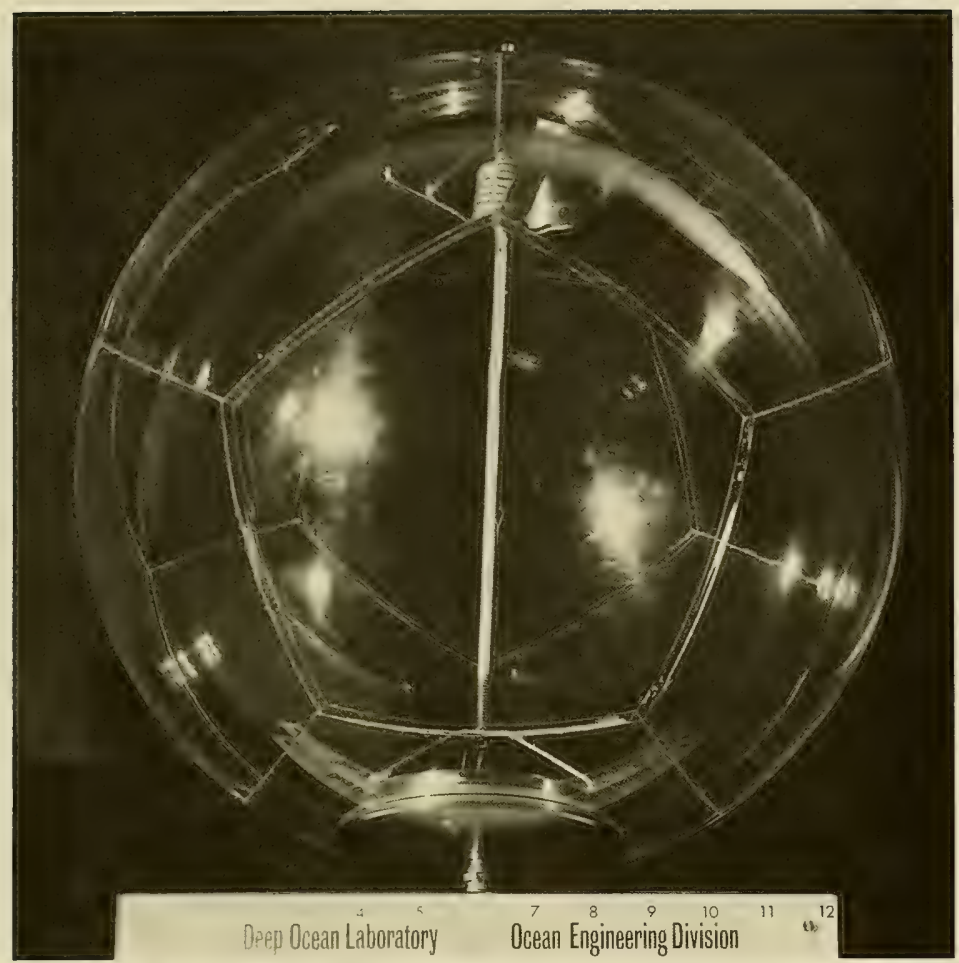

Figure 64. Model capsule of 15-inch diameter with simplified polar penetration closures: assembly. Note the single spring-loaded tie rod.

Short-term tests were performed on 11 capsules (Table 4). All of the models were equipped with tie rods except for capsules 0,16 and 20 . These tests were to provide immediate data on the maximum hydrostatic pressure the capsule can withstand under different temperatures in the 32 to $114^{\circ} \mathrm{F}$ range. Furthermore, the critical pressures at which implosion of the models occurred would serve as benchmarks to which critical pressures of models tested under long term (Table 5) and cyclic (Table 6) conditions could be compared to determine the effect of cycling, or long-term loading on their structural integrity. For all of these tests, water was used on the exterior and interior to insure proper temperature control. Since the water in the interior of the hull was vented to the atmosphere by tubing, its presence had no reinforcing effect on the hull. 


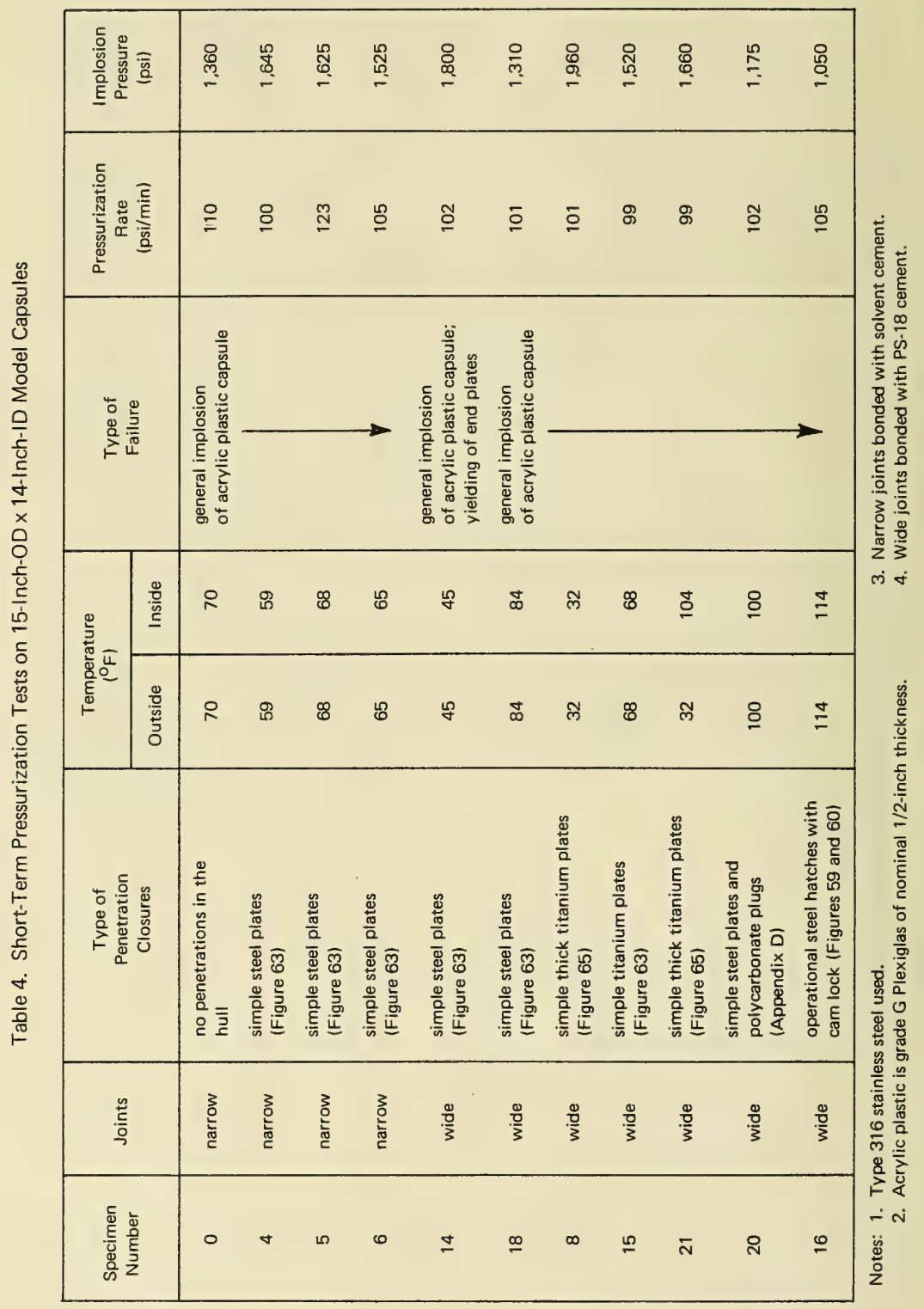




\begin{tabular}{|c|c|c|c|c|c|c|c|c|c|c|c|}
\hline \multicolumn{2}{|c|}{ 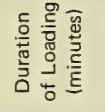 } & $\omega$ & 요 & 옴 & ஜ్లి & 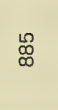 & 号 & 㣽 & 怘 & 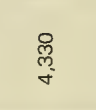 & $\begin{array}{l}\text { ㅇ } \\
8 \\
\text { 유 }\end{array}$ \\
\hline \multicolumn{2}{|c|}{ 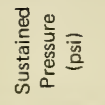 } & ঃ্ & 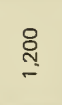 & $\stackrel{8}{\circ}$ & $\stackrel{8}{8}$ & \& & $\stackrel{8}{8}$ & 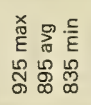 & 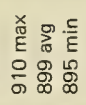 & 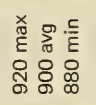 & 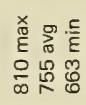 \\
\hline \multicolumn{2}{|c|}{ 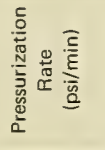 } & ণ̃ & $\stackrel{\bullet}{\circ}$ & 음 & ํㅡㅁ & $\stackrel{10}{\circ}$ & $\stackrel{ }{=}$ & $\stackrel{8}{\circ}$ & $\nsubseteq$ & $\stackrel{\text { ஜ }}{\circ}$ & $\underline{0}$ \\
\hline \multicolumn{2}{|c|}{ 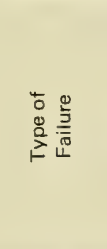 } & 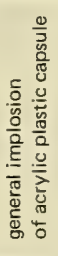 & & & & & & & & & 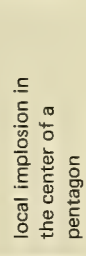 \\
\hline \multirow{2}{*}{ 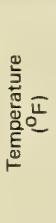 } & 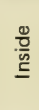 & $\ddot{0}$ & $\ddot{8}$ & $\ddot{्}$ & $\mathscr{8}$ & $\notin$ & $\ddot{8}$ & R & छే & 8 & 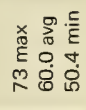 \\
\hline & $\frac{0}{0}$ & $\mathscr{0}$ & $\mathscr{B}$ & ஜ8 & $\ddot{8}$ & 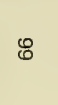 & $\ddot{\otimes}$ & $\stackrel{\circ}{\curvearrowright}$ & छే & $\ddot{8}$ & 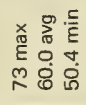 \\
\hline \multicolumn{2}{|c|}{ 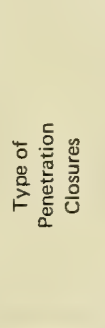 } & 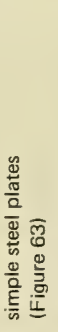 & 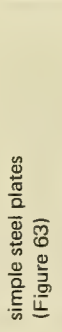 & 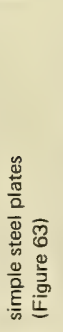 & 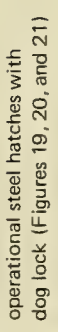 & 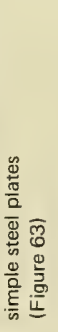 & 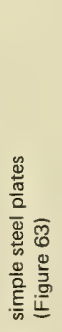 & 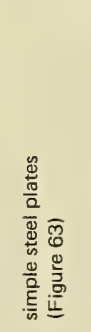 & 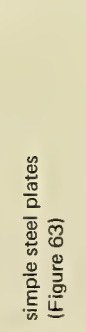 & 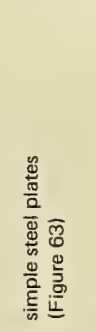 & 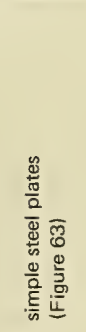 \\
\hline \multicolumn{2}{|c|}{. } & క气 & $\frac{3}{0}$ & $\frac{0}{3}$ & 3ั & ב⿱ & 豙 & $\frac{0}{3}$ & $\frac{0}{3}$ & 胥 & $\begin{array}{l}3 \\
\text { 总 } \\
\text { ¿ }\end{array}$ \\
\hline \multicolumn{2}{|c|}{ 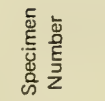 } & $=$ & $\stackrel{\square}{-}$ & $=$ & - & 으 & r & $\stackrel{\sim}{\sim}$ & $\stackrel{m}{-}$ & a & m \\
\hline
\end{tabular}




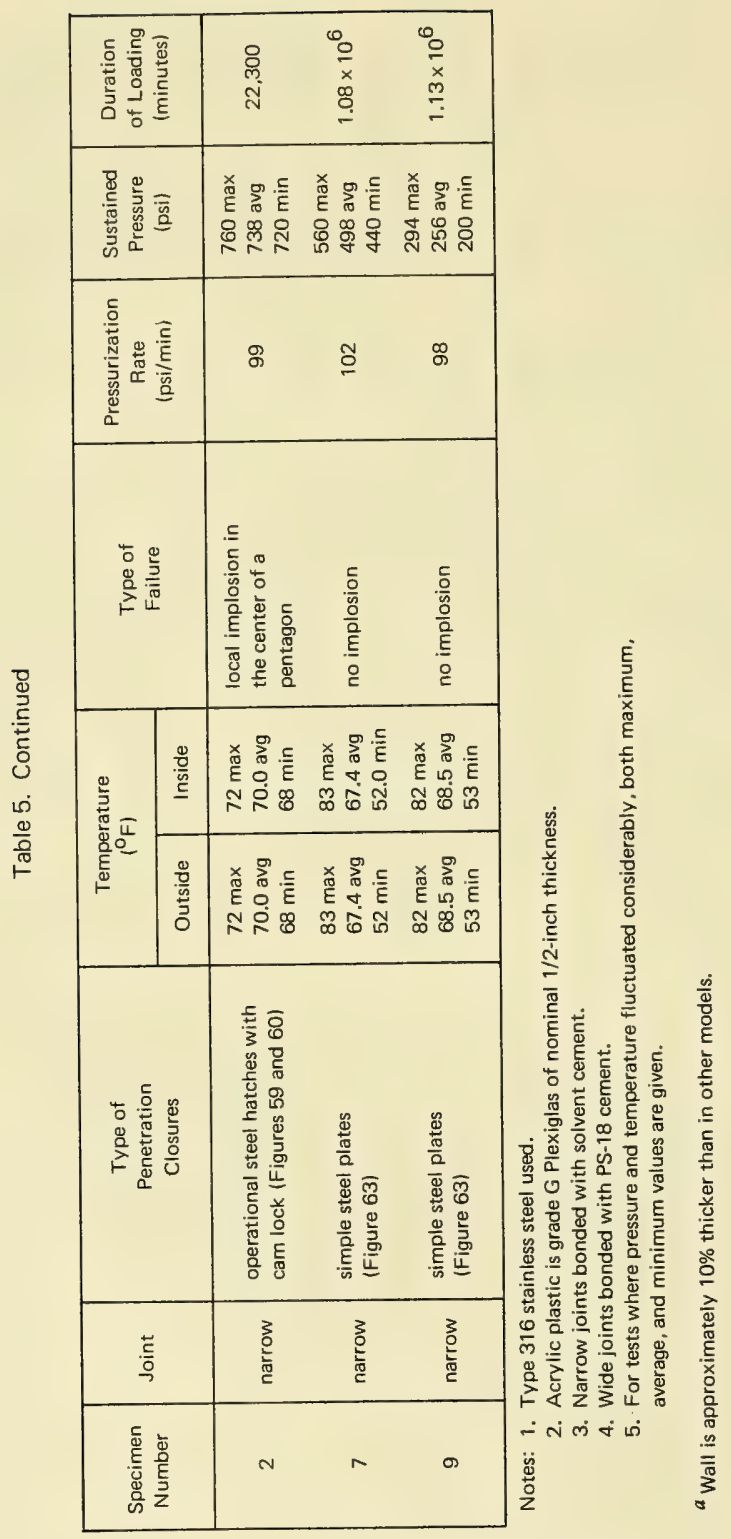




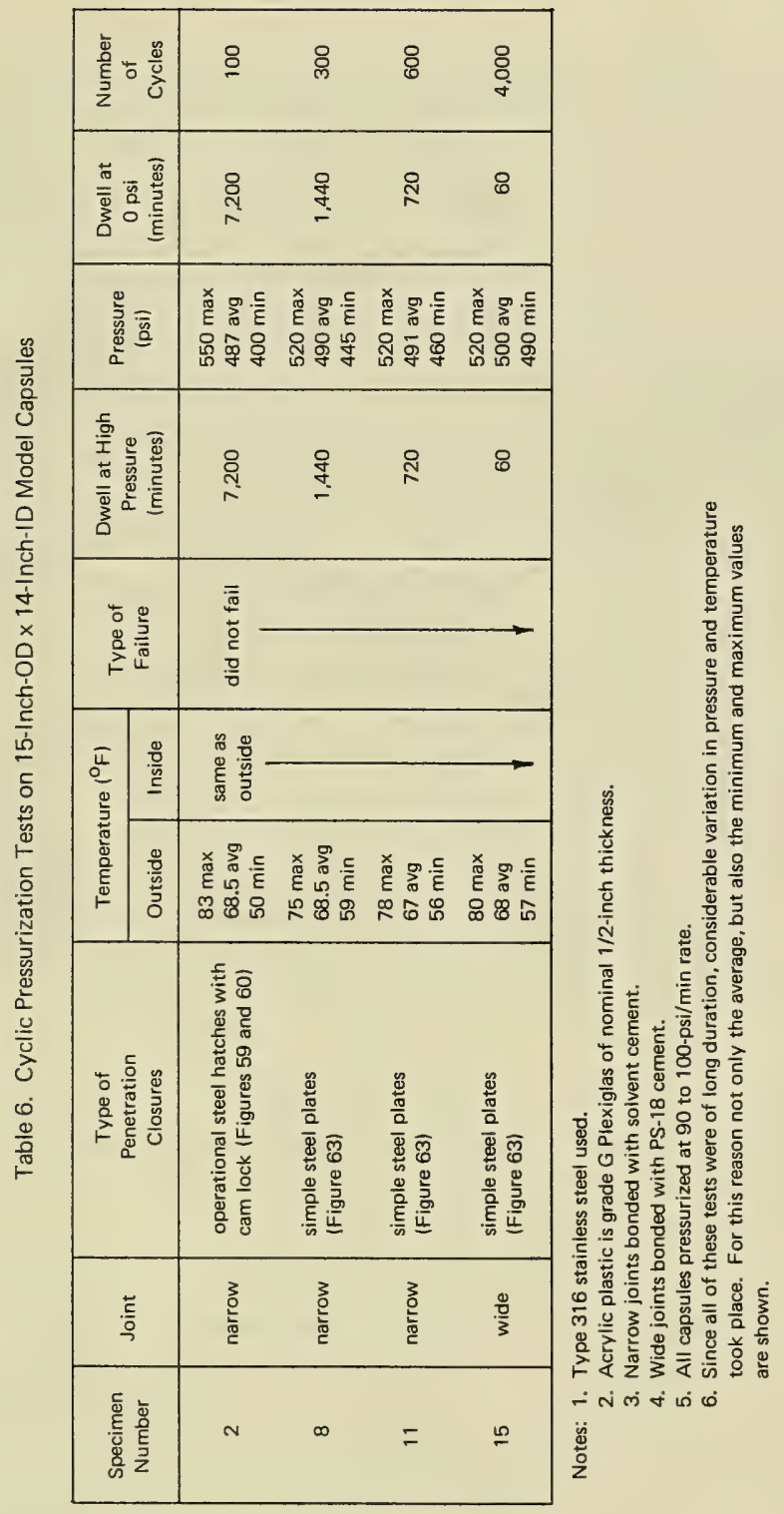




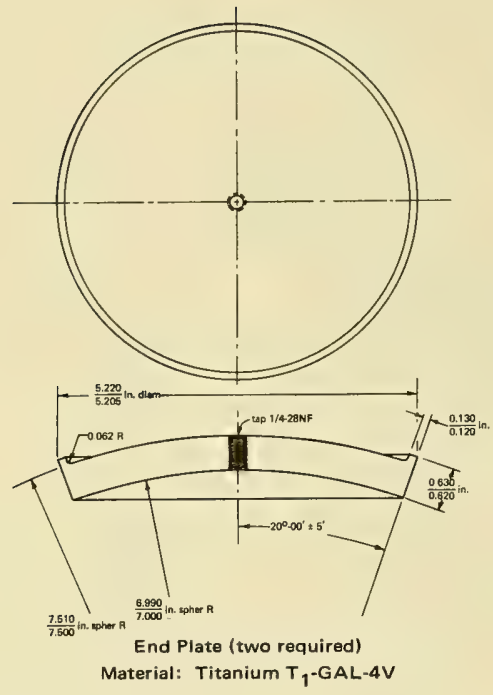

Figure 65. Heavy-duty polar penetration closures for low temperature implosion testing of 15-inchdiameter model capsules.
Hydrostatic loading of the capsules was performed in NCEL's 18-inch-diameter pressure vessel with a 20,000-psi pressure rating. Pressure was raised at a 90 to $110-\mathrm{psi} / \mathrm{min}$ rate based on the maximum planned descent rate for the large-scale operational NEMO system. The pressure was generated with pneumatically powered positive displacement pumps. Their operation generated some pulsations during the pressurization, but the small magnitude of these pulsations (approximately $10 \mathrm{psi}$ ) was considered insignificant for the purpose of these hydrostatic tests. In all of the short-term tests, the average pressurization rate of $100 \mathrm{psi} / \mathrm{min}$ was maintained until implosion of the model occurred. After implosion, the pressure vessel was opened and the fragments inspected for clues pointing to the source of failure (Figure 69).

Prior to performance of the short-term tests to implosion, some of the models were subjected to other types of hydrostatic tests. For example, Models 8 and 15 were subjected to cyclic loading prior to their implosion under short-term test conditions (see Table 6). Although subjecting the same model to other test conditions prior to short-term implosion testing is generally not desirable, the small number of models available for the evaluation of the capsule design made this test policy necessary.

Long-term tests were performed on 13 NEMO models (Table 5). These tests were to provide data on the structural response of acrylic plastic hulls to sustained hydrostatic loadings of different magnitudes. After the models imploded under the sustained loading, the duration of loading prior to implosion at a given pressure level would be compared to the short-term implosion pressure. The difference between these pressures would constitute a valid measurement of strength degradation as a function of load duration. Several pressure levels had to be chosen for the long-term test program to establish with sufficient accuracy the relationship between implosion pressure and duration of sustained loading. 


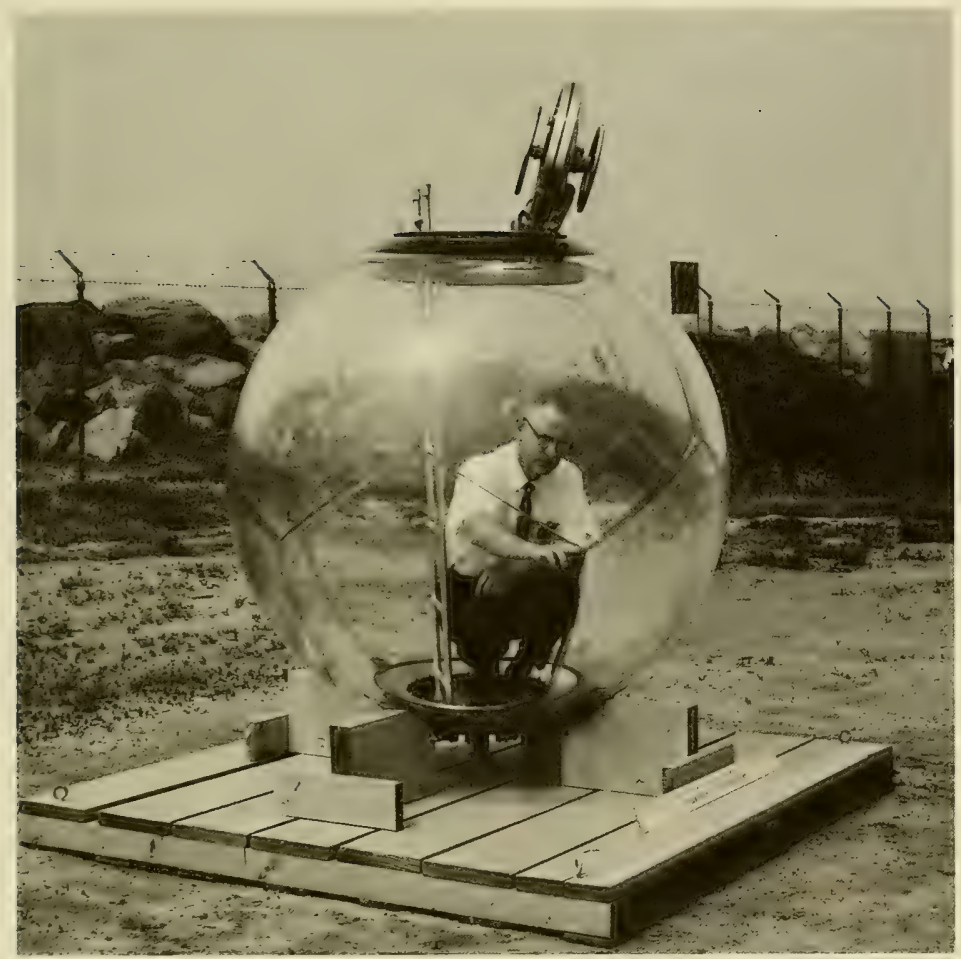

Figure 66. Large-scale capsule of 66-inch diameter for the NEMO system.

The pressure levels chosen for the long-term testing program were 1,300 psi, 1,200 psi, 1,100 psi, 1,000 psi, 900 psi, 750 psi, 500 psi, and 250 psi.* The large spread between the highest and lowest pressures selected for this testing program was based on the rationale that while the high-pressure loadings will cause the models to fail rapidly and thus permit generation of many data points within a short period of time, the lowpressure loadings will show that if the pressure is sufficiently low, implosion under long-term loading will not occur within any reasonable dive duration at operational depth. Using the data from both the high-and low-pressure tests, it will be possible to predict by interpolation the duration of loading preceding implosion for any sustained pressure level.

* Some tests were performed also at pressures of 4,500 psi, 4,000 psi, 3,500 psi, and 3,000 psi, but the model capsules used for those tests were 15-inch-OD x 13-inch-ID (Appendix E). 


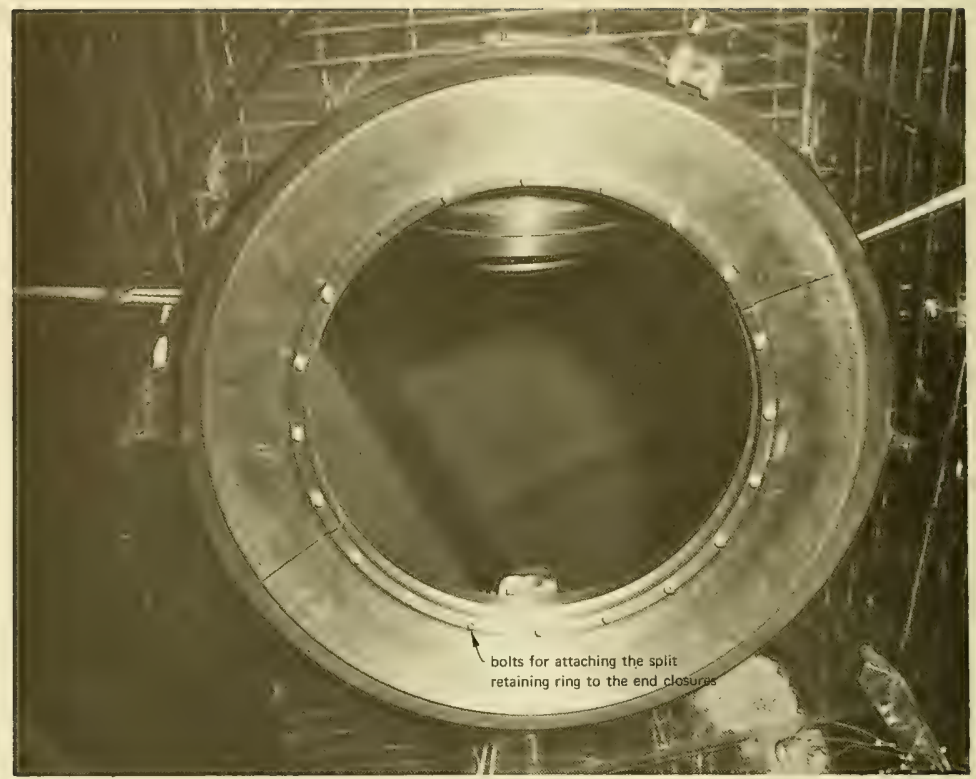

Figure 67. Split hatch retaining ring for top and bottom polar closures of the 66-inch-diameter capsule: view from the interior of the capsule.

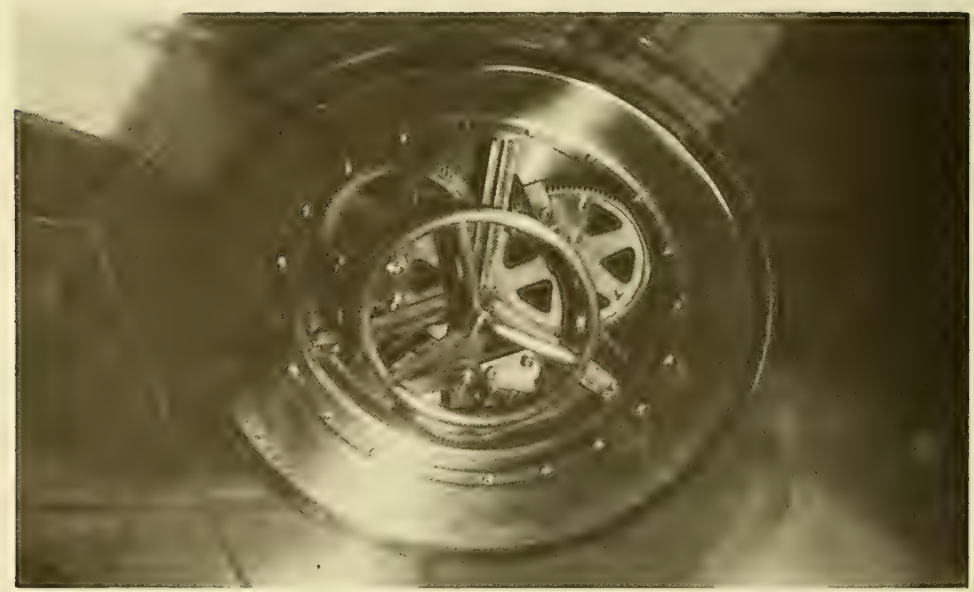

Figure 68. Hatch for the 66-inch-diameter capsule: view from the interior of the capsule. 


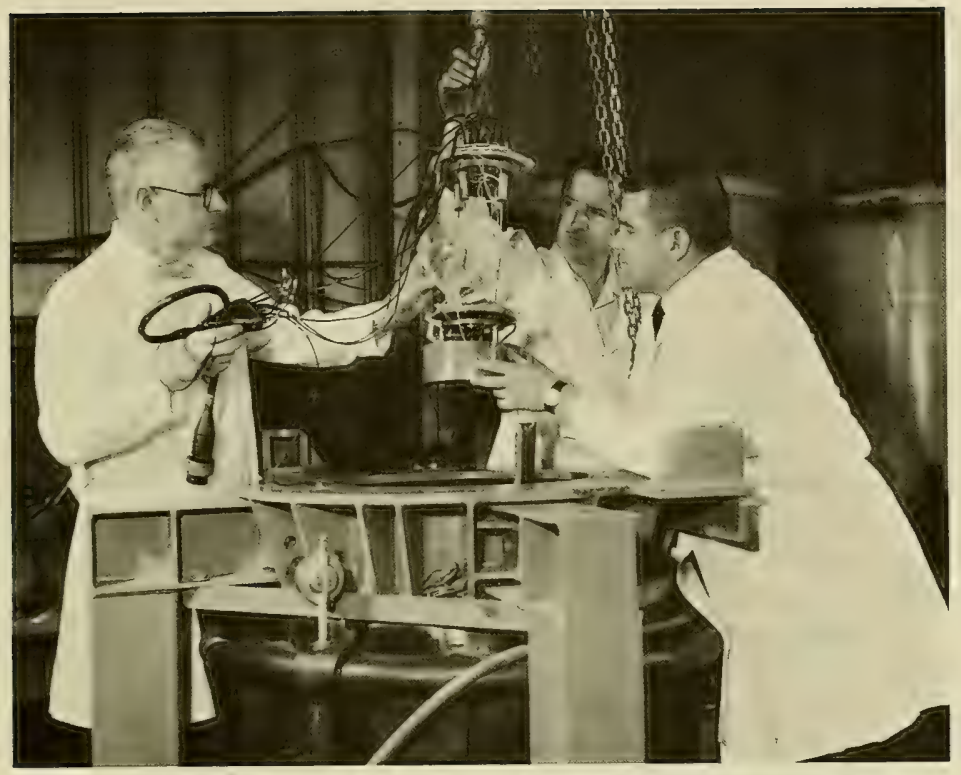

Figure 69. Removal of imploded 15-inch-diameter capsule from the 18-inch-diameter pressure vessel.

Models were tested under long-term loading conditions in hydrostatic pressure vessels specially built for this project. The pressure was raised at a 100 -psi/min rate to the desired pressure level by pumping tap water into the vessel with pneumatically powered positive displacement pump. When the desired pressure level was reached, the line to the pump was closed. Since the pressure inside the vessel varied from day to day because of variations in room temperature and the progressive contraction of the hull under hydrostatic loading, daily pressure corrections were necessary. These pressure corrections were accomplished by opening the valve in the pipe connecting the vessel to the pump and increasing the pressure, or by opening the valve in the drainline and decreasing the pressure. The day-to-day pressure variations were generally less than $10 \mathrm{psi}$, although on some occasions they were as large as 70 psi when sudden temperature changes took place in the uninsulated building where the vessels were located.

The models undergoing the long-term tests were not filled with water prior to placement in the vessel, but were simply vented to the ambient atmosphere. Because of the large buoyancy that the models possess, lead ballast was attached to the bottom plates in the models. This test condition better simulated the loading conditions to which a large-scale capsule will be 
subjected during its life as a part of the NEMO system. Some of the models (Table 5) were equipped with operational end plates while others had the simulated polar plates. Model 1 with operational polar plates was without tie rods. All models with simulated polar plates were pretensioned by means of the rods. The operational end plates were connected by means of four tie rods (Figure 57), and the simulated polar plates were connected by a single tie rod (Figure 64).

Two of the model capsules (2 and 11) were subjected to cyclic pressurization (Table 6 ) before being tested to destruction in the long-term tests. Because of their previous test history, a somewhat lower performance level during the long-term tests could be expected, and if it actually occurred, could be accounted for.

Cyclic tests were performed on four models (Table 6). The reason for choosing such a low number of models for cyclic tests was not a lack of appreciation for these tests but a lack of appropriate controls on the pumping system available for performance of cyclic pressurization tests. Different durations of cycles were selected for testing each model (Table 6). This decision was based on the rationale that since the effect of cycle duration on the cyclic life of an acrylic plastic pressure hull is unknown, cyclic tests with different durations must be utilized to cover the range of cycle profiles to which the NEMO system may be exposed during actual operation.

Pressurization and depressurization were both accomplished at a $100-\mathrm{psi} / \mathrm{min}$ rate by pneumatically powered positive displacement pumps. The temperature of the water during the performance of the cyclic tests was that of the ambient atmosphere, and therefore, as in the long-term tests, the temperature of the water varied from day to day within the 50 to $80^{\circ} \mathrm{F}$ range. The variations in ambient temperature were accompanied by variations in pressure as the water in the pressure vessel expanded or contracted with temperature. The variation in maximum pressure was particularly pronounced for the cyclic tests with long dwell time at the maximum pressure. The pressure, however, never exceeded 550 psi or went below 400 psi during dwell time at the nominal maximum pressure of 500 psi.

All the capsule models subjected to cyclic loading had no previous pressurization history except for Model 2, which had previously undergone two cyclic tests to 750- and 1,000-psi pressure levels with varying dwell times at maximum and minimum pressure. Since no damage in the model was observed prior to placing it in the regular cycling program, the effects of prior cycling history on the test results generated in the regular cycling program were considered insignificant. All of the models used for cycling tests were equipped with tie rods. 
An axial compression test was performed on a single model capsule by pretensioning the internal tie rods connecting the polar metallic end plates. The objective of this test was to establish experimentally how serious the stresses would be in the acrylic plastic hull if the pretensioned tie rods were utilized in the capsule to place it under precompression prior to lifting it off the deck during launching operations. To simulate actual lifting operations, the tie rods inside the model were pretensioned to exert a total force of 100 pounds-equal in magnitude to the weight of the pod and anchor required for a 15-inch NEMO system. Since the weight of the pod and anchor increases with the cube of the diameter, while the wall section increases as the square of the diameter, the strains from testing the model would have to be scaled up for prediction of strains in larger capsules.

Testing the Large-Scale Capsule. Although the 66-inch large-scale capsule was also tested hydrostatically, there was a distinct difference between testing it and the model capsules described previously. While for the testing of models an attempt was made to provide a separate model for each kind of test, only one large-scale capsule specimen was available for the whole series of tests planned for the large-scale prototype. For this reason, the test results generated with the single large-scale capsule must be considered from the purely experimental viewpoint to a large measure as qualitative rather than quantitative. From the operational viewpoint, the fact that a single hull has been subjected to a diversity of tests is of course desirable, rather than objectionable because, during the operational life of a functional NEMO system, the capsule will also be subjected to a diversity of hydrostatic loading conditions.

All the hydrostatic tests on the large-scale capsule were performed in NCEL's 72-inch-diameter pressure vessel (Figure 70) which has a 5,500-psi pressure rating. Since the prime objective of the testing program for the single large-scale capsule was to generate a maximum of data in the shortest period of time without imploding the hull, only tests of short duration ( 24 hours or less) were performed, as they satisfied both requirements of the test program.

The pressurization rate for all the tests (Table 7) was selected to be $100 \mathrm{psi} / \mathrm{min}$, identical to the pressurization rate applied to the capsule models. The maximum cycle pressure and temperature of the pressurizing medium varied from 100 to $1,070 \mathrm{psi}$ and 32 to $70^{\circ} \mathrm{F}$, respectively. For most of the tests, the interior of the hull was dry and open to atmospheric pressure; for a few of the cycling tests the interior was filled with water, which was vented to the atmosphere. 


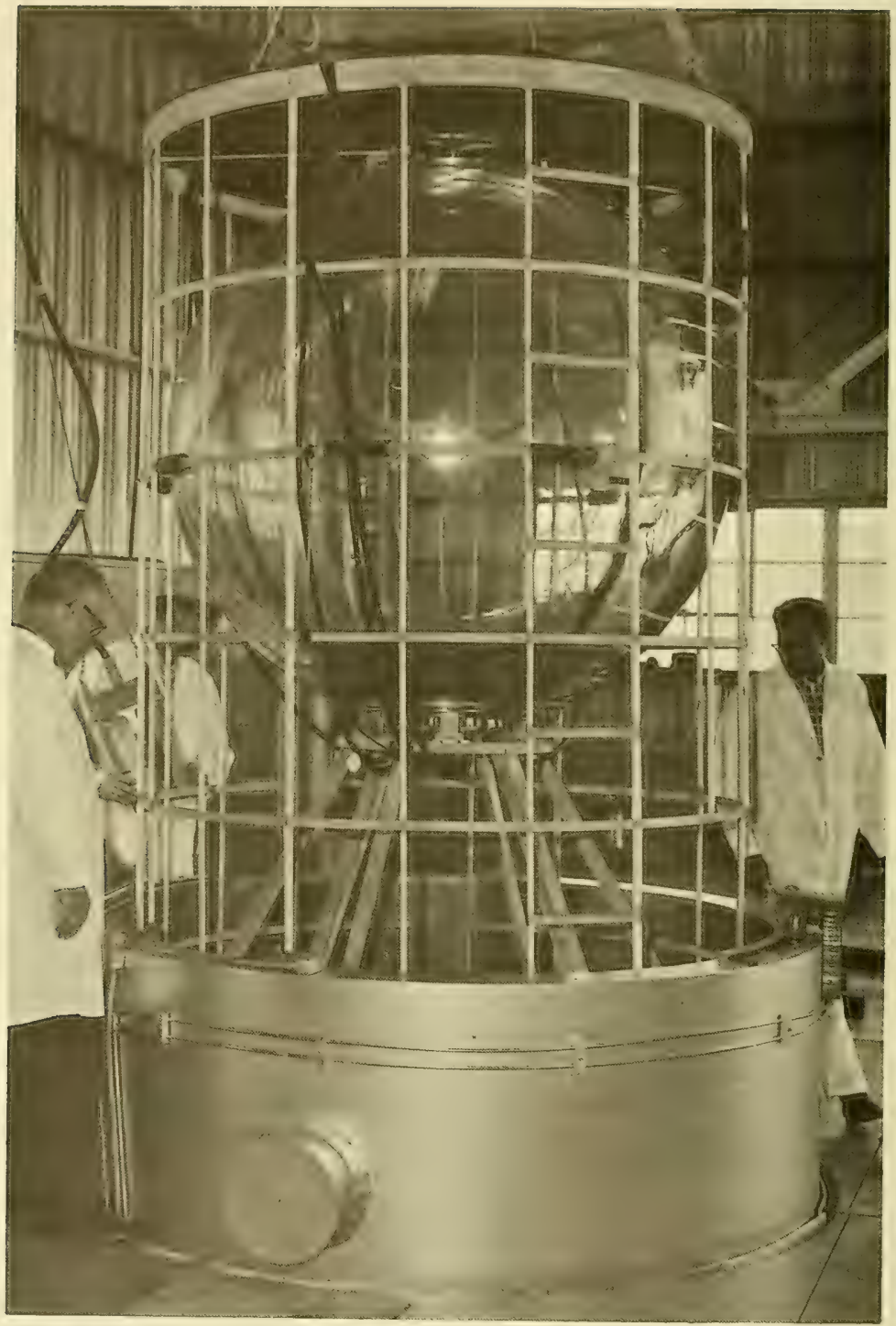

Figure 70. Placement of 66-inch-diameter capsule in the 72-inch-diameter pressure vessel at NCEL's Deep Ocean Laboratory. Note the cagelike test jig. 
Table 7. Pressurization Tests on the Prototype 66-Inch-OD $\times 61-$ Inch-ID Acrylic Plastic Capsule

\begin{tabular}{|c|c|c|c|c|c|c|}
\hline $\begin{array}{c}\text { Type } \\
\text { of } \\
\text { Test }\end{array}$ & $\begin{array}{c}\text { Number } \\
\text { of } \\
\text { Tests }\end{array}$ & $\begin{array}{l}\text { Maximum } \\
\text { Pressure } \\
\text { (psi) }\end{array}$ & $\begin{array}{c}\text { Duration of } \\
\text { Sustained } \\
\text { Loading }\end{array}$ & $\begin{array}{c}\text { Temperature } \\
\left({ }^{\circ} \mathrm{F}\right)\end{array}$ & Instrumentation & Damage \\
\hline \multirow{16}{*}{ Long term } & 1 & 100 & $24 \mathrm{hr}$ & $68-70$ & strain gages & none \\
\hline & 1 & 200 & $24 \mathrm{hr}$ & $68-70$ & strain gages & none \\
\hline & 1 & 300 & $24 \mathrm{hr}$ & $68-70$ & strain gages & none \\
\hline & 1 & 400 & $24 \mathrm{hr}$ & $68-70$ & strain gages & none \\
\hline & 1 & 500 & $24 \mathrm{hr}$ & $68-70$ & strain gages & none \\
\hline & 1 & 600 & $24 \mathrm{hr}$ & $68-70$ & strain gages & none \\
\hline & 1 & 700 & $24 \mathrm{hr}$ & $68-70$ & strain gages & none \\
\hline & 1 & 800 & $24 \mathrm{hr}$ & $68-70$ & strain gages & none \\
\hline & 1 & 250 & $6 \mathrm{hr}$ & $68-70$ & water displacement & none \\
\hline & 1 & 500 & $6 \mathrm{hr}$ & $68-70$ & water displacement & none \\
\hline & 1 & 750 & $6 \mathrm{hr}$ & $68-70$ & water displacement & none \\
\hline & 1 & 500 & $4 \mathrm{hr}$ & 38 & strain gages & none \\
\hline & 1 & 600 & $7 \mathrm{hr}$ & 36 & strain gages & none \\
\hline & 1 & 700 & $5-1 / 2 \mathrm{hr}$ & 43 & strain gages & none \\
\hline & 1 & 750 & $8 \mathrm{hr}$ & 42 & strain gages & none \\
\hline & 1 & 850 & $8 \mathrm{hr}$ & 41 & strain gages & none \\
\hline \multirow{9}{*}{ Cyclic } & 5 & 500 & $6 \mathrm{hr}$ each test & $68-70$ & strain gages & none \\
\hline & 5 & 500 & $1 \mathrm{hr}$ each test & $68-70$ & strain gages & none \\
\hline & 7 & 500 & 15 min each test & $68-70$ & strain gages & none \\
\hline & 13 & 500 & 2 min each test & $68-70$ & strain gages & none \\
\hline & 20 & 500 & $4 \mathrm{hr}$ each test & $32-75$ & strain gages & none \\
\hline & 15 & 550 & $1 \mathrm{hr}$ each test & $32-40$ & none & none \\
\hline & 13 & 550 & $1 / 2 \mathrm{hr}$ each test & $32-40$ & none & none \\
\hline & 11 & 500 & $1 \mathrm{~min}$ each test & $32-40$ & none & none \\
\hline & 1 & 1,070 & $10 \mathrm{~min}$ & 34 & none & none \\
\hline Short term & 1 & 1,850 & none & 70 & none & imploded \\
\hline
\end{tabular}

Notes: 1. For all the tests the 66 -inch capsule was secured to the protective cage by bolting the polar steel plate on the bottom of the capsule to the base of the cage.

2. The capsule was tested a total of 107 times.

3. The capsule was removed 25 times from the pressure vessel for inspection during the test program.

4. The capsule sustained a total of 375 hours under hydrostatic pressure prior to destructive implosion test. 
For the performance of all the hydrostatic pressure tests, a special cagelike holding jig was designed and fabricated for NEMO. Its purpose was to protect the hull against impact when the capsule was placed into the vessel and to act both as an anchor and as a support for the either positively or negatively buoyant hull (depending on whether its interior was dry or filled with water). When the interior of the hull was dry, it exerted approximately 4,000 pounds of positive buoyancy force on the bottom plate anchored to the holding jig.

Although all the tests planned for the large-scale capsule were basically of cyclic nature, the objectives of these tests varied from one series of tests to another. In order to clarify these objectives, each series of tests will be discussed below in detail.

Time dependent strain under sustained pressure loading was to be determined by pressurizing the hull to the desired pressure level and holding it at that pressure until the time-dependent rate of strain increase stabilized itself for that pressure. Since the time-dependent strain rate varies from one sustained pressure level to another, the hull had to be held at different sustained pressures for a sufficient time to establish the strain rates. The test procedure established for this series of tests consisted of pressurizing the hull to 100-, 200-, 300-, 400-, 500-, 600-, 700-, and 800-psi pressure levels and holding it at each one of those levels for at least 24 hours. At the conclusion of every sustained pressure test, the pressure was decreased to $0 \mathrm{psi}$, so that the hull could relax between pressurization cycles. The relaxation periods varied from 45 to 188 hours. For this series of tests, the interior of the hull was dry and vented to the atmosphere. The ambient temperatures of the hull interior and exterior were maintained in the 68 to $72^{\circ} \mathrm{F}$ temperature range.

The effect of cycling on the magnitude of strains in the hull was to be investigated by pressurizing the hull to 500 psi, holding it at that pressure for a period of time, depressurizing it to $0 \mathrm{psi}$ and holding it at that pressure for the same length of time as at maximum pressure. Since the relaxation period at 0 psi was not necessarily always sufficient to permit complete relaxation of the hull, some residual compressive strain would remain in the hull at the beginning of the new pressure cycle and would influence the strain during the succeeding pressurization cycle. By varying the length of dwell time at the 500-psi level in each cycle, the magnitude of residual strain in the hull was expected to vary from one series of cycles to another. By comparing the strain rates in the hull generated during long and short cycles, some indication of the effect of residual strains on the strain rates could be obtained. To vary the dwell time as much as possible, the dwell time at 500 psi was varied from 360 minutes to 2 minutes. For this series 
of tests, the interior of the hull was kept dry and vented to the atmosphere. Ambient temperatures of the hull interior and exterior were maintained in the 68 to $72^{\circ} \mathrm{F}$ temperature range.

Displacement changes of the hull were investigated by a series of tests in which the pressure was varied from 250 to 750 psi. In each of the tests, the pressure was raised to the desired maximum pressure level, held there for 6 hours, and then followed by a depressurization and relaxation period of 18 hours. For those tests, the interior of the hull was filled with water vented to the atmosphere. During the pressurization of the hull, water was displaced from it because of the hull contraction, and the quantity of displaced water was used as a measure of hull contraction. The ambient temperatures both in the exterior and interior of the hull were in the 68 to $72^{\circ} \mathrm{F}$ range. Upon depressurization, the water displaced from the interior was siphoned back into the vessel interior, giving a measure of capsule relaxation during depressurization.

The effect of differential temperature on the strains in the hull was evaluated in a series of cyclic pressure tests from 0 to 500 psi with the dwell time at 500 psi being 4 hours and the relaxation time 20 hours. The temperature of the water surrounding the capsule was varied from 60 to $32^{\circ} \mathrm{F}$, while the ambient temperature of the dry interior varied from 79 to $45^{\circ} \mathrm{F}$. Because of the difference in internal and external ambient temperatures, a temperature gradient existed inside the wall of the acrylic plastic hull (Figure 71 ).

In addition to the tests to 500 psi, one brief test was run to 1,070 psi and another to 1,000 psi. Besides showing the capability of the hull to withstand a pressure $300 \%$ greater than the rated capability of the hull, these tests also supplied information on the strains in the hull at very high stress levels and the rate of relaxation after depressurization from such high excess pressures. The scale effect for crack-sensitive materials was determined by imploding the 66-inch capsule and comparing its short-term implosion pressure with the short-term implosion pressures previously determined by imploding 15-inch small-scale models. This test completed the testing program for the 66-inch prototype capsule.

\section{Instrumentation}

Long-term and cyclic tests on the 15-inch capsule models used only a minimum of instrumentation as it was not feasible to tie up electronic equipment for more than 1 year just for these tests. The instrumentation for almost all model capsules under long-term or cyclic pressure testing consisted only of temperature and pressure gages, from which data were read and recorded 3 times daily. The failure of the models was noted by a sudden drop in pressure. If failure did not occur prior to the termination of the test, the model was then carefully inspected upon removal from the vessel to ascertain the level of damage to the hull. 


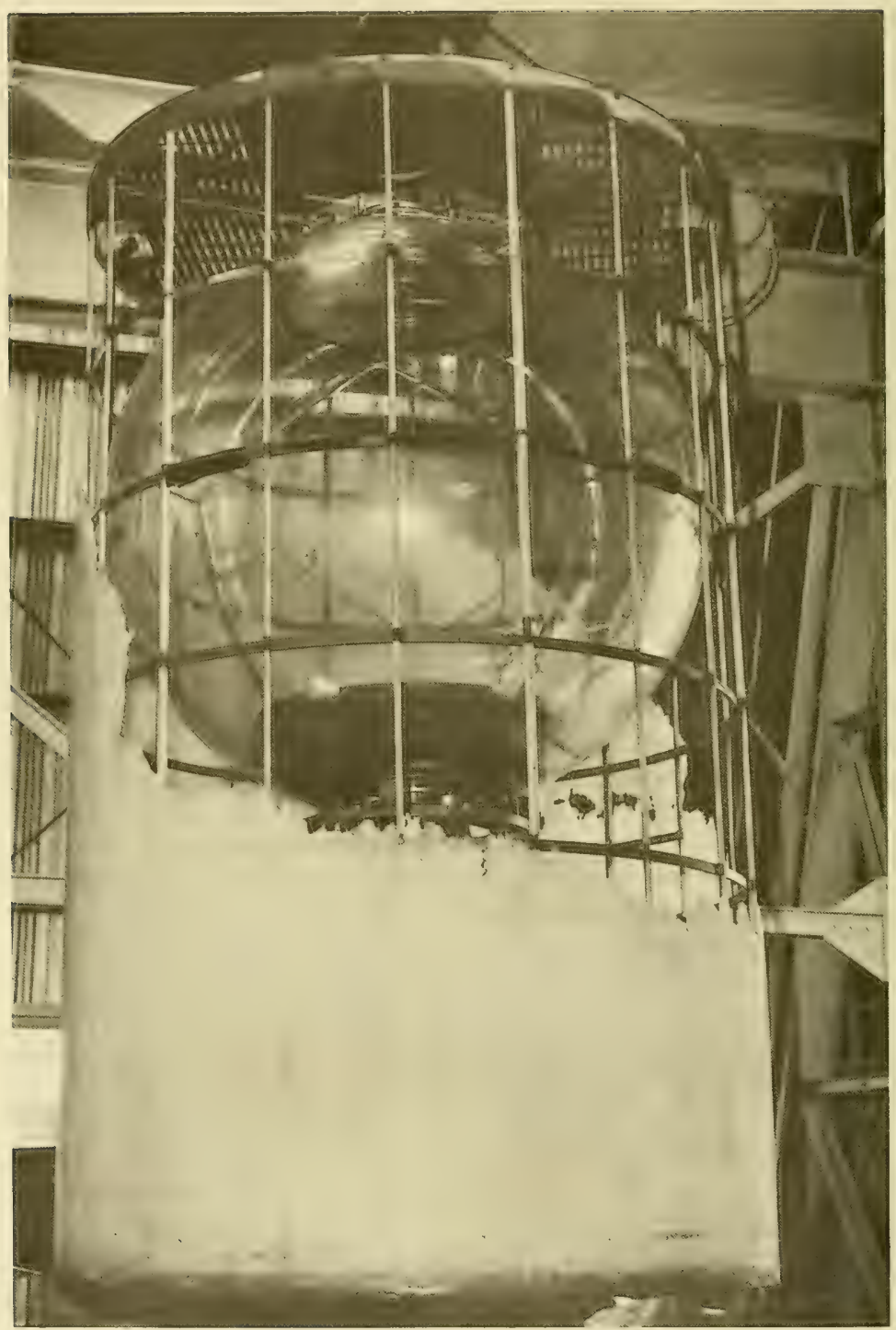

Figure 71. The 66-inch-diameter capsule after the simulated arctic dive to the 2,400 -foot depth. Note the accumulation of ice due to subfreezing water temperature. 
A few of the models were equipped with a volume change measuring system to determine the magnitude of hull shrinkage under long-term loading (Figure 72). This instrumentation system consisted of distilled water filling the total interior volume of the model and tubing connecting the interior of the model to an accurate graduate located on the exterior of the pressure vessel. The shrinkage of the acrylic plastic hull under sustained pressure caused water to flow from its interior into the graduate. Readings of water level, temperature, and pressure were, at the initiation of the test, taken at 1-minute intervals for the first 15 minutes, every 5 minutes for the next 45 minutes, hourly for the next 7 hours, and 3 times daily thereafter until the model failed or was depressurized. When the model capsule was depressurized, the same data-recording process was repeated.

Only two models were strain-gaged (Figure 73), and then only four strain gages were used because of the limitation on the number of available electric penetrations in the test pressure vessel. However, it was hoped that by judicious placement of the strain gages a good representation of strain distribution in the hull could be obtained.

Short-term tests on model capsules utilized either strain-gage (Figure 74), volume-displacement, diameter-contraction, or photoelastic instrumentation. The strain-gage and the water-displacement instrumentation systems were identical to those used in the long-term tests. The diametercontraction instrumentation consisted of a linear potentiometer inside the capsule placed between the polar steel end plates and an externally located read-out system calibrated to read in thousandths of an inch. The photoelastic instrumentation consisted of a light source inside the acrylic plastic hull, circular polarizing sheets, a window in the pressure vessel end closure, and a camera mounted on the exterior of the pressure vessel end closure (Figure 75). During some of the tests, the camera was removed and visual observations of the photoelastic interference fringes were made instead.

Considerably more effort and expense were devoted to the instrumentation of the large-scale capsule because (1) only a single capsule specimen existed and (2) the testing of the large capsule was not as lengthy as that of models so that complex electronic equipment could be tied up for the tests. Some of the instrumentation systems were identical to those used on the models, while others, such as the strain-instrumentation system, were considerably more complex than a similar system on the model. The instrumentation for measurement of strains consisted of 81 channels for transmitting data generated by an equal number of electric resistance strain gages mounted on the exterior and interior hull surfaces. Eastman 910 contact adhesive was found to work best for bonding the gages to the hull and DOW Corning 105 silicone rubber with associated primer for subsequent waterproofing. The strains were recorded digitally on paper by a 90 -channel BUDD DATRAN unit with a response capability of approximately 1 channel per second. Considerable trouble was encountered in the procurement of 
waterproof instrumentation cables (Figures 76 and 77) that were not overly sensitive to hydrostatic pressure. Oversensitivity would unduly influence the strain readings recorded by the externally located balancing and recording unit. Even though care was taken in selection of cables, the recorded strain data may include some of the effect of hydrostatic pressure on the instrumentation cables. Tests conducted on the instrumentation cables have shown that although the effect of pressure on the cables was generally less than $10 \times 10^{-6} \mathrm{in}$./in. (10 $\mu \mathrm{in}$./in.) on the strain recording, a few of the cables were overly sensitive and increased the strain reading by as much as $100 \mu \mathrm{in} . / \mathrm{in}$.

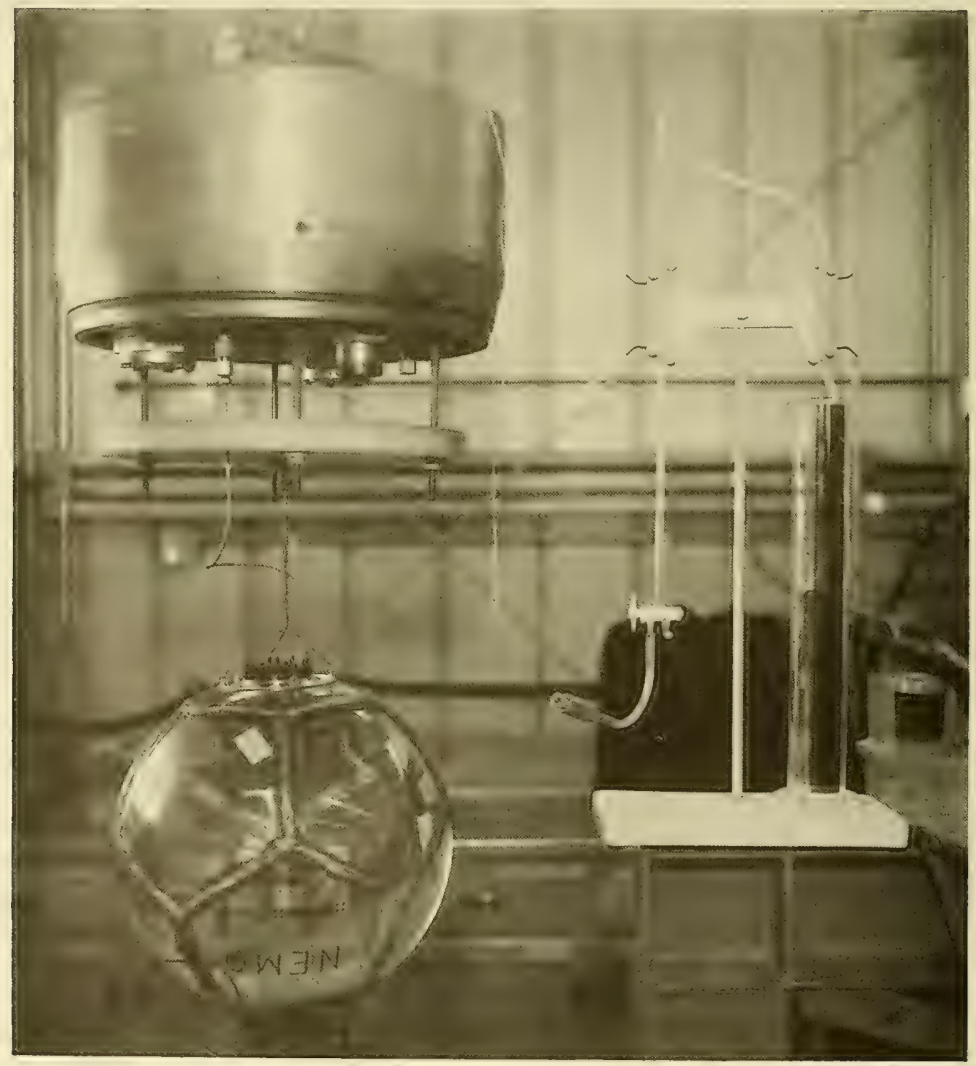

Figure 72. Typical test setup for measuring the change in volume of 15-inch-diameter model. 


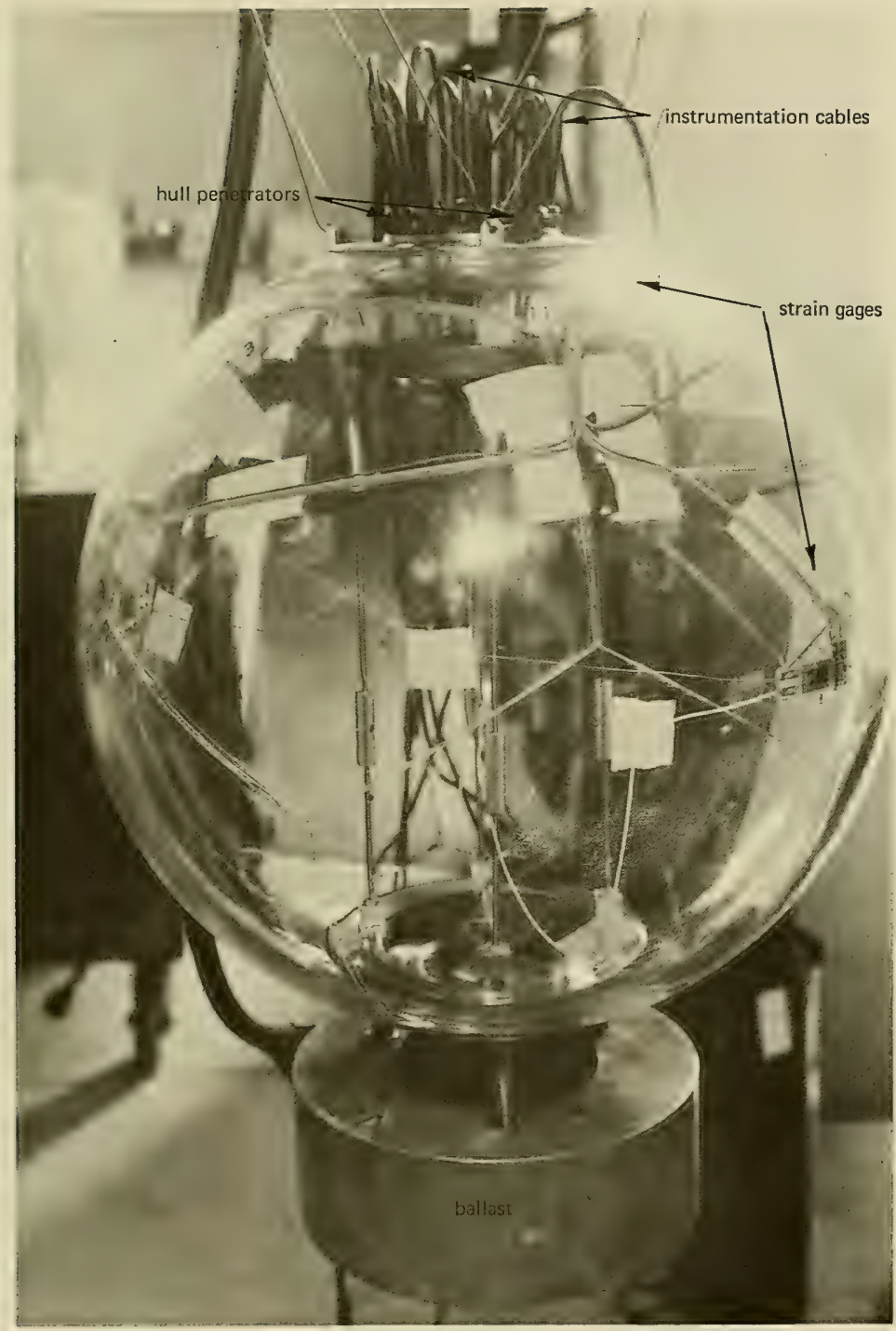

Figure 73. Typical strain gage instrumentation on a 15-inch-diameter capsule model: overall view. 

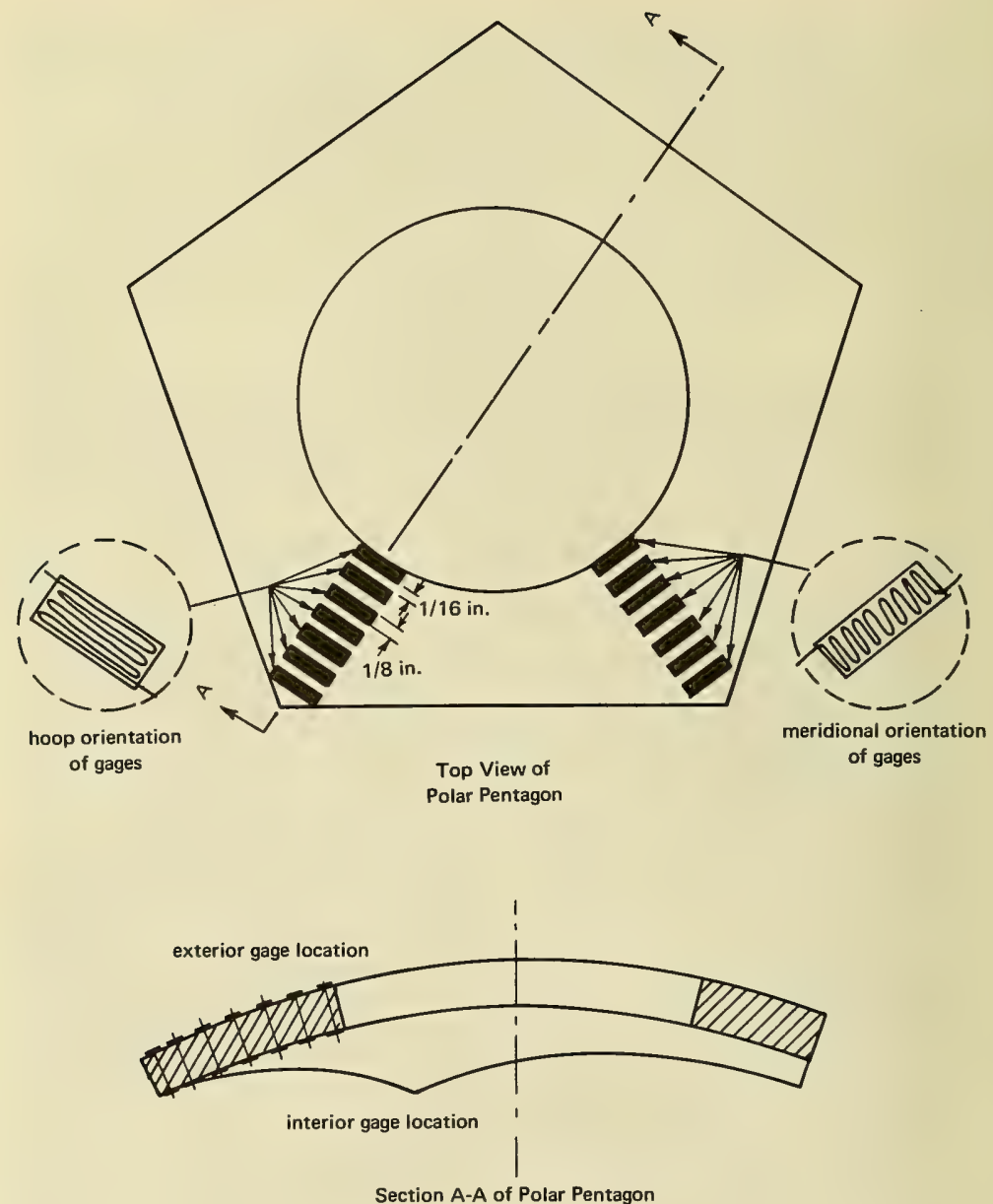

Figure 74. Location of strain gages on the 15-inch-diameter capsule near the polar openings. 


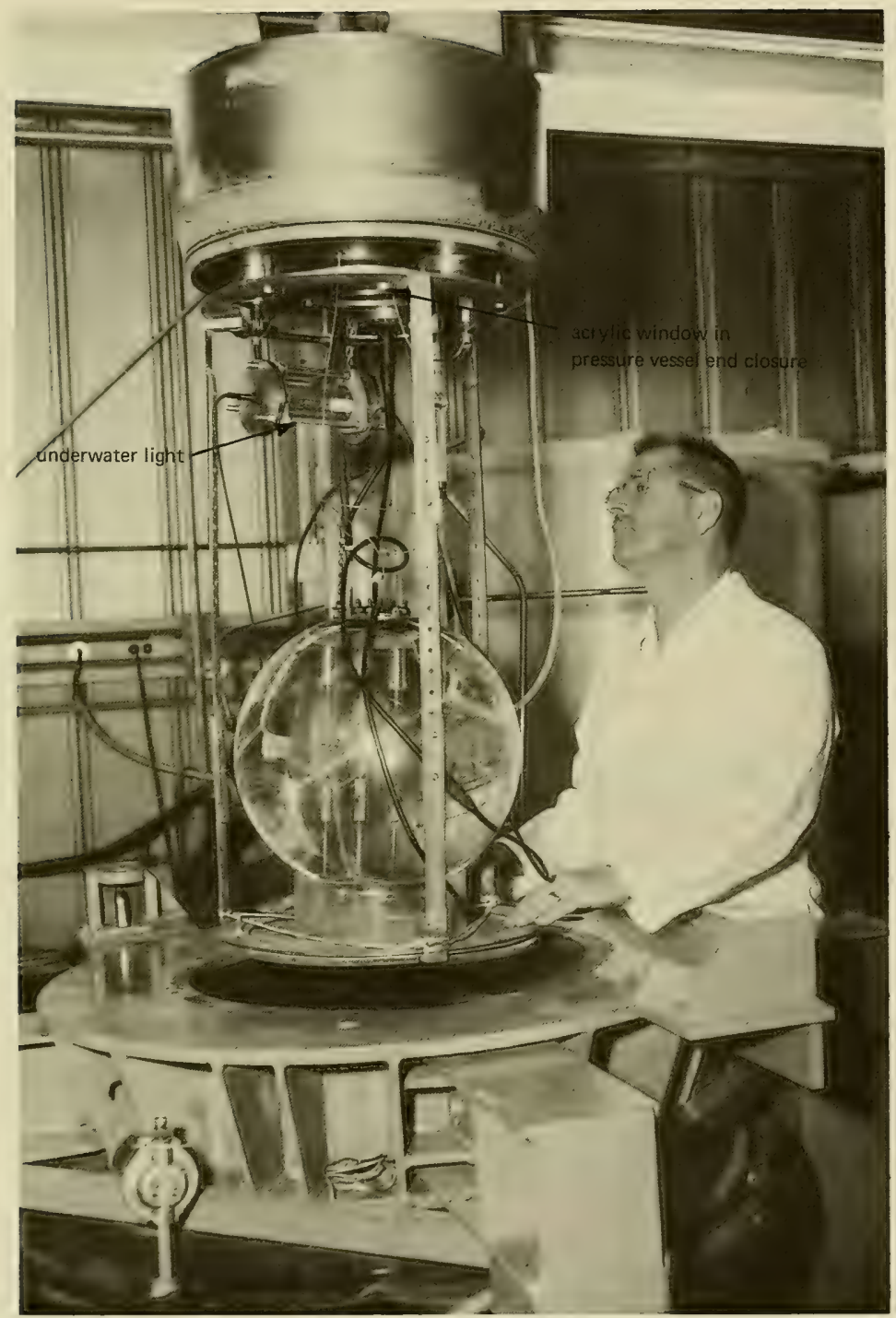

Figure 75. Test setup for measuring photoelastically the strain concentration around the steel polar insert in the 15-inch-diameter capsule under external hydrostatic pressure. 


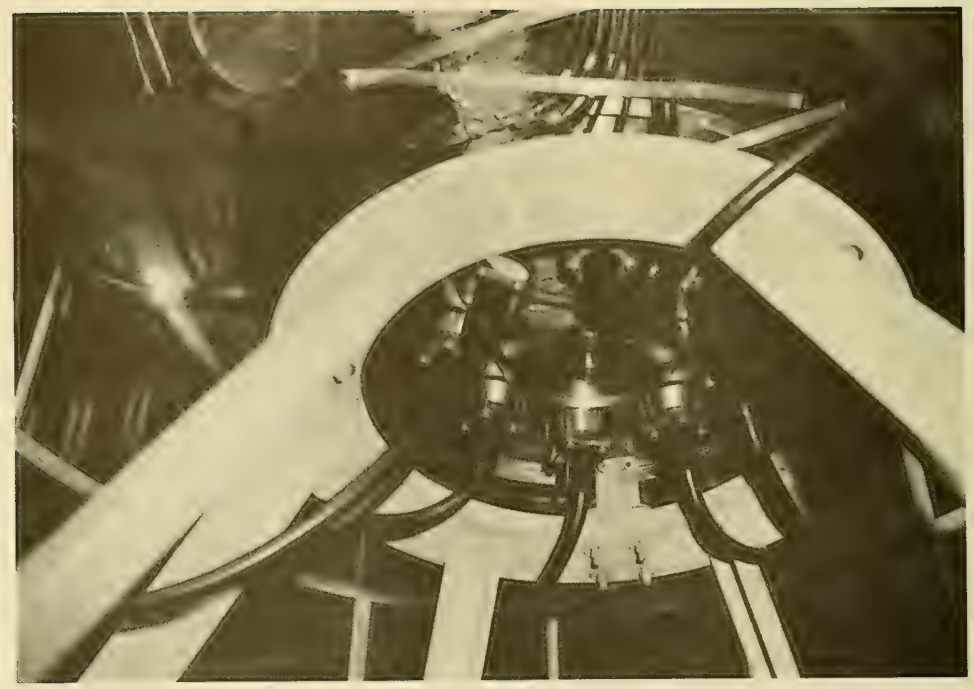

Figure 76. Cables and cable feedthroughs in the steel polar penetration closure for the strain gage instrumentation on the 66-inch-diameter capsule.

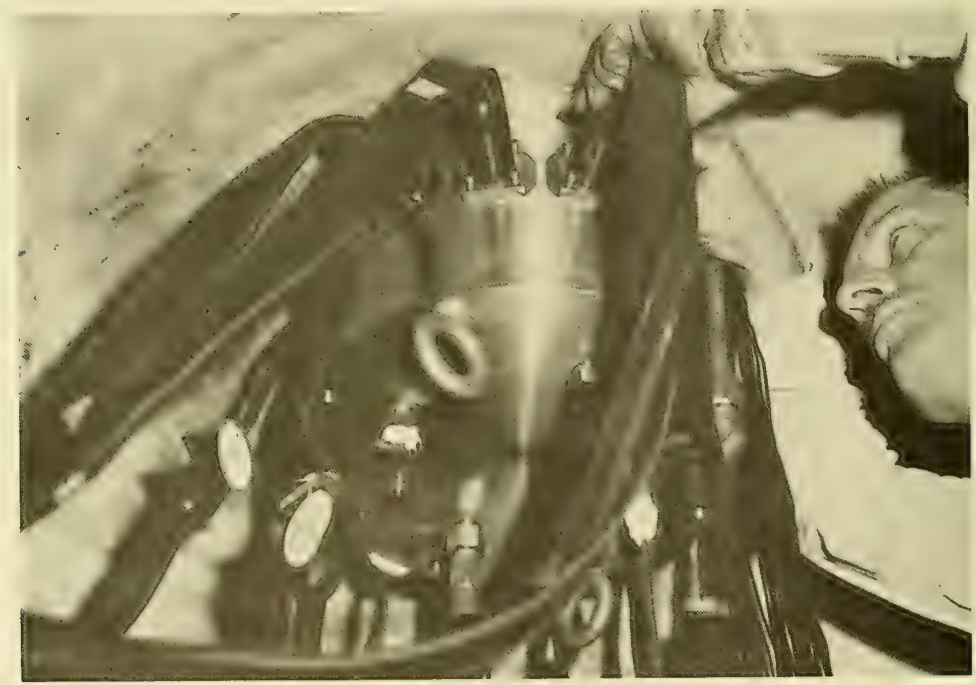

Figure 77. Termination of instrumentation cables at the head of the 72-inch pressure vessel. 


\section{TEST FINDINGS}

\section{Model Capsules}

Short-term tests performed on 11 model capsules generated data on which several important findings are based:

1. The short-term implosion pressure of model capsules of same dimensions, construction, and dimensional tolerances tested at constant ambient temperature is repeatable from one sphere to another with only small deviations from the average (Table 4). For example, four NEMO capsules tested in the 59 to $68^{\circ} \mathrm{F}$ temperature range failed in the 1,520 to-1,645-psi pressure range, indicating less than a $\pm 5 \%$ deviation from the group's average of 1,578 psi.

2. The presence of metal end plates in the acrylic plastic sphere does not significantly alter its short-term implosion pressure, as can be seen by comparing the 1,360-psi implosion pressure of a capsule at $70^{\circ} \mathrm{F}$ without metal inserts (Model 0) with the 1,578-psi average implosion pressure at $64^{\circ} \mathrm{F}$ of the NEMO capsules with metal end plates (Table 4).

3. Ambient temperature has a significant effect on the short-term implosion pressure (Figure 78): the short term implosion pressure was found to be an inverse linear function of the ambient temperature in the 32 to $114^{\circ} \mathrm{F}$ range used for testing of models.

4. A severe temperature differential across the thickness of the hull has no significant effect on the short-term implosion pressure. The implosion pressure of an acrylic plastic sphere under temperature differential test conditions appears to be related to the average temperature of the acrylic plastic hull. The acrylic plastic sphere with $104^{\circ} \mathrm{F}$ interior and $32^{\circ} \mathrm{F}$ exterior temperatures (average temperature of $68^{\circ} \mathrm{F}$ ) failed at $1,660 \mathrm{psi}$ which corresponds approximately to the implosion pressure of a sphere having interior and exterior temperatures in the 60 to $68^{\circ} \mathrm{F}$ range (Figure 78).

5. Ultimate failure in all short-term tests was catastrophic and accompanied by a loud noise. The speed of the implosion was rather slow, the duration of the implosion being on the order of 1 second. The failure mechanism was of a general, rather than a local nature. The fragments were small and the fracture surfaces ran across bond lines indicating that the bonded surfaces were not the planes of weakness along which the failure took place. No cracks were observed on the acrylic plastic bearing surface in contact with the metal end plate. 


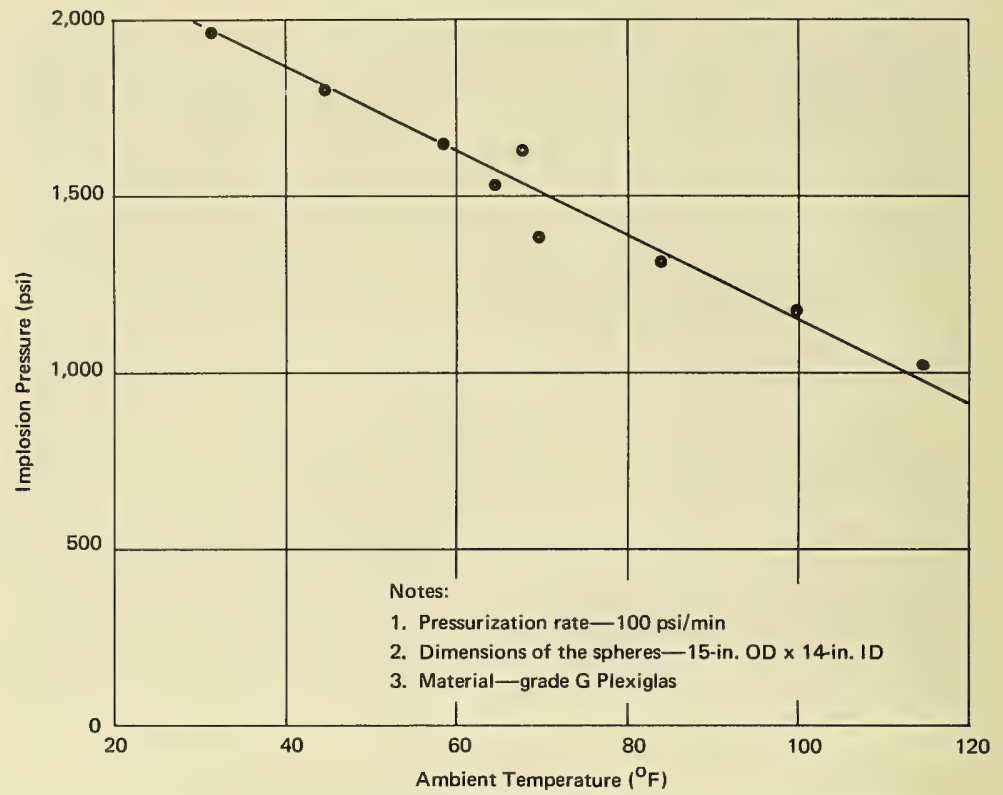

Figure 78. Implosion pressure of acrylic plastic capsule models as a function of ambient water temperature under short-term loading.

6. The magnitude of the volume decrease that the acrylic plastic spheres undergo with increase in short-term external hydrostatic pressure varies with ambient temperatures (Figure 79). The relationship between pressure and volume decrease is linear to pressures beyond 1,000 psi, except that (1) the volume decrease rate varies directly with temperature and (2) the linear-pressure-versus-volume-decrease relationship becomes nonlinear at lower pressure for high ambient temperature. Although the acrylic plastic spheres fail at different pressures, depending on the ambient temperature, the total decrease in volume at the moment of implosion is about the same for all of them: approximately $5 \%$ of the original sphere's displacement in water (equivalent to approximately 16,900 to $17,100 \mu \mathrm{in}$./in. average strain in the acrylic plastic).

7. Failure of the simple steel end plates of type 316 stainless steel used in some of the NEMO models takes place at approximately $1,800 \mathrm{psi}$ (Figure 80). The failure takes place in the form of plastic buckling. 


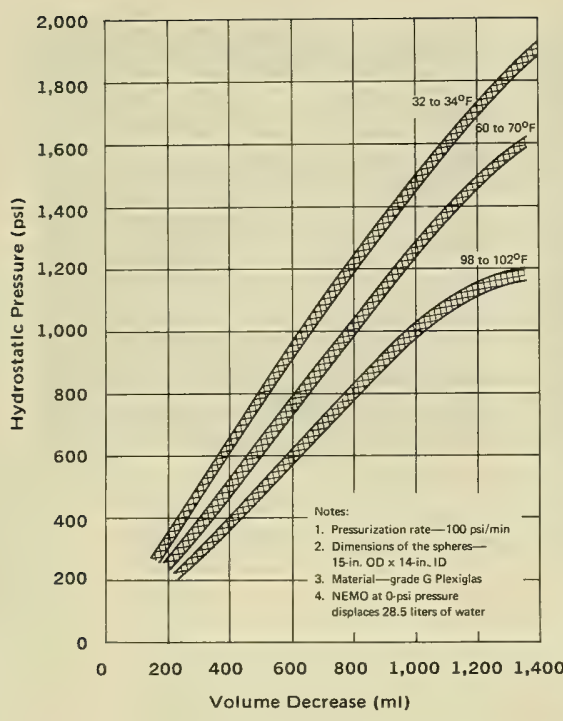

Figure 79. Change in displacement of acrylic plastic capsule models as a function of hydrostatic pressure under shortterm loading.
8. All strains measured on the interior or exterior surfaces of the acrylic plastic hull were compressive. The meridional and hoop strains on the capsule models measured at their equator were of approximately the same magnitude. Their relationship to the hydrostatic pressure under short-term loading was linear to at least a 750-psi loading (Figure 81). The strains in the immediate vicinity of the steel end plates differed from those measured at the equator. This was indicated both by the magnitudes of strains measured there with electric resistance strain gages and by photoelastic techniques. From these readings, it appears that the maximum stress encountered around the steel end plate is at the very edge of penetration on the interior surface of the sphere. The meridional stress is in the elastic range of acrylic approximately 8 to 10 times higher than the stress measured at the equator at the same pressure (Figure 82). The circumferential stresses at the same location are, on the other hand, only 2 to 3 times higher than those found at the equator (Figure 83). In general, the distribution of stresses near polar penetrations indicates that bending moments are superimposed upon the membrane stresses in the vicinity of penetration through the acrylic plastic hull. Also, since at the 500-psi pressure the stress at the equator is in the 3,000-to-4,000-psi range, plastic redistribution of stresses probably takes place in the acrylic plastic at the edge of penetration.

Long-term tests performed on 13 model capsules over a period of 3 years (Table 5) at room temperature have shown that long-term sustained pressure loading is a very important test condition for acrylic plastic pressure hulls, as this test condition causes the acrylic plastic pressure hulls to fail in a mode that is not detectable by short-term tests. The major findings resulting from the long-term sustained hydrostatic tests of NEMO models are: 

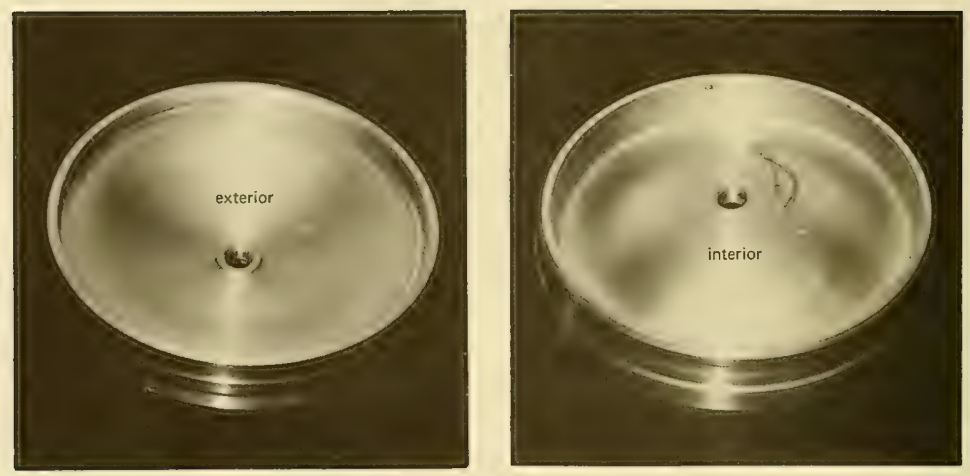

Figure 80. Simplified polar penetration closure for 15-inch-diameter capsule that failed by plastic instability and yielding at 1,800 psi of shortterm hydrostatic loading.

1. Acrylic plastic capsules under external hydrostatic pressure exhibit time-dependent strains even at hydrostatic pressures as low as 250 psi (one-half the design pressure). The time-dependent strain rate at sustained long-term hydrostatic loading is a function of hydrostatic pressure: higher pressures generate higher strain rates. The time-dependent strain rate is also an inverse function of loading duration: the strain rate is largest immediately after load application and decreases in magnitude with duration of loading (Figures 84 through 86 ).

2. The magnitude of strain in a NEMO model after a given duration of sustained loading varies linearly with the magnitude of applied loading from 0 to 800 psi. This relationship becomes (Figure 85) markedly nonlinear at hydrostatic loadings above 800 psi for room temperature ambient test conditions. Although long-term tests were not conducted at other ambient temperatures besides room temperature, it can be postulated on the basis of research conducted by others with acrylic plastic tensile test specimens that both the magnitude of strain and the magnitude of hydrostatic pressure at which the linear relationship ceases will vary with the temperature. High temperatures will cause larger strains for the same pressure and duration of loading, while low temperatures will be accompanied by low strains under long-term loading. 


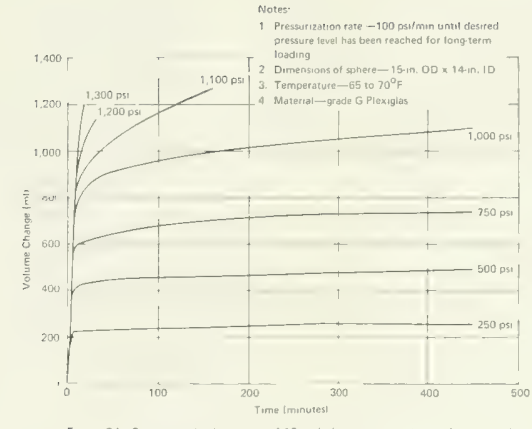

Figure 84. Cnange in displacement of 15. nnch-diameter capsule as a function of hydrostatic pressure and duration of sustaned loading, summary of
ith tests

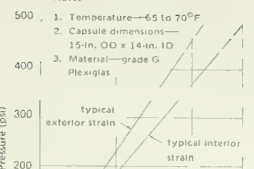

100

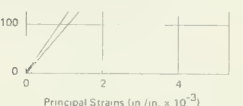

Figure 81 . Strans on the equatoni region of the 15 -inch.
diamerer caossule modes

dilameter cansule model term hydrostatic loading

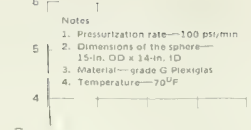

$\frac{-5}{5} 3$
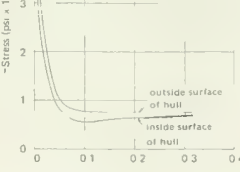

Penetration Edge fradians

Figure 82. Meridional stresses around the simplified polar pene.
tration closure as a function of distance trom the edge tere hydrostancioastiog

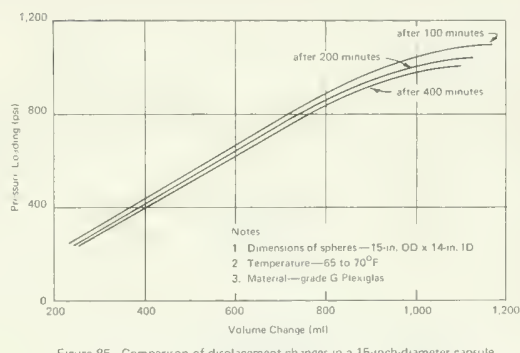

Figure 85, Comparison of displicement changes in a 15-nnch-diameter copsulia under different hydrostatic pressure toad
400 minutes of sustarned pressurization

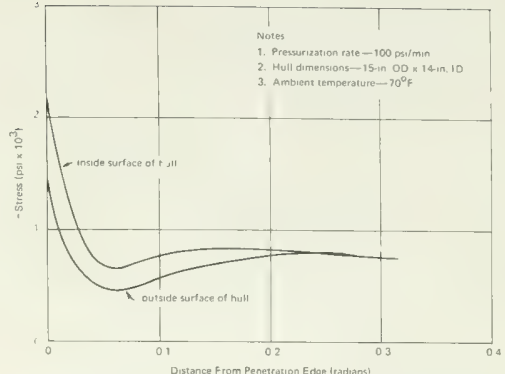

Figure 83. Hoop stresses around the simplified polar penetration closure as a function of distance
hydrostatic loading

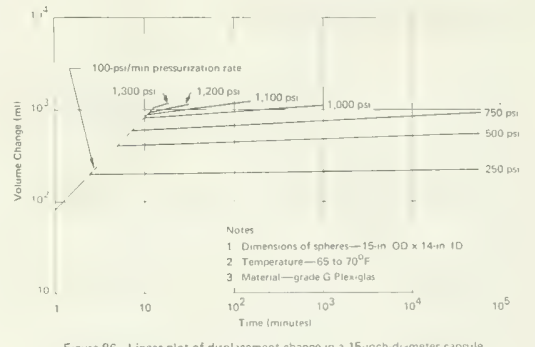

Figure 86 Linear plot of displucement change in a 15 -inch-diameter capsute under sustaned hydrostatic loading 



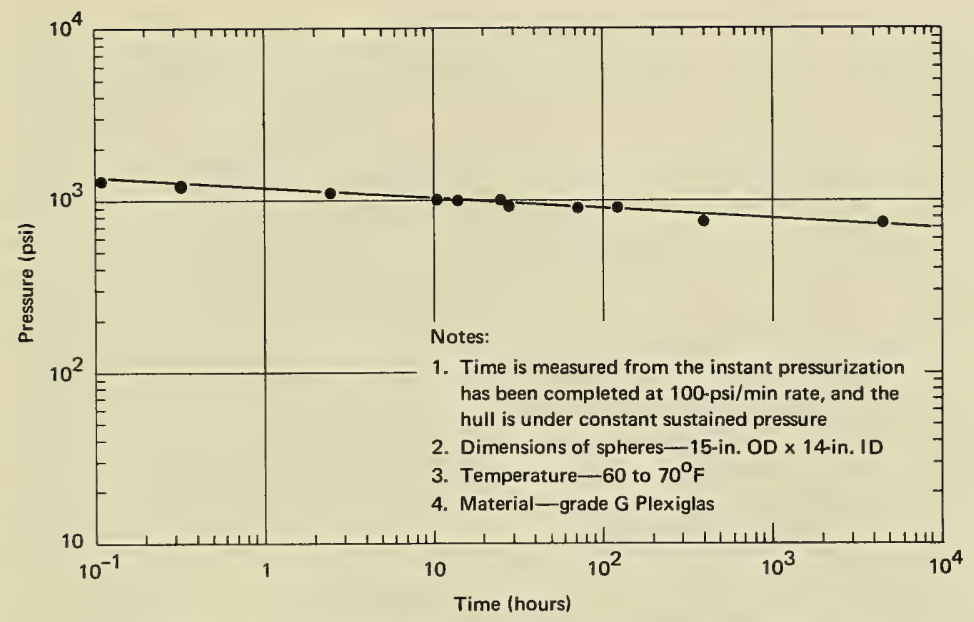

Figure 87. Implosion pressure as a function of sustained pressure duration at room temperature for 15-inch-diameter capsules.

3. Long-term sustained hydrostatic loading caused model capsules to fail at a lower pressure than short-term loading. The relationship between the magnitude of pressure and the duration of loading at which the models failed were represented by a linear graph when the test results were plotted on a log-log scale (Figure 87). When the critical pressure versus time relationship of Figure 87 was extrapolated, it was found that under a sustained pressure of $500 \mathrm{psi}$ and in the 60 to $70^{\circ} \mathrm{F}$ ambient temperature range the time-dependent implosion of the model capsule would not take place before at least 10 years of exposure. At higher temperatures, since the timedependent strain rate is higher, the implosion failure would occur sooner, while at lower temperatures, since the time-dependent strain rate is lower, the failure would occur later. For example, the model capsule under 750 psi long-term pressure loading at $70^{\circ} \mathrm{F}$ average ambient temperature failed after 366 hours, while another model ( $10 \%$ thicker) under the same magnitude of pressure loading, but at $60^{\circ} \mathrm{F}$ average temperature, failed after 4,500 hours.

4. The time-dependent failure of the model capsules was local in nature (Figure 88). It appeared to have been triggered by local deviations from sphericity and uniform wall thickness. Since local decrease in curvature or wall thickness causes a local increase in compressive membrane stresses, the time-dependent strains in this area would be higher also, thus further 
aggravating the local imperfection. Ultimately the curvature decreases locally to such an extent that the flexure stress dominates the stress field. When the tensile component of the flexure stress overcomes the compressive membrane stress at the center of the flat spot on the interior surface of the capsule, failure of the acrylic plastic occurs (Figure 89). That the local failure occurs in flexure is substantiated by (1) the similarity of the failure surface on the interior of the hull (Figure 90) to the fracture on interior of flat disc acrylic plastic windows that fail in flexure (Figure 91) and (2) by the presence of the circular crack on the exterior surface of the hull at the location where the flexure hinge encircling the flat spot was located.

5. Crazing and cracks were found on the acrylic plastic bearing surfaces in contact with the steel end plates after conclusion of long-term sustained pressure loadings at 500-, 750-, and 900-psi pressure levels (Figures 92 through 95). No cracks or crazing were found in capsules subjected to long-term sustained loading at pressures less than 500 psi or higher than 900 psi. It is hypothesized that the formation of cracks on the acrylic plastic bearing surface in contact with the end plate depends on the magnitude of timedependent strain (elastic and plastic components of strain) rather than stress in the bearing surface. The model capsule under 250-psi long-term sustained hydrostatic loading did not exhibit cracks because the magnitude of the timedependent strain at that stress level is small even after 1 year of sustained loading. The models under pressure in excess of 900 psi also exhibited no cracks because although the time-dependent strain rate at these stress levels is very high, the magnitude of time-dependent strain is low at the moment of failure, as the failure of the model occurs within a relatively short time. Only in the 500-to-900-psi range of hydrostatic loading does the magnitude of time-dependent strain in the acrylic plastic bearing surface become sufficiently large to cause cracks in the acrylic plastic surface at the steel end plate, as the stress level is fairly high and the duration of sustained loading without implosion is long.

The cracks were not uniformly distributed across the thickness of the hull at the bearing surface but were primarily concentrated on the inner half of the wall. The deepest cracks were at the location on the bearing surface that corresponded to the bottom edge of the O-ring groove in the steel end plate. The cracks were circumferential with respect to the steel end plate and propagated themselves at right angles to the bearing surface. The depth of these cracks was greater in the model that was subjected to $750 \mathrm{psi}$ for 4,500 hours (Figures 92 and 93) than in the model at 900 psi loaded for 72 hours. This could be explained by the fact that at the completion of the test the magnitude of time-dependent strain in the model loaded at $900 \mathrm{psi}$, although the stress level was higher on the latter. 


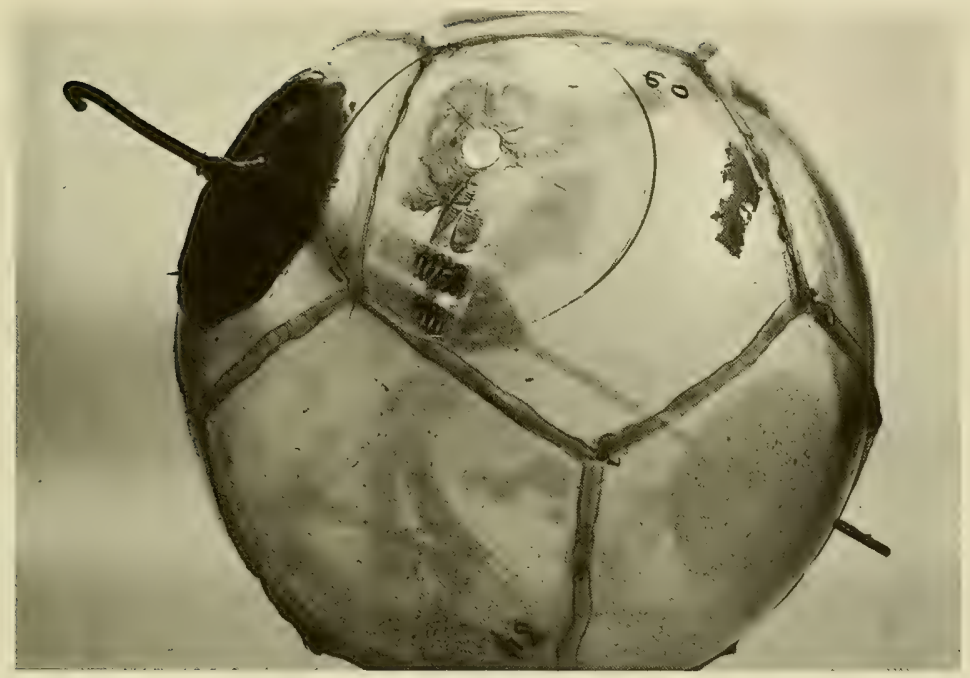

(a) Model 3.

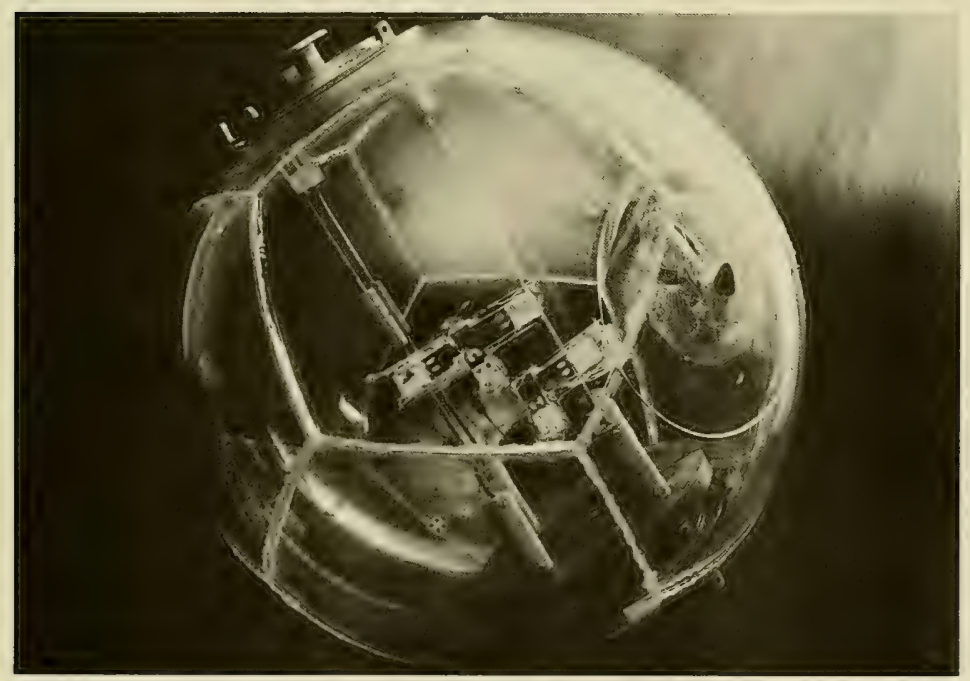

(b) Model 2.

Figure 88. Typical examples of localized, time-dependent, viscoelastic buckling of the 15-inch-diameter capsules subjected to sustained 750-psi external hydrostatic pressure in 60 -to $-70^{\circ} \mathrm{F}$ temperature range until failure took place. 
$d=$ diameter of area with a larger radius of curvature than $R_{a}$

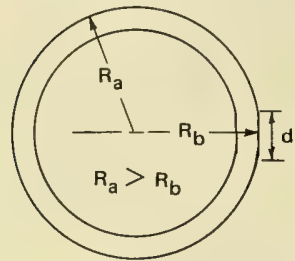

Phase 1 - Small area with larger radius resulting from imperfect fabrication.

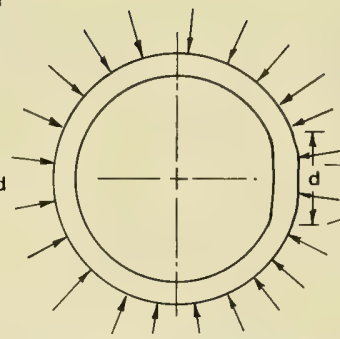

Phase 2 - Under hydrostatic loading both the area as well as the radius of curvature increase at the imperfection.

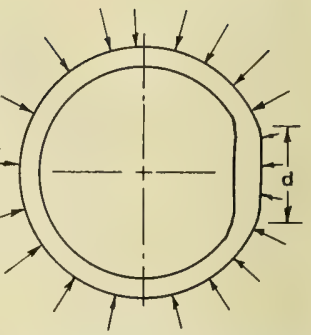

Phase 3 - The area of imperfection does not increase any more, only the radius of curvature increases.

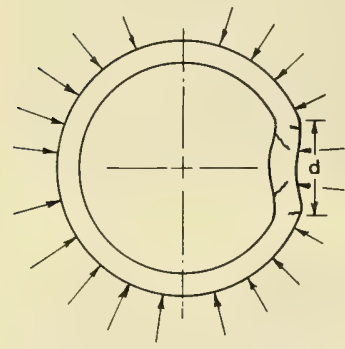

Phase 4-Area of imperfection remains constant, the direction of the radius of curvature reverses itself, fractures appear.

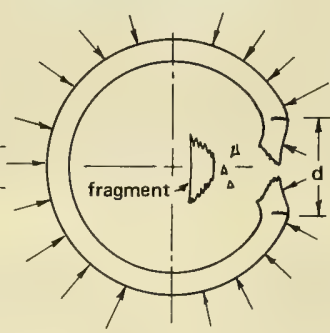

Phase 5 - Local penetration of pressure hull takes place.

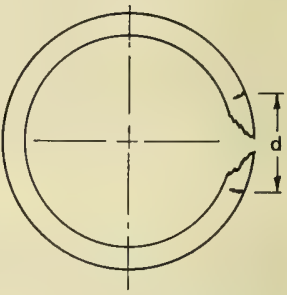

Phase 6 - Surface of the hull returns to its original sphericity at zero pressure.

Figure 89. Mechanism of localized viscoelastic failure in an acrylic plastic hull under sustained external hydrostatic pressure.

6. The total decrease in volume of model capsules at the moment of their failure under long-term sustained hydrostatic pressure was approximately the same regardless of the sustained hydrostatic pressure level at which they were kept. The total decrease of volume just prior to implosion was somewhat less than in short-term pressure tests. The magnitude of volume decrease under long-term hydrostatic pressure was approximately $4 \%$ (Figure 86 ), as compared to $4.8 \%$ under short-term pressurization (Figure 79 ). 


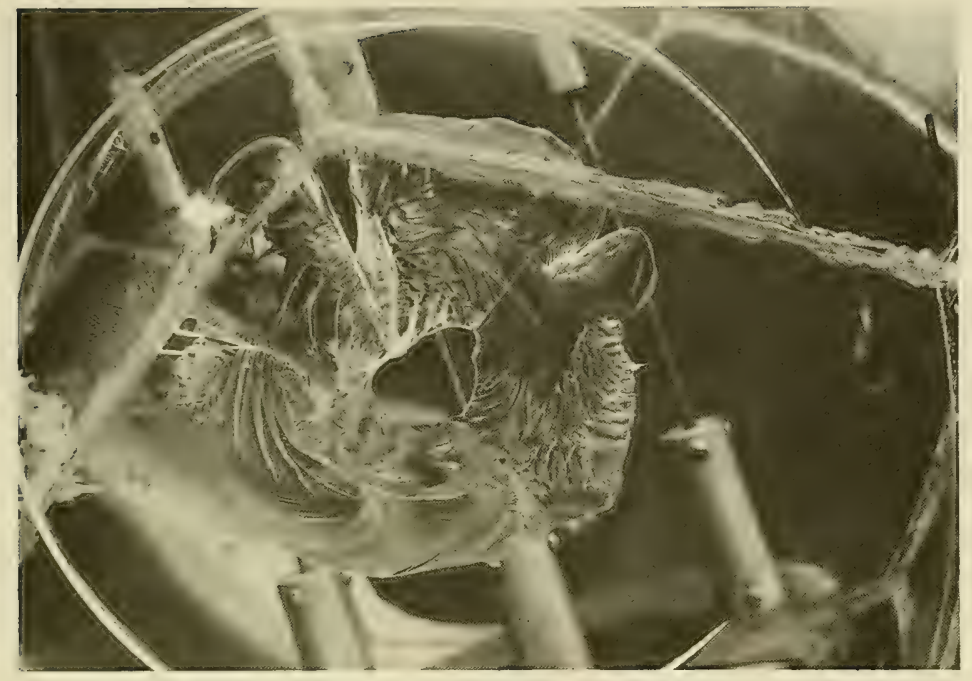

Figure 90. Fracture pattern in a localized viscoelastic capsule failure under sustained hydrostatic pressure.

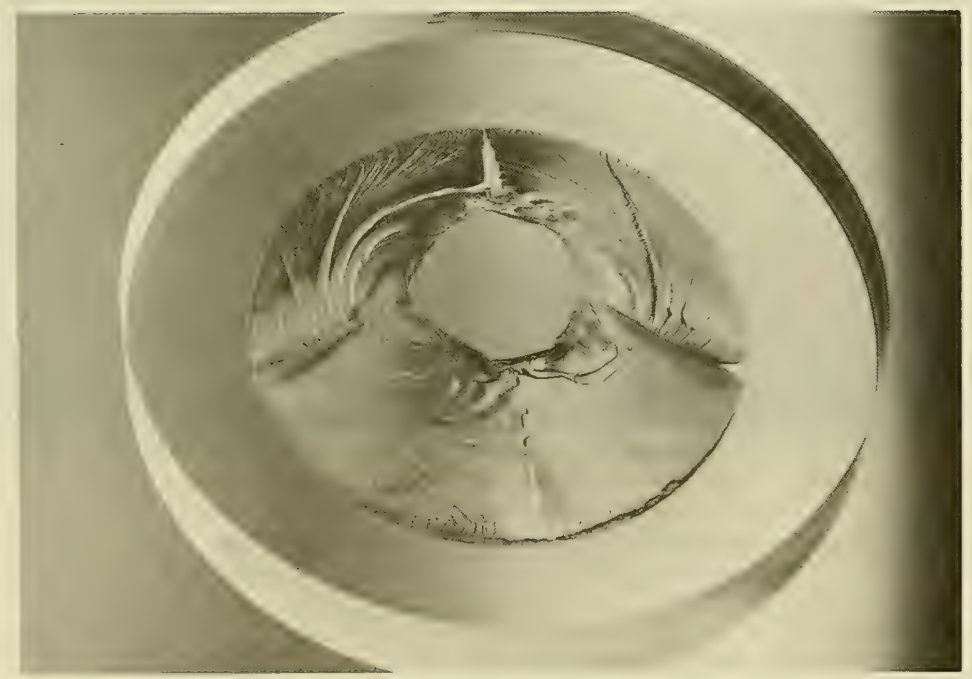

Figure 91. Fracture pattern in a flat disc acrylic plastic window that failed in flexure under short-term hydrostatic loading; high-pressure face. 


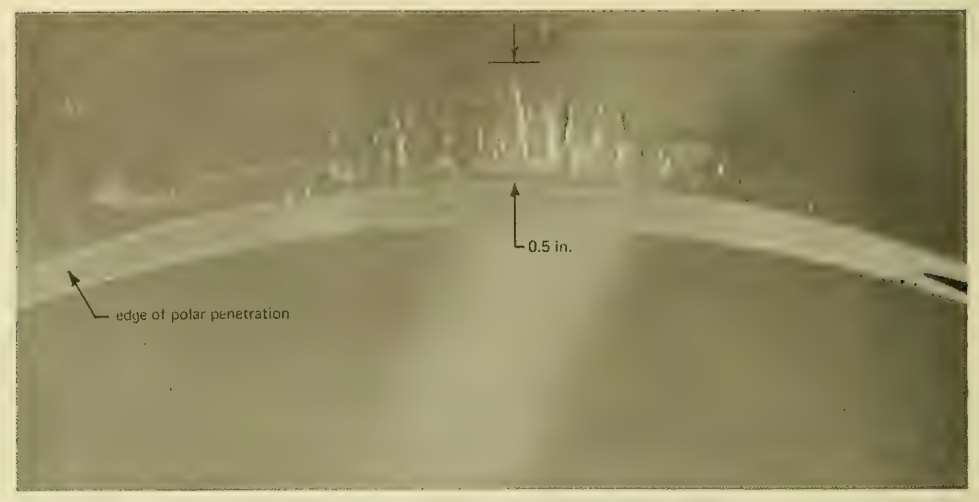

Figure 92. Cracks in beveled edge of acrylic plastic bearing surface for steel insert in a 15 -inch-diameter capsule after 4,500 hours of sustained hydrostatic loading at $750 \mathrm{psi}$ (looking at right angles to the external surface of the capsule).

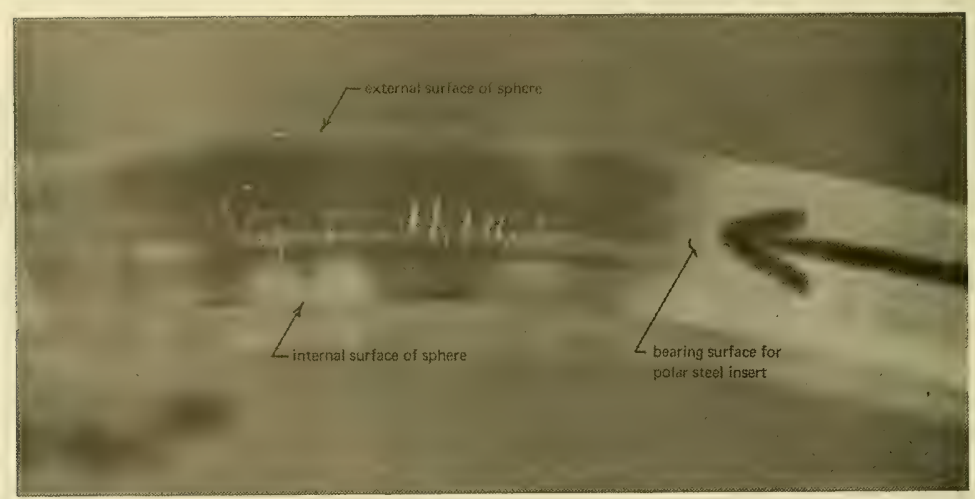

Figure 93. Cracks in beveled edge of acrylic plastic bearing surface for steel insert in a 15-inch-diameter capsule after 4,500 hours of sustained hydrostatic loading at 750 psi (looking at right angles to the beveled bearing surface). 


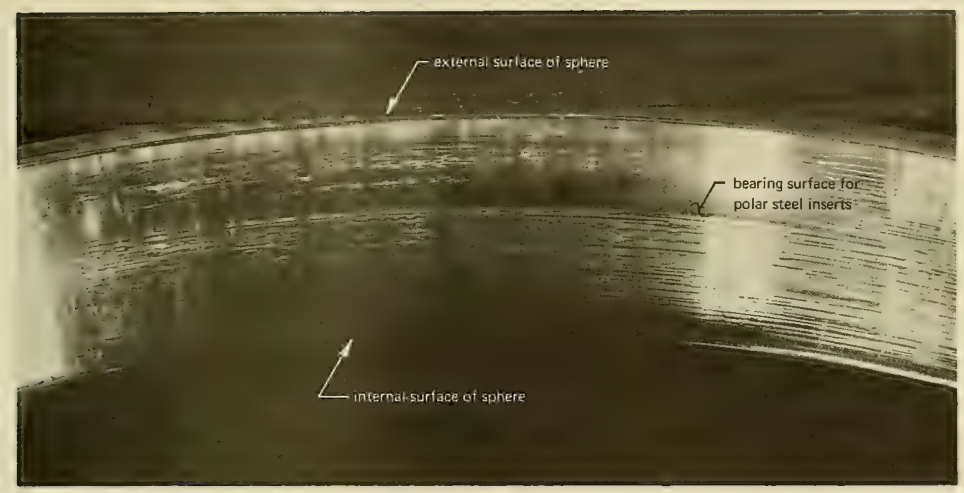

Figure 94. Cracks in beveled edge of acrylic plastic bearing surface for steel insert in a 15-inch-diameter capsule after 72 hours of sustained hydrostatic loading at 900 psi (looking at right angles to the bearing surface).

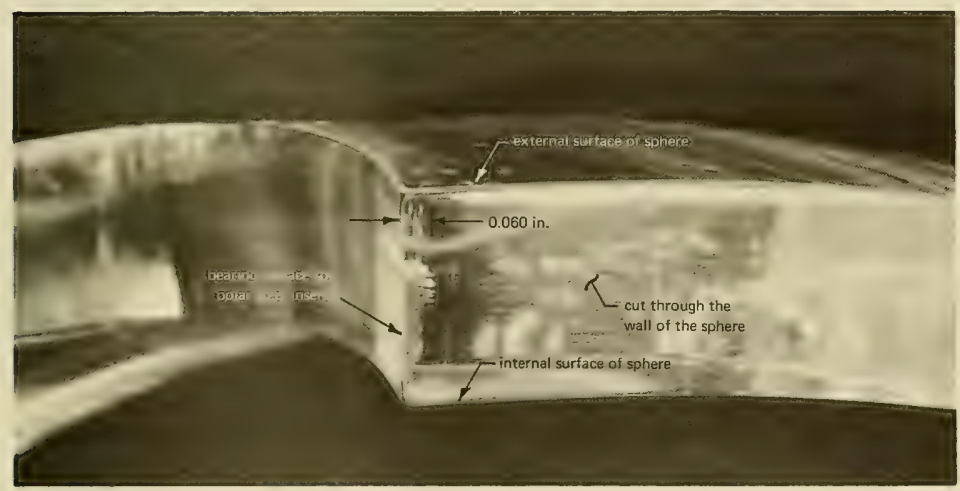

Figure 95. Cracks in the beveled edge of the acrylic plastic bearing surface for steel insert in a 15-inch-diameter capsule after 72 hours of sustained hydrostatic loading at 900 psi (looking at right angles to a cut through the bearing surface). 
This difference can be explained by the hypothesis that since local flat spots and thin sections of the hull cause the acrylic plastic hull to fail at lower pressure by straining locally at a higher viscoplastic rate than the rest of the hull, the rest of the hull does not have the opportunity to reach the compressive strain level at which implosion by general creep buckling mode occurs. Thus, the difference between the volume decrease under short-term and long-term sustained pressure can serve as an indicator of the severity of local deviations from sphericity and thickness in the acrylic plastic hull. The rather small difference between the volume decrease under short-term pressure loading (approximately 1,350 $\mathrm{ml}$ ) and long-term pressure loading (approximately 1,150 $\mathrm{ml}$ ) seems to indicate that the severity of local deviations in sphericity and thickness was rather low. This is, of course, substantiated by actual measurements of sphericity deviations which were on the order of 0.010 to 0.030 inch for the majority of the models.

Cyclic tests were performed on four capsule models to determine the effect of repeated pressurizations on the structural integrity of the acrylic plastic hulls. These tests produced results that are less conclusive than those from short-term and long-term sustained pressure tests, largely because there was only one test specimen for a given test condition and there were only four test conditions (Table 6). Because of these limitations, all the findings address themselves to the effects of three variables only: (1) number of cycles, (2) duration of pressure cycle, and (3) cumulative time under pressure. The effect of pressure magnitude is not discussed because only a single pressure level of 500 psi was used in the cyclic tests.

The cyclic test findings are:

1. None of the model capsules failed during pressure cycling from 0 to 500 psi $(1,120$ feet). The cyclic history at the termination of the tests was 4,000 cycles for the model with a 1-hour sustained loading during the pressure cycle, 600 cycles for the model with a 12-hour loading, 300 cycles for the model with a 24-hour loading, and 100 cycles for the model with a 120-hour loading.

2. No damage was visible in the model capsules except on the beveled acrylic plastic bearing surfaces in contact with the metal end plates. The damage was in the form of circumferential cracks in plane of the wall at right angles to the bearing surface, similar to those observed in some of the capsules under long-term loading. The cracks were concentrated below the inner half edge of the O-ring groove in the steel end plate.

3. By comparing the depth of cracks on the bearing surfaces of all the models cycled from 0 to 500 psi, it has been found that the deepest cracks were found in the model subjected to 100 cycles with a 120-hour dwell time at maximum and minimum pressures. Since the other three models completed 
300,600 , and 4,000 cycles, respectively, the cause for the greater depth of cracks in the model with only 100 cycles could not be the number of stress cycles as otherwise the model with 4,000 cycles would have had the deepest cracks, while in reality it suffered only slightly from cracking (Figure 96).

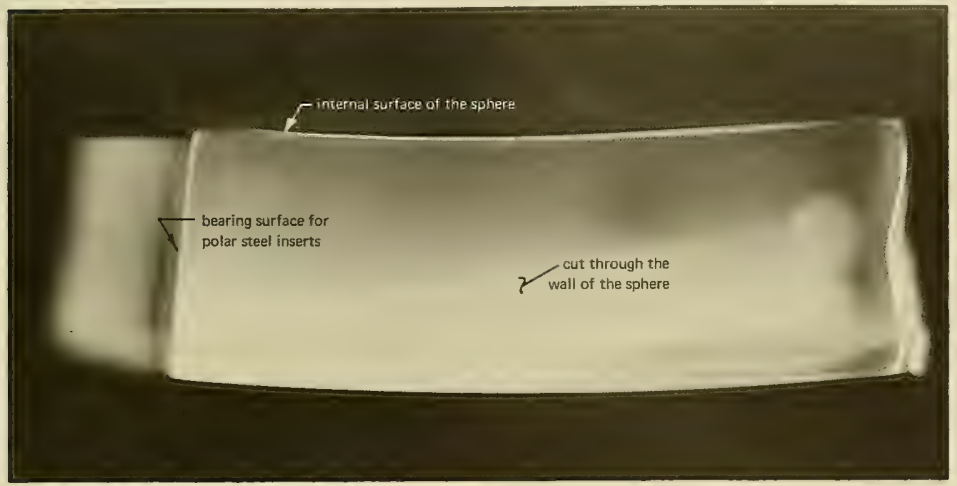

Figure 96. Beveled edge of the acrylic plastic bearing surface for the polar steel insert in a 15 -inch-diameter capsule after 4,000 pressure cycles to 500 psi with 1 -hour dwell times at 500 psi and 0 pressures.

Since the model with the 100 cycles was subjected to the longest duration cycles (120 hours at 500 psi) and had the longest cumulative time under pressure $(12,000$ hours at $500 \mathrm{psi})$ as compared to the others with 24-, $12-$, and 1-hour cycles, respectively, it would appear that in all probability it was either the length of the individual cycle (magnitude of time-dependent strain) or the total cumulative time under pressure rather than the number of cycles that caused this model to have the deepest cracks. It would thus appear that the effect on the acrylic plastic bearing surfaces at penetrations in the capsule of a single long pressure cycle or total cumulative time under load is more severe than the effect of several short cycles of the same pressure level.

Further, it appears that from the two factors (length of individual cycle and cumulative duration of loading at 500 psi) the length of individual cycle is the more important one. This finding is based on the observation that the cracks were more prominent in Model 8 with 300 cycles and 24-hour individual cycle duration (total cumulative time under 500 psi is 7,200 hours) than in Model 11 with 600 cycles and 12-hour duration (total cumulative time of 7,200 hours). Since both models have equal cumulative time under load, while Model 8 has both the longer individual cycle and lesser number of cycles, it could not be either the number of cycles or cumulative time under load that caused it to have more severe cracks than Model 11. 
4. The appearance of cracks in the acrylic plastic bearing surface at the penetrations is not a sign of impending implosion, as none of the models imploded during cycling, although the cracks became visible many cycles before the termination of the cycling program.

5. Pressure cycling capsules to a 500-psi pressure level does not appear to induce irreversible strains in the acrylic plastic, except probably locally on the steel polar closure-acrylic plastic interface. Both the decrease in hull volume, as measured by displacement of water, and the strains on the interior surface of the hull, as measured by electric resistance strain gages, have shown that even after a 120-hour pressure cycle the acrylic plastic hull returns to its original dimensions providing sufficient time is given for its relaxation at 0 pressure. This indicates that both the typical instantaneous and timedependent strains generated in the acrylic plastic hull under a 500-psi loading in the 60 to $70^{\circ} \mathrm{F}$ temperature range are viscoelastic (Figures 97 and 98).

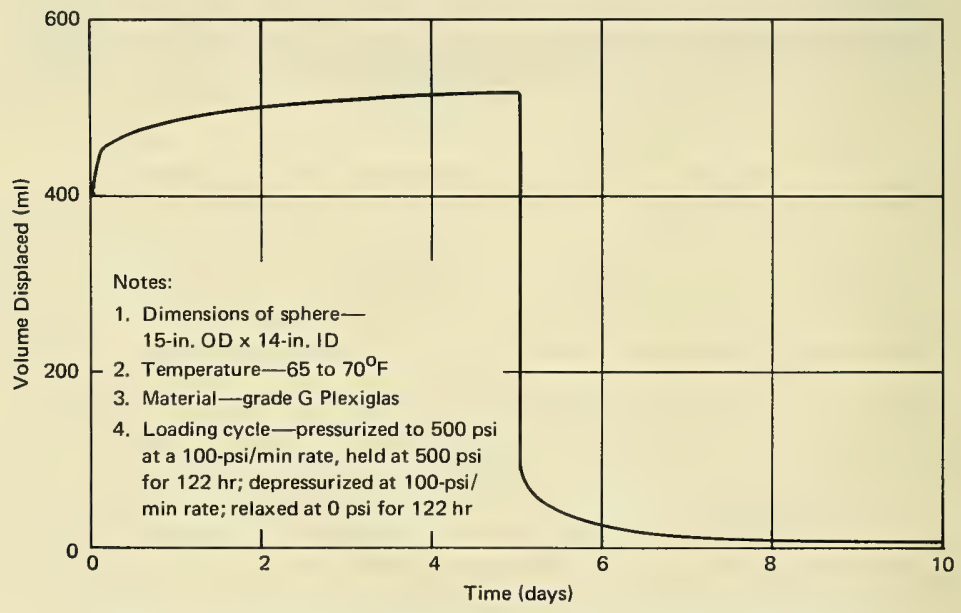

Figure 97. Change in displacement of a 15-inch-diameter capsule during a typical pressure cycle to 500 psi with subsequent relaxation period.

6. The acrylic plastic models were as viscoelastic at the conclusion of their cyclic testing programs as they were at their beginning. This is based on the observation that when the pressure in a given pressure vessel containing a capsule was raised to $520 \mathrm{psi}$ and then the pressurization was terminated, the pressure dropped a certain amount in the isolated vessel during a 24-hour period due to a decrease in volume of the capsule. Since the decrease of 
pressure in an isolated pressure vessel in a 24-hour period during the first pressure cycle was found to be equal to the pressure drop recorded in the same vessel during some later cycle, the decrease in volume of the capsule model was the same in both cases. For example: the pressure drop in 24 hours during the first pressure cycle on Model 8 was from 520 psi to 453 psi. Subsequently, the pressure drop was measured again during the 234th cycle and was also found to be from 520 to 453 psi.

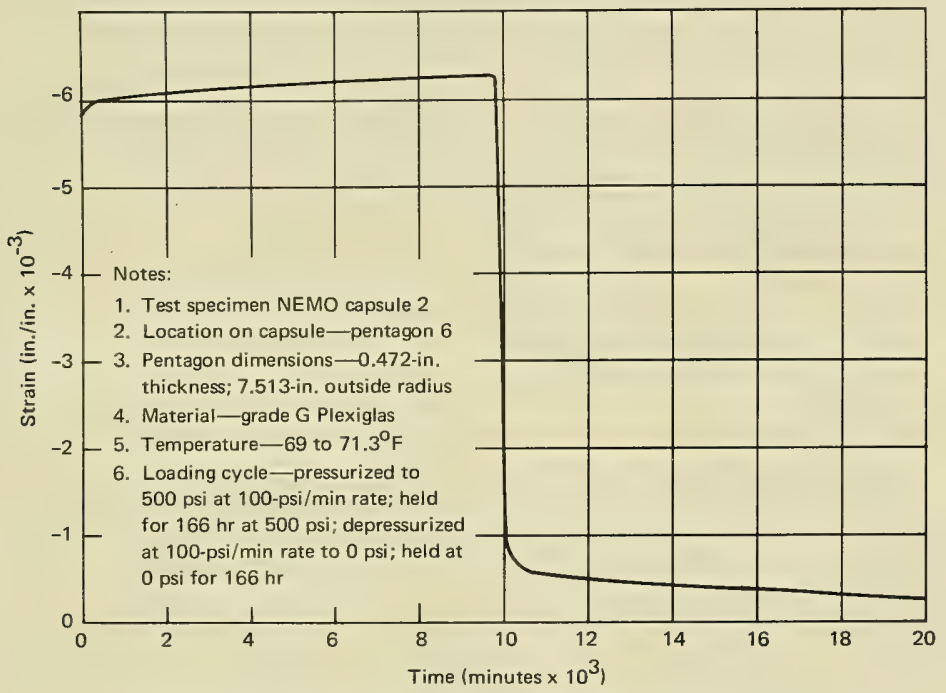

Figure 98. Typical viscoelastic strain on the interior of a 15-inch-diameter capsule in the equatorial region during a typical pressure cycle to 500 psi with subsequent relaxation period.

An axial compression test conducted on a single model capsule to simulate tie rod loadings produced only exploratory data. Since the electric resistance strain gages, located both on the exterior and interior of the hull, covered only a small area of the surface, a complete picture of the stress field in the acrylic plastic hull under axial compression was not obtained. The load was applied to the hull by internal tie rods attached to the circumference of the steel end plates. On the basis of this data.(Figures 99 and 100), it has been found that:

1. The stresses generated by axial tie-rod pretension of 100 pounds are higher at the steel polar plate than at the equator. The meridional stresses at the penetration show compression on the exterior and tension on the interior. 
The hoop stresses at the polar plate also show compression on the exterior surface of the hull and tension on the interior. The hoop stresses, both on the exterior and the interior, are smaller than meridional stresses.

2. The stresses at the equator of the acrylic plastic sphere were barely noticeable. The meridional stresses showed compression both on the outside and on the inside, while hoop stresses showed tension both on the outside and on the inside. The compressive meridional stress was higher on the inside than on the outside surface. The tensile hoop stresses were approximately equal for the outside and inside surfaces of the hull.

3. The tensile stresses caused by pretensioning of tie rods are so small as to constitute a negligible effect on the distribution of compressive stresses superimposed during hydrostatic pressure tests.

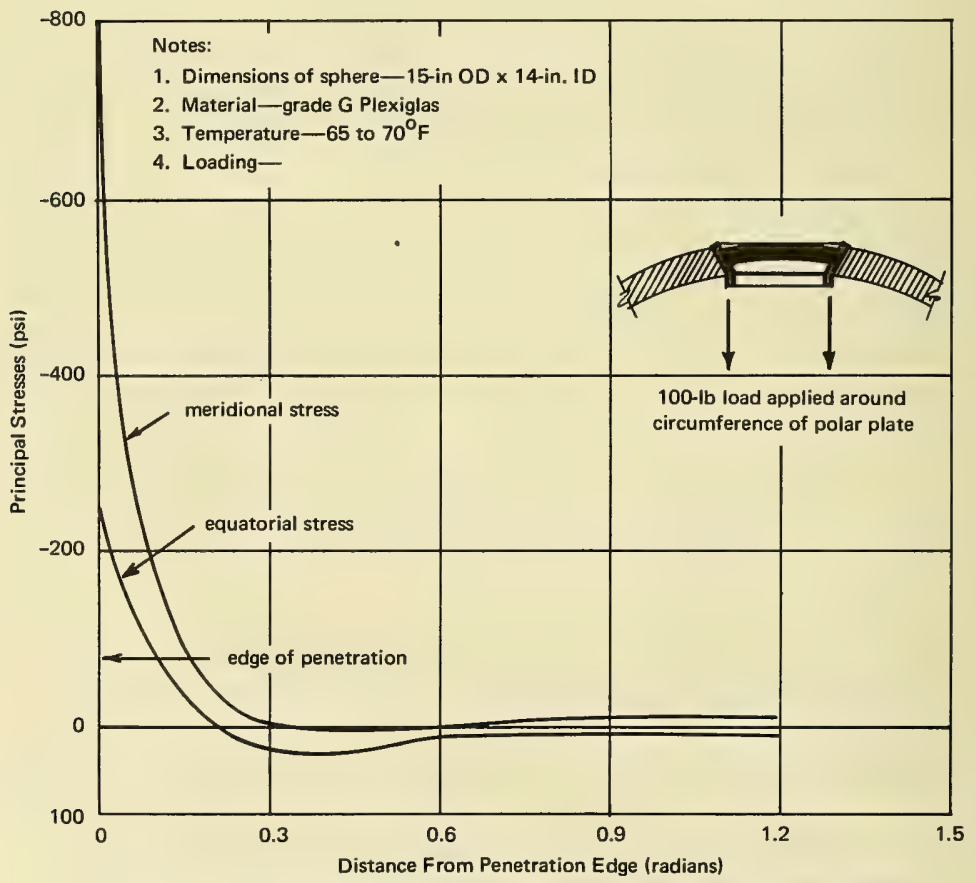

Figure 99. Stresses on the exterior of a 15-inch-diameter capsule under axial compression applied by internally located pretensioned tie rods. 


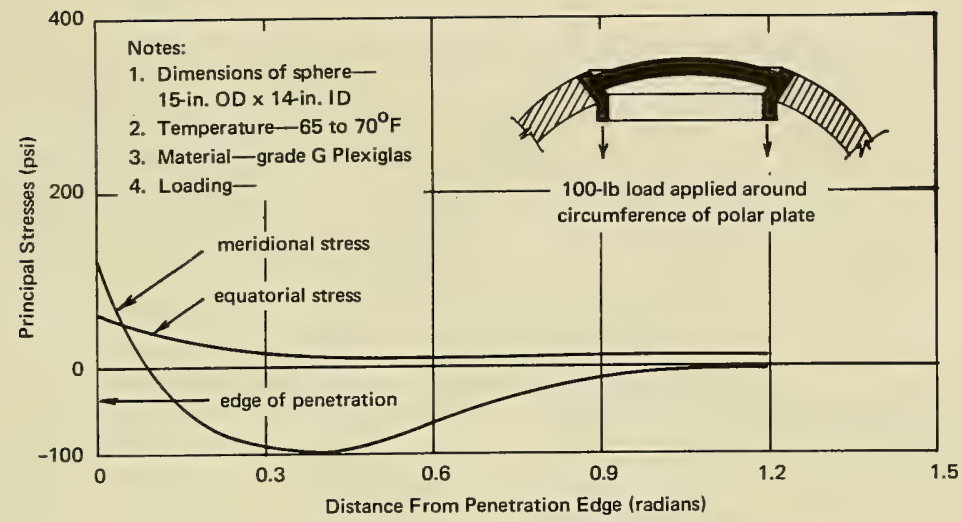

Figure 100. Stresses on the interior of a 15-inch-diameter capsule under axial compression applied by internally located pretensioned tie rods.

\section{Large-Scale Capsule}

Because only one 66-inch-OD x 61-inch-ID large-scale capsule prototype (NEMO capsule 0) was available for the determination* of the many facets of structural behavior exhibited by the acrylic plastic hull, it was much more exhaustively instrumented and produced considerably more data than the model capsules. This is particularly true of the strain data generated by 81 strain gages (Figure 101) during more than 100 pressure cycles of different periods and pressure magnitudes. Since the detailed evaluation of this voluminous strain data and its comparison with computer generated values ${ }^{21}$ is a rather involved operation, the detailed discussion of experimental data were omitted from this report to be published as a separate report at a later date. ${ }^{22}$ However, in order to make this report complete, the findings of the strain and stress analysis are presented here in the form of summaries (Figures 102 and 103); there are also discussions of tests that did not employ strain gage instrumentation systems.

Since all the tests performed on the large-scale capsule prototype were cyclic, even though of different period duration and maximum pressure level, the discussion of findings will be classified by type of response or physical characteristic rather than by test as was done in the discussion of findings for model capsules.

* The proof tests performed on the three production 66-inch NEMO capsules were very limited and therefore are not discussed in the main body of the report. (See Appendix F.) 
(80)

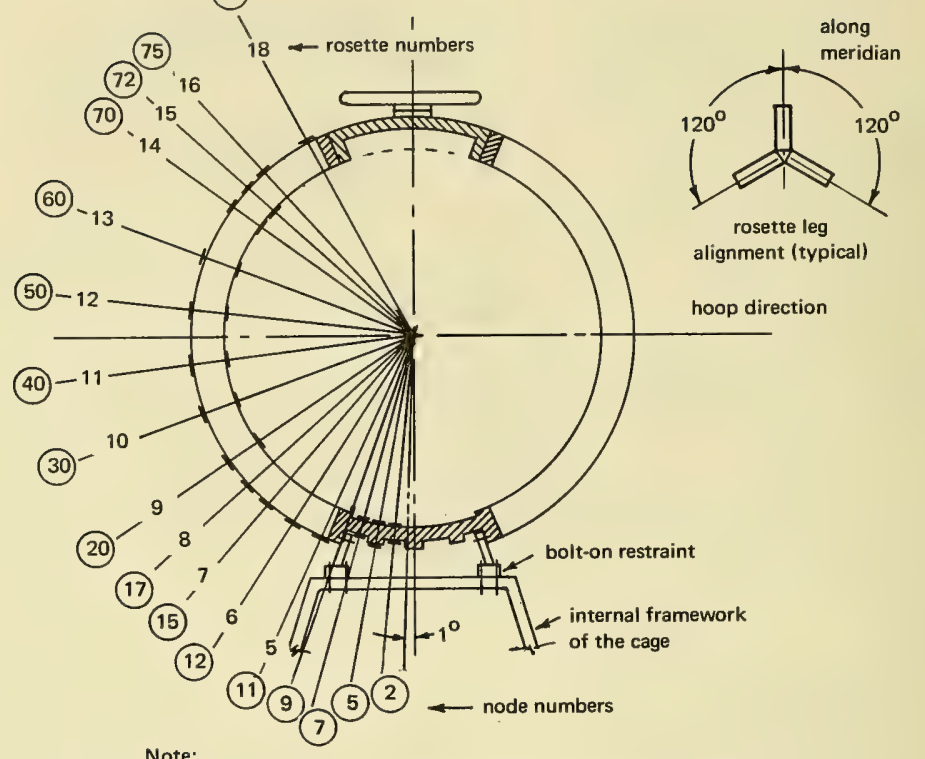

Note:

Node locations have been determined by dividing the sphere into 2-degree increments. Numbering of nodes began on the bottom, just as the numbering of the rosettes, and continued to the top.

Figure 101. Location of strain gages on the 66-inch-diameter capsule for external pressure hydrostatic testing program.

Short-term strains and stresses in the large-scale capsule prototype were recorded at 27 locations (Figure 101) during all the pressurization in the 0-to-800-psi pressure range. Strains were also recorded at two locations during the differential temperature tests. The findings, based on strain gage recordings during the short-term pressurization from 0-to-500-psi pressure are:

1. The short-term strains in the acrylic plastic hull varied linearly with pressure (Figure 104). The maximum principal strains on the interior surface in the equatorial regions were approximately $20 \%$ higher than on the exterior surface of the hull. The magnitude of the principal strains on the interior surface of the equator at 500 -psi short-term pressure and $68^{\circ} \mathrm{F}$ 
temperature was in the region of 4,500 to $5,100 \mu \mathrm{in}$./in., while those on the exterior were in the 3,500 to $4,100-\mu \mathrm{in}$./in. range. The exact magnitude and distribution of strains in the acrylic plastic around the polar plates is not known because they were not instrumented in sufficient detail; there are indications that the ratios of strains at the polar plates to those at the equator were lower than those measured on the model. This was probably caused by the difference in polar plate fastening procedures used for the model and for the 66-inch capsule. While in the model the pretensioned tie rod seated the end plate with only a 100-pound axial force in the 66-inch capsule the polar plate was seated in the polar opening with approximately 100,000 pound force generated by 20 bolts reacting against the split retaining flanges. The very large seating force pretensioned the acrylic plastic around the polar plate to such an extent that only minor compressive strains could appear there during hydrostatic testing.

2. The strains in the steel bottom plate also varied linearly with pressure. The strains measured on the bottom steel plate varied from one location to another with the strains in one location approaching the yield point of type 316 stainless steel from which the end plates were made. The gage that measured the highest strain on the steel polar plate was rosette 3 located on the interior surface of the bottom plate in the immediate vicinity of the plate flange.

3. When the measured strains at 500-psi short-term pressurization and $68^{\circ} \mathrm{F}$ were converted into stresses, the magnitude of membrane stresses at the capsule's equator was found to be approximately 3,400 psi on the interior and 2,900 psi on the exterior. The highest measured stress in the steel end plate at rosette 3 was 27,000 psi (Figures 101 and 103).

Short-term volume decrease, measured with the same type of volumedisplacement arrangement as in the model capsules, at $68^{\circ} \mathrm{F}$ was linear to $750 \mathrm{psi}$, the maximum pressure to which the sphere was pressurized in that test. The volume decrease was approximately 5.5 liters per each $100 \mathrm{psi}$ of short-term pressure increase (Figure 105). When proper scaling factors were applied to the measured volume decrease of the prototype large-scale capsule, it was found to be the same as for the model capsules under shortterm loading (Figure 106). Two separate scaling factors were employed. One factor accounts for the fact that the volume of a spherical hull varies as the cube of the radius. The other factor accounts for the fact that the actual wall thickness of the large-scale capsule was somewhat disproportionately thicker than that of the model capsule. 


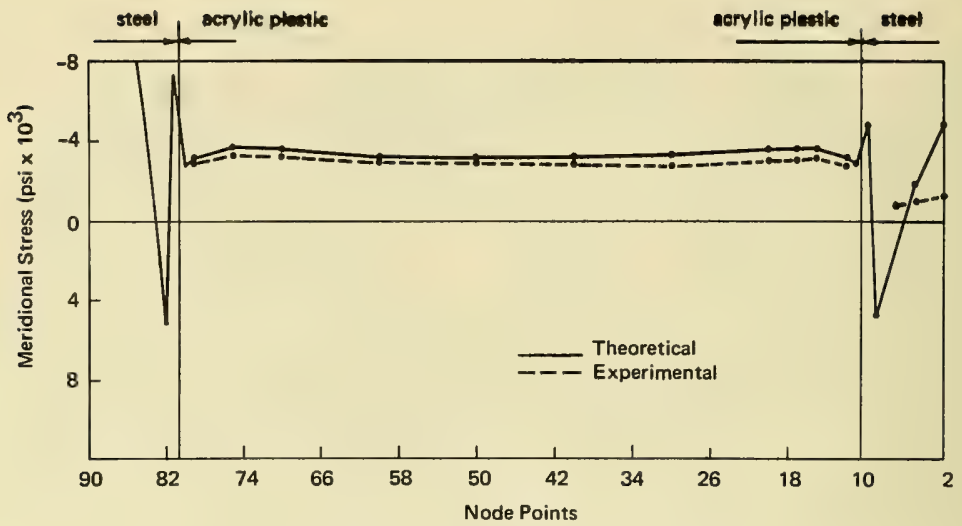

(a) Meridional stresses on the exterior surface.

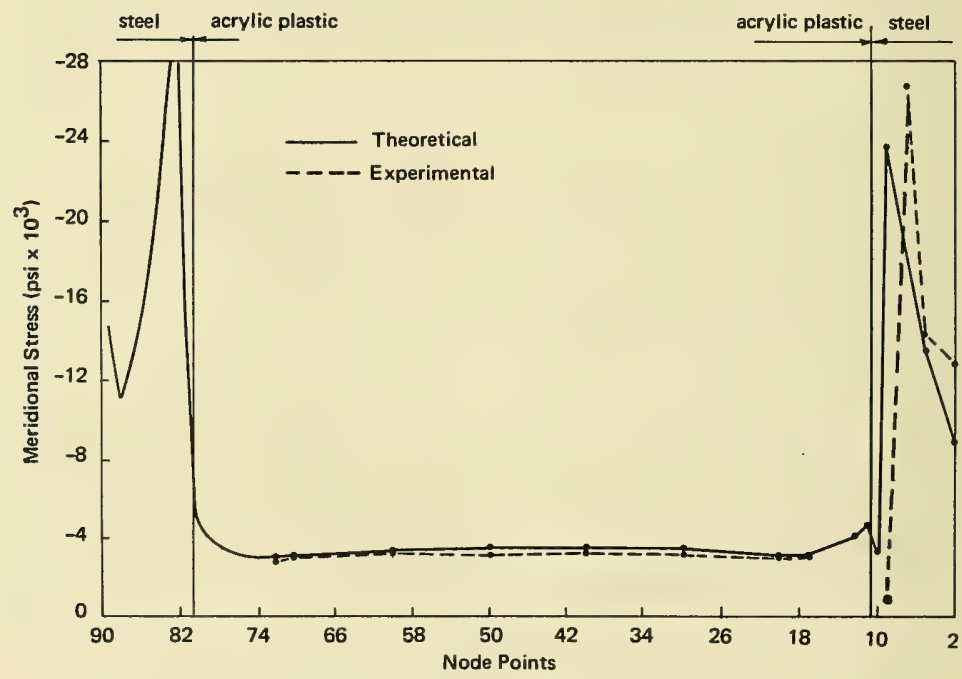

(b) Meridional stresses on the interior surface.

Figure 102. Comparison of analytically and experimentally derived stresses in the 66-inch-diameter capsule under 500-psi short-term hydrostatic loading. 


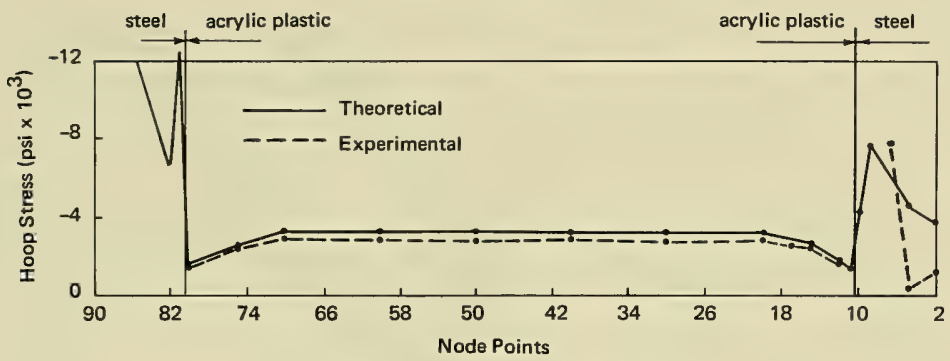

(a) Hoop stresses on the exterior surface.

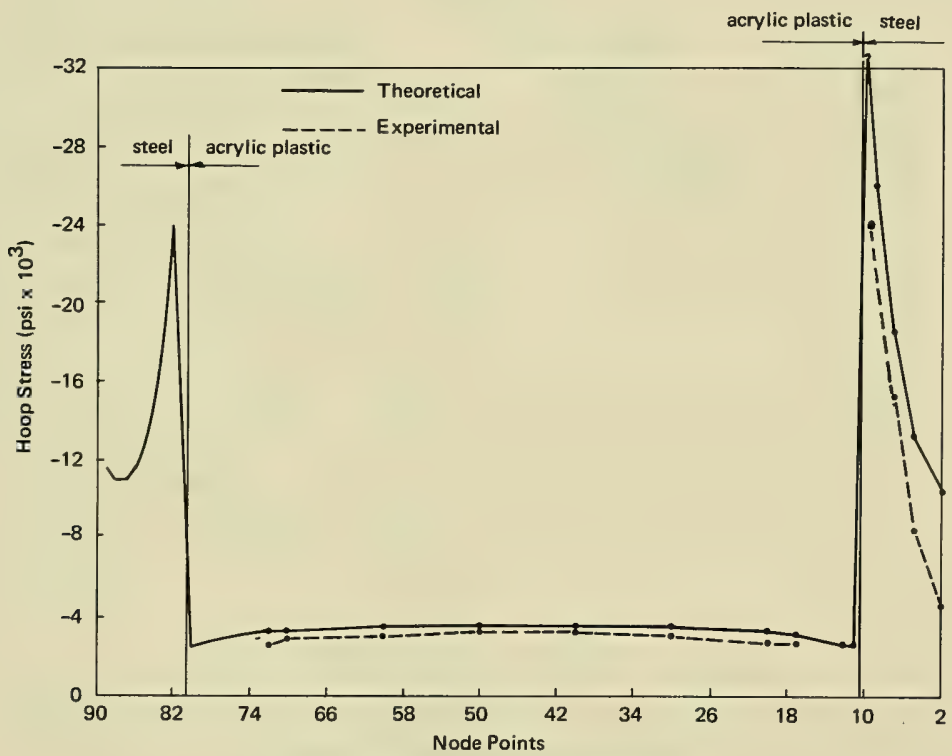

(b) Hoop stresses on the interior surface.

Figure 103. Comparison of analytically and experimentally derived stresses in 66-inch-diameter capsule under 500-psi short-term hydrostatic loading. 


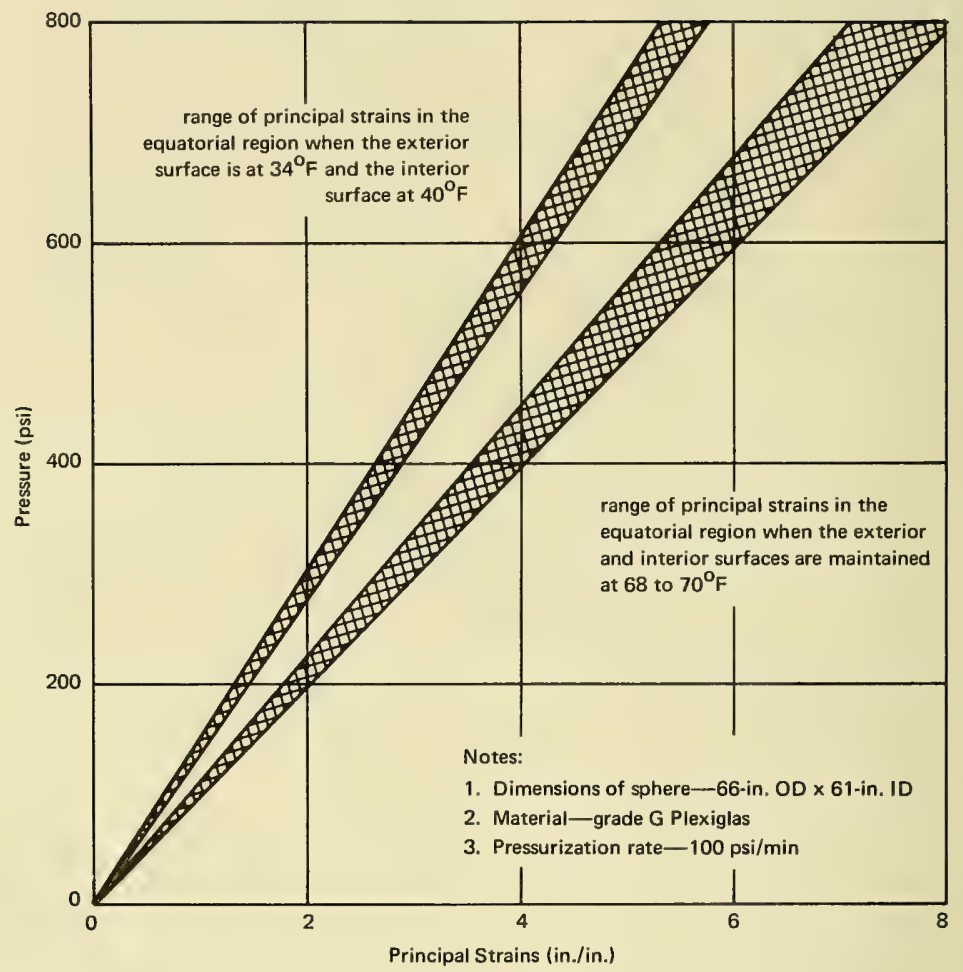

Figure 104. Effect of ambient temperature on strains in 66-inch-diameter capsule under short-term loading: typical strain on interior surface in the equatorial region.

Cyclic strains generated by pressure cycling were recorded at 36 locations on the acrylic plastic hull. Since all the pressure cycles had 500 psi as the upper pressure limit, the following findings pertain only to pressure cycling of that magnitude.

1. The principal strains measured at the equator on the external and internal surfaces of the hull during the pressure rise in each hydrostatic cycle were found to be in same range as those measured during a single short-term loading. 


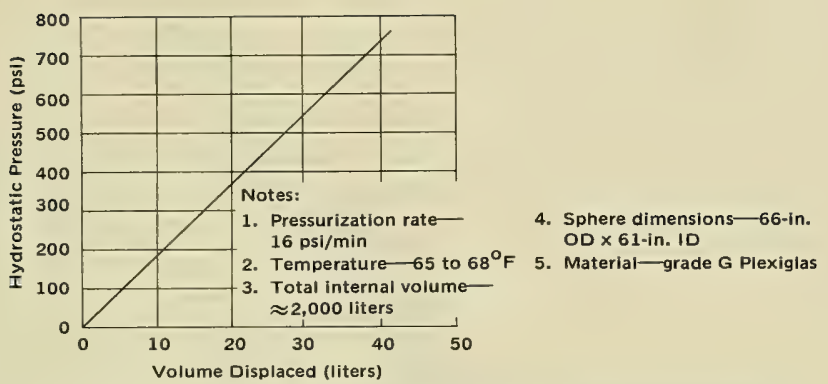

Figure 105. Change in displacement of 66-inch-diameter capsule under short-term loading.

2. The relaxation periods at zero pressure, which were chosen for all pressure cycles to be equal in length or longer than the duration of sustained pressure periods, appeared to be sufficiently long for all strains to return to zero (Figure 107). Thus, the magnitude of strains in acrylic at the initiation of each pressure cycle rose by the last cycle from zero to only $100 \mu \mathrm{in} . / \mathrm{in}$. This took place regardless of whether the length of each cycle was 2,15 or 60 minutes.

Long-term strains were also recorded at 27 locations while the hull was subjected to long-term loading of 24-hour duration at 100-, 200-, 300-, 400-, 500-, 600-, 700-, and 800-psi external hydrostatic pressure. Since the relaxation periods were sufficiently long between each long-term pressurization to allow for a complete relaxation of the hull, the recorded strain data was not considered to be contaminated by viscoelastic strains still undergoing relaxation when the next pressure cycle was initiated.

1. At room temperature, the magnitude of strains in the equatorial region under long-term hydrostatic loading after a given duration of sustained loading appears to vary linearly with pressure. To what maximum pressure this relationship remains linear is not known, as the long-term pressure tests were conducted only to $800 \mathrm{psi}$, at which pressure the relationship still continued to be linear (Figures 108, 109, 110).

2. Upon depressurization, the strains in the equatorial region returned almost to zero, even after long-term 800 -psi pressure loading. This appears to indicate that even at an 800-psi long-term hydrostatic loading in the 68 to $70^{\circ} \mathrm{F}$ temperature range, the strains in the capsule (with possible exception of polar regions) are probably still viscoelastic (Figures 109 and 110). 


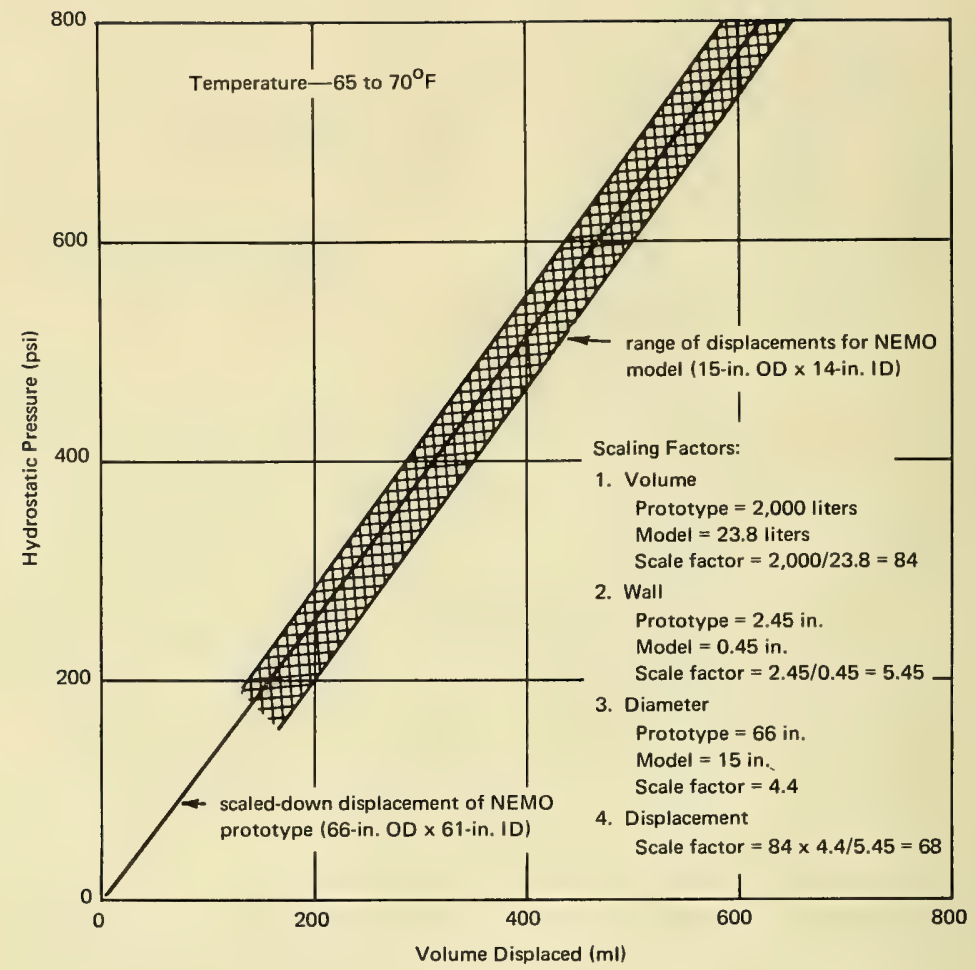

Figure 106. Comparison of displacement change measured on 66-inch-diameter capsule and predicted displacement based on extrapolation of displacement change data from 15-inch-diameter capsule under short-term loading.

3. The time-dependent strain rate varies with the pressure to which the acrylic plastic hull is subjected during long-term loading. The relationship between the time-dependent strain rate and pressure is probably exponential.

4. Immediately after initiation of long-term pressure loading, the strain and time-dependent strain rate were higher on the interior of the hull in equatorial region than on the exterior of the hull. This was caused by the presence of higher stresses on the interior of the hull at that time. (Figures 109 and 110 . 

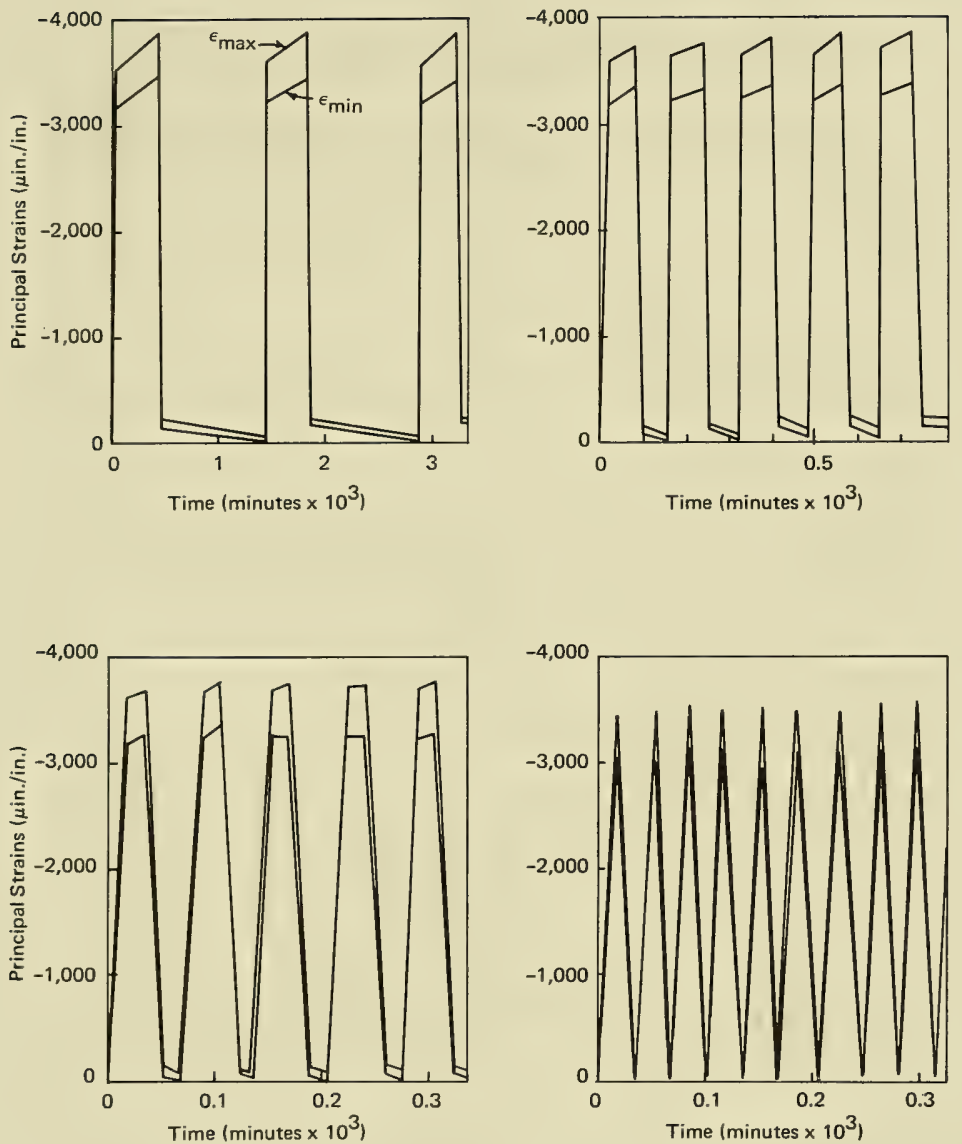

Figure 107. Strains on the interior surface of 66-inch acrylic plastic capsule under cyclic pressure loading to 500 psi. 


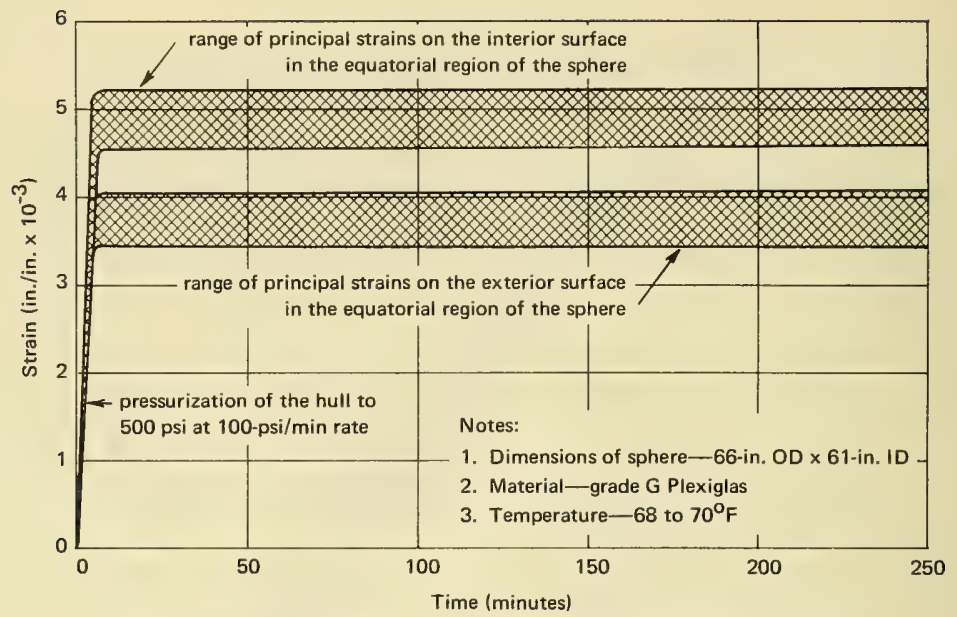

Figure 108. Range of strains at the equatorial region of the 66-inch-diameter capsule under sustained hydrostatic loading of 500 psi.

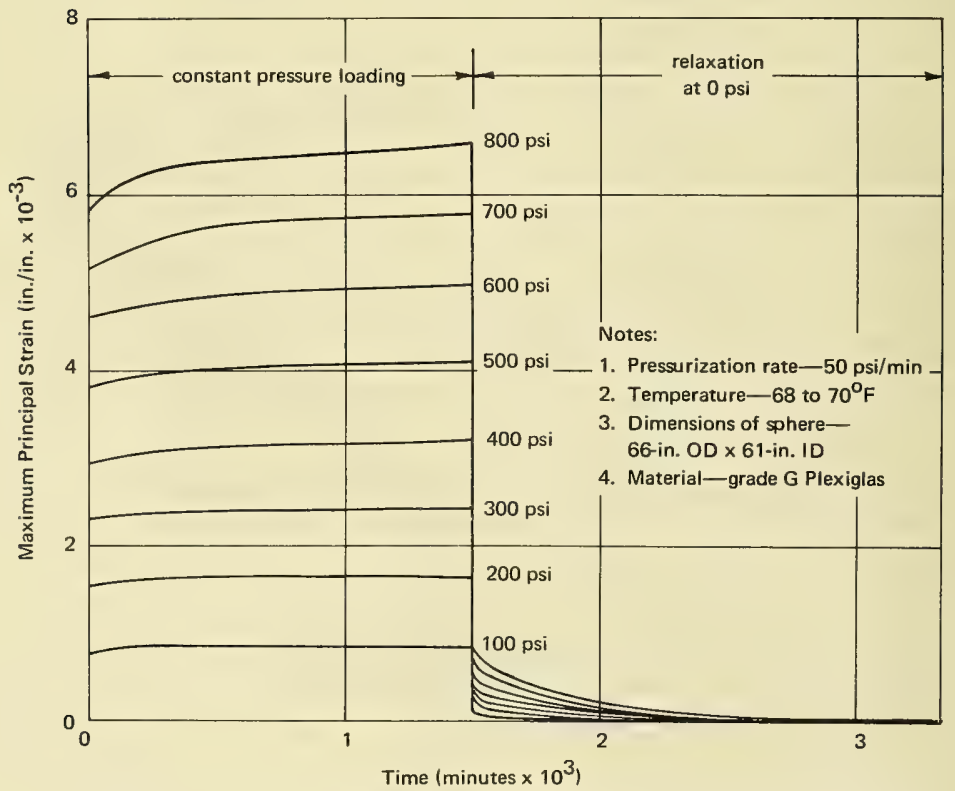

Figure 109. Typical viscoelastic strain on exterior surface of equatorial region in 66-inch-diameter capsule under sustained hydrostatic loadings of different magnitude, and subsequent relaxation. 


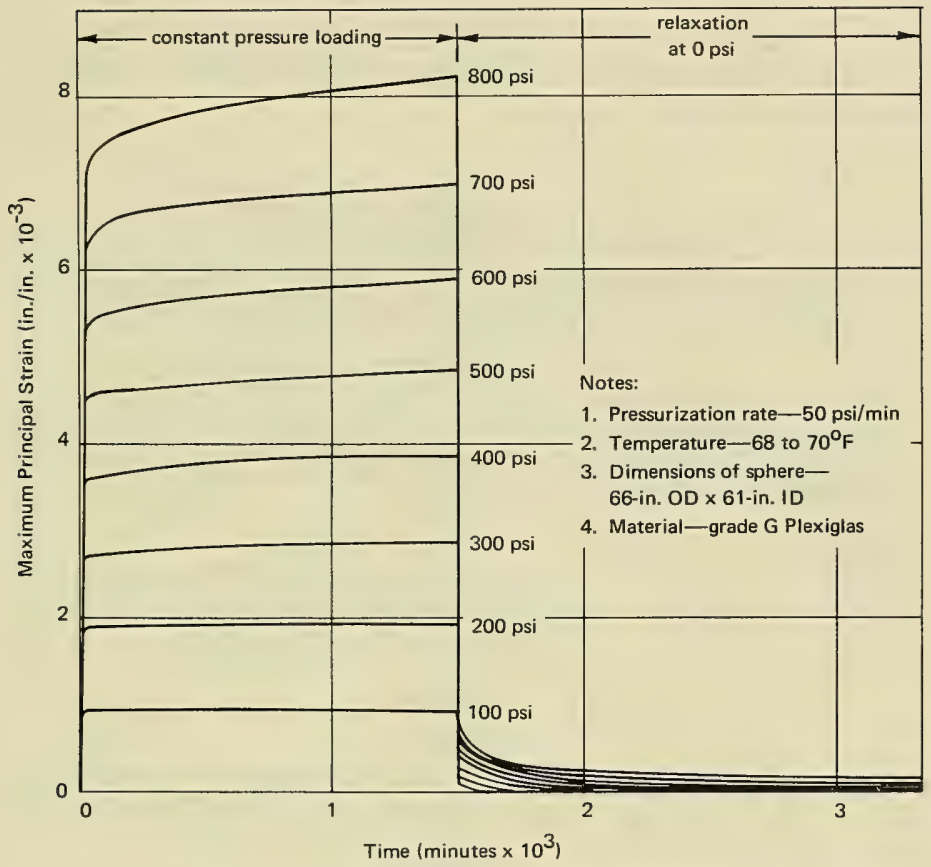

Figure 110. Typical viscoelastic strain on interior surface of equatorial region in the 66-inch-diameter capsule under sustained hydrostatic loadings of different magnitude and subsequent relaxation.

5. The strain, as well as the time-dependent strain rate, vary with the temperature of the pressurizing medium. Comparison of strains and strain rates in 32 to $35^{\circ} \mathrm{F}$ temperature range with those in the 68 to $70^{\circ} \mathrm{F}$ range shows that in the low temperature range they are smaller by about $20 \%$ to $30 \%$ (Figure 111).

6. If the acrylic plastic capsule is depressurized to some pressure level above 0 psi, the strains will initially begin to relax as they do when the external pressure level is reduced to $0 \mathrm{psi}$. After a period of time, the time-dependent relaxation of strains will cease and time-dependent increase in strain will begin, but the rate will be lower than for hulls not previously loaded to higher pressure levels. However, after a sufficiently long period of time, the strains reach the same equilibrium point regardless of whether the hull was first loaded to some overpressure and then depressurized to the operational pressure level or the pressure was raised to the desired level directly from 0 psi. Upon depressurization to operational pressure, the time required for the strains resulting 
from overpressure to decrease to the level of strains generated by a simple long-term loading at operational pressure varies with the duration of loading at the overpressure level (Figure 112).

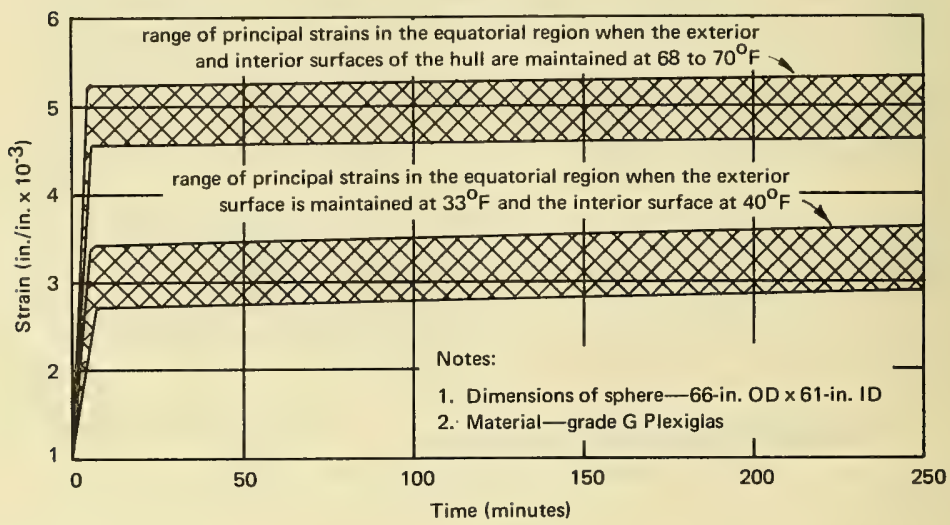

Figure 111. Effect of temperature on viscoelastic strains on interior surface of equatorial region in 66-inch-diameter capsule under 500-psi sustained hydrostatic loading.

Long-term volume decrease, measured by the displacement of water from the interior of the large-scale capsule, was performed only at three different pressure loadings, as compared to nine different pressure loadings for model capsules. From the volume decrease measurements performed on large-scale capsule prototype, the following findings were made:

1. Long-term volume decrease is a function of both time and pressure. However, for any given duration of loading, it is approximately linear with the magnitude of pressure loading. The time-dependent rate of volume decrease for any given pressure loading appears to decrease exponentially with time, and to increase exponentially with the magnitude of pressure loading (Figures 113 and 114).

2. Upon depressurization from the long-term pressure loading, the volume of the large-scale prototype capsule returned to its original size after a relaxation period of approximately the same duration as the long-term pressure loading. The volume increase rates at the beginning of relaxation periods were higher than the volume decrease rates at the beginning of the long-term loading periods (Figure 115). 


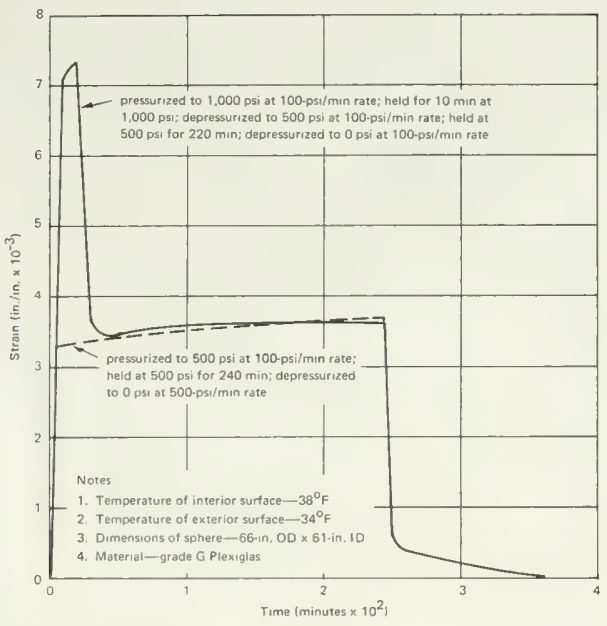

Figure 112. Comparison of strains under 500-psi sustained hydrostatic loading and strains under 500-psi sustained hydrostatic loading following a sustained loading of 1,000 psi; strains were measured on the interior surface in the equatorial region of the 66 -inch-diameter capsule.

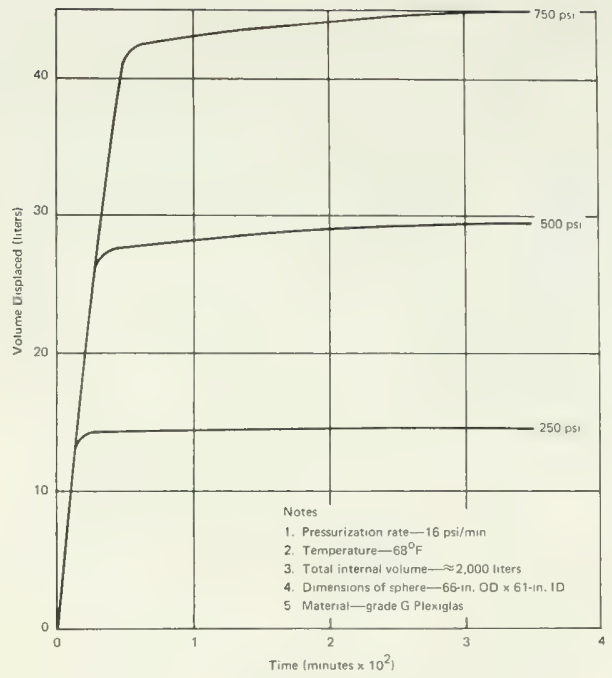

Figure 113. Change in displacement of the 66-inch-diameter capsule under sustained hydrostatic loadings of different magnitudes.

147 


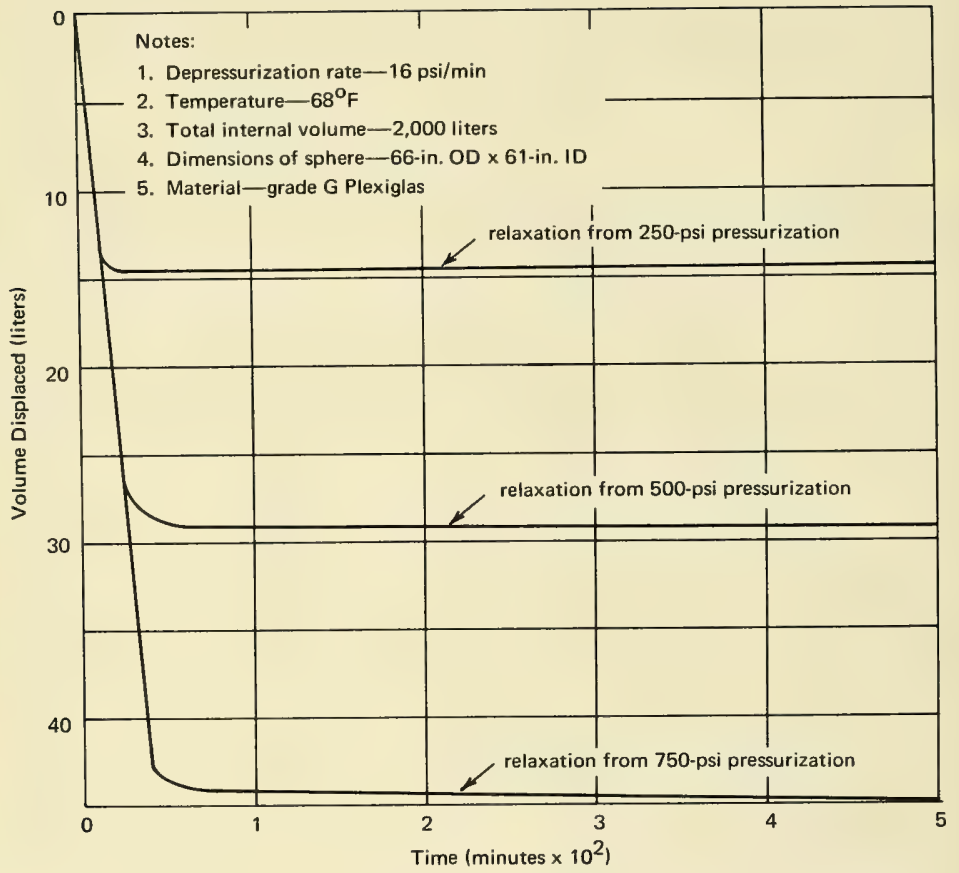

Figure 115. Change in displacement of the 66-inch-diameter capsule during relaxation from sustained hydrostatic loadings of different magnitude. 
Short-term strength of the large-scale acrylic plastic capsule was determined by pressurizing it externally to implosion at a $100 \mathrm{psi} / \mathrm{min}$ rate with water at $70^{\circ} \mathrm{F}$ ambient temperature. The implosion took place at 1,850 psi with a failure mechanism similar, if not identical, to that of the models previously imploded under short-term loading. From the observation of the fragments from the imploded sphere as well as comparison of the failure pressure of the large capsule and model capsules the following findings were made:

1. There appears to be no material mass scaling effect, common to glass or concrete structures, in acrylic plastic since the 1,850-psi implosion pressure of the 66-inch capsule matches closely the 1,827-psi pressure predicted for the large-scale capsule on the basis of model capsule tests. This prediction is based on the 1,575-psi average implosion pressure of the 15-inch acrylic plastic models and the fact that the minimum wall thickness of the 66 -inch capsule is $16 \%$ greater than the minimum thickness of the models.

2. The fragmentation of the large-scale capsule was similar to that of models (Figure 116). In most cases, the fracture planes cross the joint bonds indicating that the joints did not serve as planes of weakness.

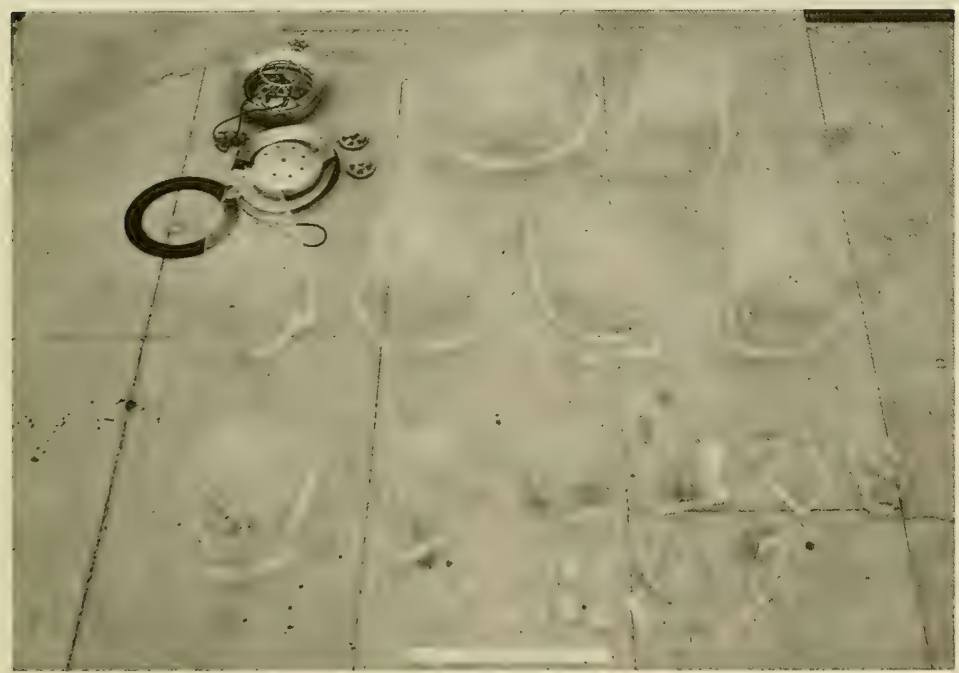

Figure 116. Overview of the structural fragments from the imploded 66-inch-diameter capsule. Note that the acrylic plastic fragments are not rectilinear, indicating that fracture did not take place along the bonded joints. 
3. All of the steel end closure components except for the top hatch ring and associated split retaining rings showed extensive plastic deformation (Figure 117) indicating that the large-scale polar steel closures underwent plastic buckling at approximately the same pressure as the model scale steel closures which failed at 1,800 psi (Figure 80). This validates the linear scaling factor (diameter of prototype capsule/diameter of model capsule) used in the scaling up of model capsule end plates for use in the 66-inch prototype NEMO capsule.

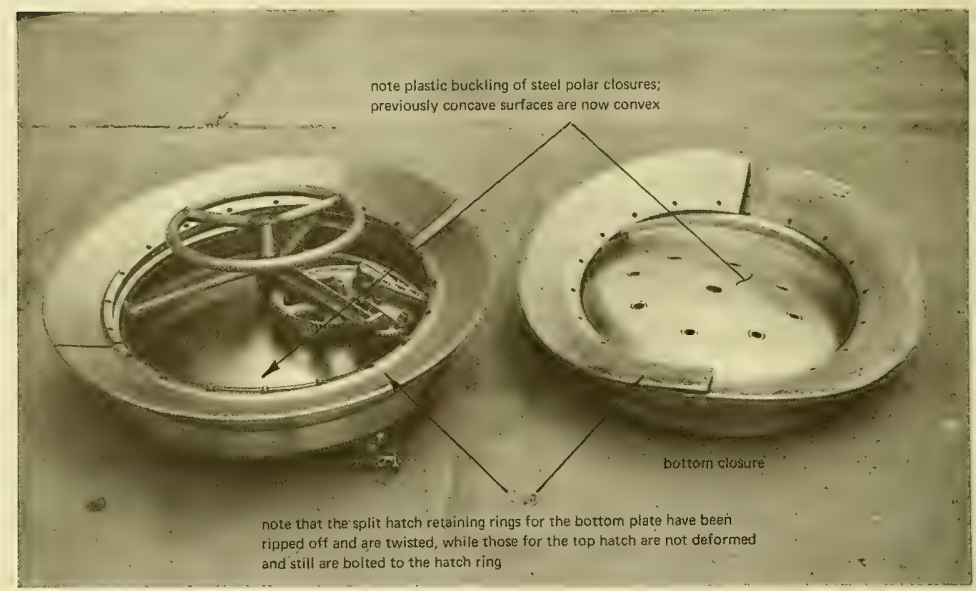

Figure 117. Steel polar closure assemblies from the imploded 66-inch-diameter capsule.

4. The general implosion of the prototype NEMO capsule was triggered by the plastic buckling of the bottom plate. This finding is supported by the fact that (a) only on the bottom plate were the hatch retaining rings twisted, (b) only on the bottom plate was the spherical beveled bearing surface severely distorted, (c) only the bottom polar pentagon had its beveled bearing surface severely damaged (Figures 118 and 119), (d) rosette 3 mounted at the location where plastic hinge in the bottom plate formed showed the highest stress level during previous tests to $500 \mathrm{psi}$.

5. Since the buckling of the hatch and of the bottom plate was plastic rather than elastic, it can be avoided at pressures less than 2,000 psi by substituting material with a yield point above 60,000 psi for the type 316 stainless steel used in the prototype. 
Structural fatigue of the 66-inch hull was evaluated visually by noting the condition of bearing surfaces on the beveled edges of the acrylic plastic hull at the polar penetrations and the condition of the bonded joints between individual spherical pentagons at the conclusion of 107 pressure cycles. The maximum pressure in the individual pressure cycles varied from 100 to 1,070 psi and its duration from 1 minute to 24 hours.

1. Bonded joints did not have any indications of fatigue damage, although the joints had a large number of potential stress raisers in the form of cavities, separation spaces, and hairline cracks introduced during fabrication.

2. The bearing surface on the beveled edge of the polar penetrations had no indications of fatigue in the form of crazing, even though during the preceding 107 pressure cycles the hydrostatic pressure loading was in some cycles 2.4 times higher than pressure at the design depth of 1,000 feet ( 4 times higher than pressure at the operational depth of 600 feet).

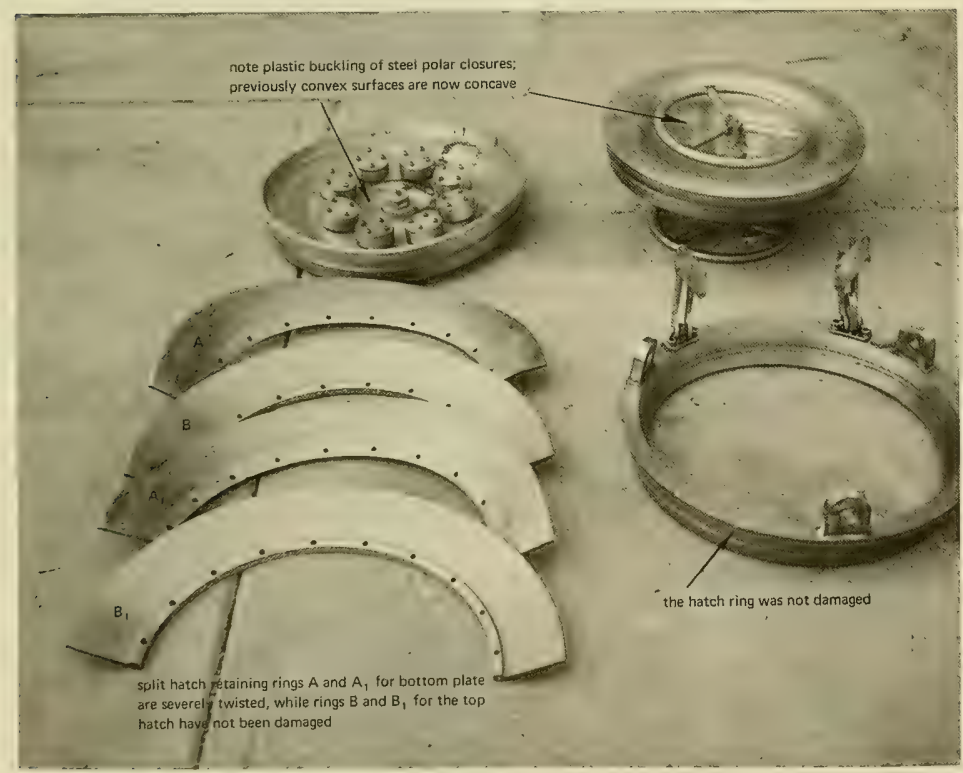

Figure 118. Steel polar closure components from the imploded 66-inch-diameter capsule. 
3. The short-term test to failure at conclusion of 107 pressure cycles caused the capsule to fail at $1,850 \mathrm{psi}$. The actual implosion pressure of the large-scale capsule matches the implosion pressure of 1,827 psi predicted on the basis of implosion tests on model capsules. Therefore, it can be postulated that no decrease in structural strength occurred as result of the previous pressure cycling to which the capsule was submitted.

Heat transfer capability of the large-scale capsule was established in a stagnant water environment with a 400-watt interior heat source and forcedair circulation generated by a 100 -watt interior fan blowing directly on the bottom polar steel plate.

1. After thermal equilibrium across the wall of the acrylic plastic capsule was established, the temperature measured on the interior surface of the wall was found to be approximately 3 to $4^{\circ} \mathrm{F}$ higher than the temperature of the water wetting the exterior of the hull.

2. After establishment of thermal equilibrium, the temperature of the air atmosphere contained within the hull was 12 to $12^{\circ} \mathrm{F}$ higher than the ambient temperature of the stagnant water wetting the exterior of the hull (Figure 120).

Tensile strength of joints in the 2-year-old 66-inch acrylic plastic capsule was established at the completion of the pressure test program by cutting tensile specimens from the fragments of the imploded capsule. Subsequently these bond test specimens were subjected to uniaxial tensile loading to determine their ultimate tensile strength after exposure to atmospheric environment for 2 years alternating with hydrospace environment while undergoing compressive fatigue testing in the vessel. The tensile strength of the joints bonded with PS- 18 adhesive was found to be 9,220 psi maximum, $5,680 \mathrm{psi}$ minimum, and $7,350 \mathrm{psi}$ average. When these strength values are compared with the 8,280-psi maximum, 5,150-psi minimum, and 6,700-psi average strength value of PS-18 adhesive (Table 3 ) measured immediately after casting of a 0.125 -inch-thick joint in 2.5 -inch-thick acrylic plastic, the following is evident:

1. A 2-year exposure to atmospheric environment alternating with hydrospace environment in the 0 -to-2,400-foot-depth range did not significantly alter the original strength of the joints.

2. The 107 pressure cycles in which the typical compressive stress in the joint varied from a minimum of about 600 psi to 11,000 psi had also no significant effect on the tensile strength of the joints bonded with PS-18 adhesive. 


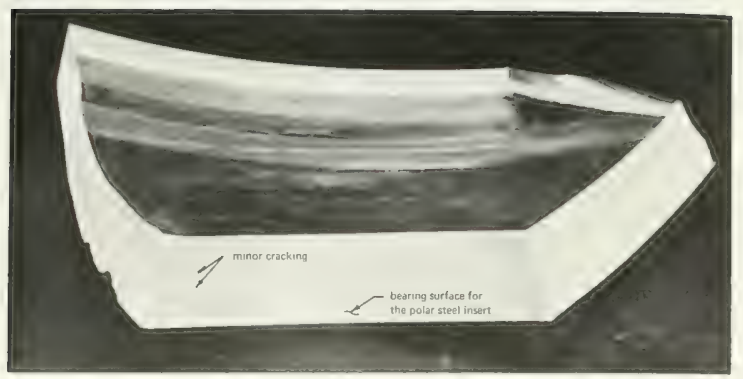

Figure 119a. Fragment from the top polar acrylic plastic pentagon. Note that the beveled bearing surface shows only minor cracking at the location that corresponds to the $\mathrm{O}$-ring groove on the

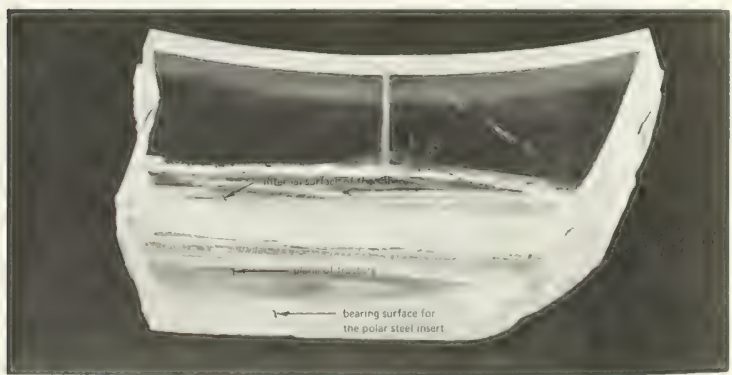

Figure $119 \mathrm{~b}$. Fragment from the bottom polar acrylic plastic pentagon. Note that serious spalling has taken place on the beveled bearing surface initiated at the location that corresponds to the $\mathrm{O}$-ring groove on the bottom steel plate. Mechanism of failure is shown in Figure $119 \mathrm{c}$ at operational depth, 600 feet

at 1.200 reet

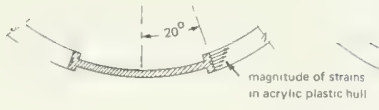

All deformations are of elastic nature

$$
\text { cegins to change }
$$

Intiation of vielding

at 4,000 feet

after fallure

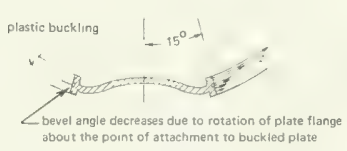

Plastic buckling just

orior to sohere implosion

Polar pentagon and tottom

plate after implosion

Figure $119 \mathrm{c}$. Mechanism of failure by spalling in the bottom polar acrylic plastıc pentagon reconstructed from observation of deformation in the bottom steel plate and fracture in the bottom polar acrylic plastic pentagon 



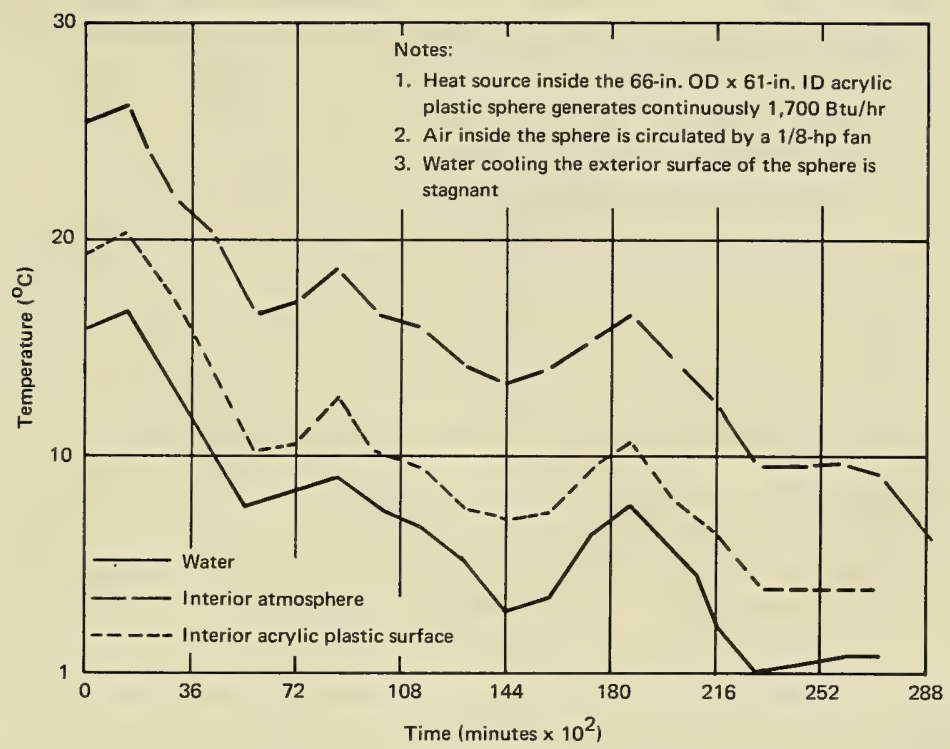

Figure 120. Influence of ambient water temperature on the temperature of the atmosphere contained within the 66-inch-diameter capsule in the presence of an internal heat source.

\section{SUMMARY OF FINDINGS}

1. The mechanical and physical properties of commercially available grade $G$ Plexiglas acrylic plastic plates procured according to NCEL specifications are reproducible within $\pm 10 \%$ from one plate to another in the whole 0.250 -to4.0-inch-thickness range, and the average of these properties matches the typical property values supplied by the manufacturer.

2. Fabrication of acrylic plastic spherical shells from flat cast plates that (a) have been thermoformed in a female vacuum-assisted mold, (b) machined into spherical pentagons, and (c) bonded together with PS-18 cement results in pressure hulls with dimensional deviations of approximately $\pm 6 \%$ in thickness and $\pm 0.5 \%$ in sphericity. 
3. The good reproducibility of material properties and capsule dimensions from one capsule to another as well as from small-scale models to large-scale capsules results in reliable structural performance, which varies for individual capsules less than $\pm 10 \%$ from the average collapse pressure of the group.

4. The 66-inch prototype capsule for incorporation into the NEMO system met the initial requirements for (1) 1,000 dives to design depth of 1,000 feet and (2) implosion under uncontrolled dive conditions only at depth in excess of 3,000 feet.

5. Both the design and the fabrication method developed for the 66-inch large-scale capsule prototype are applicable directly to the 120-inch-diameter capsule concept, the ultimate goal of the acrylic plastic capsule program.

6. Although the chosen polar plate dimensions appear to be adequate for the 1,000 -foot design depth when the plate is fabricated from type 316 stainless steel, the safety margin of the metal plates would be substantially increased if they were fabricated instead from Inconel 625 or cadmium plated SAE 4130 , both with a minimum yield point of 60,000 psi.

7. The use of models for validation of spherical capsule designs in acrylic plastic is acceptable; there appears to be no mass scaling factor associated with the scaling up of models to full-scale operational capsules.

\section{CONCLUSION}

The spherical hull concept of acrylic plastic developed in the NEMO program provides an economical, reliable, and safe pressure resistant capsule with panoramic visibility for continental shelf depths.

\section{RECOMMENDATIONS}

These recommendations are based on a conservative engineering evaluation of existing data.

1. On the basis of the experimental and analytical data developed in the 66-inch-OD $\times 61$-inch-ID acrylic plastic capsule program the NEMO capsule merits certification to 600 -foot depth. Brief proof-test dives are not to exceed 1,200-foot depth.

2. The crack-free fatigue life of the capsule for dives to 600 -foot depth shown in Figure 121 is recommended as a very safe fatigue rating for manned service. 


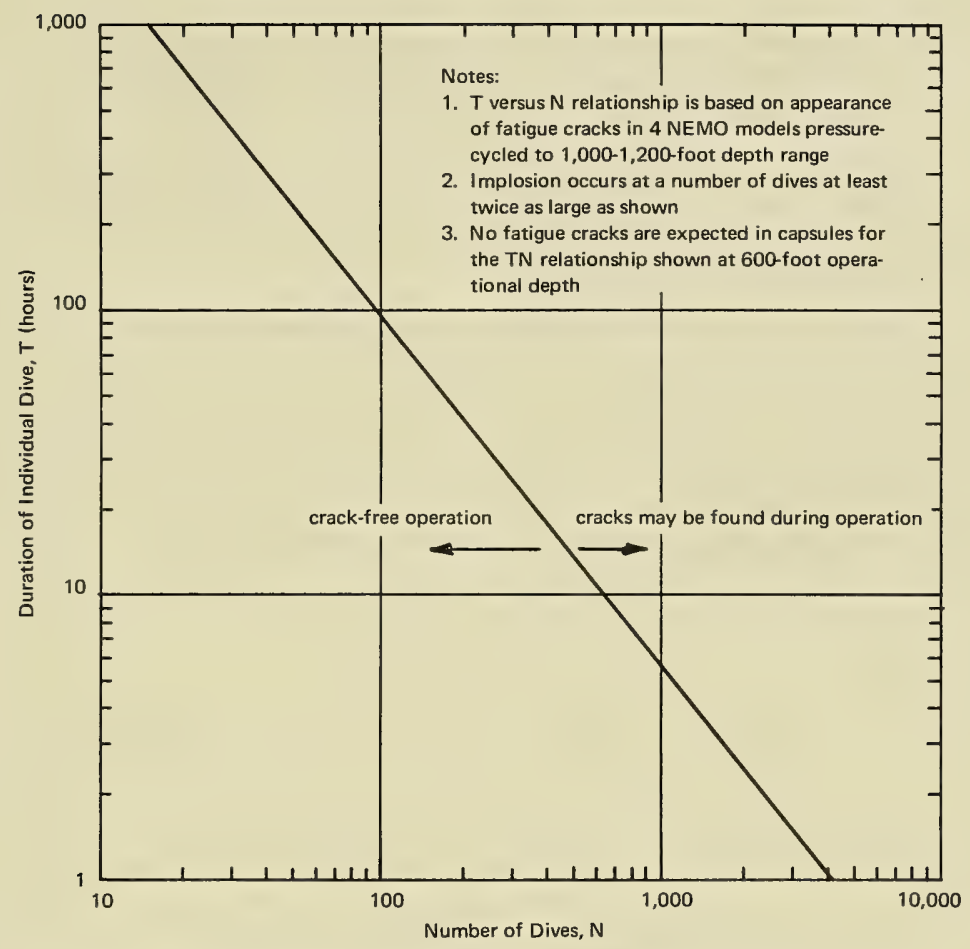

Figure 121. Crack-free fatigue life of 66-inch-OD NEMO capsule at 600-foot depth.

3. The fatigue life depends not only on the number of dives, but also on their maximum depth and duration. When the fatigue value of individual dive is calculated after each dive, both the duration and the maximum depth reached must be noted. The fatigue value of a dive to a lesser depth than 600 feet is calculated on the conservative linear basis (e.g., a dive to 300 feet of 1 -hour duration is considered as $1 / 2$ of a dive to 600 feet of 1 -hour duration). When the fatigue values for dives of different durations and depths are added up, the procedure is to consider each dive as a fraction of the available fatigue life at 600 feet and specified dive duration. Thus, the fatigue values of a 1 -hour dive to 300 feet, a 6 -hour dive to 400 feet, and a 100 -hour dive to 600 feet are added up as: 


$$
\sum=\frac{1}{4,000}\left(\frac{300}{600}\right)+\frac{1}{1,000}\left(\frac{400}{600}\right)+\frac{1}{100}\left(\frac{600}{600}\right)
$$

to result in a cumulative fatigue value of 0.01079 . So long as the summation of all dive fatigues is $\Sigma<1.000$ the hull is considered safe. When the sum of the dive fatigue values reaches 1.000 the hull will be considered to have no further fatigue life and must be replaced.

4. The fatigue curve of Figure 121 is very conservative as it is based on the appearance of cracks under cyclic proof-test depth loadings to 1,140 feet while the manned operational dives will have a maximum depth limit of only 600 feet. Furthermore, since it is known from the cycling tests conducted on models that extensive fracturing of the acrylic/metal plate interface takes place prior to catastrophic fatigue failure, periodic visual inspection of the hull during its service life will prevent premature fatigue failure.

5. These recommendations also apply to other acrylic plastic capsules that will be fabricated in the future for other systems besides NEMO, provided that:

a. The capsule design is identical to the one developed in this program.

b. Acrylic plastic material has the properties specified in Table 1.

c. Adhesive utilized has a minimum tensile strength of 4,500 psi and service life comparable to PS-18 adhesive in marine environment.

d. Thickness of material in the finished capsule is $2.285<\mathrm{t}<2.625$ inches.

e. The diameter of the capsule is $65.875<D<66.125$ inches.

f. The external radius of the capsule is $32.875<R<33.125$ inches.

\section{ACKNOWLEDGMENTS}

Many engineers and scientists have contributed to the development of the NEMO concept and the successful completion of the acrylic plastic capsule structural validation program. Messrs. R. G. McCarty, ${ }^{*}$ J. G. Moldenhauer, ${ }^{*}$ initiated the NEMO concept, while Messrs. K. Tsuji* and D. T. Stowell* developed it sufficiently to show its feasibility. The pentagonal acrylic plastic

* Naval Missile Center, Point Mugu, California. 
capsule concept was conceived and engineered by Dr. J. D. Stachiw* and designed by Messrs. W. A. Winner** and R. T. Rains. ${ }^{* *}$ The successful fabrication of the capsule is the result of close supervision of, and assistance to, shop personnel by Messrs. R. B. Gunyan, ${ }^{* *}$ D. O'Connor, ${ }^{* *}$ and Jack Marble.**

* Naval Civil Engineering Laboratory, Port Hueneme, California.

** Pacific Missile Range, Point Mugu, California. 


\section{DETAILED DIMENSIONS OF 66-INCH-DIAMETER ACRYLIC PLASTIC HULL FOR NEMO SYSTEM}

Although the description of the 66-inch acrylic plastic hull in the main body of the report is adequate for discussion of the acrylic plastic hull design and its performance under hydrostatic pressure, it is not detailed enough for the personnel involved in certifying the seaworthiness of the acrylic plastic hull. The Certification Board requires not only a detailed description of the plans from which the hull was built, but also how well the finished product conformed to these plans. Only after the relationship between the dimensions shown on plans and the dimensions of the finished product is well understood can the dimensional deviations of future operational hulls be considered as acceptable or unacceptable. This statement is based on the fact that the prototype 66-inch hull that successfully withstood the many hydrostatic overloads to which it was subjected during the testing program was not perfect, but deviated from the specified nominal dimensions in a certain manner. If future hulls built for incorporation into manned submersibles show only as much (or less) dimensional and structural deviation from fabrication plans and specifications as exhibited by the prototype, they will be acceptable. Their performance should then be equivalent or superior to the one shown by the prototype 66-inch acrylic plastic hull discussed in this report.

For this reason the figures and tables comprising Appendix $A$ are grouped to show in detail not only the drawings used in the fabrication, but also the dimensional measurements performed on the hull structure during and after the fabrication. Thus Figures $A-1$ to $A-10$ show the fabrication plans, while Figures $A-11$ to $A-13$ show the quality control measurements performed on the hull during fabrication. 


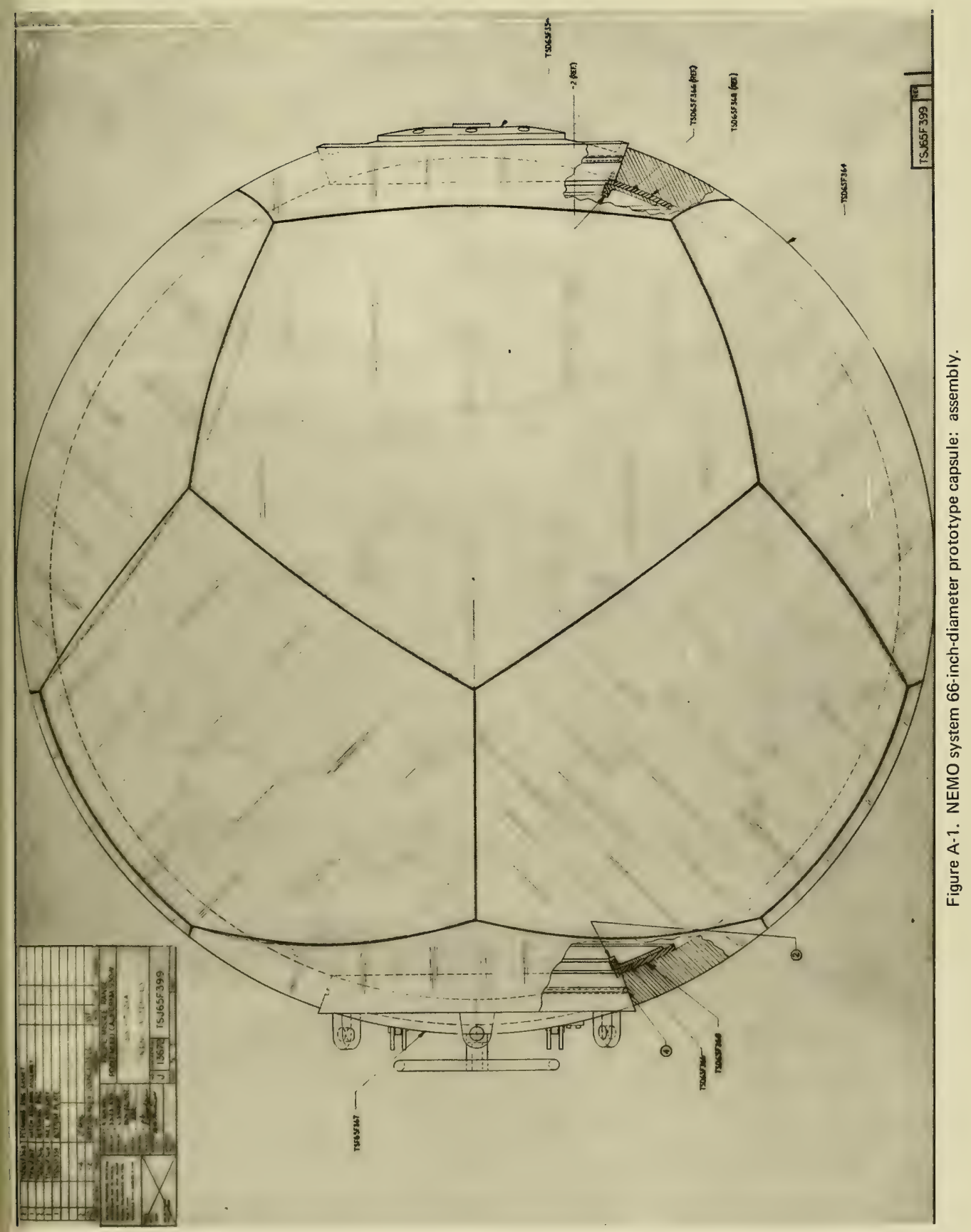




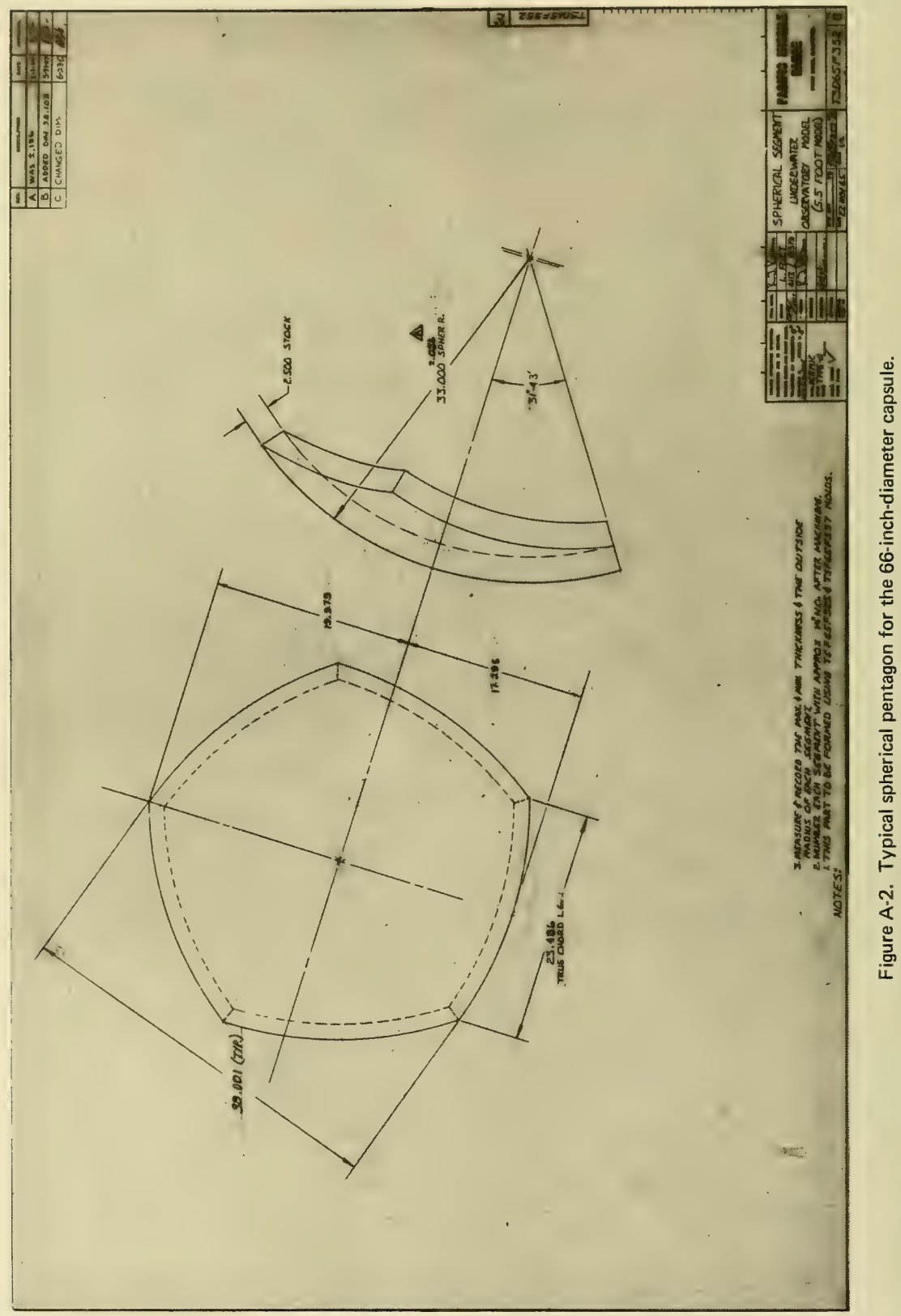




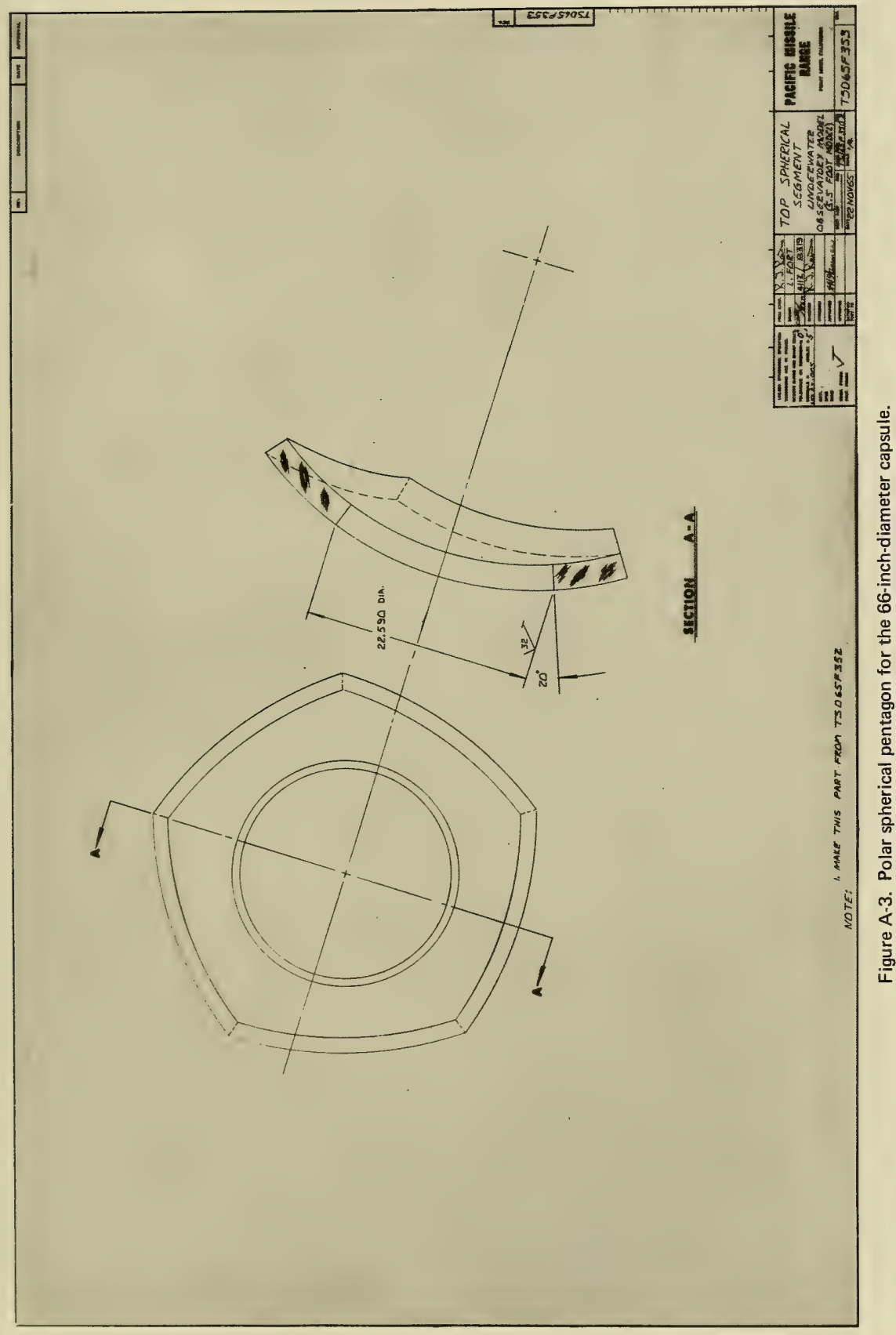




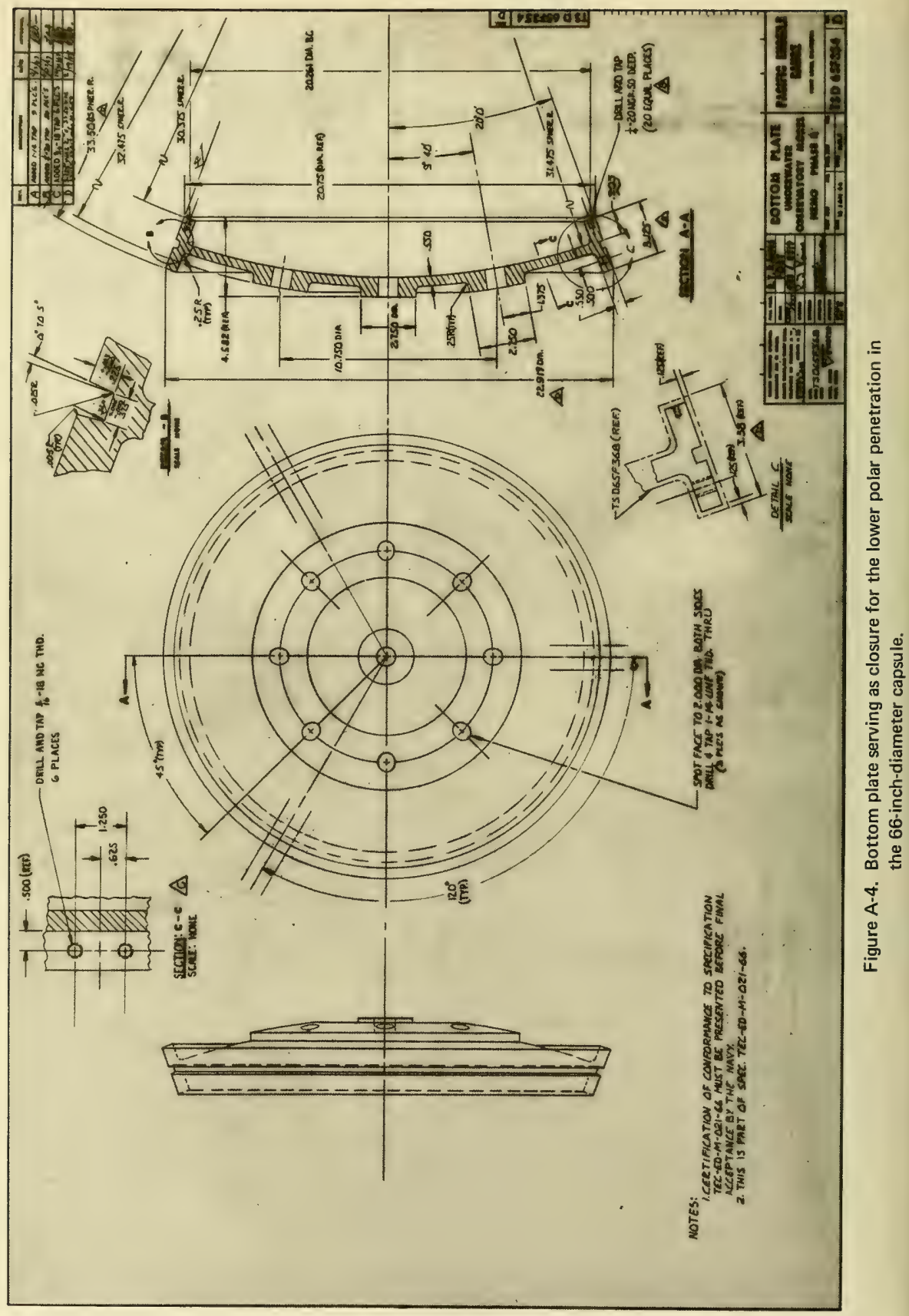




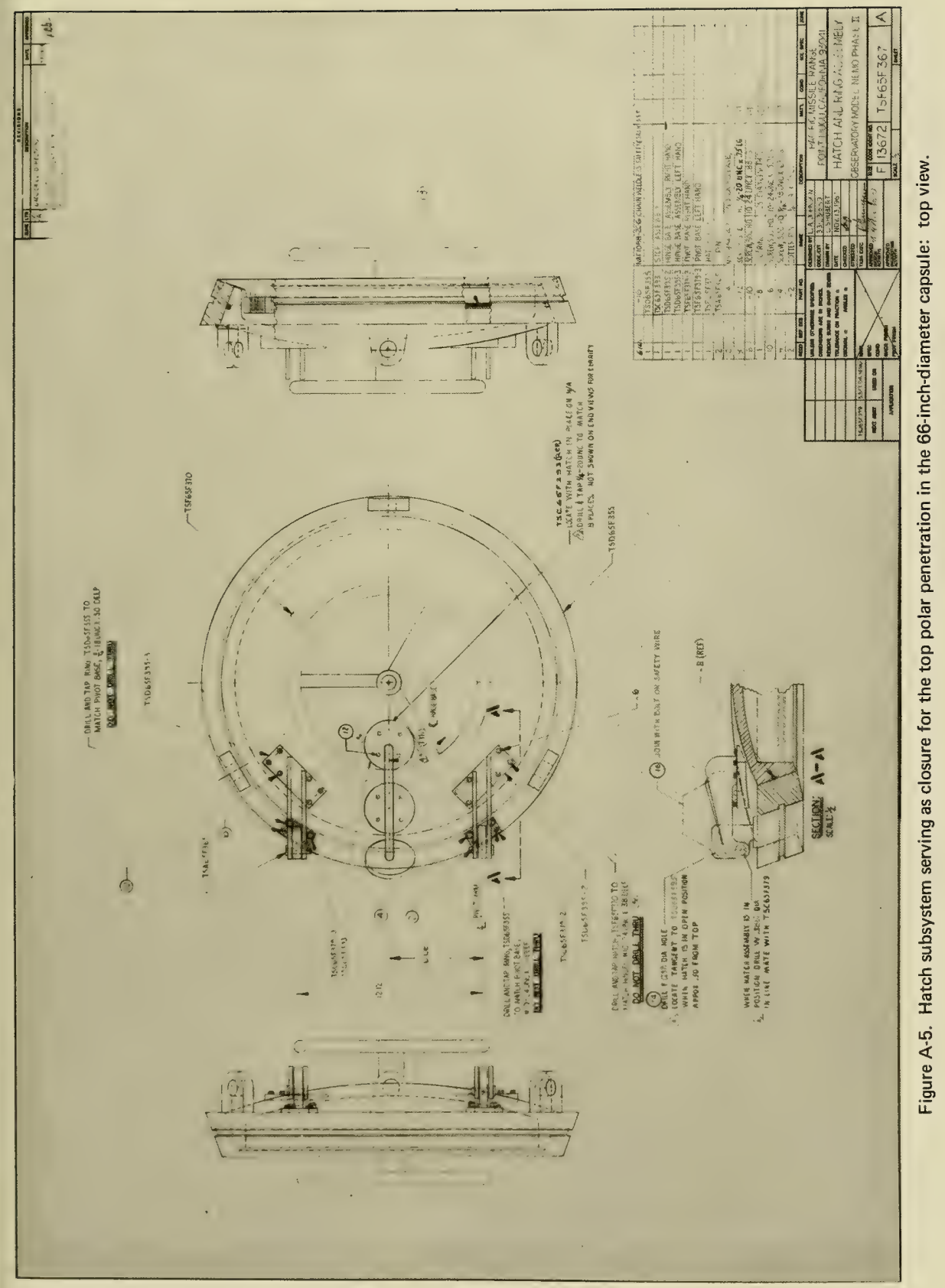




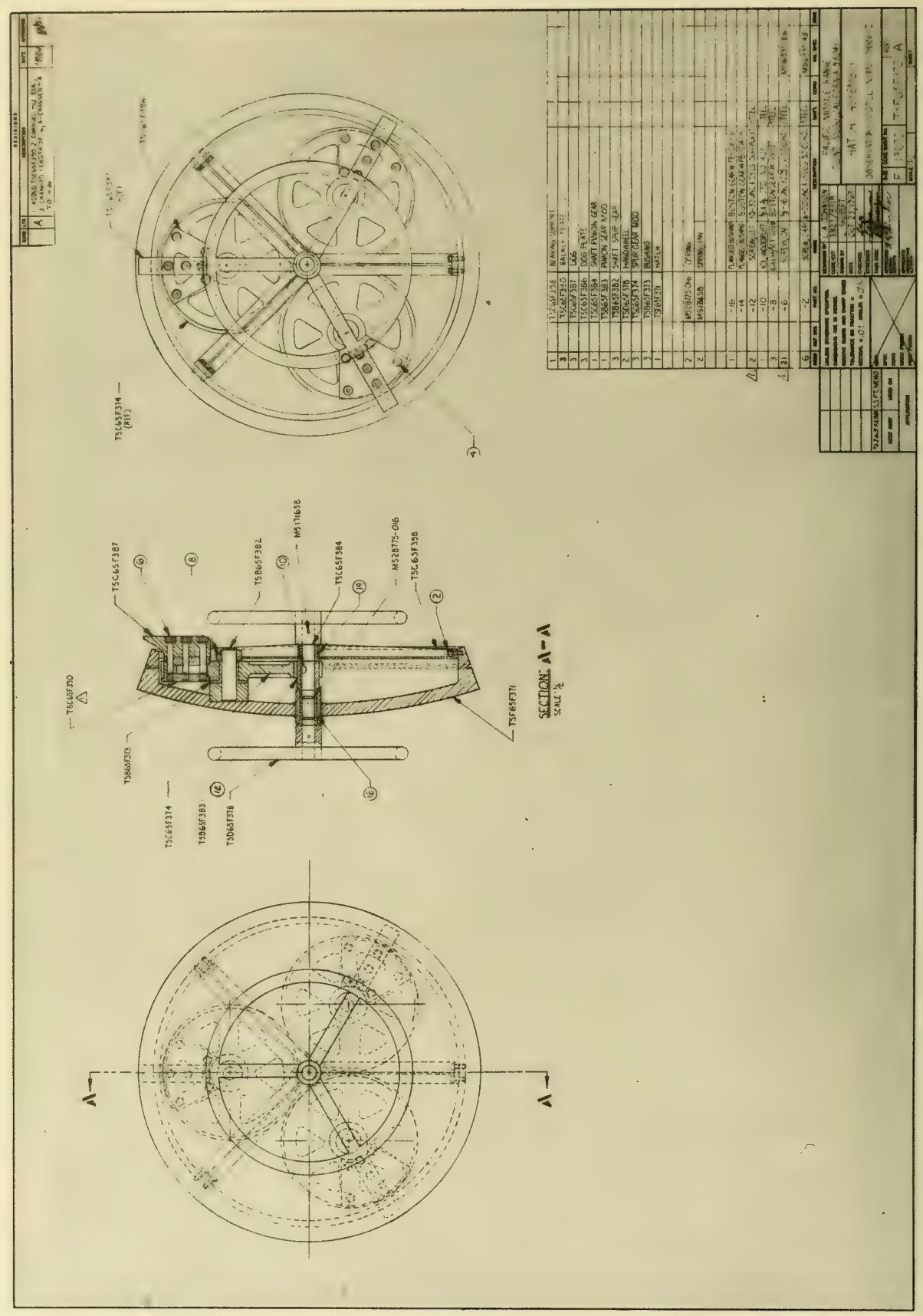

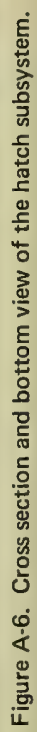




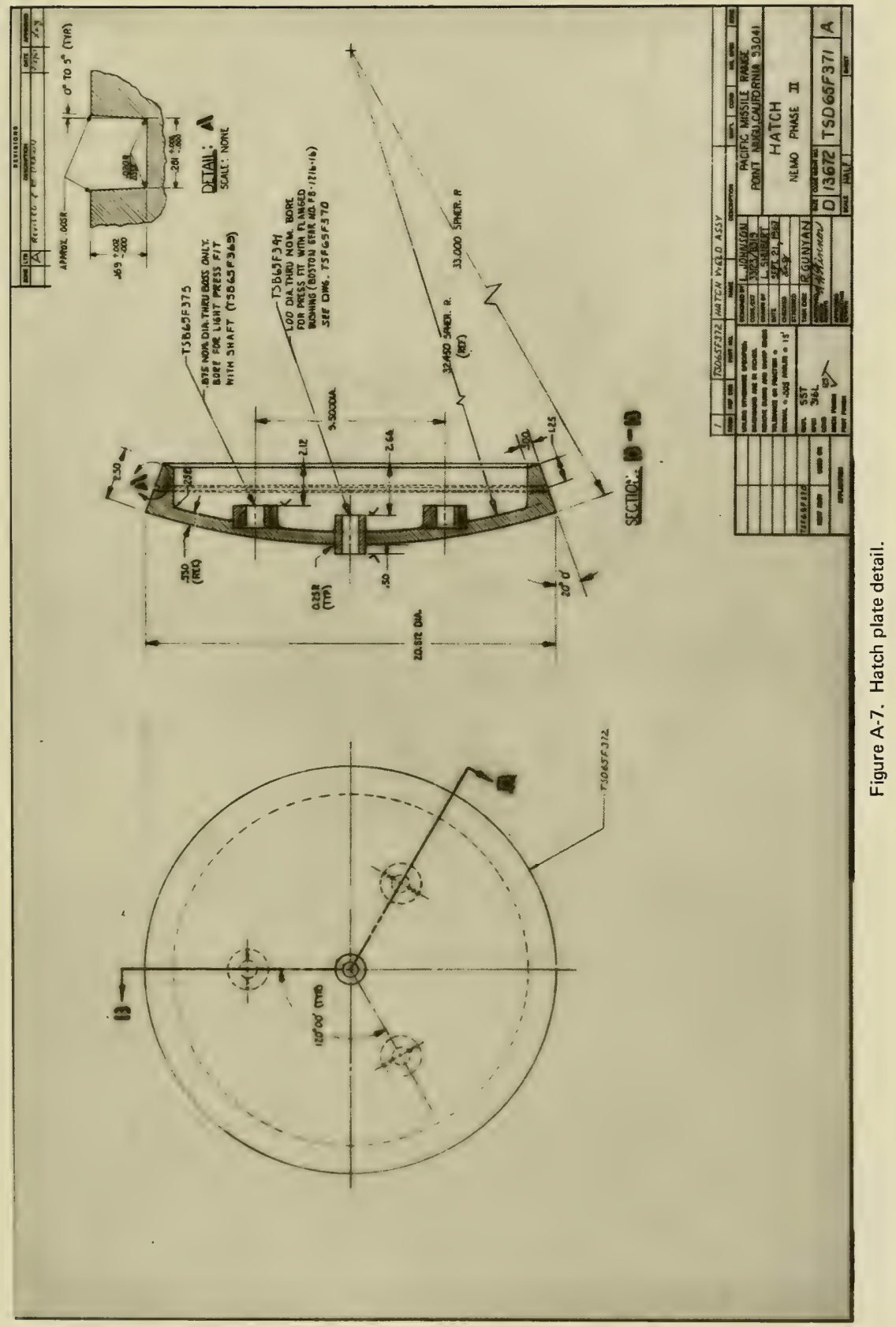




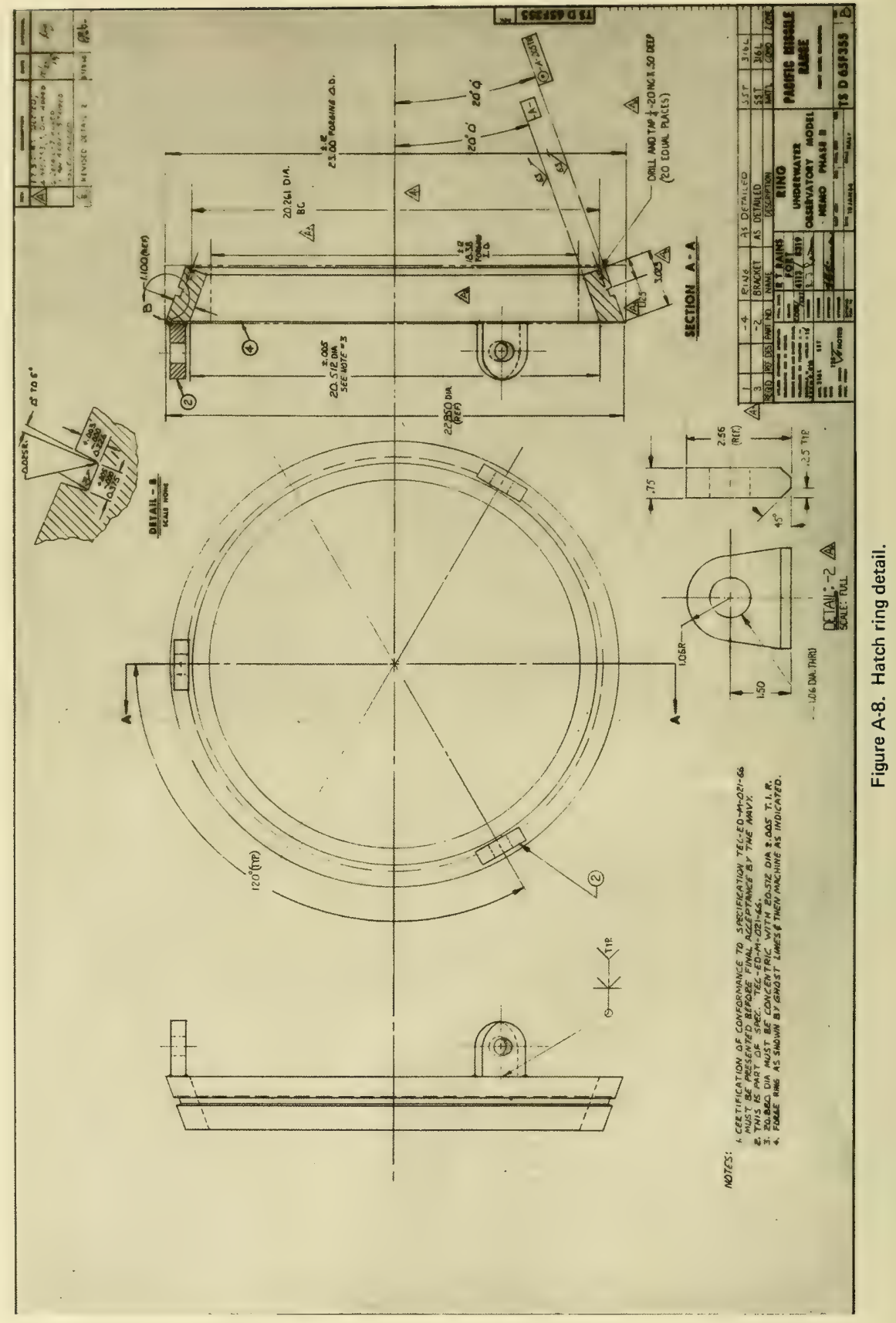




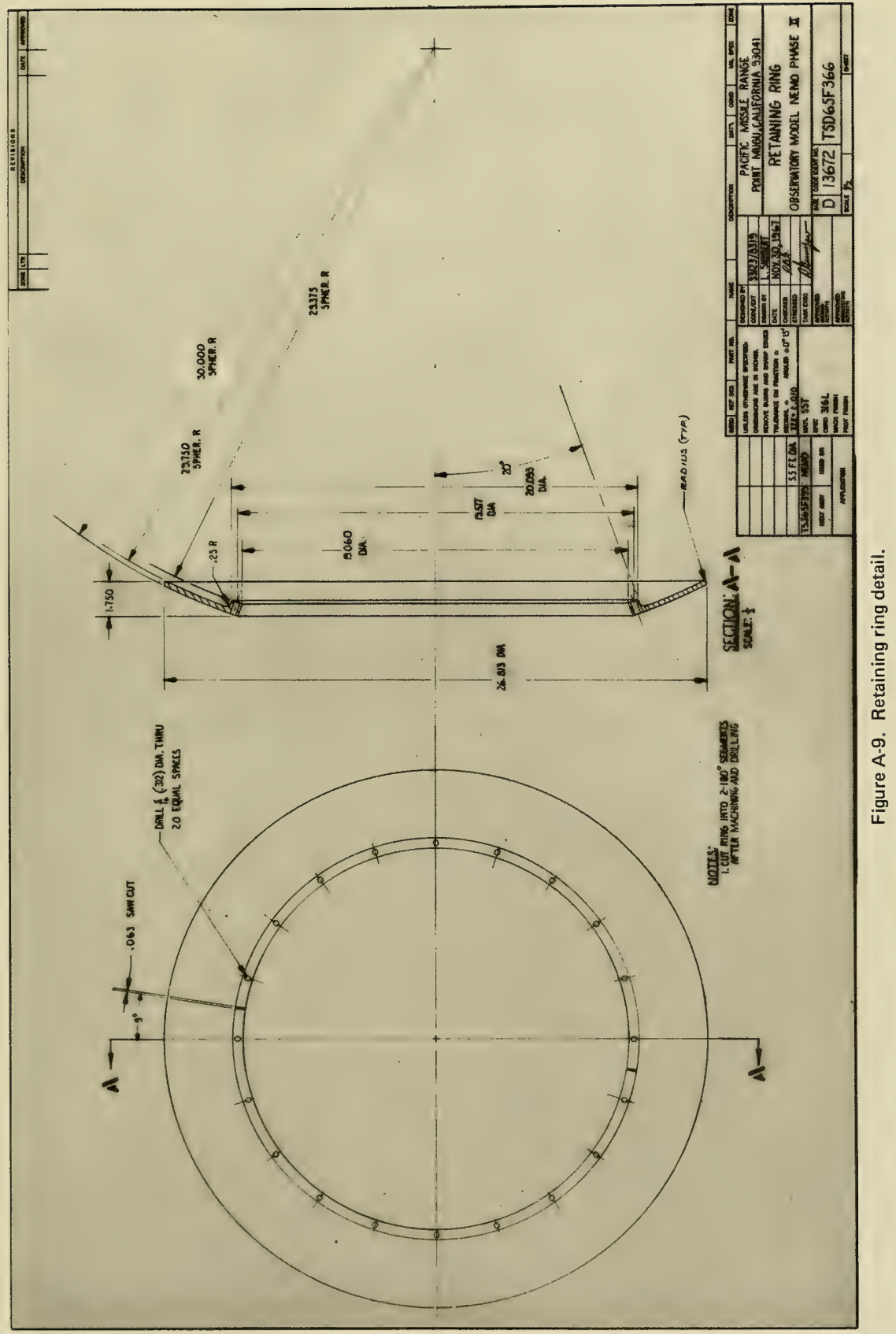




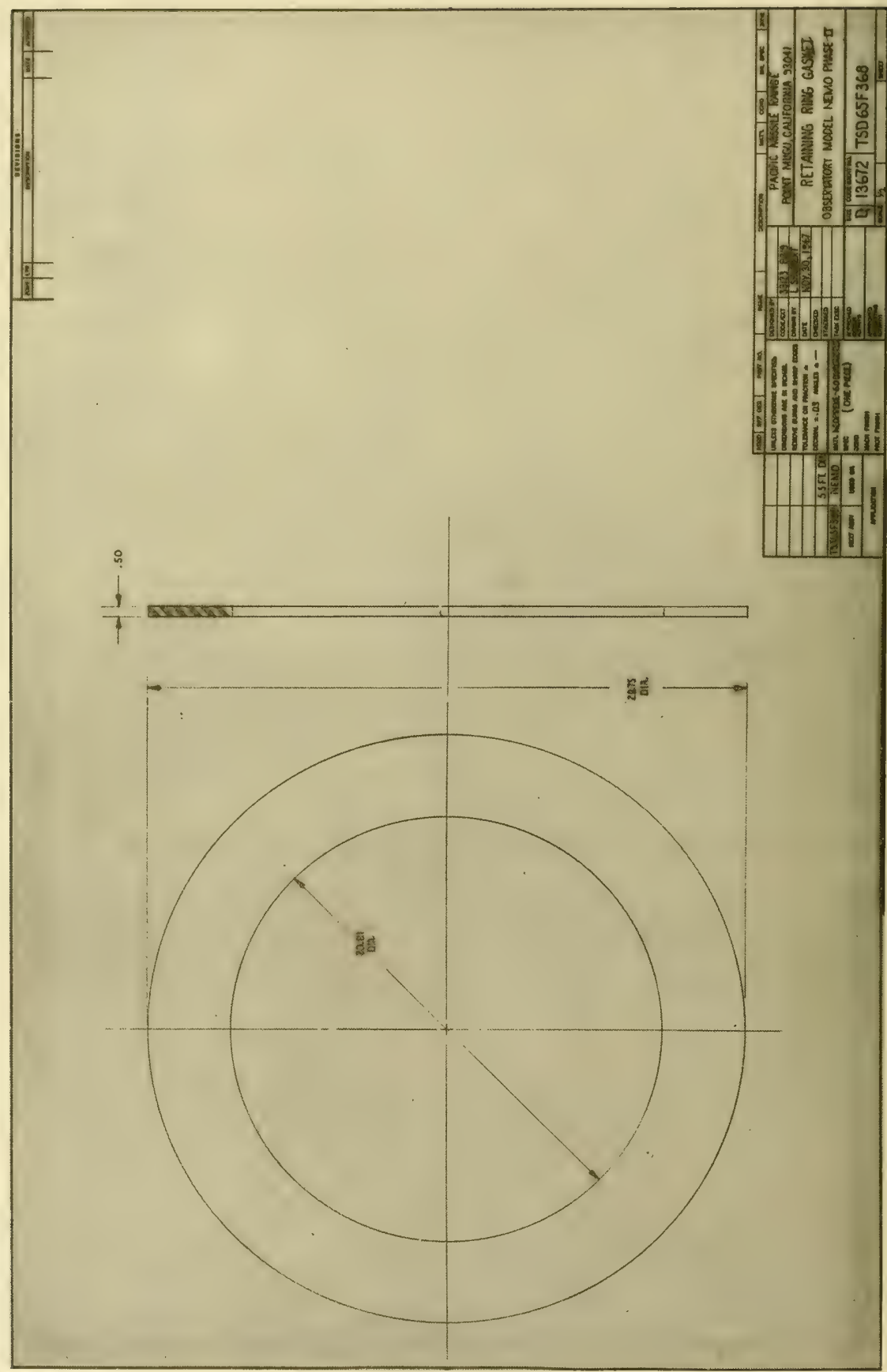

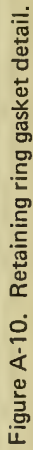


I. $10 \mathrm{R}^{\circ}$

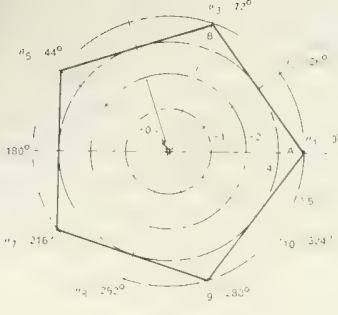

$T_{x \in \text { s I Pents, }}$

oangle

$00 \% \quad 431^{\circ} \mathrm{C}$

$D_{1}=10^{\circ} 30^{\prime} \quad 05=37^{\circ} 10$

: . 15

Notes

F, nts $A$, od $B$ where must ted to give grentalian

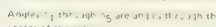

anceso or the somen

Angles $\theta_{1}$ through $\theta_{10}$ are measured from the

center of the pentagon

4 Pentayons are for the prolotyde 56 -inch NEMO tapsule

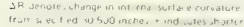
radrus while - indicates longer radius.

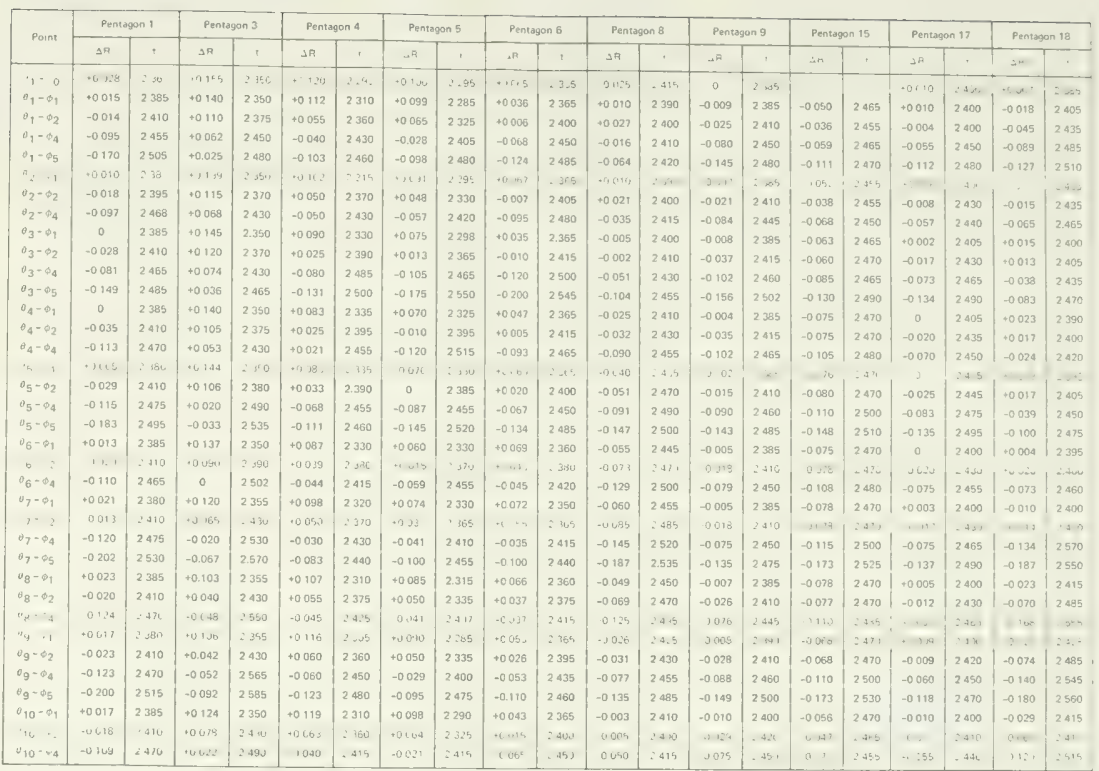

(d) Pentagons without penetrations.

Continued

Figure A.11. Quality control measurements; deviations trom nominal 33000 -1nch radus and 2 500-mch wall thickness in completed spherical pentagons pror to assembly into a sphere 


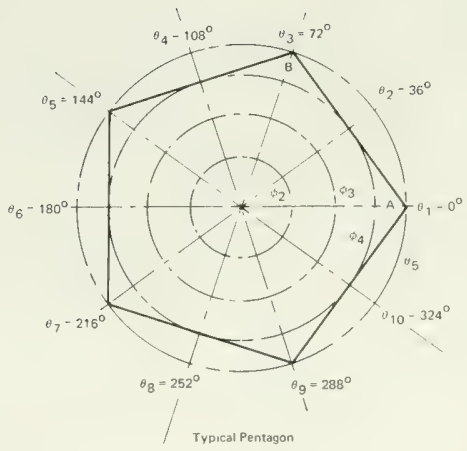

\begin{tabular}{|c|c|c|c|c|}
\hline \multirow{2}{*}{ Point } & \multicolumn{2}{|c|}{ Pentagon 2} & \multicolumn{2}{|c|}{ Pentagon 7} \\
\hline & $\Delta R$ & t & $\Delta R$ & t \\
\hline$\theta_{1}-\theta_{2}$ & to 105 & 2395 & +0041 & 2.460 \\
\hline$\theta_{1}-\phi_{3}$ & +0043 & 2455 & +0017 & 2485 \\
\hline$\theta_{1}-\theta_{4}$ & .0013 & 2510 & -0022 & 2520 \\
\hline$\theta_{1}-\phi_{5}$ & -0085 & 2540 & -0075 & 2540 \\
\hline$\theta_{2}-\theta_{2}$ & +0105 & 2395 & +0030 & 2465 \\
\hline$\theta_{2}-\theta_{3}$ & .0053 & 2455 & +0006 & 2490 \\
\hline$\theta_{2}-\phi_{4}$ & 0 & 2480 & -0035 & 2520 \\
\hline$\theta_{3}-\theta_{2}$ & +0110 & 2395 & +0028 & 2460 \\
\hline$\theta_{3}-\theta_{3}$ & +0061 & 2455 & 0 & 2490 \\
\hline$\theta_{3}-\phi_{4}$ & +0007 & 2480 & -0042 & 2520 \\
\hline$\theta_{3}-\theta_{5}$ & -0065 & 2530 & -0107 & 2550 \\
\hline$\theta_{4}-\theta_{2}$ & +0100 & 2395 & +0039 & 2465 \\
\hline$\theta_{4}-\phi_{3}$ & +0053 & 2445 & +0021 & 2480 \\
\hline$\theta_{4}-\phi_{4}$ & -0005 & 2480 & -0004 & 2505 \\
\hline$\theta_{5}-\theta_{2}$ & +0100 & 2395 & -0047 & 2440 \\
\hline $0_{5}-2_{3}$ & +0061 & 2445 & +0032 & 2460 \\
\hline$\theta_{5}-\theta_{4}$ & +0015 & 2465 & .0002 & 2480 \\
\hline$\theta_{5}=\phi_{5}$ & -0045 & 2500 & +0045 & 2505 \\
\hline$\theta_{6}=o_{2}$ & +0102 & 2395 & .0083 & 2420 \\
\hline${ }^{\theta} 6-\phi_{3}$ & +0040 & 2445 & +0069 & 2440 \\
\hline$\theta_{6}-\nabla_{4}$ & +0005 & 2470 & +0042 & 2460 \\
\hline$\theta_{7} \quad \phi_{2}$ & +0087 & 2395 & to 105 & 2410 \\
\hline$\theta_{7} \cdot \phi_{3}$ & +0051 & 2445 & +0095 & 2420 \\
\hline$\theta_{7}-s_{4}$ & +0003 & 2410 & +0060 & 2440 \\
\hline$\theta_{7}-\theta_{5}$ & -0055 & 2505 & +0003 & 2460 \\
\hline 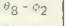 & .0090 & 2400 & +0.102 & 2400 \\
\hline$\theta_{8}=\theta_{3}$ & $+0,060$ & 2450 & +0093 & 2413 \\
\hline$\theta_{8}-\theta_{4}$ & +0037 & 2460 & +0052 & 2425 \\
\hline$\theta_{9}-\theta_{2}$ & +0095 & 2405 & +0095 & 2420 \\
\hline$\theta_{9} \cdot \phi_{3}$ & +0081 & 2445 & +0072 & 2445 \\
\hline$\theta_{9}-\theta_{4}$ & +0048 & 2455 & +0040 & 2465 \\
\hline$\theta_{9}-\omega_{5}$ & $-0,002$ & 2460 & -0010 & 2475 \\
\hline$a_{10}-\psi_{2}$ & +0095 & 2400 & .0057 & 2450 \\
\hline$\theta_{10}-\theta_{3}$ & .0050 & 2455 & +0 036 & 2475 \\
\hline $0.10-0_{4}$ & -0005 & 2475 & -0006 & 2497 \\
\hline
\end{tabular}

Q angle:

$\begin{array}{ll}\phi_{2}=21^{\circ} 0^{\prime} & \phi_{4}=31^{\circ} 30^{\prime} \\ \phi_{3}=26^{\circ} 15^{\prime} & \phi_{5}=37^{\circ} 10^{\prime}\end{array}$

Notes

1. Points $A$ and $B$ were marked to give orientation.

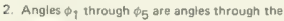
center of the sphere.

3. Angles $\theta_{1}$ through $\theta_{10}$ are measured from the center of the pentagon.

4. Pentagons are for the prototype 66-inch NEMO capsule

5. $\triangle R$ denotes change in internal surface curvature from specified 30.500 inches; + indicates shorter radius, while - indicates longer radius.

(b) Pentagons with penetrations.

Figure A-11. Continued. 


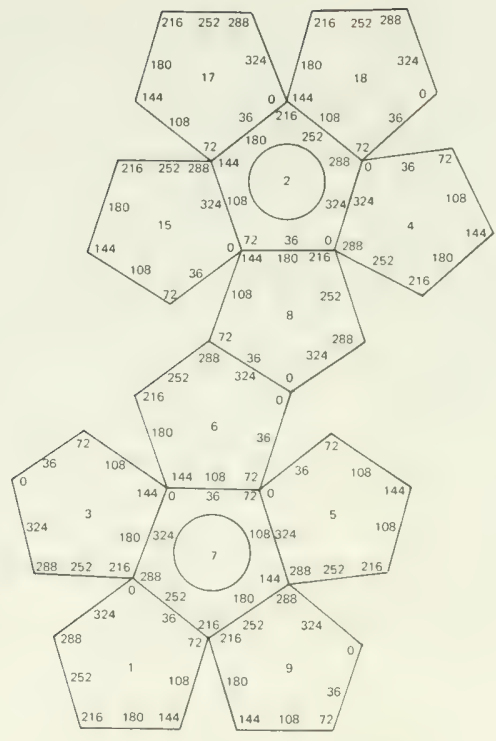

\begin{tabular}{|c|c|c|c|c|c|c|}
\hline From & To & $\begin{array}{l}\text { Diameter } \\
\text { (in) }\end{array}$ & Date & Tome & $\begin{array}{c}\text { Temperature } \\
\left({ }^{\circ} \mathrm{F}\right)\end{array}$ & Humidity \\
\hline 9 & 15 & 66125 & 1219.67 & 1420 & 74 & 47 \\
\hline 1 & 8 & 66130 & 121967 & 1430 & 74 & 47 \\
\hline 4 & 3 & 66084 & 12.19 .67 & 1440 & 74 & 47 \\
\hline 6 & 18 & 66135 & $12 \cdot 19 \cdot 67$ & 1450 & 74 & 47 \\
\hline 5 & 17 & 66158 & $12.19-67$ & 1500 & 74 & 47 \\
\hline 20 & 7.288 & 66055 & 12.19 .67 & 1530 & 74 & 47 \\
\hline 2.72 & 7.216 & 66032 & $12 \cdot 20-67$ & 0735 & 65 & 44 \\
\hline $2-144$ & $7-144$ & 66065 & $12 \cdot 20-67$ & 0745 & 65 & 44 \\
\hline 2.216 & 7.72 & 66070 & $32.20-67$ & 0750 & 66 & 44 \\
\hline 2.288 & $7-0$ & 66071 & $12-20-67$ & 0800 & 66 & 44 \\
\hline 40 & $3-144$ & 66060 & $12-20-67$ & 0800 & 66 & 44 \\
\hline 472 & $3-72$ & 66005 & $12 \cdot 20-67$ & 0800 & 66 & 44 \\
\hline 4144 & $3-0$ & 65920 & $12.20-67$ & 0805 & 66 & 44 \\
\hline 4216 & $3-288$ & 65975 & $12-20-67$ & 0810 & 66 & 44 \\
\hline 4288 & 3216 & 66035 & $12 \cdot 20-67$ & 0810 & 66 & 44 \\
\hline 8-70 & 1216 & 65945 & $12 \cdot 20-67$ & 0815 & 66 & 44 \\
\hline 8.72 & 1144 & 66000 & $12-20-67$ & 0815 & 67 & 44 \\
\hline 8.144 & 172 & 66030 & $12-20-67$ & 0820 & 67 & 44 \\
\hline 8.216 & 1.0 & 66010 & $12 \cdot 20-67$ & 0820 & 67 & 44 \\
\hline 8.288 & 1288 & 65938 & $12-20-67$ & 0825 & 67 & 44 \\
\hline $15-0$ & $9-216$ & 66015 & $12-20-67$ & 0830 & 68 & 44 \\
\hline 15.72 & 9144 & 65978 & $12-20-67$ & 0830 & 68 & 44 \\
\hline 15144 & 972 & 65975 & $12-20-67$ & 0830 & 68 & 44 \\
\hline 15.216 & $9-0$ & 66010 & $12.20-67$ & 0835 & 68 & 44 \\
\hline 15.288 & 9288 & 66050 & $12-20-67$ & 0835 & 69 & 44 \\
\hline 180 & 6.216 & 65992 & $12-20-67$ & 0840 & 58 & 44 \\
\hline 18.72 & $6-144$ & 66045 & $12-20-67$ & 0840 & 68 & 44 \\
\hline $18-144$ & 6.72 & 66050 & $12-20-67$ & 0840 & 68 & 44 \\
\hline 18.216 & $6-0$ & 65985 & 12.2067 & 0845 & 53 & 44 \\
\hline $18-288$ & 6.288 & 65965 & $12 \cdot 20-67$ & 0845 & 68 & 44 \\
\hline 80 & 1.216 & 65950 & $1 \cdot 3.68$ & 0945 & 64 & 40 \\
\hline 872 & 1.144 & 66075 & $1-3-68$ & 0950 & 64 & 40 \\
\hline 8.144 & 1.72 & 66075 & $1-3-68$ & 1000 & 64 & 40 \\
\hline 8.216 & 1.0 & 66080 & $1-3-68$ & 1000 & 64 & 40 \\
\hline 8.288 & 1288 & 66085 & $1-3.68$ & 1010 & 64 & 40 \\
\hline 80 & 1.216 & 65990 & $1-3-68$ & 1115 & 67 & 38 \\
\hline 872 & 1.144 & 66015 & $1-3-68$ & 1115 & 67 & 38 \\
\hline $8-144$ & 1.72 & 66010 & $1-3.68$ & 1125 & 67 & 38 \\
\hline 8216 & 1.0 & 66085 & 1.3 .68 & 1135 & 67 & 38 \\
\hline 8. 288 & 1.288 & 66085 & $1.3-68$ & 1145 & 67 & 38 \\
\hline 8.0 & 1.216 & 65950 & $1-3.68$ & 1505 & 12 & 36 \\
\hline 8.72 & 1144 & 66015 & $1 \cdot 3-68$ & 1510 & 72 & 36 \\
\hline 8. 144 & 1.72 & 66060 & $1-3-68$ & 1515 & 72 & 36 \\
\hline 8216 & 1.0 & 66010 & $1-3-68$ & 1520 & 72 & 36 \\
\hline 8. 288 & 1.288 & 66085 & $1-3 \cdot 68$ & 1520 & 72 & 36 \\
\hline
\end{tabular}

Layout of Prototype 66-Inch Capsule For NEMO System

\section{Nore}

1. Diameter measurements taken 0,50 inch from pentagon's corner as shown on plan layout of NEMO.

2. Measurament at 9.15 , etc. is center of pentagon.

Figure A-12. Quality control measurements; deviations from nominal 66.000 -inch diameter after assembly and bonding of pentagons into a 66 -inch-diameter capsule. 


\begin{tabular}{|c|c|c|c|c|c|c|c|c|c|c|c|c|c|c|c|c|c|c|c|c|c|c|c|c|c|}
\hline $\begin{array}{l}\text { Intersection } \\
\text { of Pentagons }\end{array}$ & Corner & 1 & 11 & 111 & IV & v & $v_{1}$ & Center & 1 & 11 & III & IV & v & $v_{1}$ & Corner & 1 & " & III & IV & $v$ & $v_{1}$ & Date & Time & $\begin{array}{c}\text { Temperature } \\
{ }^{\circ} \mathrm{F} \mid\end{array}$ & Humidity \\
\hline $2-4$ & $288-0$ & - & 0.012 & 0.045 & 0.045 & 0.015 & 0.000 & 324324 & - & - & 0.014 & 0.025 & 0.006 & 0.005 & 0.288 & - & 0.004 & 0.045 & 0,050 & 0015 & 0000 & $12 \cdot 16-67$ & 1250 & 74 & 40 \\
\hline 28 & 0.216 & - & 0035 & 0.062 & 0.062 & 0.039 & 0.009 & $36-180$ & - & - & 0.045 & 0.045 & 0018 & 0.010 & 72.144 & - & 0.041 & 0.070 & 0.070 & 0041 & 0013 & $12-16-67$ & 1300 & 74 & 40 \\
\hline 2.15 & 72.0 & - & 0.032 & 0.070 & 0.072 & 0.036 & 0.014 & $108-324$ & - & - & 0.042 & 0.042 & 0.016 & 0.011 & $144-288$ & - & 0.031 & 0.066 & 0.066 & 0035 & 0014 & $12-16.67$ & 1345 & 74 & 40 \\
\hline 2.17 & $144-72$ & - & 0.039 & 0.059 & 0.061 & 0.023 & 0,005 & $180-36$ & - & - & 0.036 & 0.045 & 0.036 & 0.016 & $216-0$ & - & 0017 & 0.055 & 0.060 & 0031 & 0010 & $12 \cdot 16-67$ & 1400 & 74 & 41 \\
\hline 2.18 & 216.144 & - & 0.001 & 0.035 & 0.039 & 0.020 & 0.007 & $252-108$ & - & - & 0.003 & 0.012 & 0.008 & 0.009 & 288.72 & - & 0000 & 0016 & 0033 & 0017 & 0014 & $12 \cdot 16-67$ & 1430 & 74 & 41 \\
\hline 418 & 0.72 & 0.005 & 0.013 & 0.041 & 0.039 & 0.019 & 0.014 & 36-36 & 0.013 & 0.000 & 0.019 & 0.036 & 0.023 & 0.010 & $72-0$ & 0.003 & 0.000 & 0.028 & 0031 & 0013 & 0009 & $12-16-67$ & 1500 & 74 & 41 \\
\hline 49 & $72-72$ & 0010 & 0.021 & 0.064 & 0.074 & 0045 & 0.025 & $108-36$ & 0.038 & 0.045 & 0.078 & 0.084 & 0.045 & 0.030 & 144.0 & 0.040 & 0.075 & 0.119 & 0.099 & 0038 & 0029 & 12.18 .67 & 1135 & 74 & 48 \\
\hline 45 & 144216 & 0.042 & 0.080 & 0.134 & 0.115 & 0.061 & 0.033 & $180-180$ & 0.040 & 0048 & 0.090 & 0.074 & 0.048 & 0.034 & $216-144$ & 0.016 & 0.035 & 0.070 & 0.074 & 0046 & 0026 & 12.18 .67 & 1235 & 75 & 49 \\
\hline 48 & $216-288$ & 0013 & 0.018 & 0.048 & 0.045 & 0006 & 0.002 & $252-252$ & 0.023 & 0020 & 0.043 & 0039 & 0.014 & 0.012 & 288.216 & 0.010 & 0027 & 0.660 & 0060 & 0029 & 0011 & $12-18.67$ & 0800 & 68 & 46 \\
\hline 8.15 & 144.0 & 0.020 & 0.042 & 0.075 & 0.090 & 0.045 & 0028 & $108-36$ & 0.026 & 0.037 & 0.074 & 0.077 & 0.045 & 0.031 & $72-72$ & 0013 & 0028 & 0076 & 0076 & 0037 & 0025 & $12-18-67$ & 0830 & 70 & 47 \\
\hline 8.6 & 0.0 & 0.036 & 0.068 & 0.120 & 0.118 & 0.055 & 0.024 & $36-324$ & 0.031 & 0.047 & 0.080 & 0080 & 0.044 & 0.028 & 72.288 & 0020 & 0.041 & 0.085 & 0092 & 0040 & 0022 & 12.18 .67 & 1300 & 75 & 48 \\
\hline 85 & 0.72 & 0.028 & 0057 & 0.112 & 0091 & 0.045 & 0.027 & $324-108$ & 0.026 & 0.031 & 0076 & 0074 & 0.036 & 0.030 & $288-144$ & 0010 & 0027 & 0.068 & 0094 & 0045 & 0034 & $12-18.67$ & 1336 & 76 & 47 \\
\hline $15-17$ & $288-72$ & 0.005 & 0.017 & 0.015 & 0.043 & 0006 & 0000 & $252-108$ & 0.008 & 0.008 & 0.31 & 0.026 & 0.008 & 0.009 & $216 \cdot 144$ & 0000 & 0.003 & 0035 & 0043 & 0005 & 0005 & $12.18-67$ & 1020 & 72 & 46 \\
\hline 15.3 & 144.72 & 0.004 & 0.028 & 0.064 & 0.055 & 0.036 & 0.028 & $180-36$ & 0.006 & 0.003 & 0.020 & 0075 & 0.050 & 0.036 & $216-0$ & 0.006 & 0002 & 0.030 & 0.081 & 0060 & 0.038 & $12.18-67$ & 1430 & 16 & 48 \\
\hline $15-6$ & 72.288 & 0.028 & 0.048 & 0.095 & 0.104 & 0.050 & 0.030 & $108-252$ & 0.040 & 0.057 & 0.092 & 0.091 & 0.048 & 0.035 & 144216 & 0.030 & 0.064 & 0.120 & 0.115 & 0060 & 0034 & $12 \cdot 18-67$ & 1510 & 76 & 48 \\
\hline 17.18 & 0.144 & 0009 & 0.035 & 0.076 & 0.062 & 0.026 & 0.014 & $324-180$ & 0.013 & 0.020 & 0.054 & 0047 & 0.026 & 0.026 & $288-216$ & 0.009 & 0.020 & 0053 & 0.050 & 0014 & 0018 & 12.18 .67 & 1050 & 73 & 48 \\
\hline $17-1$ & $216-288$ & 0032 & 0.062 & 0.110 & 0.110 & 0.069 & 0.041 & $252-252$ & 0.029 & 0050 & 0.080 & 0.102 & 0.045 & 0.036 & 288.216 & 0020 & 0060 & 0.085 & 0.100 & 0045 & 0025 & $12-19-67$ & 0745 & 63 & 46 \\
\hline 17.3 & $216-288$ & 0010 & 0036 & 0.080 & 0.090 & 0.065 & 0039 & $180-324$ & 0.010 & 0010 & 0.030 & 0.090 & 0.064 & 0.048 & 1440 & 0002 & 0002 & 0.028 & 0095 & 0068 & 0047 & 12.19 .67 & 0805 & 70 & 46 \\
\hline $18-1$ & $216-276$ & 0010 & 0.014 & 0057 & 0.075 & 0.034 & 0010 & 252.180 & 0.026 & 0.035 & 0.050 & 0.045 & 0.015 & 0.010 & $288 \cdot 144$ & 0024 & 0.042 & 0070 & 0.062 & 0016 & 0007 & $12-19 \cdot 67$ & 0835 & 70 & 46 \\
\hline 18.9 & 0.72 & 0.019 & 0.033 & 0.060 & 0.070 & 0.041 & 0.020 & 324.108 & 0.026 & 0.035 & 0.050 & 0.062 & 0.035 & 0.023 & $288-144$ & 0030 & 0047 & 0080 & 0.092 & 0047 & 0027 & $12 \cdot 19 \cdot 67$ & 0850 & 70 & 46 \\
\hline $9-1$ & $144-144$ & 0.006 & 0.030 & 0.069 & 0.048 & 0.011 & 0.003 & $180-108$ & 0020 & 0.022 & 0050 & 0.032 & 0.010 & 0.010 & 216.72 & 0000 & 0.012 & 0062 & 0062 & 0006 & 0000 & $12.19-67$ & 0940 & 72 & 46 \\
\hline 9.7 & $216-216$ & 0.010 & 0.020 & 0.045 & 0.050 & 0.025 & 0.012 & $252-180$ & 0.012 & 0.012 & 0.033 & 0034 & - & - & $288-144$ & 0006 & 0.020 & 0048 & 0033 & 0010 & - & 12.19 & 1000 & 72 & 46 \\
\hline 95 & 0.216 & 0.010 & 0.035 & 0.072 & 0.074 & 0.032 & 0.013 & $324-252$ & 0011 & 0022 & 0.050 & 0045 & 0.022 & 0.018 & $288-288$ & 0001 & 0012 & 0.050 & 0.032 & 0010 & 0002 & $12 \cdot 19 \cdot 67$ & 1020 & 72 & 46 \\
\hline $5-7$ & 288.144 & 0.002 & 0.010 & 0.033 & 0.030 & 0.002 & - & 324108 & 0.010 & $0.010^{2}$ & 0.025 & 0020 & - & - & 0.72 & 0.010 & 0.012 & 0.040 & 0.037 & 0000 & & $12-19.67$ & 1045 & 72 & 46 \\
\hline $5 \cdot 6$ & 0.72 & 0.003 & 0.013 & 0.041 & 0.055 & 0.014 & 0.002 & $36-36$ & 0.084 & 0.020 & 0.047 & 0.045 & 0.014 & 0.006 & 72.0 & 0.005 & 0.025 & 0.066 & 0.068 & 0029 & 0006 & $12 \cdot 19 \cdot 67$ & 1100 & 72 & 46 \\
\hline $6-7$ & 72.72 & 0.003 & 0.013 & 0.048 & 0.042 & 0.007 & - & 108.36 & 0.082 & 0.012 & 0.029 & 0.022 & - & - & 1440 & 0.003 & 0.012 & 0.044 & 0.035 & 0007 & - & $12 \cdot 19.67$ & 1125 & 73 & 46 \\
\hline 6.3 & $216-72$ & 0.000 & 0.008 & 0.046 & 0.034 & 0.022 & 0007 & $180-108$ & 0.004 & 0.002 & 0.016 & 0.026 & 0.021 & 0.020 & $216-72$ & 0000 & 0.005 & 0.027 & 0.022 & 0008 & 0009 & 67 & 1235 & 74 & 47 \\
\hline 1.7 & $72-216$ & 0.010 & 0.018 & 0.060 & 0.064 & 0.025 & - & $36-252$ & 0.015 & 0016 & 0.046 & 0048 & - & - & 0.288 & 0015 & 0.029 & 0076 & 0.060 & 0027 & - & $12-19.67$ & 1300 & 74 & 47 \\
\hline 37 & $216-288$ & 0.010 & 0.015 & 0.016 & 0.020 & 0.000 & - & $180-324$ & 0.010 & 0015 & 0016 & 0008 & - & - & 1440 & 0.009 & 0.015 & 0.015 & 0.017 & 0002 & & $12.19-67$ & 1315 & 74 & 47 \\
\hline 3.1 & 288.288 & 0.016 & 0.036 & 0.039 & 0.051 & 0.018 & 0.005 & $252-324$ & 0.017 & 0034 & 0042 & 0034 & 0.008 & 0.007 & 216.0 & 0.012 & 0.017 & 0.033 & 0.050 & 0015 & 0000 & 12.19 .67 & 1330 & 74 & 47 \\
\hline 91 & $144-144$ & 0.007 & 0.018 & 0.062 & 0.043 & 0.006 & 0.002 & 180.108 & 0.006 & 0.015 & 0.045 & 0.032 & 0.005 & 0.005 & $216-72$ & 0.000 & 0.004 & 0.035 & 0.040 & 0004 & 0002 & $1-3.68$ & 0920 & 64 & 42 \\
\hline $9-1$ & 144.144 & 0.007 & 0.018 & 0.064 & 0045 & 0.003 & 0.002 & 180.108 & 0009 & 0.014 & 0.043 & 0027 & 0.002 & 0003 & 216.72 & 0.002 & 0.010 & 0037 & 0.035 & 0006 & 0001 & 1.3 .68 & 1105 & 67 & 38 \\
\hline $9-1$ & 144144 & 0.007 & 0.017 & 0.069 & 0.045 & 0.005 & 0.002 & 180.108 & 0.014 & 0.018 & 0.050 & 0.032 & 0.006 & 0.009 & $216-72$ & 0.001 & 0.010 & 0.042 & 0.035 & 0006 & 0002 & $1-368$ & 1500 & 72 & 36 \\
\hline
\end{tabular}

Prorotype 66-inch Capsule For NEMO System

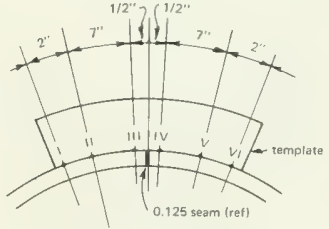

1. End of template with Roman numeral 1 is to be placed on pentagon listed first.

2. End of template with Roman numerat VI is to be placed on pentagon listed second.

3 Measure sphericity at each Roman numeral on template with a feeler gage and record

4 When taking corner measurement place template 0.50 inch from corner.

5. Templare should be perpendicular to intersection of pentagons in both directions when taking measurements.

6. Locations of points on pentagons can be found on Figure A-12

7. All readings larger than zero denote radius of curvature longer than 33.000 inches.

Typical Section Through Joint of Pentagon

Figure A-13. Quatity control measurements; deviations from nominal 33.000 -inch radius on the assembled and bonded 66-inch-diameter capsule. 


\section{Appendix B}

\section{PHOTOELASTIC INVESTIGATION OF CAPSULES}

Since acrylic plastic is a photoelastically active material, the acrylic plastic capsule lends itself to photoelastic techniques for investigation of stress distribution in the acrylic plastic parts of the hull. Photoelastic investigations may be used during fabrication for measurement of residual stresses both in the formed spherical pentagons, as well as in the bonded joints. In addition photoelastic investigations may be used for measurement of stresses during actual application of external loads to the capsule. Because the photoelastic effect functions at the molecular level, stresses can be determined quite accurately in the acrylic plastic capsule around any stress concentration, a feat that is difficult to accomplish with other stress measurement techniques.

Before the photoelastic technique, however, was applied to the acrylic plastic capsule, the photoelastic sensitivity of acrylic plastic had to be accurately determined. Since a literature search failed to uncover references to the photoelastic constants of grade G Plexiglas, the material used in the fabrication of the hull, the constants were experimentally determined as a part of the acrylic plastic capsule research program.

The photoelastic properties of the grade G Plexiglas acrylic plastic material were determined under both polarized white light and monochromatic illumination, although monochromatic illumination is generally the only one utilized for detailed experimental stress analysis. The reason for determining the photoelastic properties of acrylic plastic also under white light was that it would be hard to provide uniform monochromatic illumination for the whole 66-inch hull needed during the general overview of the stress distribution in the capsule.

The photoelastic properties of grade G Plexiglas were experimentally determined by uniaxial compression testing of a $2 \times 4 \times 1 / 2$-inch acrylic plastic test specimen. The uniaxial load was applied parallel to the 4-inch dimension, while the light was transmitted across the 0.5 -inch thickness of the material. The Polaroid polarizer and analyzer were equipped with quarterwave plates and placed parallel to the faces of the acrylic plastic specimen through which the light was transmitted. The light, after passing through the polarizer, was circularly polarized and, after passing through the stressed acrylic plastic, was analyzed by the analyzer. (The technically correct description of this photoelastic investigation technique is "determination of materials' fringe constants in a light-field polariscope utilizing circularly polarized transmitted light.") 
When a sodium vapor monochromatic light source was utilized, the successive dark fringes were observed in grade G Plexiglas material to be approximately separated by 1,600-psi principal stress or 800-psi maximum shear intervals. When placed into the fringe constant equation

$$
f=\frac{\left(\sigma_{1}-\sigma_{2}\right) \mathrm{h}}{\mathrm{n}}
$$

where $f=$ fringe constant (lb/in./fringe order)

$\sigma_{1}=$ maximum principal stress (psi)

$\sigma_{2}=$ minimum principal stress (psi)

$\mathrm{h}=$ thickness of test specimen (in.)

$\mathrm{n}=$ order of interference

the fringe constant was determined to be $f=800 \mathrm{lb} /$ in./fringe order. Since the modulus of elasticity of grade G Plexiglas is approximately 450,000 psi, the fringe constant when expressed in terms of strain is about 1,770 $\mu \mathrm{in} . /$ in./order for a 1-inch specimen thickness.

In a light-field circular polariscope utilizing monochromatic transmitted light, the first dark fringe appears in 0.5-inch-thick acrylic plastic at the 800-psi principal stress magnitude while the first light band appears at the 1,600-psi principal stress magnitude (800-psi maximum shear). That is because in a light-field circular polariscope, isochromatics (black fringes) represent half orders of interference. If the polariscope was set to produce a dark field, the dark field would be present at zero loading, while the first black isochromatic would appear only after the 1,600-psi principal stress (800-psi maximum shear) was reached.

When white light was used in the light-field polariscope utilizing circularly polarized transmission, colored isochromatics replaced the black fringes. Since it is rather difficult to convey in words the subtle changes in color that accompany the loading of acrylic plastic, color photographs were taken at many loading intervals (Figure B-1). With the aid of the colors, it is possible to determine rather readily without any elaborate experimental setup the magnitude of maximum shear stress in the completed acrylic plastic capsules. Using sunlight as the source of white light only two sheets of Polaroid with bonded quarterwave plates are needed to perform a photoelastic stress analysis of an acrylic plastic structure. 

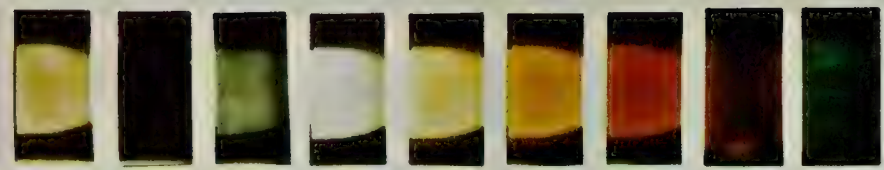

500 psi 750

1000

1250

1500

1750

2000

2250

2600

\section{COLOR STRESS CONVERSION CHART}
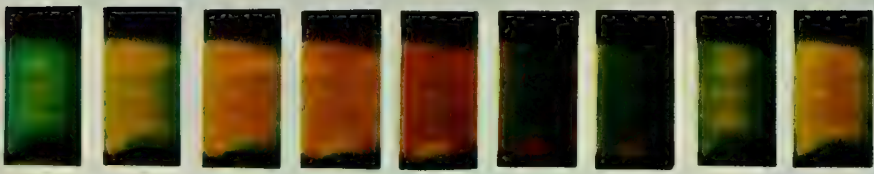

2750

3000

3250

$3500 \quad 3750$

4100

4250

4500

4750

Material: Plexiglass " $\boldsymbol{G}$ "
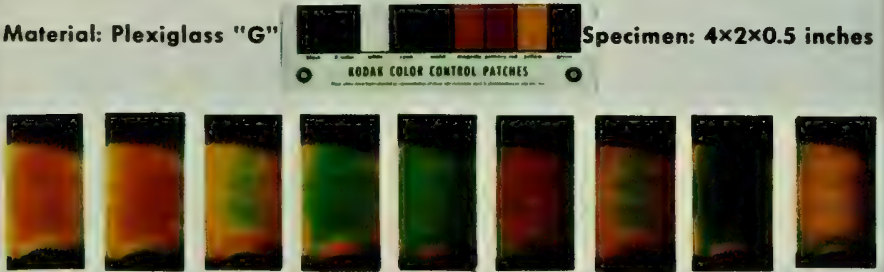

5000

5250

5500

5750

6000

6500

7000

7500

8000

Loading: Uniaxially compressed at $1000 \mathrm{psi} / \mathrm{minute}$

Lighting: Circularly polarized white light in a light field polariscope.

Figure B-1. Color stress conversion table for 0.5-inch-thick grade G Plexiglas acrylic plastic under short-term uniaxial compressive loading in a light-field polariscope utilizing a circularly polarized transmitted white light; stress values shown are for the principal stress. 
Utilizing such a simple polariscope the residual stresses were investigated in both model and large-scale acrylic plastic capsules after annealing was completed. Since the highest typical stresses in the joints are found at the corners of the pentagons, all of the measurements for typical residual stresses were performed at those locations. Using the photoelastic stress investigation technique the highest typical residual shear stresses in the acrylic plastic capsule models were found to be approximately $1,300 \mathrm{psi}$ (blue color) for solvent-bonded joints (blue, Figure 41) and 1,300 psi for cast-in-place self-polymerizing PS-18 bonded joints (blue, Figure 44). In the large-scale acrylic plastic capsule (Figure 54) the typical residual shear stresses in the cast-in-place PS-18 adhesive joint were approximately 500 psi. The magnitude of residual stresses around stress raisers in the joint is described separately, as their magnitude depends more on the quality of bonding rather than type of adhesive.

The worst residual stress raisers were found to be in the cast-in-place PS-18 joints at locations where either a trepanning operation was performed to fill a cavity or interface separation with adhesive (Figure B-2), or where one filling of the joint with adhesive terminated and another one was initiated (Figure B-3). Small or large cavities (air bubbles) in the cast-in-place adhesive joint did not act as residual stress raisers (Figure B-4).

On the basis of the photoelastic investigations into the magnitude and distribution of residual stresses in the bonded joints, several findings have been made:

1. Using the magnitude of residual stresses at the tips of the pentagons as a yardstick of comparison, there is very little difference in the effect that the solvent or self-polymerizing adhesive has on the magnitude of typical residual stresses in the joints if all the joints in the sphere are bonded in one or two increments.

2. Joints in capsules bonded in numerous small increments with self-polymerizing standard PS-18 adhesive have more stress raisers than joints in capsules bonded simultaneously by the solvent or self-polymerizing adhesive technique.

3. The stress raisers in the joints filled incrementally by self-polymerizing adhesive are the result of incremental bonding process, where one section of the joint hardens before the next one is filled with adhesive.

4. Trepanning operations (drilling a hole to a cavity in the joint and filling it with self-polymerizing adhesive) creates a serious residual stress concentration.

5. Cavities in the joint do not create significant residual stress concentrations. 


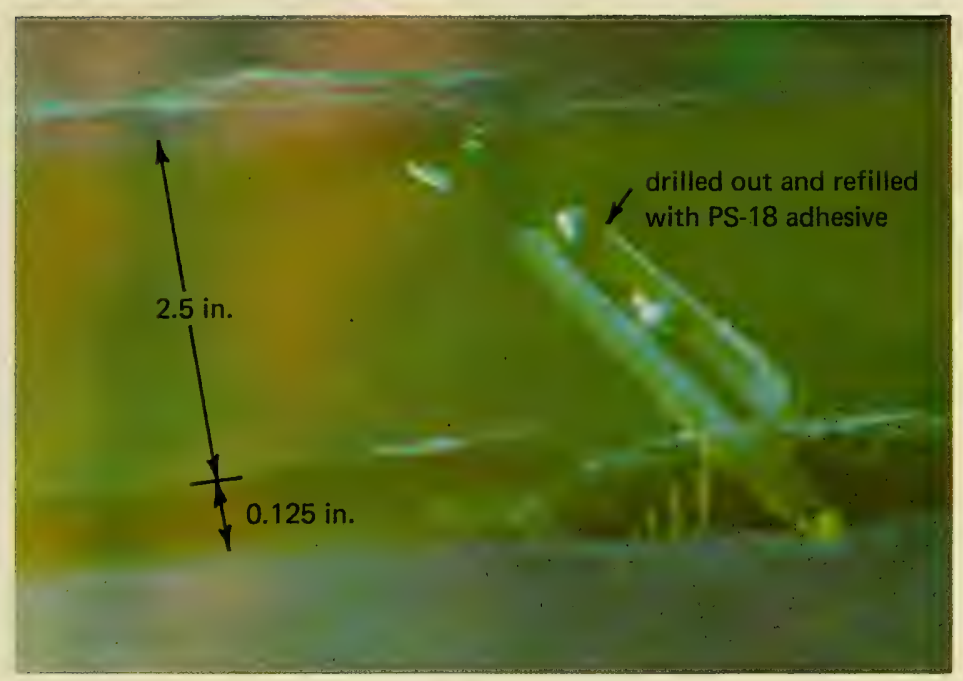

(a) Nonpolarized white light.

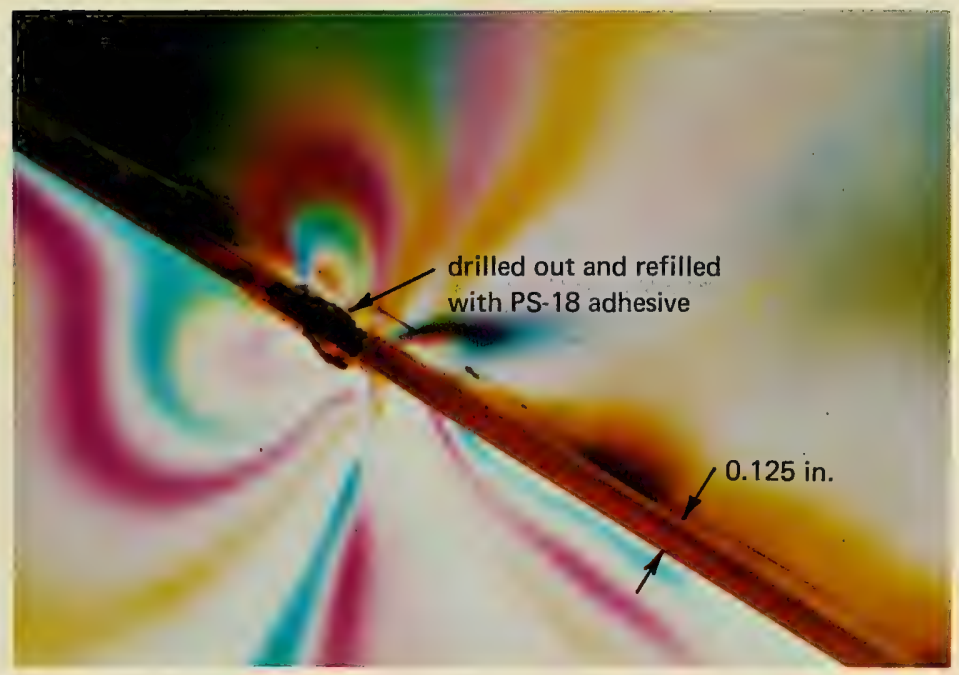

(b) Circularly polarized white light.

Figure B-2. Residual stress raiser in the form of a local trepanning and recasting operation performed on an excessively large air cavity formed at the interface between two successive pours of the PS-18 adhesive bonding the joints of the prototype 66-inch capsule. 


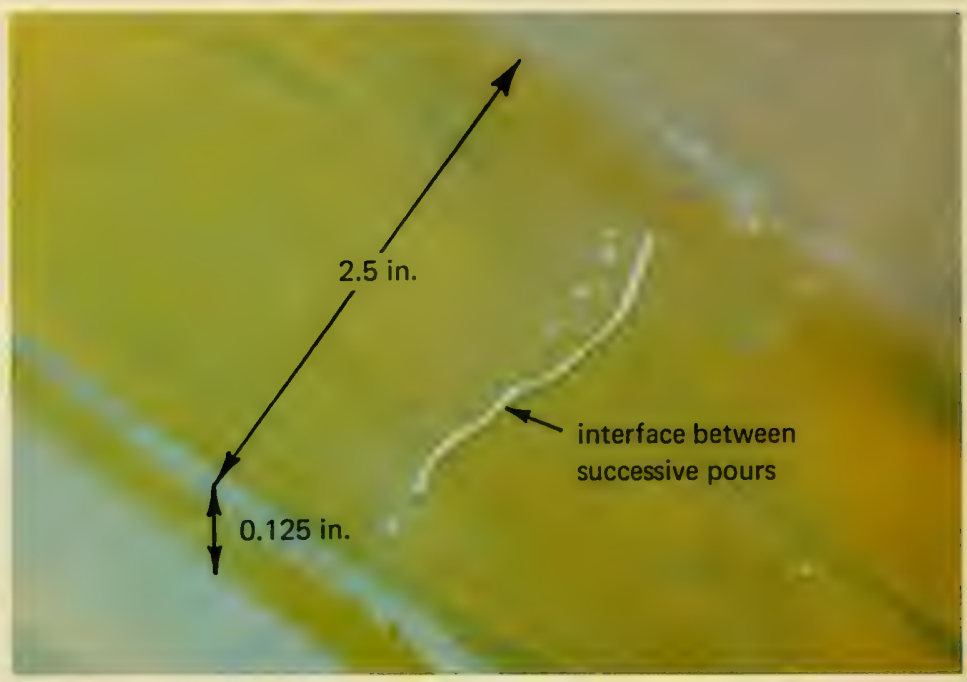

(a) Nonpolarized white light.

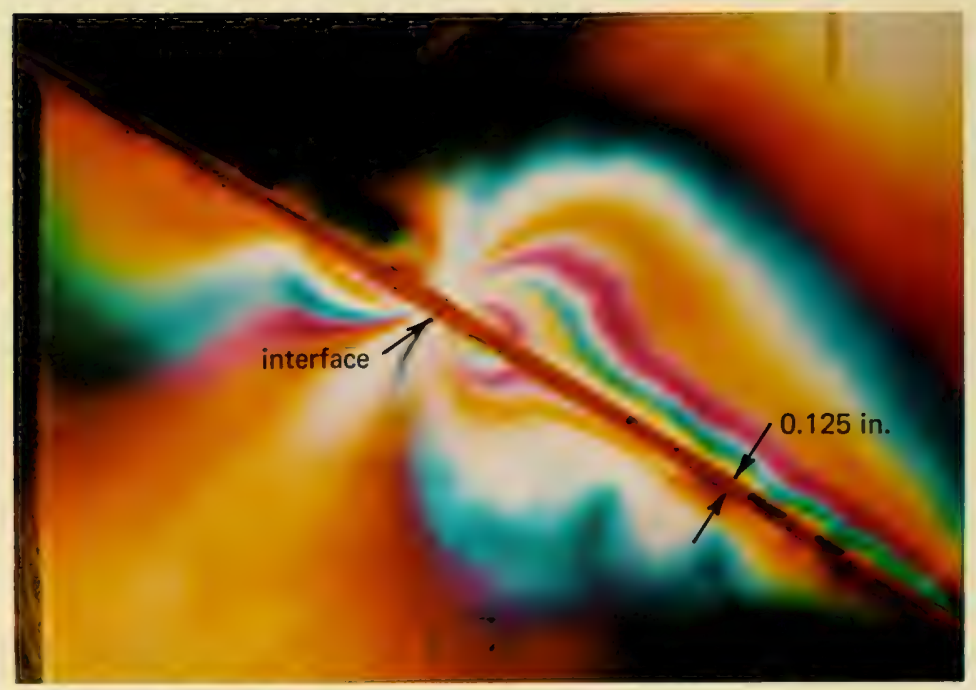

(b) Circularly polarized white light.

Figure B-3. Residual stress raiser in the form of discontinuity at the interface between two successive pours of PS-18 joint bonding material. 


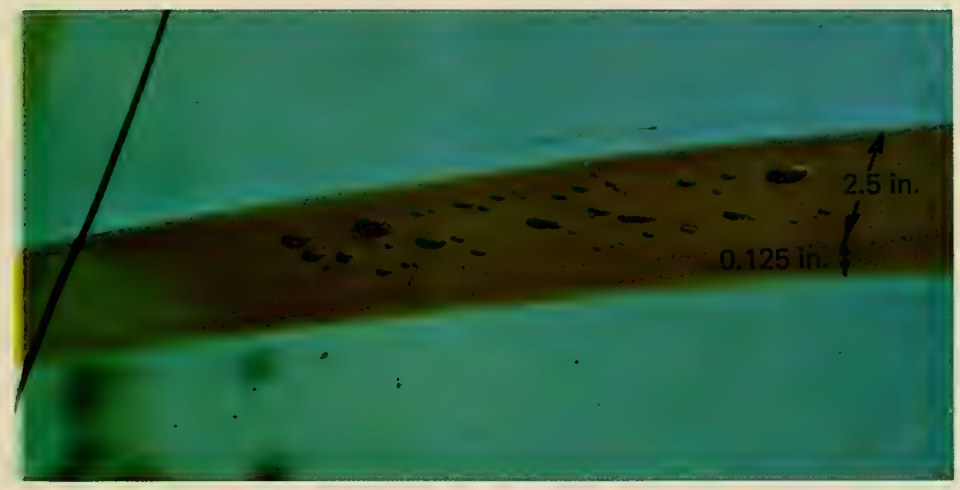

(a) Nonpolarized white light.

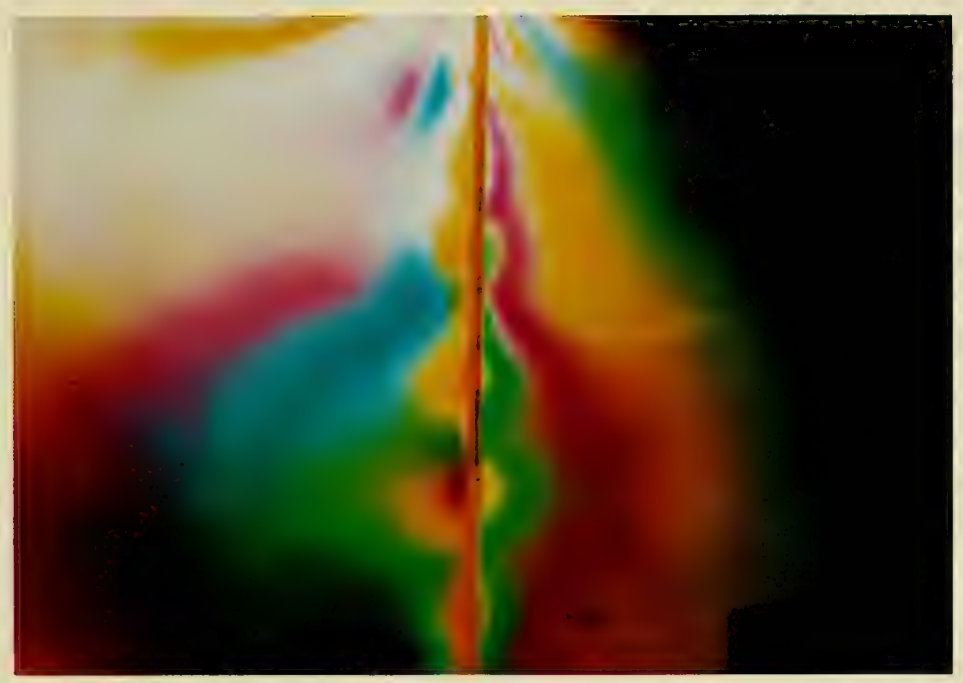

(b) Circularly polarized white light.

Figure B-4. Residual stress raisers in the form of dispersed cavities in the PS-18 joint filler. 
From these findings it appears that almost all residual stress concentrations can be eliminated from the joints if the bonding process is modified to permit complete filling of all joints in the capsule with adhesive prior to initiation of the polymerization process. In this manner polymerization of the adhesive in the joints could proceed uniformly. This will not eliminate some of the typical residual stresses in the joint; shrinking of the adhesive in the joint space, whose width is held constant by mechanical spacers, will generate tensile stresses in the filler material. These residual stresses, however, will be fairly uniform, with only a small increase in magnitude at the tips of the pentagons. Because of this uniformity the maximum typical stress will be less than if the joint was cast in increments.

Besides residual stresses, active stresses were also investigated with the photoelastic technique. The tests were performed on a small-scale acrylic plastic capsule subjected to hydrostatic loading inside an internal pressure vessel. Since it was known from other tests that the highest stresses were around the polar steel plates, only that location was investigated photoelastically. When the 15 -inch-OD $\times 14$-inch-ID acrylic plastic capsule was externally pressurized to $500 \mathrm{psi}$, the maximum shear stress value (photoelastically integrated across the wall thickness) near the edge (approximately 0.050 to 0.100 inch from the edge) of the polar penetration in the acrylic plastic was found to be approximately 1,300 psi. This experimental value compares favorably with the maximum shear stress value calculated from the extrapolated strain data measured with electric resistance strain gages at 100 psi of hydrostatic pressure near the edge of the polar penetration (Figures 82 and 83). Shear stresses could not be measured photoelastically at the very edge of the penetration because of the shadow cast by the edge of the steel hatch in the capsule.

\section{SUMMARY}

From the discussion of photoelastic investigation of residual and active stresses in the acrylic plastic capsules, it appears that this stress investigation technique is ideal for the evaluation of residual stresses introduced into the capsule by the fabrication process. In fact, the measurement of rèsidual stresses inside the joints between pentagons probably would not have been possible with any other stress investigation technique.

The photoelastic technique is also applicable to measurement of active stresses in the capsule under hydrostatic loading. However, because special equipment (pressure vessel windows and lights and a means of remotely orienting the capsule) is required for such testing, the use of electric resistance strain gages is more attractive. 
Appendix C

DIMENSIONAL DEVIATIONS OF NEMO MODELS 



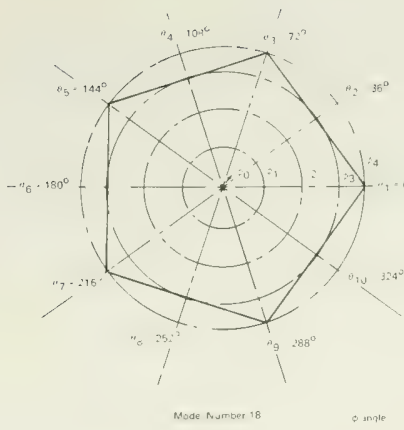

$\rho_{0}=0^{\circ} \%$

$\phi_{0}=0^{\circ} \%$
$\phi_{1}=10^{\circ} 30^{\circ}$

$\varphi^{2}-27^{\circ} \sigma^{\circ}$

31,50

Note

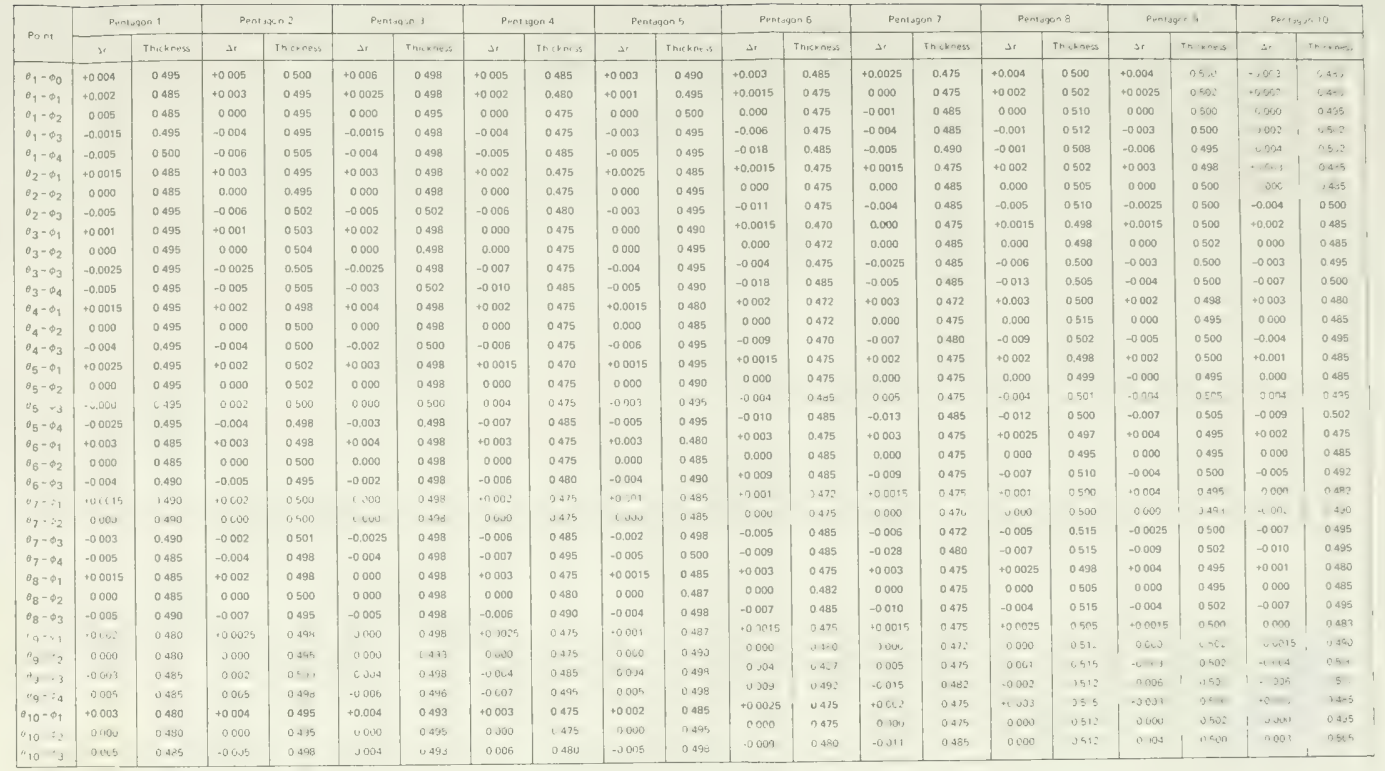




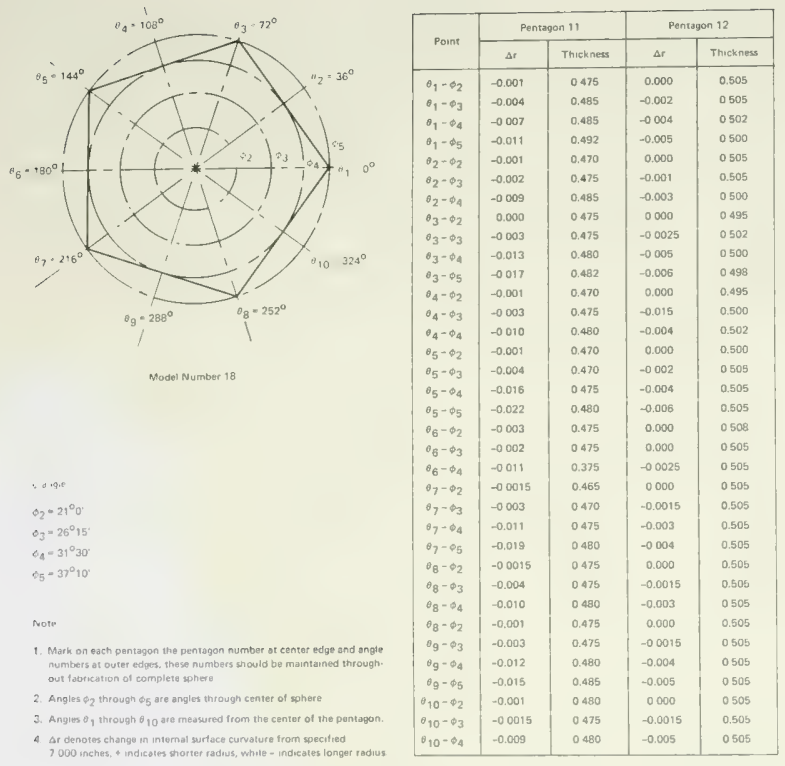

Fiture C.2. Thickness and sphiercicity measurements on machined spherical acrvhic plastic pentagons prior to assembily und bonding into a 15 -inch-diameter capsule 18 ; pentogons with polar pentetrations
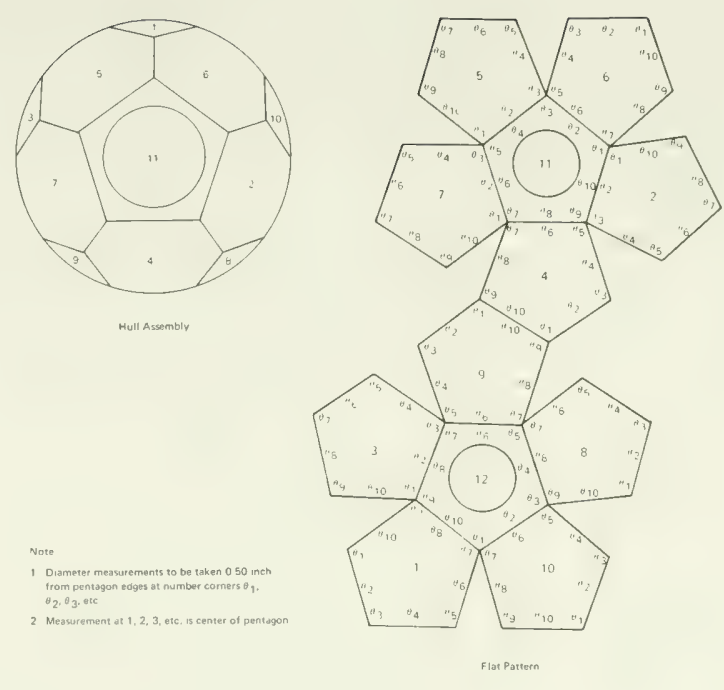

\begin{tabular}{|c|c|c|c|c|c|}
\hline From & To & Diamerere & From & To & Diametar \\
\hline$n_{1}$ & $4 \sigma_{3}$ & 15010 & $\theta_{0}$, & $9 \%$ & 14995 \\
\hline $1 w_{2}$ & $40_{2}$ & 15018 & $6 \mathrm{OO}_{2}$ & $9: 10$ & 14995 \\
\hline $1 w_{3}$ & $4 \theta_{1}$ & 15007 & $6 n_{3}$ & $9{ }^{3} 9$ & $15=0$ \\
\hline $1, \mathrm{~N}_{4}$ & 40,10 & 15023 & $\mathrm{Srog}_{4}$ & arg & 150.7 \\
\hline 1.15 & $4 " g$ & 15028 & $6=5$ & So, & 15021 \\
\hline 206 & ${ }^{4}{ }^{8}$ & 15037 & $6 \cdot 6$ & 9106 & $1 \times 6,15$ \\
\hline 107 & 4") & 15032 & 607 & ${ }^{a r_{j}}$ & 15028 \\
\hline $1+8$ & ${ }^{4 v_{6}}$ & 15040 & $6 n_{9}$ & $9.0_{4}$ & 15030 \\
\hline 1.9 & $4 n 5$ & 15026 & $6 \% 9$ & $\theta / 3$ & 15013 \\
\hline $1 \times 10$ & $4 n_{4}$ & 15037 & $6 \times 10$ & $\begin{array}{l}9 \cdot 0_{2} \\
9\end{array}$ & 15009 \\
\hline $2 " \prime 1$ & 3 & $\begin{array}{l}15038 \\
15007\end{array}$ & ,... & 10. & $\begin{array}{l}15030 \\
15028\end{array}$ \\
\hline $2 n_{2}$ & $\mathrm{HN}_{2}$ & 15013 & $w_{2}$ & $\theta$ & 15022 \\
\hline $2{ }^{2} 3$ & 301 & 15011 & $7 H_{3}$ & wi"? & 15011 \\
\hline $2 n_{4}$ & 3910 & 15032 & $m_{4}$ & 10.6 & 15011 \\
\hline $2 " 15$ & Jing & 15030 & $3 / m_{5}$ & $1+1+w_{5}$ & 10998 \\
\hline $2 N_{6}$ & ${ }^{3, n}$ & 15035 & $7 " \mathrm{p}$ & $132 \cdot 4_{4}$ & 15011 \\
\hline 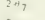 & 3."? & 15029 & $7: 7$ & $10 n_{3}$ & 15000 \\
\hline $2 n_{B}$ & $30_{6}$ & 15033 & $7 n_{\mathrm{g}}$ & an? & 15028 \\
\hline 2119 & ${ }^{2} \mathrm{n}_{5}$ & 15027 & $7 " 9$ & rant, & 15029 \\
\hline$\because 10$ & 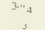 & 15030 & 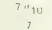 & (1), "n," & 15030 \\
\hline & $8 \mathrm{n}$ & $\begin{array}{l}150355 \\
15061\end{array}$ & $1: \theta$ & $\begin{array}{l}100 \\
120\end{array}$ & $\begin{array}{l}15033 \\
15009\end{array}$ \\
\hline "1 & $8 P_{8}$ & $\begin{array}{l}150016 \\
15017\end{array}$ & $11 \%$ & 17, & $\begin{array}{l}15008 \\
15009\end{array}$ \\
\hline $\mathrm{SO}_{3}$ & Bus & 15017 & $11 \%$ & 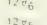 & 15009 \\
\hline 5.14 & 8,16 & 15026 & 1103 & 1205 & 14995 \\
\hline & $8.4 \mathrm{k}$ & 15024 & $11 n_{4}$ & $r_{4}$ & 15010 \\
\hline$w_{6}$ & $8 u_{4}$ & 15030 & $11 " \mathrm{~s}$ & 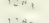 & 14995 \\
\hline $5, n$ & 80, & 15012 & $\begin{array}{l}116 \\
1.16\end{array}$ & 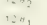 & $\begin{array}{l}15015 \\
15,113\end{array}$ \\
\hline $501 / 3$ & Q". & 15016 & $11 \mathrm{No}_{3}$ & (1:- 1, & t5. -3 \\
\hline 5.9 & $y=1$ & 15013 & 110 & $\because r_{9}$ & $15: 5$ \\
\hline$s+n, 0$ & 8010 & 15018 & 1110 & $12+n_{\mathrm{s}}$ & 15028 \\
\hline
\end{tabular}




\section{Appendix D}

\section{FEASIBILITY STUDY OF EQUATORIAL RING FOR THE ATTACHMENT OF THE ACRYLIC PLASTIC CAPSULE TO THE FRAMEWORK OF A SUBMERSIBLE SYSTEM}

\section{INTRODUCTION}

Although the external cage type of capsule restraint (Figure 8) was selected as the most applicable for securing the 66-inch capsule to the framework of the NEMO system, this does not indicate that the other types of restraint discussed in detail in the main body of the report are not applicable if the design requirements of the submersible system are different from that of the 66-inch first-generation NEMO system. Since the acrylic plastic capsules will be incorporated into submersible systems distinctly different from the first-generation NEMO system, some of the other capsule restraint concepts warranted exploratory investigation. Of the five feasible approaches previously discussed (Figures 5 through 9), only the bolt-on internal tie rod, the equatorial ring, and the external cage types of restraints appeared to show real versatility. For this reason, they were chosen for further exploratory investigation. The internal tie-rod restraint was utilized in most of the models tested for the acrylic plastic capsule program (Figures 15 and 64), and thus does not require here any further discussion. The bolt-on restraint was used during the extensive testing of the 66-inch-diameter large-scale capsule prototype (Figures 70 and 101), and for this reason no further experimental work was needed on this type of restraint. The external cage restraint was chosen for evaluation in the construction of the first generation operational NEMO system built by the Southwest Research institute, and thus the only type of capsule restraint that remained to be evaluated was the equatorial ring type.

\section{EXPERIMENTAL PROGRAM}

The exploratory experimental evaluation of the equatorial ring capsule restraint concept consisted of designing and fabricating a 15 -inch-OD $\times 14$ inch-ID acrylic plastic capsule that would be attached to the submersible's exostructure by means of an equatorial ring incorporating the essential features of that concept. The essential features of this concept (Figure D-1) were: (1) an equatorial ring (Figure D-2) to which were attached the capsule, the exostructure of the submersible, and the lifting slings; and (2) tapered pins that were rigidly seated by means of threaded nuts (Figure D-3) in the hull of the acrylic plastic capsule, while in the equatorial ring they were restrained only from lateral displacement but were permitted to translate radially by sliding in oversize holes drilled radially into the ring. 

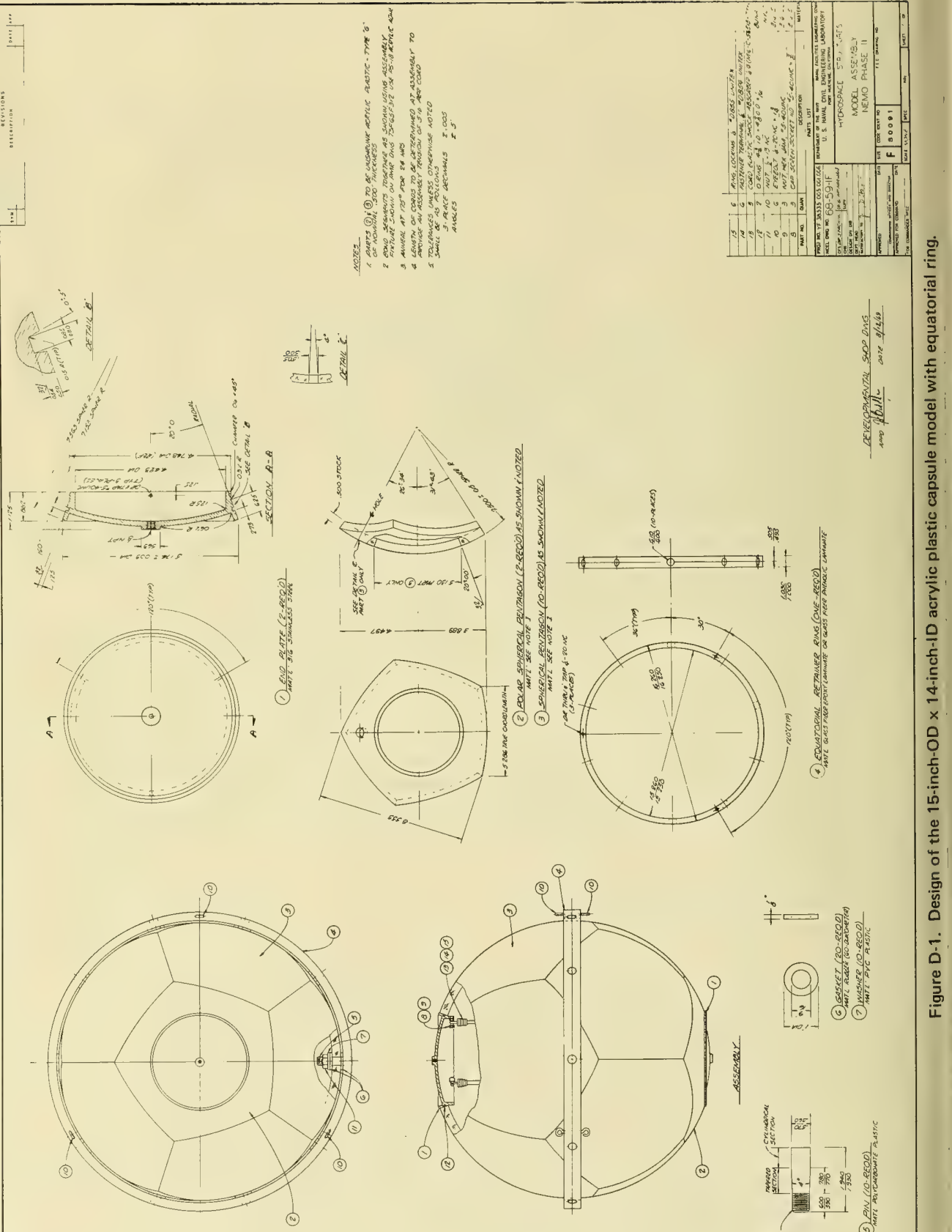


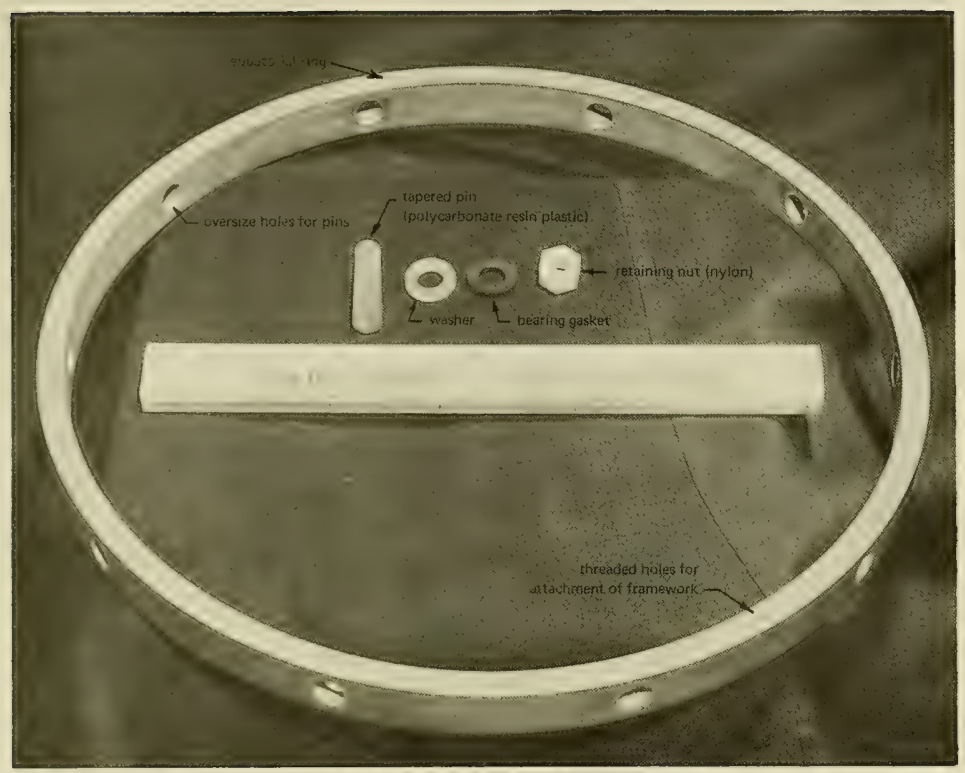

Figure D-2. Equatorial ring and tapered pins needed for attaching the ring to the acrylic plastic capsule.

The model capsule was fabricated in an identical manner to those already discussed in the main body of the report (Figure D-4), except that 10 radially tapered holes were drilled through the wall along the capsule's equator (Figure D-4). For implosion testing, the model was equipped (Figure D-5) with the simplified end closures (Figure 63) described in the main body of the report.

The test condition for imploding the equatorial ring capsule was short-term pressurization at $100^{\circ} \mathrm{F}$. The choice of this test condition was based on the program's need for implosion data on acrylic plastic capsules at various temperatures to establish the temperature-versus-implosion-pressure relationship of acrylic plastic capsules. Since the total number of model capsules available for the acrylic plastic hull program was limited, the implosion testing of the equatorial ring capsule had to satisfy two requirements. It had to establish the adequacy of the equatorial ring restraint design while at the same time it had to provide additional data for the study on the effect of temperatures on the implosion pressure of acrylic plastic capsules. 


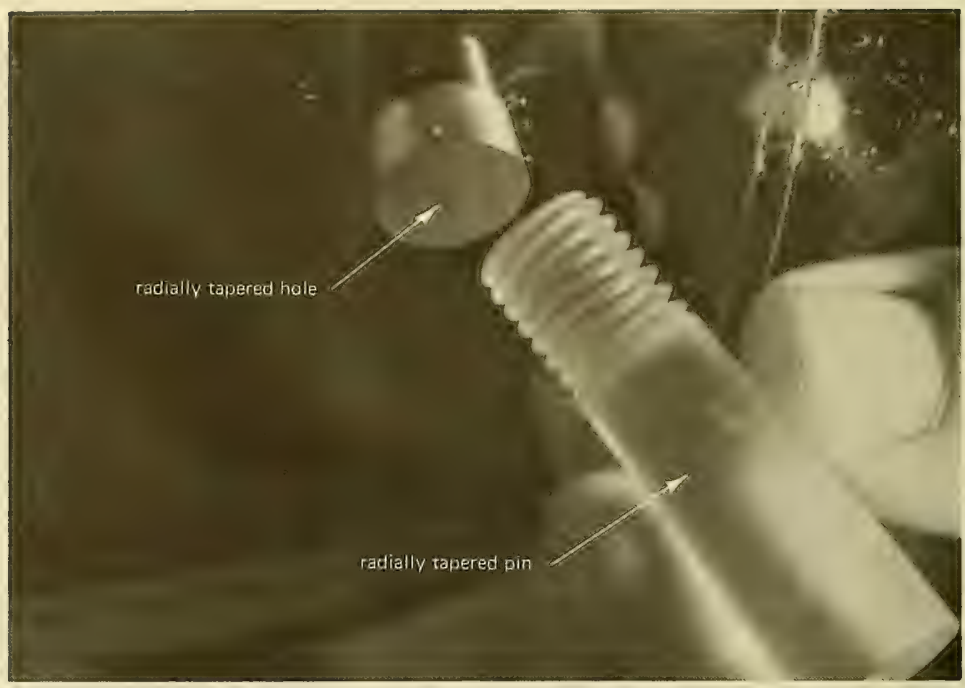

(a) Inserting the pin.

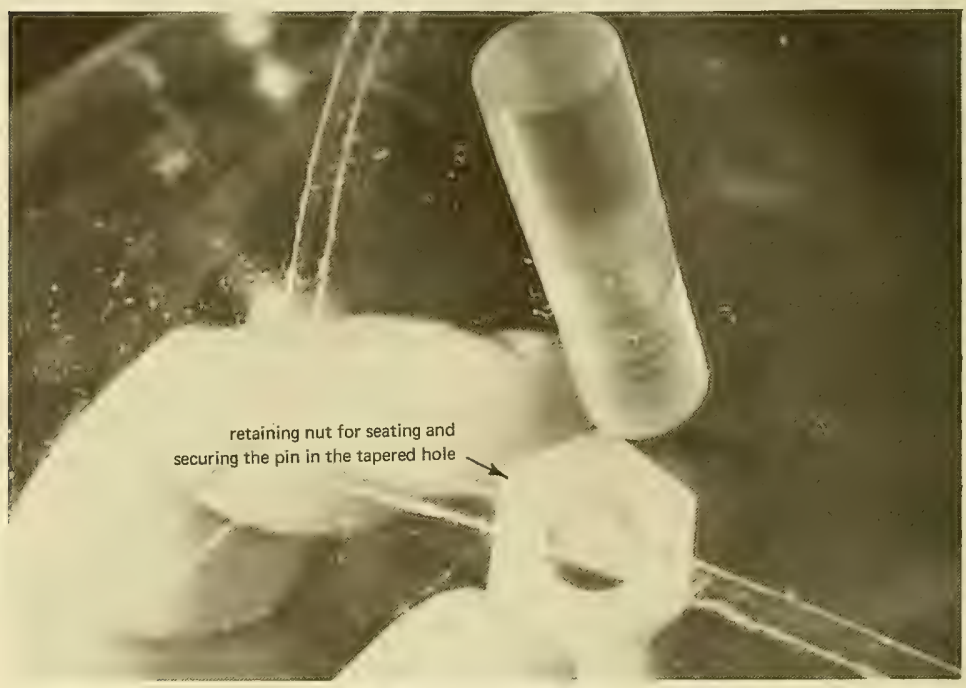

(b) Securing the pin.

Figure D-3. Installation of the tapered pins into the radially tapered holes in the acrylic plastic hull. 


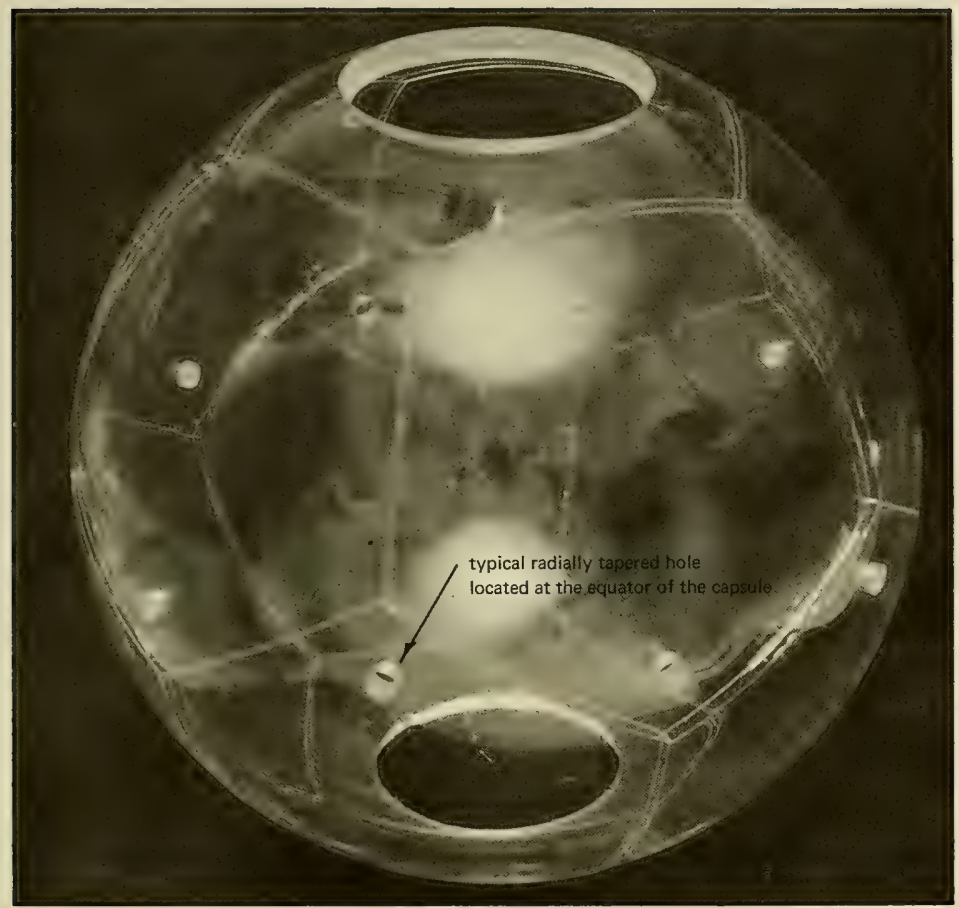

(a) Overall view of the capsule.

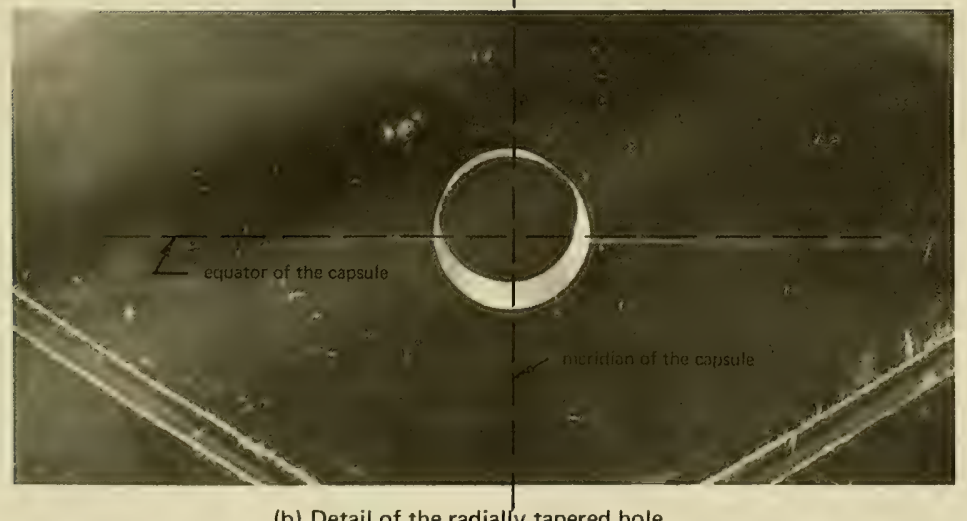

(b) Detail of the radially tapered hole.

Figure D-4. Acrylic plastic capsule prior to being outfitted with polar steel end closures, pins, and equatorial ring. 


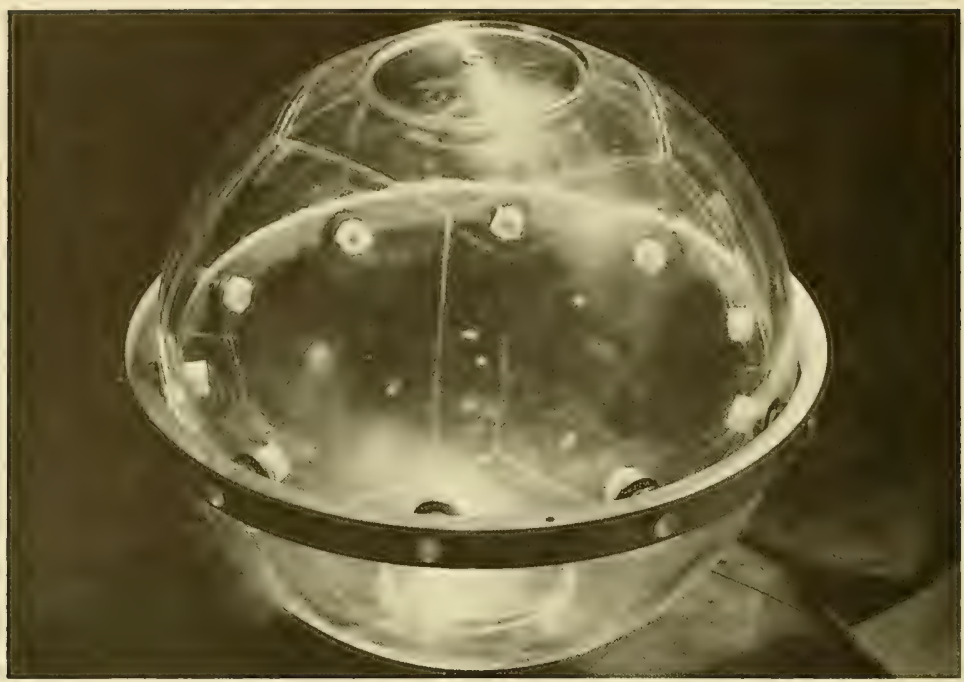

Figure D-5. Assembled 15-inch-diameter acrylic plastic capsule with equatorial ring.

After pressurization of the capsule to implosion at $100^{\circ} \mathrm{F}$, the structural adequacy of the concept was to be inferred from a plot of implosion pressure versus temperature generated by implosion testing at different temperatures on an additional eight capsules with internal tie rods. Since the critical-pressure-versus-temperature relationship appears to be linear in the 32 to $114^{\circ} \mathrm{F}$ test range, it would be easy to see whether the implosion pressure of the equatorial ring capsule fit the linear relationship established by the other capsules. A good fit would be a positive proof that the incorporation of the equatorial ring into the hull of the acrylic plastic capsule did not introduce into the hull structure any stress raisers that decrease the critical pressure of the acrylic plastic hull.

\section{FINDINGS}

When the capsule (Figure D-6) with the equatorial ring was pressurized at a $100-\mathrm{psi} / \mathrm{min}$ rate to destruction at the ambient temperature of $100^{\circ} \mathrm{F}$, it imploded at 1,175 psi. This critical pressure was found to agree (Figure D-7) with the value predicted for this temperature by the linear relationship established by the implosion pressures of other capsules at different temperatures (Figure 78). 


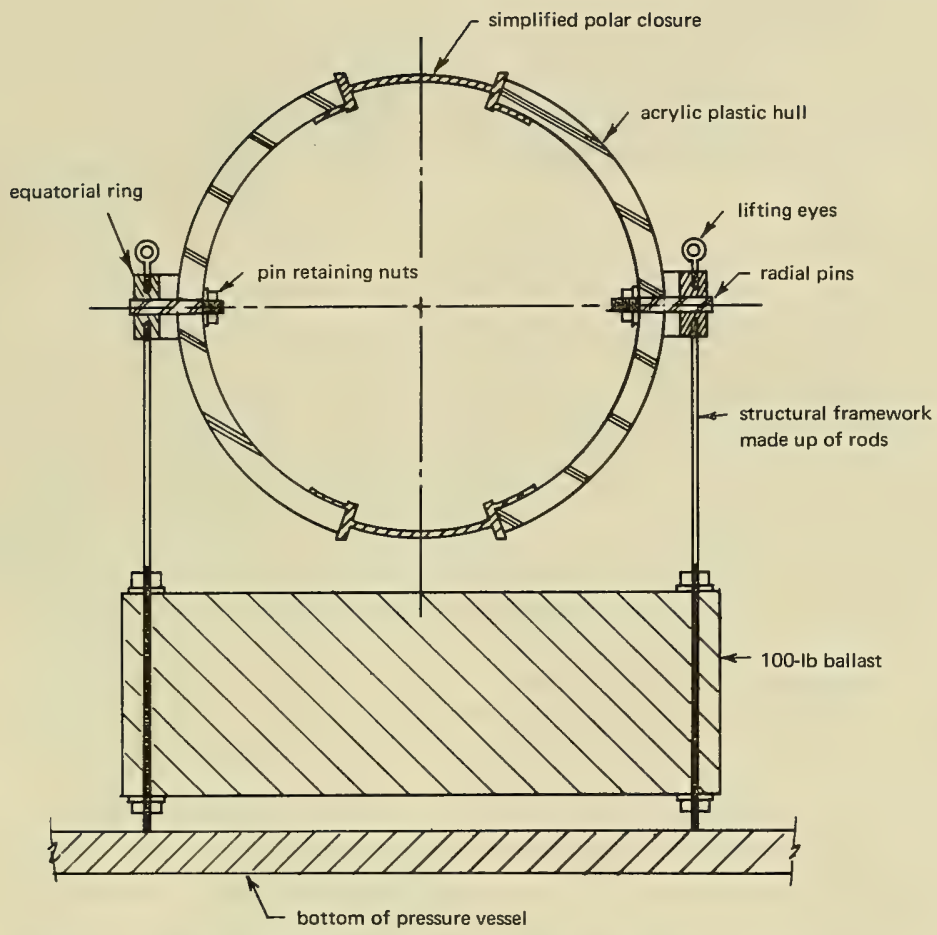

Figure D-6. Test arrangement for implosion testing of the 15-inch-diameter capsule with equatorial ring.

\section{CONCLUSION}

The equatorial ring restraint concept for attaching a spherical acrylic plastic capsule to the structural framework of a submersible system has been found to be feasible, and its application does not compromise in any manner the structural integrity of the acrylic plastic pressure hull under external hydrostatic loading. 


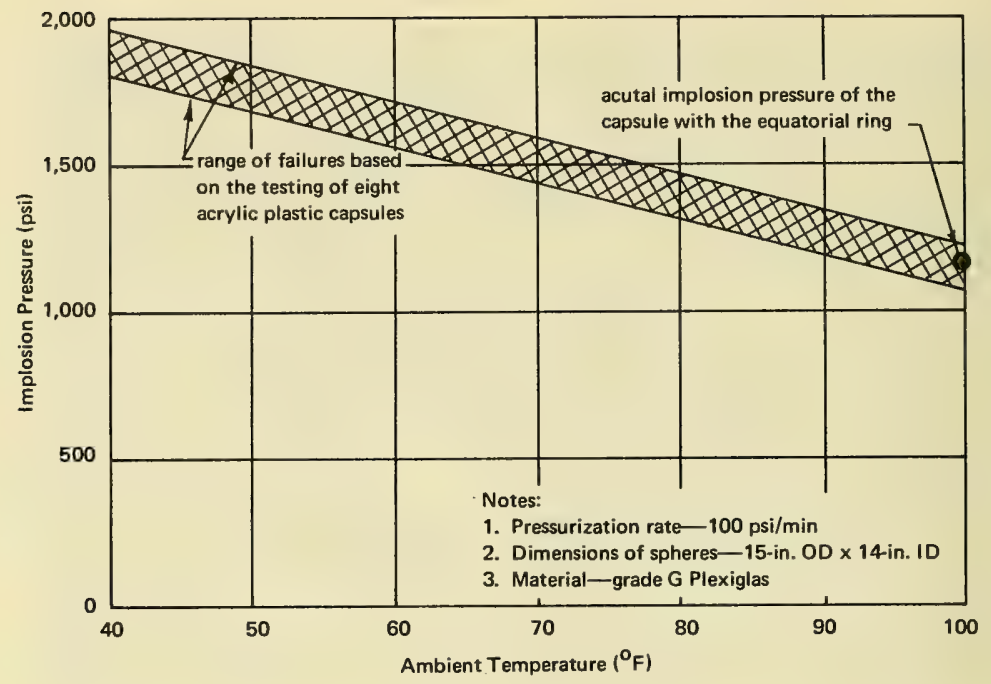

Figure D-7. Comparison of implosion pressure of equatorial ring concept with predicted implosion pressure. 


\section{Appendix $\mathrm{E}$}

\section{EXPLORATORY INVESTIGATION INTO THE FEASIBILITY OF ACRYLIC PLASTIC CAPSULES FOR OCEAN DEPTHS BEYOND 600 FEET}

\section{DISCUSSION}

The experiments constituting the main body of this report have shown conclusively that the spherical acrylic plastic capsules with nominal $t / D=0.033$ are safe for depths up to 1,000 feet and therefore merit certification for manned use to at least 600 feet. But this information does not provide the necessary basis for predicting the safe operational pressure of thicker acrylic plastic capsules. Since there may be future requirements to predict what the capsule thickness should be for some greater operational depth (for example, 2,000 feet), a brief exploratory study was performed on the collapse depth of thicker acrylic plastic capsules.

\section{EXPERIMENTAL PROGRAM}

Model capsules were used to investigate the performance of one nominal $t / D$ ratio only: 0.066 . The outside diameter and polar opening closure designs remained essentially the same as in the model capsules with $t / D=0.033$. The only differences between the simulated end closure used on the thin capsules and on the thick capsules (Figure E-1) are (1) increase in thickness of closure, (2) use of titanium instead of steel, and (3) a gasket instead of an O-ring seal system. Since only four thick capsule models were available for the exploratory study, it was decided to test them under longterm loading conditions, as in this manner a long-term failure prediction curve, similar to the one derived for thin capsules (Figure 87) could be derived. On the basis of the long-term failure curve, the safe operational depth of such a capsule could be predicted with reasonable accuracy by selecting the same span of time for definition of safe operational pressure as was found to correspond with the 600-foot operational depth for thin capsules (Figure 87). 


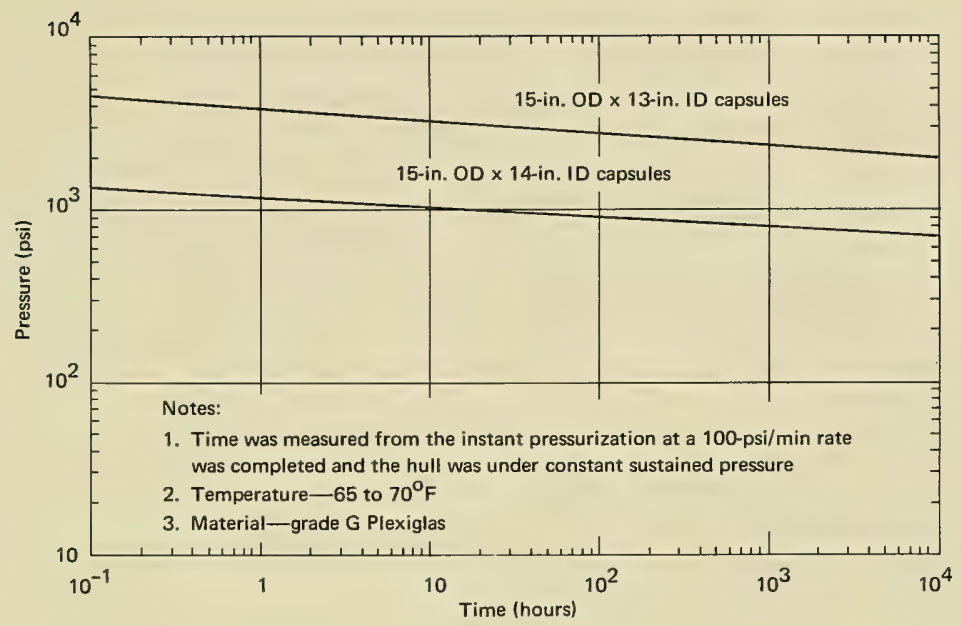

Figure E-2. Comparison of time-dependent implosion pressures for 15-inch-outsidediameter capsules with different wall thicknesses.

\section{FINDINGS}

When the four capsules with $t / D=0.066$ were tested to implosion under long-term hydrostatic loading, it was found that the implosion pressures were, as expected, a function of time (Table E-1) similar to the capsules with $t / D=0.033$. When the time-versus-implosion-pressure relationship was plotted on the same log-log scale, as was used for the $t / D=0.033$ capsules (Figure 87), it was found that the slope of the linear graph is the same as that for the thin capsule. The only difference in the results for the thick- and thin-walled capsules was the magnitude of the sustained pressure for a given duration of loading (Figure E-2). From a comparison of these results, the short-term implosion pressure of the capsules with nominal $t / D=0.066$ was extrapolated to occur at 5,000 psi, while the design proof depth (cyclic and long-term) was predicted to be 2,400 feet (certifiable for at least 1,200-foot manned operations when the same stress level and time dependent failure criteria are used as for the $\mathrm{t} / \mathrm{D}=0.033$ capsules). 


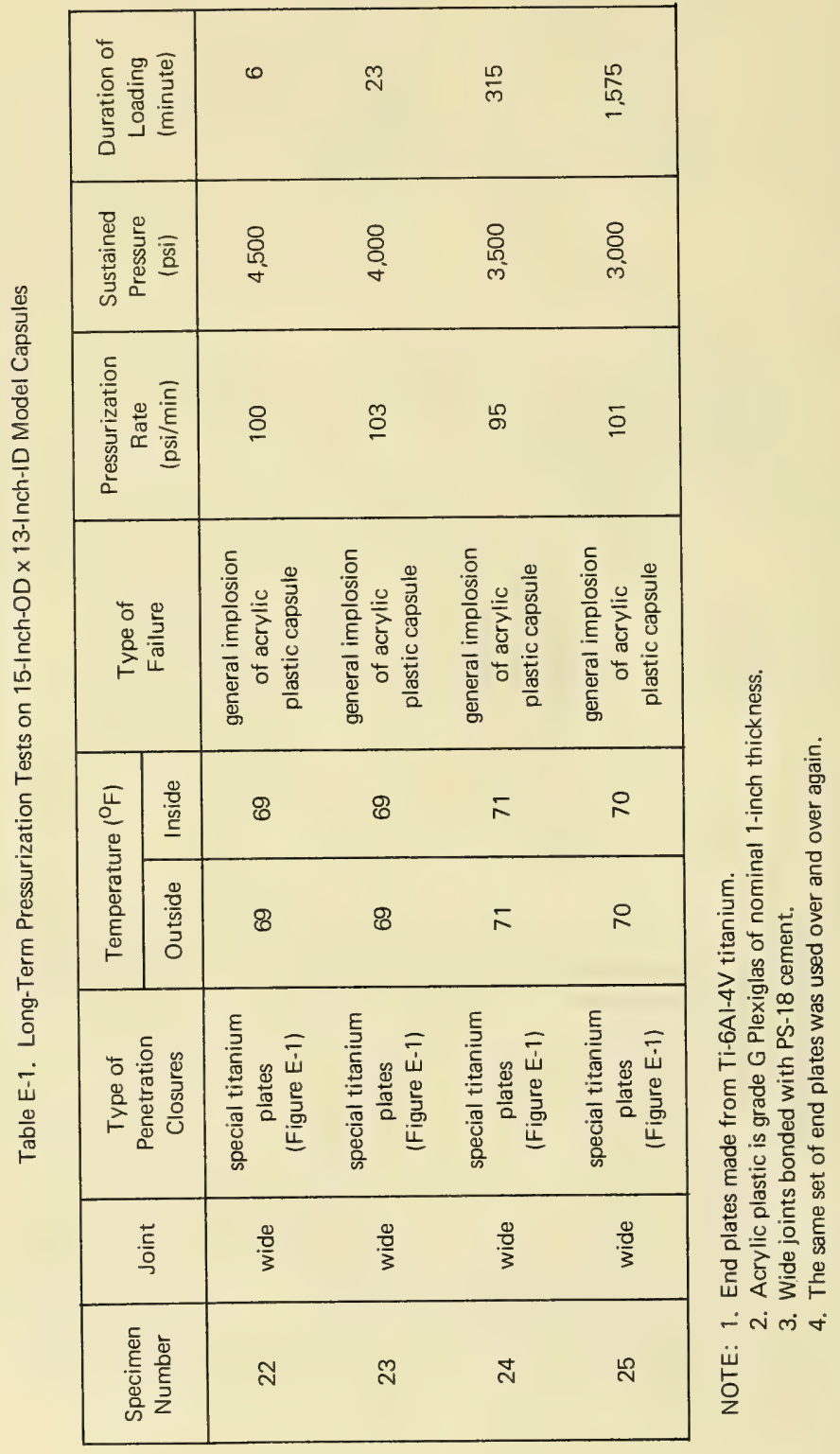


Observation of fragments from the 1 -inch-thick polar acrylic plastic pentagons confirmed the observations made previously on the 1/2-inch-thick polar pentagons that the magnitude of cracks in the beveled bearing surface pressing against the steel inserts is a function of magnitude of time-dependent strain, not stress level. Thus, for example, the cracks in the fragment (Figure E-3) from the capsule that imploded at 3,000 psi after 1,575 minutes of sustained hydrostatic loading are deeper than those found in the fragment from the capsule that imploded at 4,000 psi after 23 minutes of sustained loading (Figure E-4).

\section{CONCLUSIONS}

Acrylic plastic spherical hulls of 66-inch diameter, if fabricated from standard nominally 4 -inch-thick plate (thickest commercially available grade $\mathrm{G}$ Plexiglas plate), merit certification to an operational depth of 1,050 feet if the stress level, fatigue life, and time-dependent failure criteria are similar to those employed for certification of the nominally 2.5 -inch-thick acrylic plastic hull to a 600 -foot depth.

\section{RECOMMENDATIONS}

For operational depths either greater or lesser than 600 feet, the $t / D$ ratios shown on Figure $E-5$ are recommended for manned acrylic plastic capsules. These values of $t / D$ have been calculated using the same criteria of safety established experimentally for the NEMO 600-foot operational depth capsules of this report. When more experimental data become available on the relationship between the $t / D$ ratio and safe operational depth the Figure $E-5$ will be revised to incorporate the new findings. 


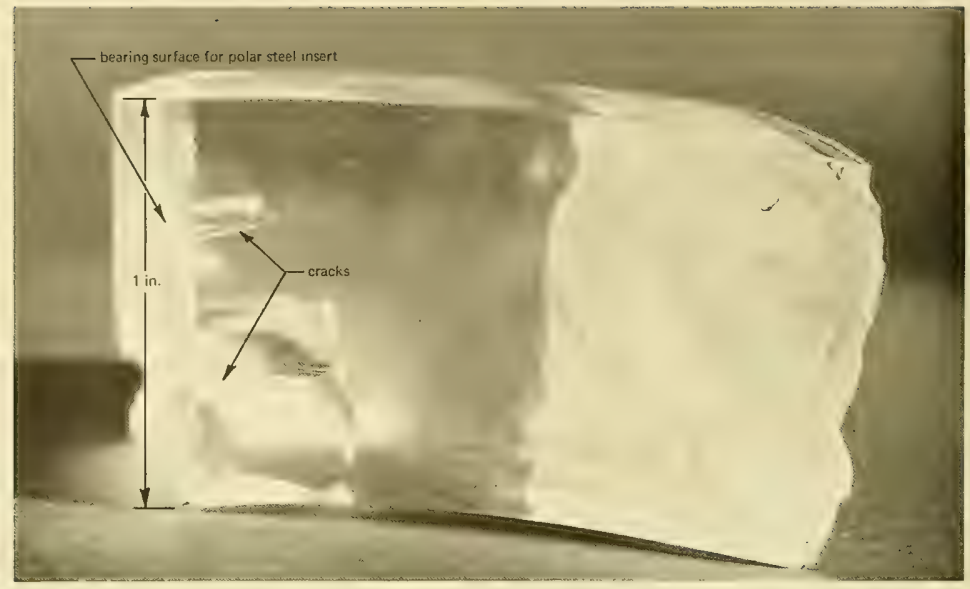

Figure E-3. Cracks in the beveled bearing surface of the polar acrylic plastic pentagon viewed at a right angle to the cut through the bearing surface. Capsule failed after 1,575 minutes of sustained loading at 3,000 psi.

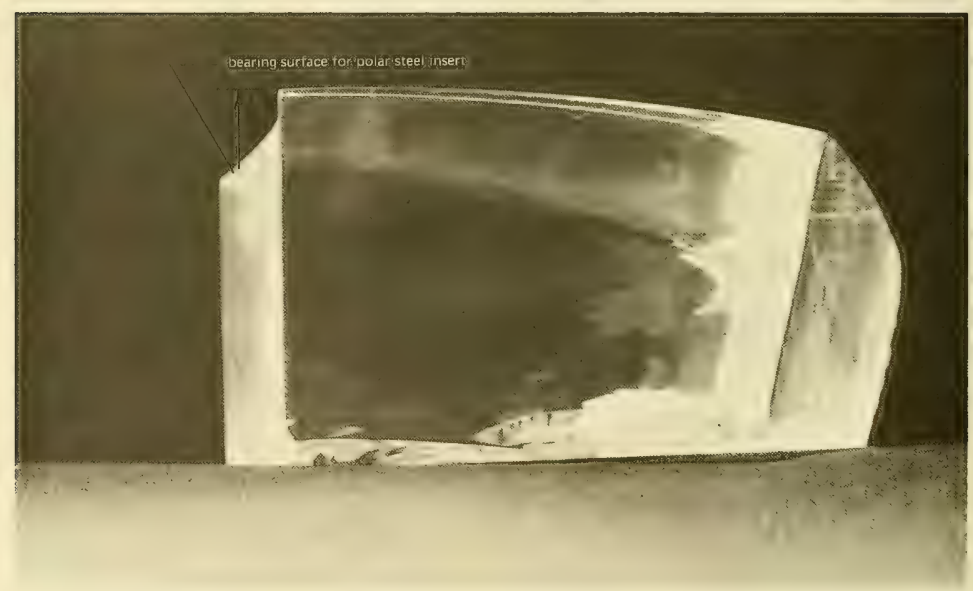

Figure E-4. Cracks in the beveled bearing surface of the polar acrylic plastic pentagon viewed at a right angle to the cut through the bearing surface. Capsule failed after 23 minutes of sustained loading at 4,000 psi. 


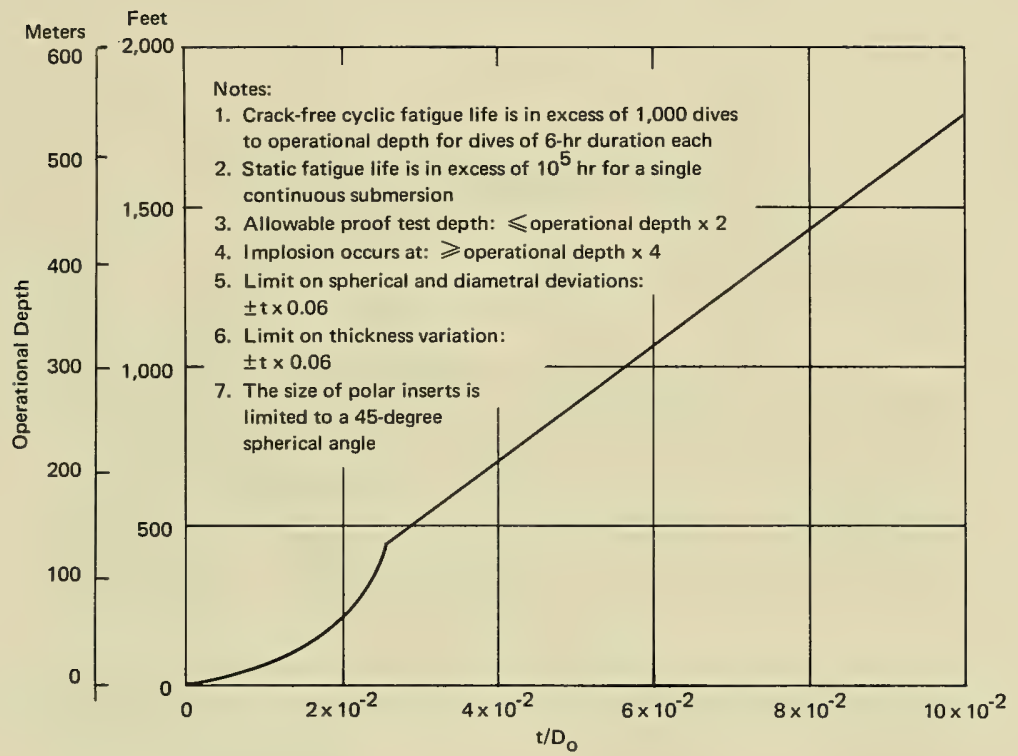

Figure E-5. Recommended dimensions of spherical acrylic capsules for certifiable manned undersea operations. 


\section{Appendix F}

\section{FABRICATION OF OPERATIONAL 66-INCH-OD $\times$ 61-INCH-ID ACRYLIC CAPSULES}

\section{INTRODUCTION}

The knowledge and experience gained in developing the prototype 66-inch acrylic capsule (NEMO capsule 0) was immediately applied to the production of three additional 66 -inch capsules. NEMO capsule 1 was to be mated with the THS (Transparent Hull Submersible) system by the Naval Underwater Research and Development Center; NEMO capsule 2 was to be incorporated into the NEMO system by the Naval Civil Engineering Laboratory; while NEMO capsule 3 was to be utilized for further structural tests.

The three additional acrylic capsules were fabricated on contract by Swedlow Inc. of Los Angeles, California. The reasons for contracting the fabrication of the additional acrylic capsules to an industrial company were: (1) to find out how well the fabrication techniques developed in a model shop environment perform in an industrial shop environment and (2) to develop in the plastics industry capability for mass production of NEMO acrylic capsules. The metal polar plates for the capsules were again manufactured by the shop personnel of the Navy Pacific Missile Range.

\section{DESIGN}

The three acrylic capsules were fabricated according to the same design used previously in the prototype 66-inch capsule. The only difference in the specification was that instead of specifying only PS-18 adhesive for the joints, other adhesives could be substituted for it providing their tensile strength was in excess of 4,000 psi and the adhesive was as immune to seawater as PS-18 adhesive.

The metal polar plates were also fabricated to the same basic design as the prototype. Major differences were only the elimination of the planetary gear hatch locking mechanism and the substitution of cadium-plated type 4130 steel for type 316 stainless steel. Both changes were made for economy. 


\section{FABRICATION}

The procedures for fabrication of capsules 1,2, and 3 were the same as for the prototype capsule 0 described in the main body of the report except that a different adhesive and acrylic plastic joint spacers were used in capsules 2 and 3. These acrylic joint spacers were not removed prior to setting of adhesive, resulting in considerable labor saving. No changes in tooling were made prior to fabricating capsules 1,2 , and 3 except for changing the curvature of the mold from nominal 33.187 to actual 33.000 inches and giving the newly machined surface a 16-rms finish.

The adhesive selected for capsules 2 and 3 was Swedlow's SS-6217, which has a polymerization rate that can be controlled more accurately during bonding of the joints. Prior to substituting SS-6217 adhesive for PS-18 adhesive, it was evaluated for strength and immunity to seawater. Both its strength and resistance to seawater (6,360-psi average tensile strength prior to immersion and 5,990 psi after 42 days' immersion) were found to be equivalent to that of PS-18. In addition, the cured adhesive was not only transparent but colorless, rather than yellow as PS-18 generally is.

\section{QUALITY CONTROL}

Very stringent quality controls were maintained during every step of the fabrication to insure that the completed capsules would perform structurally as well, or better than, the prototype capsule 0 described in the main body of the report. Five different quality controls were applied, and the results were recorded by inspectors.

\section{Material Properties}

Each sheet of $48 \times 60 \times 2.5$-inch acrylic plastic was accompanied by an invoice from the manufacturer stating the type of material, date cast, and batch number. Upon receipt of the material, a $12 \times 48 \times 2.5$-inch strip was cut from each sheet. From this strip a total of 10 (two for each test) specimens were machined and subsequently tested to determine the following material properties:

1. Compressive strength and compressive modulus of elasticity

2. Tensile strength and tensile modulus of elasticity

3. Flexural strength and flexural modulus of elasticity 


\section{Shear strength \\ 5. Compressive deformation under load}

If the average value of two test specimens for any of the above-mentioned tests was less than specified in Table 1, the acrylic sheet from which the specimens were cut was rejected. Upon examination of the test data, only one sheet out of 38 had to be rejected. The mechanical properties determined for all the 38 sheets of 2.5-inch-thick acrylic plastic are shown in Table F-1.

After the tests the accepted acrylic plastic sheets were officially released for band-sawing into 46 -inch-diameter discs.

\section{Dimensional Tolerance Measurement}

After all the discs had been cut from the accepted sheets, the thickness of each disc at seven different points was measured (Figure F-1). If the thickness of the disc was within $2.375 \leqslant t \leqslant 2.625$ inches, it was considered to be acceptable, and the given disc was released for thermoforming into sectors. None of the discs were rejected, as alt discs met the specified tolerances on thickness (Table F-2).

After forming the disc into spherical sector by means of heat and application of vacuum in a female mold, the thickness was checked again at six locations (Figure F-1). If the thickness was found to be within $2.315<t<2.685$ inches, the formed sector was considered to be acceptable, and it was released for machining into a pentagon (Table F-2).

After the pentagons were machined and annealed, they were checked for sphericity and adherence to tolerances specified for the size of the pentagon. None of the finished pentagons were found to exceed the $32.875 \leqslant R$ $\leqslant 33.125$-inch tolerance on sphericity measured at six places (Figure $F-1$ ) across the outside surface of the pentagon (Table F-2). All but five pentagons met the dimensional tolerance of $37.380 \leqslant K \leqslant 38.020$ inches on size measured between nonadjacent tips of the pentagon. The five pentagons, whose $K$ dimension was, in some cases, 0.100 -inch less than specified, were also accepted but on the condition that commensurately thicker spacers would be utilized by the contractor in the assembly of the capsule to compensate for some of the undersize pentagons. The capsule in which the undersized pentagons were used was capsule 3 not slated for manned service but structural testing. The diameter of the penetrations in the polar pentagons was checked at this time also. All of the penetrations were found to conform to the $22.568 \leqslant d \leqslant 22.578$-inch dimensional tolerance. 
Table F-1. Mechanical Properties of Acrylic Plastic Plates

(Test specimens formed from unshrunk, $48 \times 60 \times 2.5$-in. grade $\mathrm{G}$ Plexiglas.)

\begin{tabular}{|c|c|c|c|c|c|c|c|c|}
\hline \multirow{2}{*}{ Type of Test } & \multirow{2}{*}{ ASTM No. } & \multirow{2}{*}{ Specimen Size } & \multirow{2}{*}{ Test Condition } & \multirow{2}{*}{ Environment } & \multirow{2}{*}{$\begin{array}{c}\text { NCEL } \\
\text { Material } \\
\text { Specification }\end{array}$} & \multirow{2}{*}{$\begin{array}{l}\text { No. of Test } \\
\text { Specimens That } \\
\text { Failed to } \\
\text { Meet Minimum } \\
\text { Specification }^{a}\end{array}$} & \multicolumn{2}{|c|}{ Test Results } \\
\hline & & & & & & & Mimimum & Maximum \\
\hline $\begin{array}{l}\text { Tensile strength } \\
\text { (ultimate) }\end{array}$ & D-638 & $\begin{array}{l}0.250 \text { in. thick } x \\
0.500 \text { in. wide }\end{array}$ & $\begin{array}{l}0.05-\text { in. } / \mathrm{min} \\
\text { crosshead speed }\end{array}$ & $\begin{array}{l}23^{\circ} \mathrm{C} \text { and } \\
50 \% \mathrm{RH}\end{array}$ & $9,000 \mathrm{psi}$ & 1 & 8,400 psi & 10,800 psi \\
\hline $\begin{array}{l}\text { Tensile modulus } \\
\text { of elasticity } b\end{array}$ & D-638 & $\begin{array}{l}0.250 \text { in. thick } x \\
0.500 \text { in. wide }\end{array}$ & $\begin{array}{l}0.05-\mathrm{in} . / \mathrm{min} \\
\text { crosshead speed }\end{array}$ & $\begin{array}{l}23^{\circ} \mathrm{C} \text { and } \\
50 \% \mathrm{RH}\end{array}$ & 400,000 & 1 & 390,000 & 510,000 \\
\hline $\begin{array}{l}\text { Tensile elongation } \\
\text { at failure }\end{array}$ & D-638 & $\begin{array}{l}0.250 \text { in. thick } x \\
0.500 \text { in. wide }\end{array}$ & $\begin{array}{l}0.05-i n . / \mathrm{min} \\
\text { crosshead speed }\end{array}$ & $\begin{array}{l}23^{\circ} \mathrm{C} \text { and } \\
50 \% \mathrm{RH}\end{array}$ & $2-7 \%$ & 0 & $2.5 \%$ & $6.3 \%$ \\
\hline $\begin{array}{l}\text { Shear strength } \\
\text { (ultimate) }\end{array}$ & D-732-46 & $\begin{array}{l}0.250 \times 1.000-\text { in. } \\
\text { disc, punched out }\end{array}$ & $\begin{array}{l}0.05-\mathrm{in} . / \mathrm{min} \\
\text { crosshead speed }\end{array}$ & $\begin{array}{l}23^{\circ} \mathrm{C} \text { and } \\
50 \% \mathrm{RH}\end{array}$ & $8,000 \mathrm{psi}$ & 0 & $8,360 \mathrm{psi}$ & 13,000 psi \\
\hline $\begin{array}{l}\text { Compressive } \\
\text { deformation under } \\
\text { load }\end{array}$ & D-621-64 & $\begin{array}{l}0.500 \times 0.500 \times \\
0.500 \mathrm{in} .\end{array}$ & $\begin{array}{l}\text { 4,000-psi sustained } \\
\text { load for } 24 \mathrm{hr}\end{array}$ & $122^{\circ} \mathrm{F}$ & $2 \% \max$ & 1 & $0.6 \%$ & $2.5 \%$ \\
\hline $\begin{array}{l}\text { Compressive strength } \\
\text { (yield) }\end{array}$ & D.695 & $\begin{array}{l}0.500 \times 0.500 \times \\
1.500 \mathrm{in} .\end{array}$ & $\begin{array}{l}0.05-\mathrm{in} . / \mathrm{min} \\
\text { crosshead speed }\end{array}$ & $\begin{array}{l}23^{\circ} \mathrm{C} \text { and } \\
50 \% \mathrm{RH}\end{array}$ & 15,000 psi & 1 & 13,100 psi & 17,100 psi \\
\hline $\begin{array}{l}\text { Compressive modulus } \\
\text { of elasticity } b\end{array}$ & D-695 & $\begin{array}{l}0.500 \times 0.500 \times \\
1.500 \mathrm{in} .\end{array}$ & $\begin{array}{l}0.05-\mathrm{in} . / \mathrm{min} \\
\text { crosshead speed }\end{array}$ & $\begin{array}{l}23^{\circ} \mathrm{C} \text { and } \\
50 \% \mathrm{RH}\end{array}$ & 420,000 psi & 0 & 420,000 psi & $650,000 \mathrm{psi}$ \\
\hline $\begin{array}{l}\text { Flexural strength } \\
\text { (ultimate) }\end{array}$ & D-790-66 & $\begin{array}{l}0.250 \text { in. thick } x \\
0.500 \text { in. wide }\end{array}$ & $\begin{array}{l}0.11 \text {-in./min } \\
\text { deflection rate } \\
\text { on } 4.0 \text {-in. span }\end{array}$ & $\begin{array}{l}23^{\circ} \mathrm{C} \text { and } \\
50 \% \mathrm{RH}\end{array}$ & 14,000 psi & 1 & 13,200 psi & $17,200 \mathrm{psi}$ \\
\hline $\begin{array}{l}\text { Flexural modulus } \\
\text { of elasticity } b\end{array}$ & D-790-66 & $\begin{array}{l}0.250 \mathrm{in} \text {. thick } x \\
0.500 \mathrm{in} . \text { wide }\end{array}$ & $\begin{array}{l}0.11 \text {-in./min } \\
\text { deflection rate } \\
\text { on } 4.0 \text {-in. span }\end{array}$ & $\begin{array}{l}23^{\circ} \mathrm{C} \text { and } \\
50 \% \mathrm{RH}\end{array}$ & 420,000 psi & 1 & 390,000 psi & 500,000 psi \\
\hline
\end{tabular}

${ }^{a} 38$ specimens for each type of test. All of the specimens that did not meet specifications came from the same acrylic plastic plate, which was rejected as a result of it.

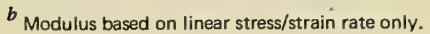





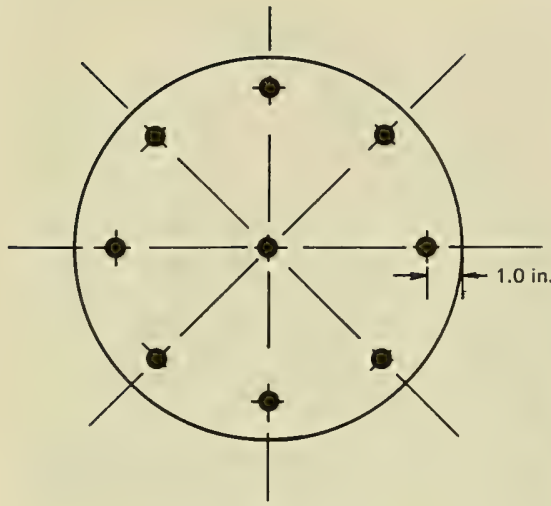

Thickness of Discs

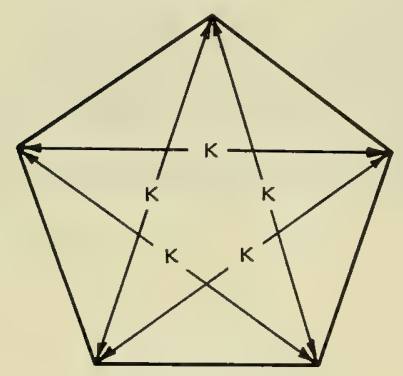

Size of Spherical Pentagons

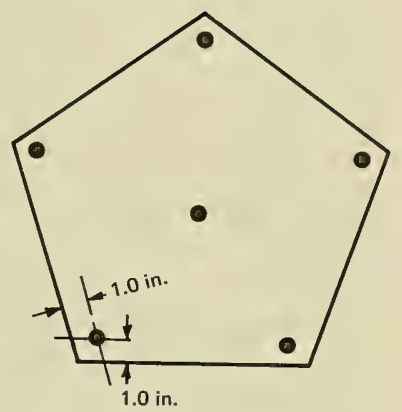

Thickness of

Spherical Pentagons

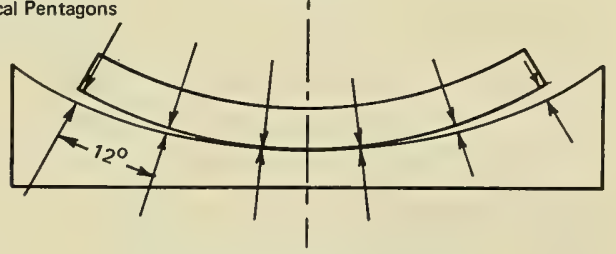

Sphericity of Spherical Pentagons

Figure F-1. Location of measurements on structural modules. 
Table F-2. Summary of Dimensional Measurements

(Measurements performed on NEMO capsules 1, 2, and 3.)

\begin{tabular}{|l|c|c|c|c|}
\hline \multirow{2}{*}{ Specimen } & \multirow{2}{*}{ Type of Measurement } & \multirow{2}{*}{$\begin{array}{c}\text { Acceptable Range } \\
\text { (in.) }\end{array}$} & \multicolumn{2}{|c|}{ Test Results (in.) } \\
\cline { 4 - 5 } & & & Maximum & Minimum \\
\hline Discs prior to forming & thickness & $2.375-2.625$ & 2.600 & 2.385 \\
Pentagons after forming & thickness & $2.315-2.685$ & 2.653 & 2.315 \\
& chord length & $37.970-38.030$ & 38.020 & 37.858 \\
& radius (sphericity) & $33.000 \pm 0.125$ & 33.100 & 32.950 \\
Completed spheres & diameter & $65.750-66.250$ & 66.100 & 66.020 \\
\hline
\end{tabular}

After assembly and bonding of the pentagons into three capsules, their diameters were checked at 16 locations and were found to be within the $65.750 \leqslant D \leqslant 66.250$-inch specified tolerance for external diameter (Table F-2). The external sphericity was so close to its nominal value that the spheres actually could have met with ease the much more desirable $66.815 \leqslant \mathrm{D} \leqslant 66.125$-inch tolerance. Since this much tighter diametral tolerance can be apparently met with ease, it is recommended for future capsules.

\section{Visual Inspection of Joints}

After all the dimensional measurements were completed, the joints (Figure F-2) were visually inspected for undercuts and presence of bubbles. No undercuts were found in any of the joints. Mismatches of thickness were found across some of the joints in the sphere's interior, as could be expected between pentagons of slightly varying thickness. Since all the variations of thickness in pentagons were previously found to be within specified thickness tolerances, the mismatches across the joints were accepted as natural.

The number of bubbles in the joints was found to be drastically fewer than in prototype capsule 0 discussed in the main body of the report and thus acceptable. Capsules 2 and 3 bonded with SS-6217 adhesive had an order of magnitude fewer bubbles than capsule 1 bonded with PS-18; capsule 1 in turn, had several orders of magnitude fewer bubbles than capsule 0, which was also bonded with PS-18 adhesive. Although the multitude of bubbles in capsule 0 did not appear to cause any reduction in the 


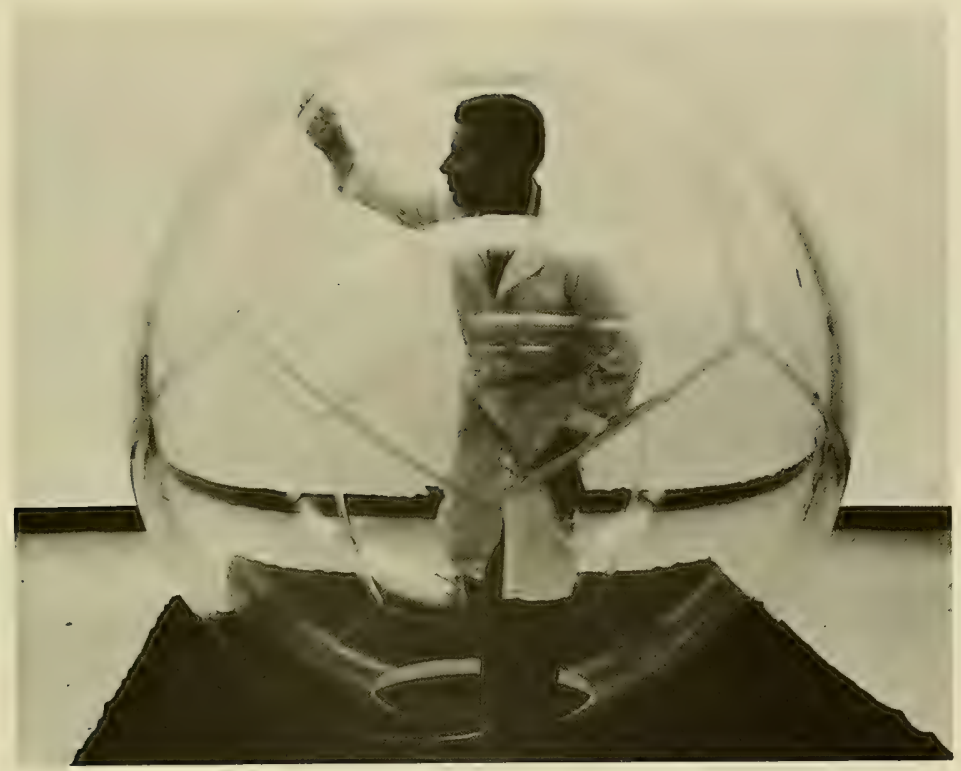

Figure F-2. Inspection of joints for presence of bubbles and undercuts at Swedlow Inc.

capsule's critical pressure or serve as sources for incipient cracks under hydrostatic loading, the substantial decrease in number of bubbles in capsules 1,2, and 3 resulted in joints with much better transparency and an improved all around appearance.

\section{Strength of Joints}

The quality of the joints was checked for each capsule by bonding together four blocks of $12 \times 4 \times 2.5$-inch acrylic plastic into two $12 \times 8.125 \times$ 2.5-inch joint specimens with the same batch of adhesive used on the capsule and in the same controlled environment where capsules were bonded. These joint specimens accompanied the capsule to the oven for annealing. After the capsule was completed and ready to be delivered to the customer, 12 tensile test coupons were cut from each joint specimen, with the joint in the center of each coupon. All of the test coupons were tested to destruction, and the ultimate tensile strength of the joints was noted. 
Table F-3. Summary of Joint Evaluation Tests

\begin{tabular}{|c|c|c|c|c|c|c|}
\hline \multirow{2}{*}{$\begin{array}{l}\text { Capsule } \\
\text { No. }\end{array}$} & \multirow{2}{*}{ Adhesive Used } & \multirow{2}{*}{$\begin{array}{c}\text { No. of } \\
\text { Test } \\
\text { Specimens } a\end{array}$} & \multirow{2}{*}{$\begin{array}{l}\text { NCEL's } \\
\text { Specified } \\
\text { Minimum } \\
\text { Tensile } \\
\text { Strength } \\
\text { (psi) }\end{array}$} & \multicolumn{3}{|c|}{ Tensile Strength (psi) $^{b}$} \\
\hline & & & & Maximum & Minimum & Average \\
\hline 1 & $\begin{array}{l}\text { PS-18 (modified } \\
\text { by Swedlow) }\end{array}$ & 16 & 4,000 & 9,300 & 7,250 & 8,580 \\
\hline 2 & $\begin{array}{l}\text { SS-6217 (developed } \\
\text { by Swedlow) }\end{array}$ & 16 & 4,000 & 7,970 & 6,340 & 7,150 \\
\hline 3 & $\begin{array}{l}\text { SS-6217 (developed } \\
\text { by Swedlow) }\end{array}$ & 12 & 4,000 & 8,640 & 6,860 & 8,300 \\
\hline
\end{tabular}

${ }^{a}$ The test specimens for each capsule were 0.750 in. thick by $0.500 \mathrm{in}$. wide. The 0.125 -in.-wide joint was located in the center of the test specimen length. Overall length of specimens was 8.125 in.

${ }^{b}$ The tensile strength of joints in prototype capsule 0 was by comparison 9,220-psi maximum, 5,680-psi minimum, and 7,350-psi average.

The tensile strength of all the joint test coupons (Table F-3) was found to be in excess of the specified minimum 4,000 psi, and thus acceptable. As can be seen from Table F-3, the tensile strength range of 6,300 to 9,800 psi of the joints in capsules 1,2, and 3 was approximately the same as for the prototype capsule (NEMO 0), for which the tensile strength of the joints was in the 5,680 to 9,200-psi range.

\section{Metallic Closures}

The quality control for the polar metal plates (Figure F-3) consisted of material control, dimensional inspection, visual finish inspection, and structural integrity testing. The material quality control consisted of notarized invoices from the supplier certifying the steel stock to be annealed type 4130 steel (MIL-S-18729C) with Brinell hardness in the 163-170 range. The dimensional control consisted of checking the dimensions of machined steel parts for conformance to the drawings. After electroplating the steel parts with cadmium without any brightener, the parts were visually inspected for appearance and quality. If the dimensional check and the visual inspection showed the parts to be satisfactory, they were accepted for mounting in the acrylic plastic capsules. 


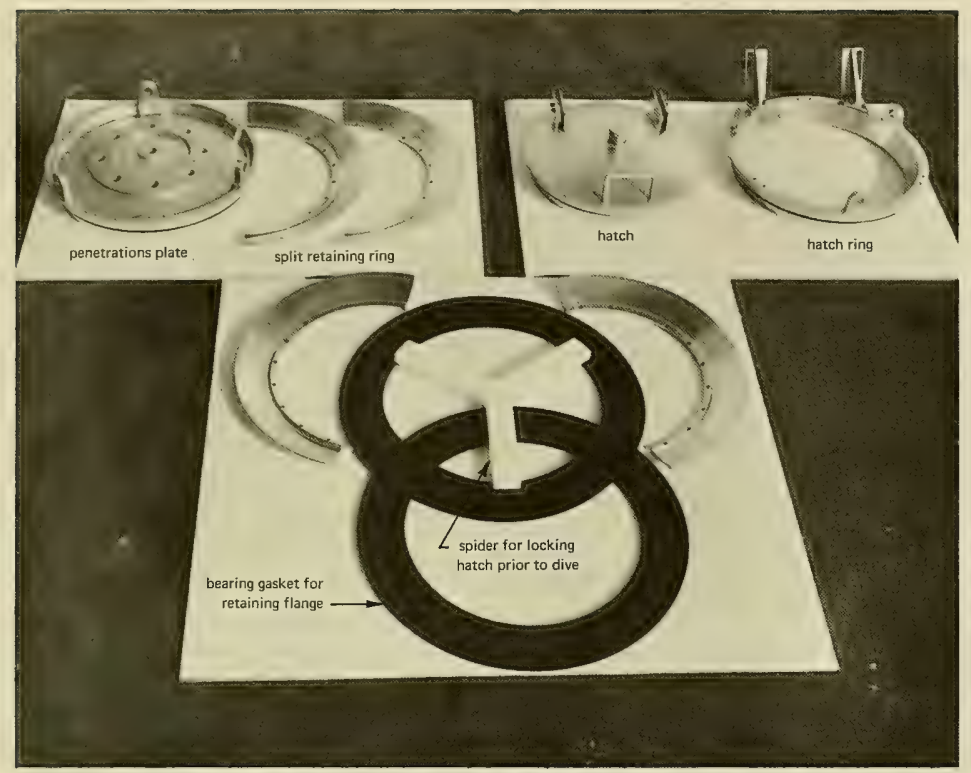

Figure F-3. Components of the top and bottom polar closures used in NEMO capsules 1,2 , and 3 .

The structural integrity test was performed on the steel parts in conjunction with the structural integrity testing of the acrylic plastic capsule, as it was felt that only by testing the steel closures mounted in the acrylic plastic capsule (Figure F-4) would it be possible to obtain a realistic evaluation for both.

\section{STRUCTURAL INTEGRITY CONTROL}

The overall structural integrity of the acrylic plastic capsules was established by a hydrostatic proof test. The acrylic plastic capsules were mated with their steel polar plates, instrumented with electric resistance strain gages, and subjected to a 4-hour hydrostatic loading under 500-psi external pressure. If no water was found in the interior of the capsule and the strains measured at the equator matched those on prototype capsule 0 under identical loading (Figure F-5), the performance of the capsule was acceptable. Since none of the three capsules leaked and the strains on the equator matched those recorded previously on capsule 0 , capsules 1,2 , and 3 
were acceptable. In addition to proof-testing capsules 1, 2, and 3, capsule 3 was subjected to extensive cyclic tests to establish the ability of SS-6217 adhesive to withstand without failure cyclic stresses and long-term immersion under compressive loading. Detailed inspection of the bonded joints failed to detect any incipient cracks after 815 cycles of 1 hour duration at 1,200-foot, 178 cycles at 1,500-foot, and 117 cycles at 2,000-foot depths. Fatigue cracks were observed in the acrylic bearing surfaces in contact with steel polar closures only after completion of cycling tests to 1,500-foot depth. The cyclic tests on NEMO capsule 3 concluded the series of quality control tests.

\section{CONCLUSIONS}

The successful production run of three 66-inch acrylic plastic capsules by a contractor according to NCEL specifications has shown that almost all of the fabrication processes developed under laboratory conditions were directly applicable with no, or only minor modifications for mass production requirements typical to industry. The only improvement was in bonding. Substitution of a different adhesive and joint spacer system permitted simultaneous bonding of six pentagons into a hemisphere rather than bonding of only a single edge at a time between adjoining pentagons.

The ability of the plastics fabricator and the material supplier to produce by industrial fabrication techniques a series of capsules with the same, or better structural properties than those found in capsule 0 , indicates that both the material properties and dimensional tolerances achieved in the prototype capsule are within the range of industrial capability. Thus, it appears that there will be no problems encountered by the industry in producing as many NEMO 66-inch acrylic plastic capsules in the future as may be required by the national undersea program.

The ability of mass-produced capsule 3 to withstand the extensive cyclic pressure testing at $100 \%$ overload without generation of fatigue cracks confirms again (1) the validity of cyclic test data generated previously by testing of models and NEMO capsule $0,(2)$ the presence of $100 \%$ overload safety margin for recommended fatigue life (Figure 121) in manned operations to 600-foot depth, and (3) the ability of NEMO capsules to withstand safely brief manned proof-test dives to 1,200 feet. 


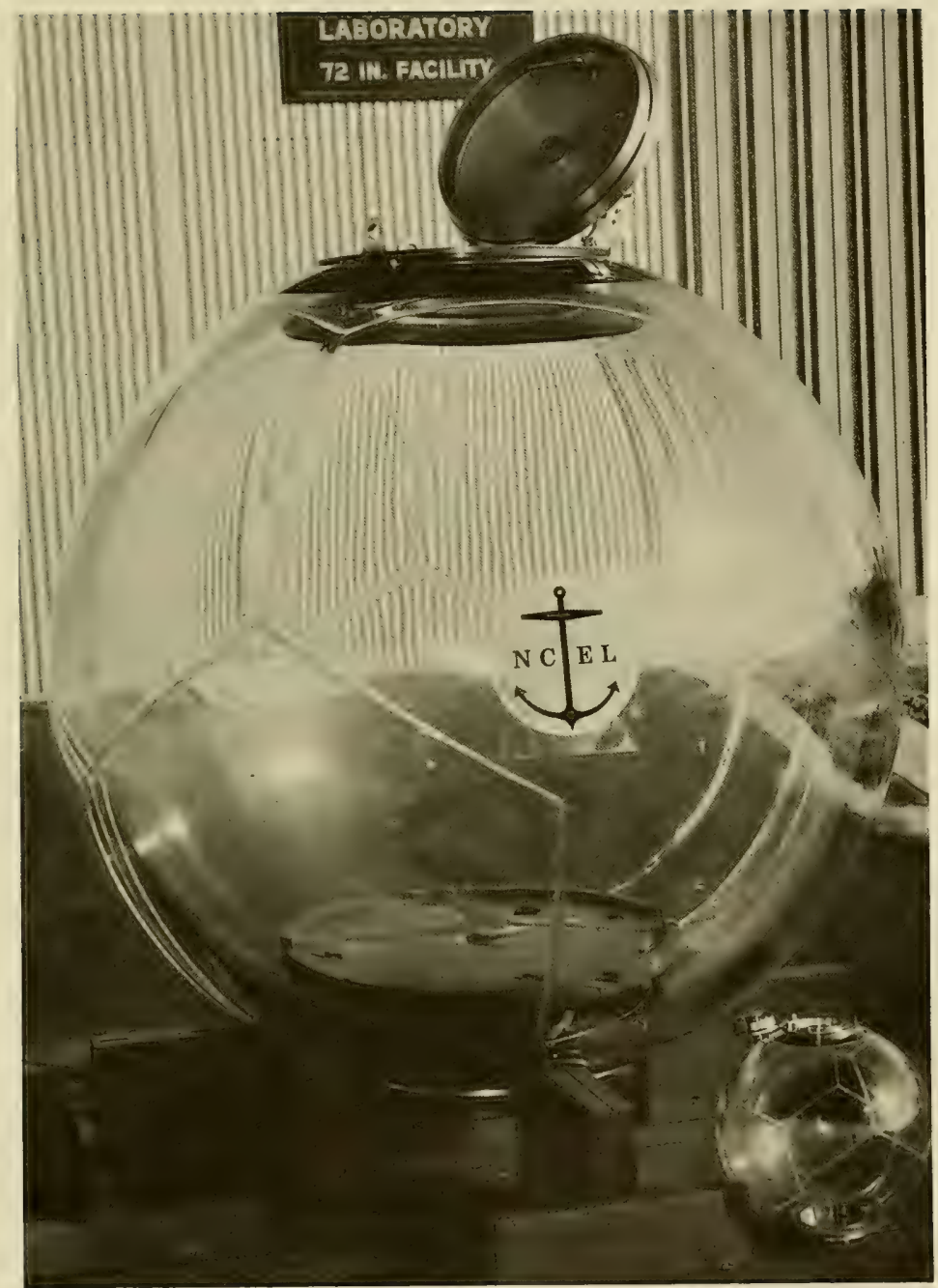

Figure F-4. Assembled NEMO capsule 1 prior to structural integrity testing in NCEL's Deep Ocean Simulation Facility; 15-inch-diameter capsule 16 is shown here for scale. 


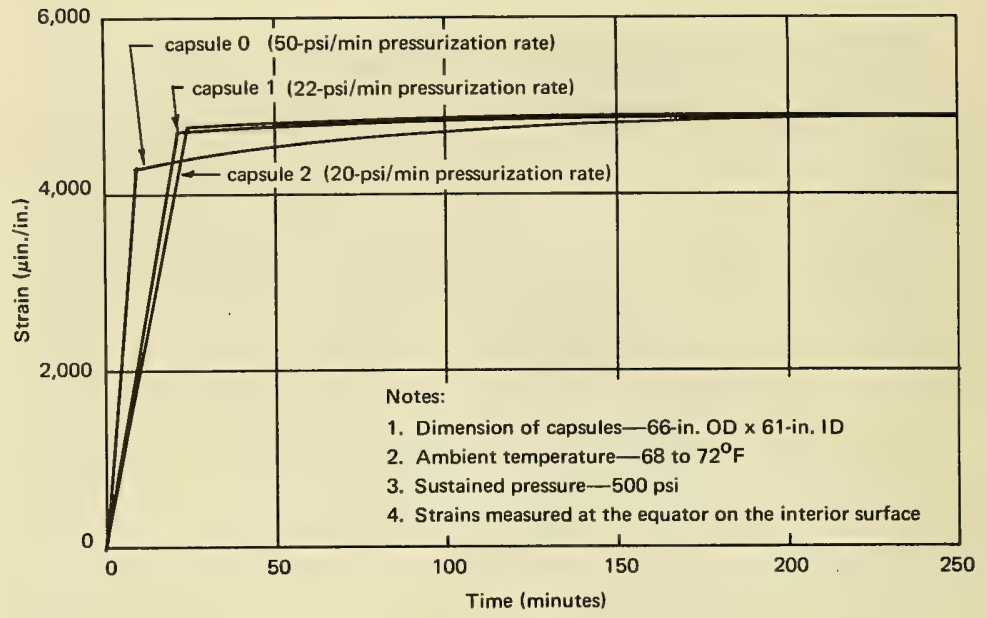

Figure F-5. Comparison of strains on the interior surface of the NEMO capsules under long term $\mathbf{5 0 0}$ psi hydrostatic loading.

\section{RECOMMENDATIONS}

The specifications and drawings used in the production of three capsules by the contractor were generally satisfactory; however, as result of the experience gained in the fabrication of the three capsules, the following specification changes should be introduced:

1. Decrease the tolerance on the diameter to $65.875 \leqslant D \leqslant 66.125$ inches.

2. Increase the tolerance on the thickness of the finished hull to $2.300 \leqslant t \leqslant 2.700$ inches.

3. Increase the tolerance on the chord length between nonadjacent pentagon tips to $32.970 \leqslant K \leqslant 33.030$ inches.

4. Increase the minimum tensile strength requirement of the joint from 4,000 psi to 5,000 psi.

5. Increase the tolerance on the thickness of the acrylic plastic discs prior to forming to $2.350 \leqslant t \leqslant 2.650$ inches. 


\section{REFERENCES}

1. A. Piccard. Earth, sky and sea. New York, Oxford University Press, 1956.

2. H. A. Perry. "The argument for glass submersibles," UnderSea Technology, vol. 5, no. 9, Sept. 1964, pp. 31-35.

3. J. G. Moldenhauer, et al. "The design, fabrication and testing of acrylic pressure hulls for manned vehicles," paper presented at American Society of Mechanical Engineers, Winter Annual meeting, Chicago, III., Nov. 7-11, 1965. (ASME paper 65-WA/Unt-10).

4. Rohm and Haas Company. Plexiglas handbook for aircraft engineers, $2 d$ ed. Philadelphia, Pa., 1952.

5. Armed Forces Supply Support Center. Military Handbook MIL-HDBK-17:

Plastics for flight vehicles, pt. 2. Transparent glazing materials. Washington,

D. C., 1961.

6. Pennsylvania State University. Ordnance Research Laboratory. Report no. NOrd 16597-91: General instability of circumferentially stiffened sandwich shells subjected to uniform external pressure, by J. D. Stachiw. University Park, Pa., Dec. 1962. (Contract NOrd 16597)

7. - Report no. NOrd 16597-97: The effects of shell joints and bonding on the stability of acrylic resin cellular shells, by J. D. Stachiw. University Park, Pa., Sept. 1964. (Contract NOrd 16597)

8. J. D. Stachiw. "The influence of joint strength and its location between cellular sandwich shell facings," Journal of Ocean Technology, vol. 2, no. 4, Oct. 1968, pp. 132-138.

9. J. D. Stachiw. "New approach to the assembly of cylindrical hulls from shell sections," Journal of Ocean Technology, vol. 2, no. 1, Dec. 1967, pp. 50-52.

10. Pennsylvania State University. Ordnance Research Laboratory. Report no. NOw 63-0209-C-2: Solid glass and ceramic external-pressure vessels, by J. D. Stachiw. University Park, Pa., Jan. 1964. (Contract NOw 63-0209-C).

11. David Taylor Model Basin. Report 1741: Tests of stiffened and unstiffened machined spherical shells under external hydrostatic pressure, by M. A. Krenzke and T. J. Kiernan. Washington, D. C., Aug. 1963. (AD 422297)

12. - Report 1757: The effect of initial imperfections on the collapse strength of deep spherical shells, by M. A. Krenzke and T. J. Kiernan. Washington, D. C., Feb. 1965. (AD 612100) 
13. - Report 1759: The elastic buckling strength of spherical glass shells, by M. A. Krenzke and R. M. Charles. Washington, D. C., Sept. 1963. (AD 423588)

14. - Report 1713: The elastic buckling strength of near-perfect deep spherical shells with ideal boundaries, by M. A. Krenzke. Washington, D. C., July 1963. (AD 416057)

15. J. W. Mavor. "Observation windows of the deep submersible ALVIN," Journal of Ocean Technology, vol. 1, no. 1, June 27, 1966, pp. 2-16.

16. Naval Civil Engineering Laboratory. Technical Report R-512: Windows for external or internal hydrostatic pressure vessels, pt. 1. Conical acrylic windows under short-term pressure application, by J. D. Stachiw and K. O. Gray. Port Hueneme, Calif., Jan. 1967. (AD 646882)

17. - Technical Report R-527: Windows for external or internal hydrostatic pressure vessels, pt. 2. Flat acrylic windows under short-term pressure application, by J. D. Stachiw, G. M. Dunn, and K. O. Gray. Port Hueneme, Calif., May 1967. (AD 652343)

18. - Technical Report R-631: Windows for external or internal hydrostatic pressure vessels, pt. 3. Critical pressure of acrylic spherical windows under short-term pressure applications, by J. D. Stachiw and F. W. Brier. Port Hueneme, Calif., June 1969. (AD 689789)

19. Naval Air Engineering Center. Aeronautical Materials Laboratory. Report no. NAEC-AML-2350: Oceanographic effects on plastic engineering materials, by H. J. Lee. Philadelphia, Pa., Jan. 1966. (AD 481419)

20. - - Report no. NAEC-AML-2260: Oceanographic effects on plastic engineering materials (Part $A$ ) and elastomeric engineering materials (Part B), by H. J. Lee and E. J. Colacicco. Philadelphia, Pa., Jan. 1966. (AD 481377)

21. Southwest Research Institute. Final report on Contract N00123-67-C2123: NEMO stress analysis and material investigation, by R. C. DeHart, T. Wah, and L. R. Calcote. San Antonio, Tex., Mar. 1968. (SwRI Project no. 03-2159)

22. Naval Civil Engineering Laboratory. Technical Note: The spherical acrylic pressure hull for hydrospace application; part II-experimental stress evaluation of prototype NEMO capsule, by J. D. Stachiw and K. Mack. Port Hueneme, Calif. (To be published.) 


\section{DISTRIBUTION LIST}

$\begin{array}{cccc}\begin{array}{c}\text { SNDL } \\ \text { Code }\end{array} & \begin{array}{c}\text { No. of } \\ \text { Activities }\end{array} & \begin{array}{c}\text { Total } \\ \text { Copies }\end{array} & \\ \text { FKAIC } & 1 & 20 & \text { Defense Documentation Center } \\ \text { FKNI } & 1 & 10 & \text { Naval Facilities Engineering Command } \\ \text { FKN5 } & 9 & 13 & \text { NAVFAC Engineering Field Divisions } \\ \text { FA25 } & 1 & 1 & \text { Public Works Centers } \\ - & 14 & 14 & \begin{array}{l}\text { RDT\&E Liaison Officers at NAVFAC } \\ \text { Engineering Field Divisions and } \\ \text { Construction Battalion Centers } \\ \text { NCEL Special Distribution List No. 9 }\end{array} \\ & 309 & 309 & \begin{array}{l}\text { Nor persons and activities interested in } \\ \text { reports on Deep Ocean Studies }\end{array}\end{array}$





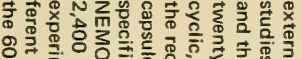

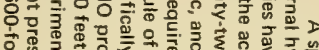

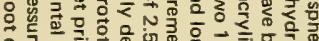

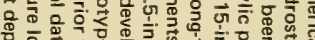

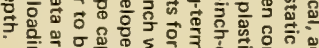

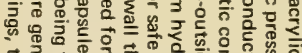

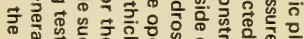

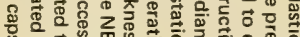

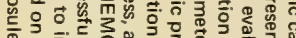

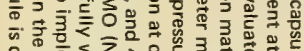

80

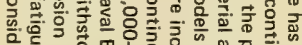

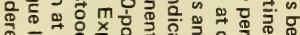

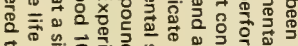

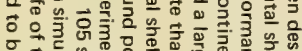

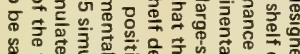

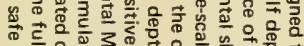

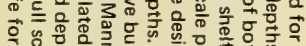

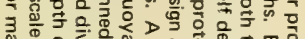

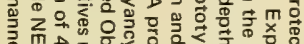

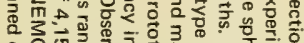

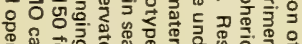

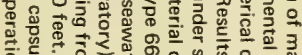

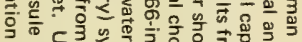

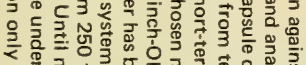

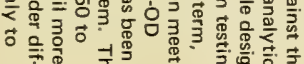

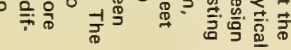

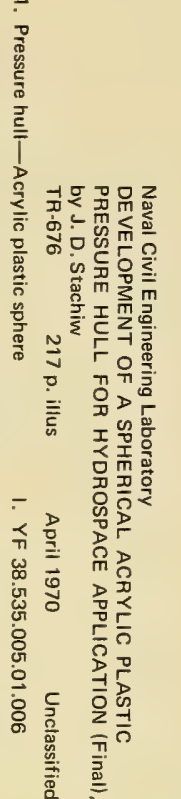

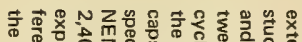

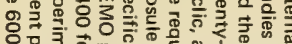

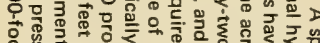

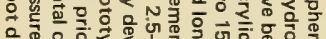

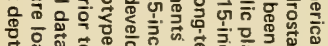

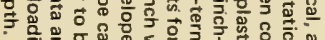

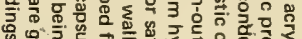

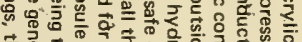

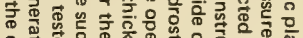

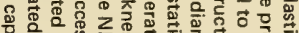

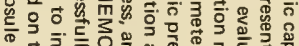

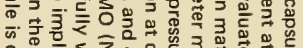

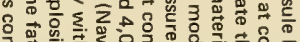

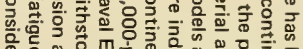

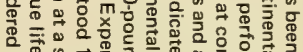

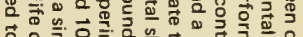

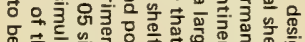

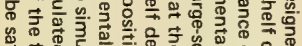

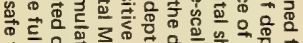
음

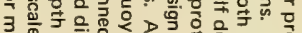

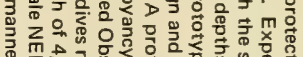

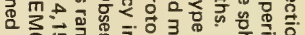

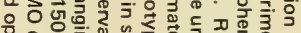

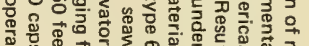

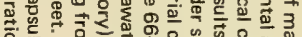

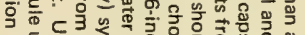

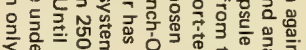
o

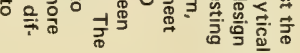

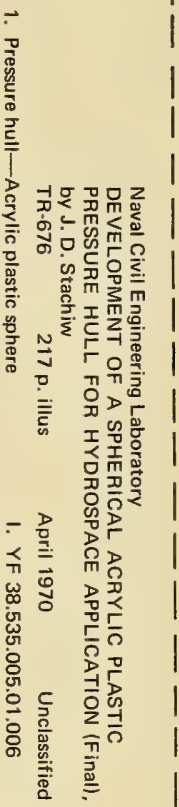

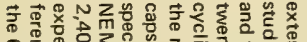

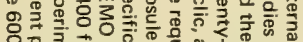

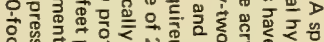

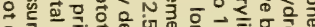
응

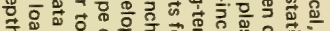

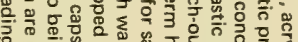

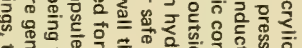

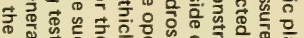

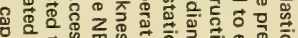

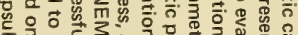

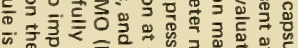

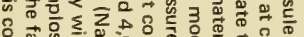

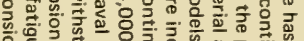

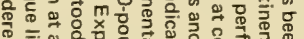

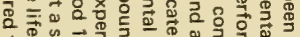

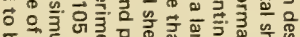

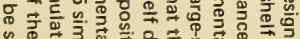

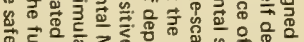

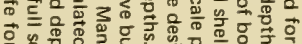

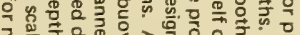

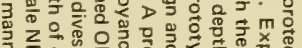

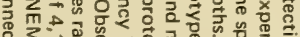

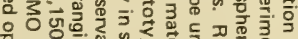

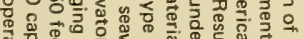

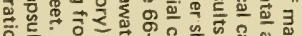

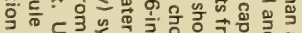

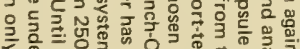

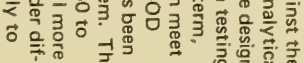

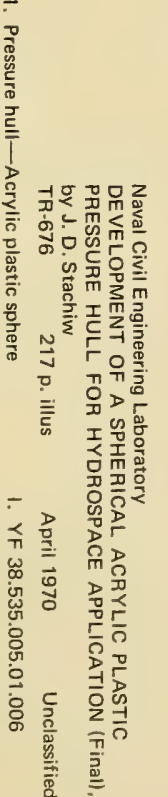

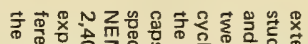

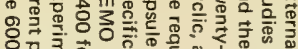

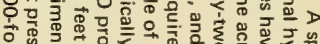

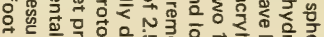
옹

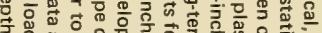

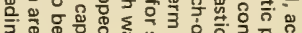

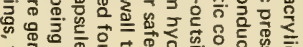

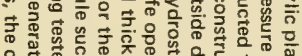

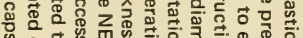

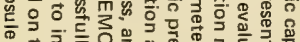

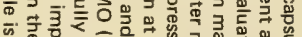
के

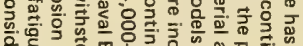
范

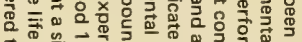

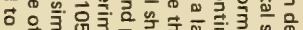

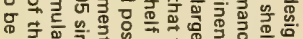

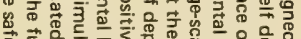

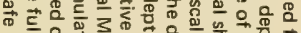
은

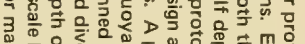

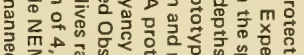

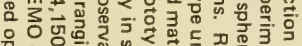

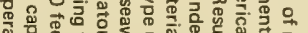

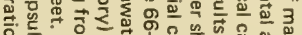

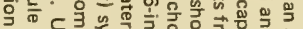

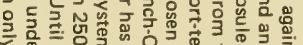

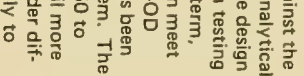

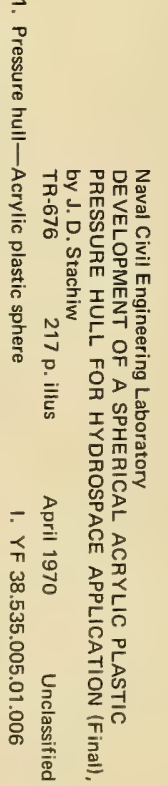





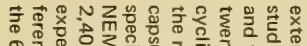

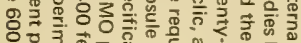

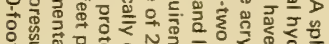

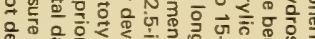

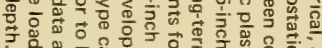

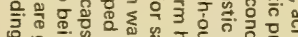

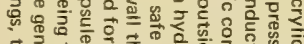

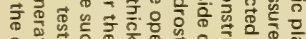

\&

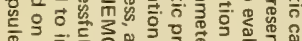

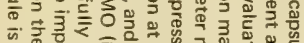

8응

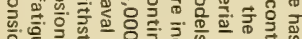

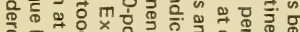

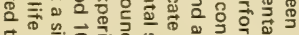

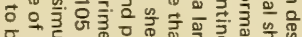

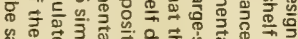

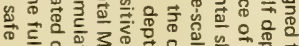
운

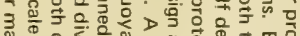

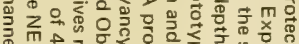

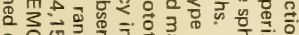

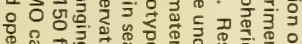
㔄。

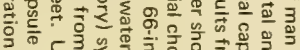

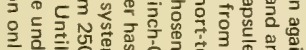

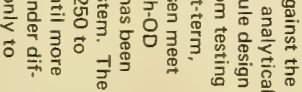

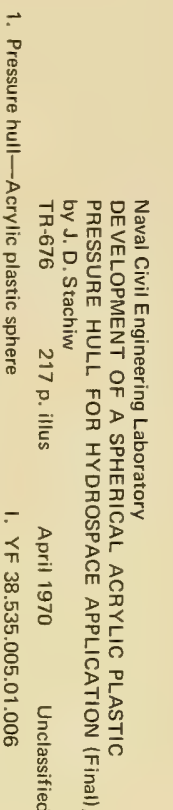

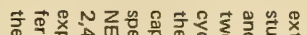

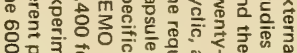

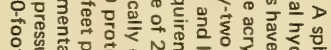

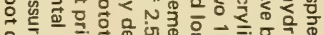

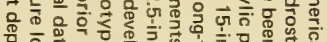

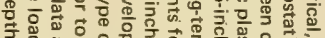

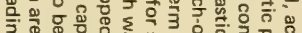

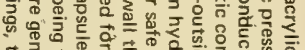

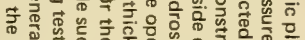

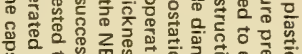

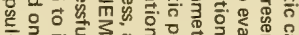

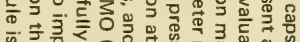

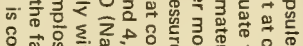

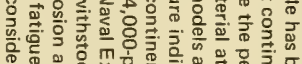

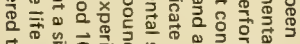
के के

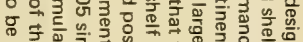
药

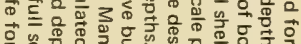

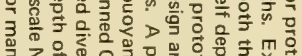

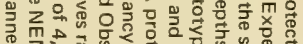

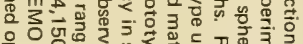

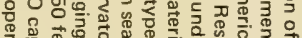

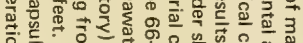

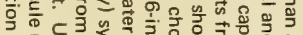

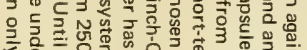

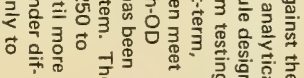

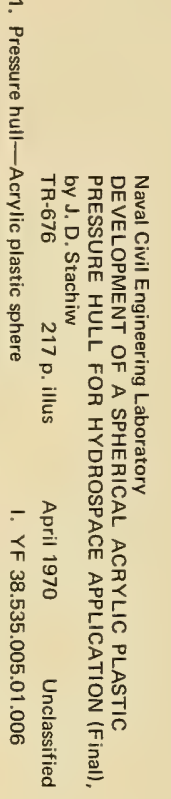

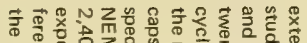

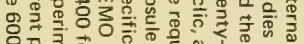

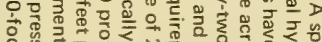

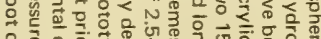

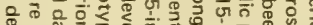

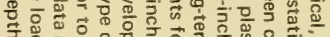

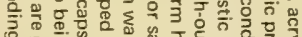

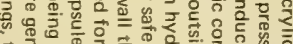

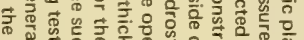

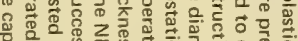

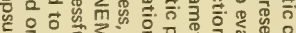

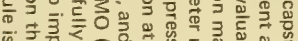

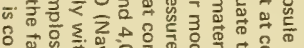

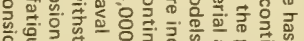

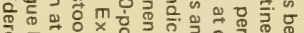
๙

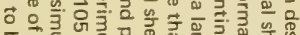

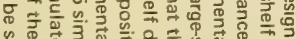

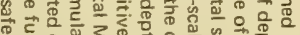

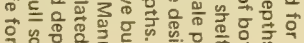

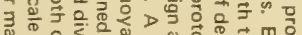

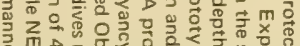

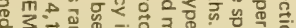
이응. पूำ

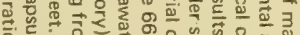

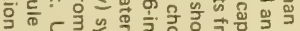

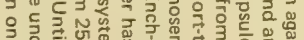

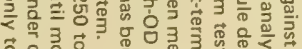

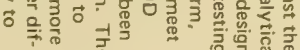

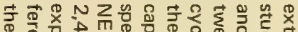

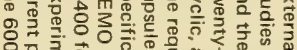

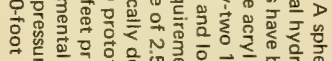
응

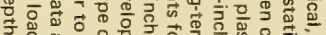

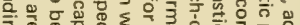

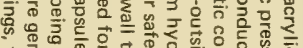

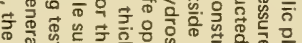

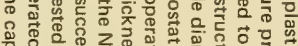

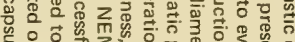

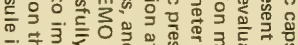

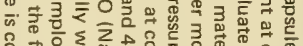

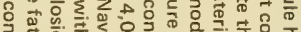

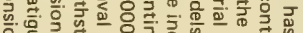

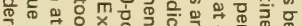

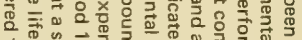
0

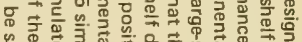

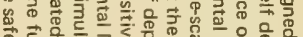

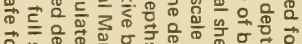

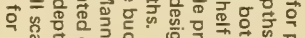

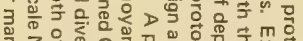

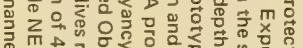

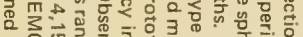
잉ํㄹ

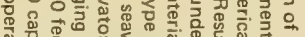

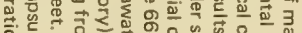

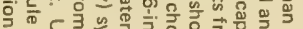

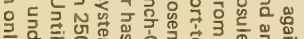

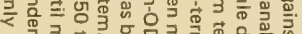

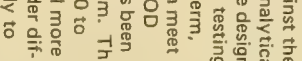

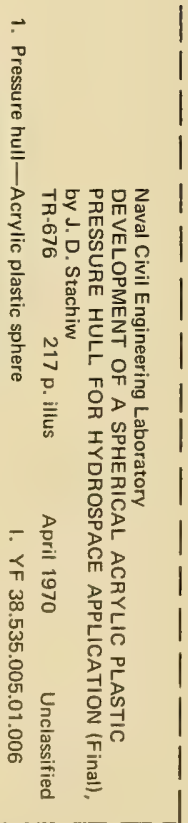



Unclassified

Security Classification

DOCUMENT CONTROL DATA - R \& D

Security classification of tille, body of abstrnct and indexing annotation nust be entered when the overall report is classified)

\begin{tabular}{|c|c|}
\hline ORIGINA TING ACTIVITY (COFporate author) & $\begin{array}{l}\text { 2a. REFORT SECURITY CLASSIFICATION } \\
\text { Unclassified }\end{array}$ \\
\hline Port Hueneme, California 93041 & $2 b$. GROUP \\
\hline
\end{tabular}

DEVELOPMENT OF A SPHERICAL ACRYLIC PLASTIC PRESSURE HULL FOR HYDROSPACE APPLICATION

\footnotetext{
4. DESCRIPTIVE NOTES (Type of repoti and Inelusive datea)
}

Final; October 1964-October 1969

5. AU THOAISI (FIrat name, middle InItiel, last name)

J. D. Stachiw

\begin{tabular}{|c|c|c|}
\hline $\begin{array}{r}\text { 6. REPORT DATE } \\
\text { April } 1970\end{array}$ & $\begin{array}{c}\text { 7a. TOTAL NO. OFPAGES } \\
217\end{array}$ & $\begin{array}{r}\text { 7b. NO. OF REFS } \\
22\end{array}$ \\
\hline $\begin{array}{l}\text { B0. CONTRACT OR GRANT NO. } \\
\text { B. PROJECTNO. YF } 38.535 .005 .006\end{array}$ & $\begin{array}{l}\text { DQ. ORIGINATOR'S REPORT } \\
\qquad \text { TF }\end{array}$ & ER(S) \\
\hline c. & $\begin{array}{l}\text { Ob. OTHEA REPOAT NO(S) } \\
\text { (his roport) }\end{array}$ & her numbers that may be assigned \\
\hline
\end{tabular}

This document has been approved for public release and sale; its distribution is unlimited.

\begin{tabular}{|l|l}
\hline 11. SUPPLEMENTARY NOTES & $\begin{array}{r}\text { 12. SPONSORING MILITARY ACTIVITY } \\
\text { Naval Facilities Engineering Command } \\
\text { Washington, D. C. }\end{array}$ \\
\hline
\end{tabular}

3. ABSTRACT

A spherical, acrylic plastic capsule has been designed for protection of man against the external hydrostatic pressure present at continental shelf depths. Experimental and analytical studies have been conducted to evaluate the performance of both the spherical capsule design and the acrylic plastic construction material at continental shelf depths. Results from testing twenty-two 15-inch-outside diameter models and a large-scale prototype under short-term, cyclic, and long-term hydrostatic pressure indicate that the design and material chosen meet the requirements for safe operation at continental shelf depths. A prototype 66-inch-OD capsule of 2.5 -inch wall thickness, and 4,000-pound positive buoyancy in seawater has been specifically developed for the NEMO (Naval Experimental Manned Observatory) system. The NEMO prototype capsule successfully withstood 105 simulated dives ranging from 250 to 2,400 feet prior to being tested to implosion at a simulated depth of 4,150 feet. Until more experimental data are generated on the fatigue life of the full scale NEMO capsule under different pressure loadings, the capsule is considered to be safe for manned operation only to the 600 -foot depth. 
Unclassified

Security Classification

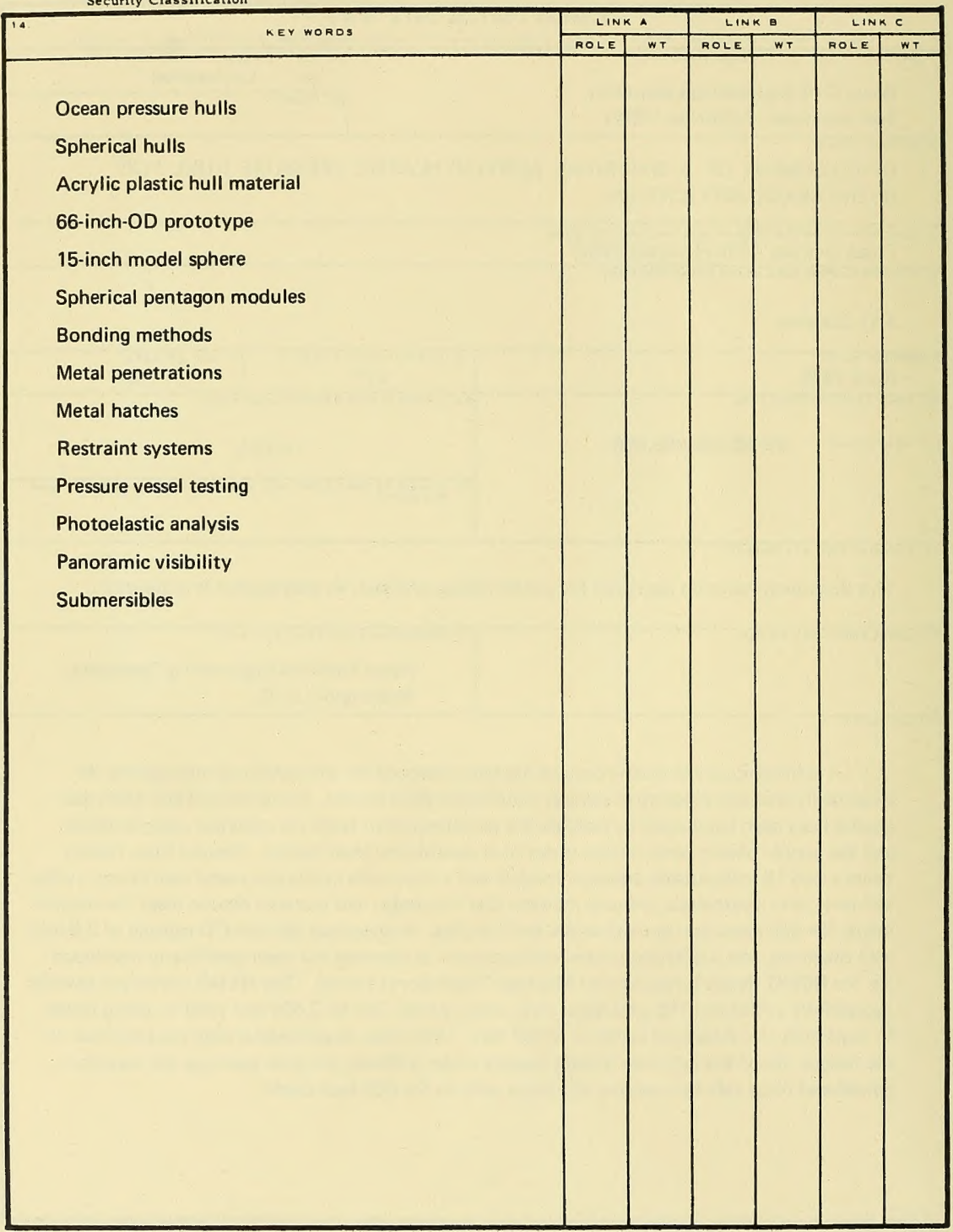


Technical Report R676 "Development of a Spherical AUTHOR ACry lic HUII for Hydron tion" April 1970

TITLE tion


r 2 